\title{
Using conservation translocations to assess the impact of anthropogenic climate change on a cold-adapted reptile, the tuatara (Sphenodon punctatus)
}

Stephanie J. Price

A thesis submitted to Victoria University of Wellington

in fulfilment of the requirement for the degree of

Doctor of Philosophy

Victoria University of Wellington

Te Whare Wānanga o te Ūpoko o te Ika a Māui

2016 



\section{Abstract}

Anthropogenic climate change is progressing at a rate unprecedented in the past 65 million years and is a significant conservation concern. The associated biotic and abiotic impacts are expected to have substantial effects on global biodiversity, with some species potentially more vulnerable than others. The tuatara (Sphenodon punctatus) is a New Zealand endemic reptile and of particular interest as it is a slowly reproducing, rangerestricted, cold-adapted ectotherm with temperature-dependent sex determination. Consequently, tuatara could be particularly vulnerable to rising air temperatures and conservation translocations have been key components of tuatara conservation efforts. Knowledge of how the tuatara might be affected by warmer climates will help inform where future conservation efforts are best directed, practices to avoid and which sites might be most suitable for the establishment of populations. The translocation of 176 adult tuatara in October 2012 from Stephens Island in New Zealand's Cook Strait to four latitudinally distant North Island sites offered the opportunity to study the responses of tuatara in a range of environments. The comparatively warmer, drier climates of several sites provided surrogates for temporal climate change, enabling an assessment of how a warming climate might impact tuatara, and how they might respond. Using field observations, laboratory analysis and controlled experiments I investigated the shortterm success of the translocations, the influence of translocation and climate on tuatara enteric bacterial communities and parasites, as well as how warmer climates might influence nocturnal activity, thermoregulatory opportunities and learning ability. I found several translocated populations to be progressing favourably, and found evidence that tuatara may exhibit enhanced growth at warmer, less densely-populated sites, suggesting that further translocations to lower latitude sites might be a viable conservation strategy. However, high population density at one translocation site was a concern and management recommendations were made to enable the dispersal of individuals. I detected Salmonella Saintpaul for the first time in a live tuatara, Campylobacter spp. was identified as a likely common commensal organism, and no measurable impact of translocation or climate on bacterial prevalence was observed, suggesting no substantial risk of climate warming to the susceptibility of tuatara to these bacteria. Tick populations were negatively impacted by translocation-associated factors following release but 
subsequently recovered at most sites and mites were not found on any translocated tuatara. Diurnal and nocturnal activities were positively influenced by air temperature, up to an upper threshold, and assessment of the site-specific thermal climates suggested that tuatara at warmer sites may benefit from increased opportunities for emergence and the attainment of preferred body temperatures throughout the year, though a higher frequency of restrictive air temperatures over summer may also reduce emergence opportunities. Experimental work showed that warmer air temperatures may enhance learning in tuatara, which could improve their ability to cope with challenging environments under climate change. However, body size was also an influential component of learning ability and further research is needed to build on these initial findings. I conclude that tuatara may experience overall benefits from further translocations to warmer sites and warming climates at currently cooler sites, which suggests that other cold-adapted reptiles with similar thermal tolerances may also see initial benefits under climate warming, though further monitoring is required to determine longer-term translocation success. Equally, while warmer air temperatures were not found to be detrimental to tuatara, they still pose a risk to population viability and further work is required on the impacts of associated abiotic factors like drought, and how populations of this long-lived species may be affected if and when climate warming exceeds the upper temperature rise of $\sim 5^{\circ} \mathrm{C}$ predicted by the $2100 \mathrm{~s}$. 


\section{Acknowledgements}

Firstly, I would like to thank my primary supervisor at Victoria University of Wellington, Nicola Nelson, who was willing to help me launch into the PhD application that brought me to New Zealand and provided advice and guidance throughout my studies. Kristine Grayson, of the University of Richmond, despite being a long-distance supervisor our Skype meetings and email exhanges were invaluable and I am thankful for her advice and meticulous feedback on numerous drafts. Brett Gartrell, of Massey University, whose knowledge, expertise and practical advice were always appreciated. I would also like to thank Nigel French, of Massey University, who welcomed me to the mEpiLab and facilitated research that would not have been possible for me otherwise. Also, Anne Midwinter, Patrick Biggs, Angie Reynolds, Zoe Grange, Lynn Rogers and everyone else at the mEpiLab who offered support, advice and assistance as I was learning the ropes.

My thanks also go to the many people at Victoria University of Wellington who have supported me, distracted me, educated me and assisted me at one point or another. Sue Keall, who always had useful advice and practical know-how. My office mates, past and present, Patty Ramirez, Anna Carter, Helen Taylor, Vaughn Stenhouse, Lindsay Anderson, Rosalynn Anderson-Lederer, Evan Brenton-Rule and Zak Murray. I would also like to thank the friends I made here and who helped make Wellington a home, in particular Brit Finucci, Annie Galland and Laina Isler, who provided endless entertainment and encouragement. Much of my fieldwork would not have been possible without the assistance of numerous volunteers and sanctuary staff who, come rain or shine, spent their days and nights on site with me. Too many of you helped over the past three years to name you all here, but thank you. Special thanks go to Emma Dent, Francesco Civilini, Te Taiawatea Moko-Mead, Emma Rowell, Colin Thompson, Noela McGregor, Sarah Alexander, Rebecca Webster, Bart Cox, John Berry, Nick Cabot and Tamsin Ward-Smith. My thanks also to the staff at ZEALANDIA, Maungatautari Ecological Island, Cape Sanctuary, Ngā Manu Nature Reserve and Ecoworks New Zealand Ltd.

Without the support of the Department of Conservation, iwi and landowners, who granted me access to the land and tuatara, none of this work would have been possible. I would like to thank Ngāti Kōata, Ngāti Mihiroa, Ngai Tāmanuhiri, the Wellington Tenths Trust, the Mana Whenua representatives for Maungatautari and Ngāti Porou. My thanks 
also go to all of the Department of Conservation staff involved in getting me to and from Whangaokeno and Stephens Island and the people who helped make my stays on these islands enjoyable ones, to Polly Hall and Hal Hovell in particular.

Funding for my PhD research was provided by several organisations and I would like to express my gratitude to the Commonwealth Scholarship Commission and Victoria University of Wellington. I also received grants from the VUW Faculty of Science, the Allan Wilson Centre, the VUW Centre for Biodiversity and Restoration Ecology, the Australasian Society for the Study of Animal Behaviour and the Society for Research on Amphibians and Reptiles in New Zealand. This research was conducted with approval from the Victoria University of Wellington Animal Ethics Committee (Permit No. 2012R33) and the New Zealand Department of Conservation (Permit No. 36857-FAU).

Final thanks are saved for my family and my partner Matt, to whom this thesis is dedicated. They supported and encouraged me in every way possible and I am unendingly grateful. I quite literally could not have done this without them. 


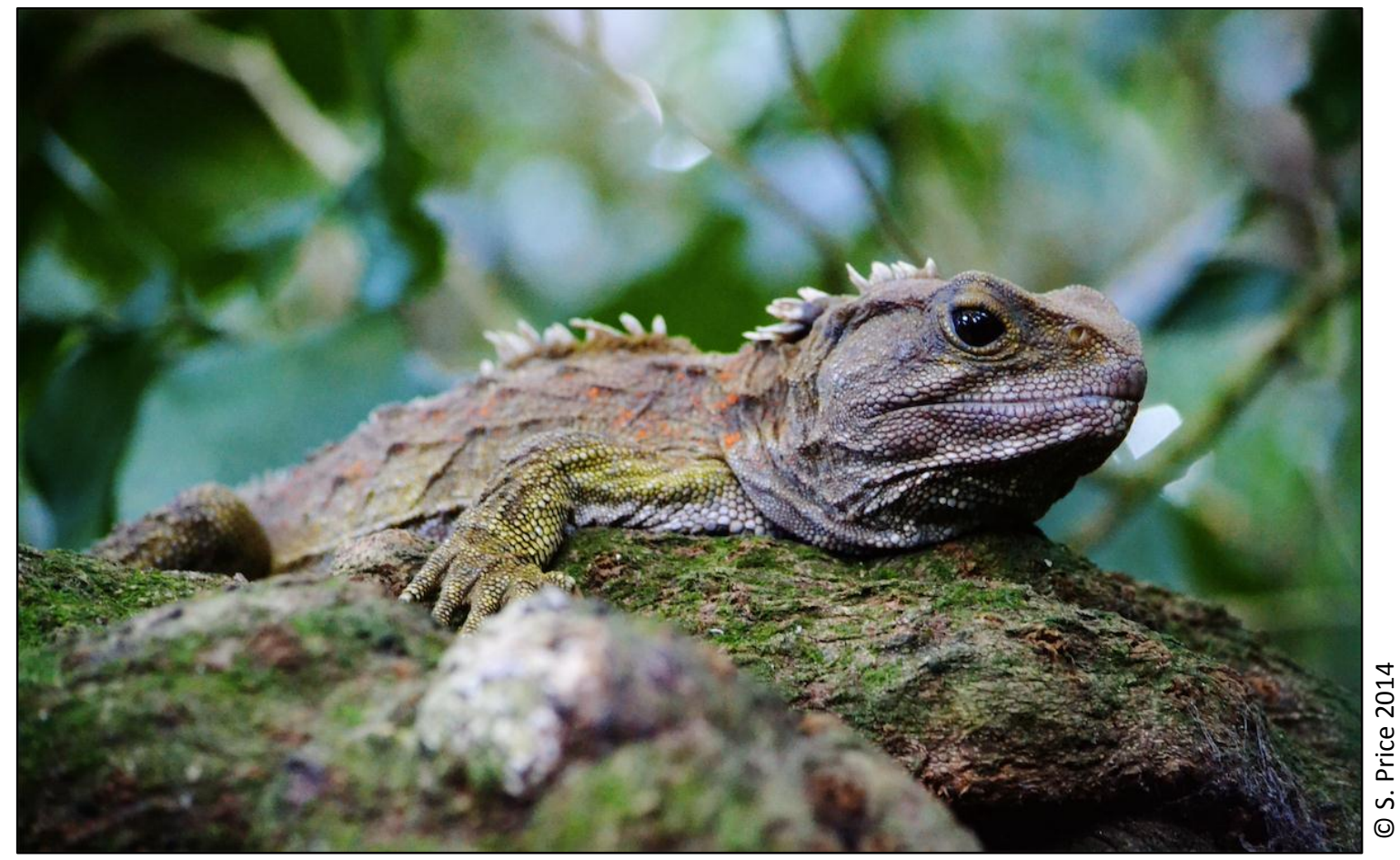

"There is a grandeur in this view of life, with its several powers, having been originally breathed into a few forms or into one; and that, whilst this planet has gone cycling on according to the fixed law of gravity, from so simple a beginning endless forms most beautiful and most wonderful have been, and are being, evolved."

- Charles Darwin, On the Origin of Species 


\section{Table of contents}

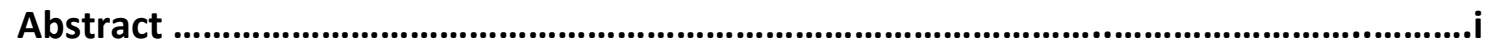

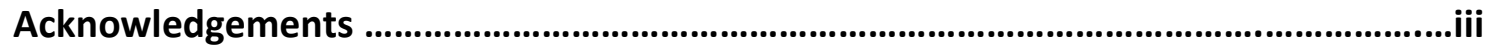

Chapter One: Using conservation translocations to assess the possible effects of anthropogenic climate change on the tuatara (Sphenodon punctatus) ..................... 1

1.1 Climate change, dispersal, adaptation and phenotypic plasticity.......................... 1

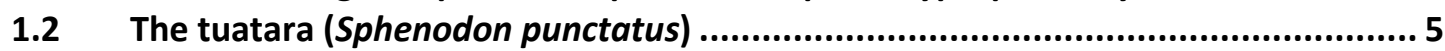

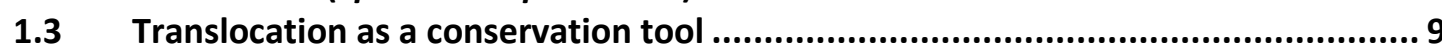

1.4 Bacteria: Commensal and potentially pathogenic microorganisms, Salmonella spp.

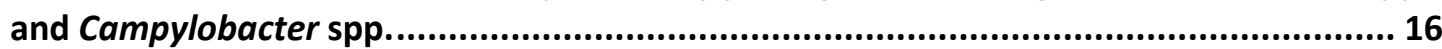

1.5 Parasites: ticks, mites, haemoparasites and intestinal parasites........................ 19

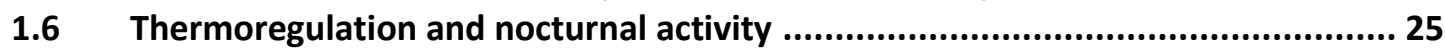

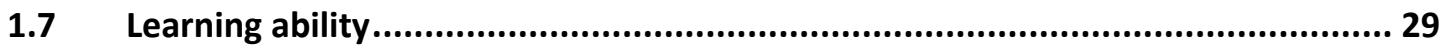

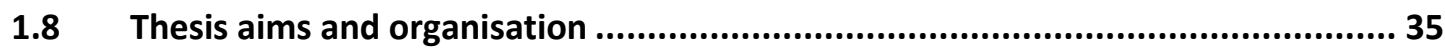

Chapter Two: Survival and growth of Cook Strait tuatara (Sphenodon punctatus) following translocation to warmer locations: implications for the temporal effects of

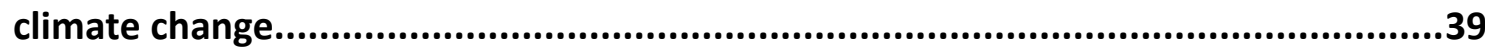

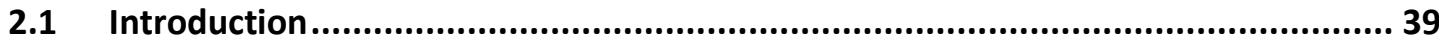

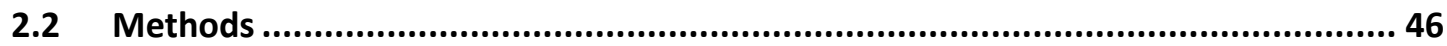

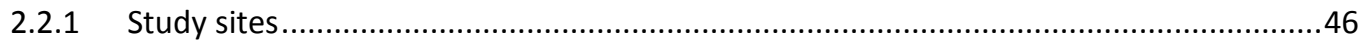

2.2.2 Founding population and translocations ............................................................. 49

2.2.3 Opportunistic monitoring ......................................................................................

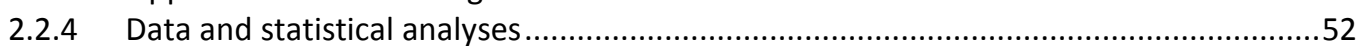

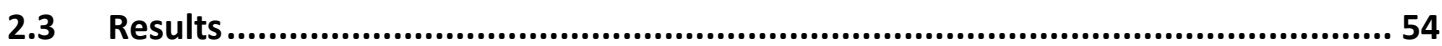

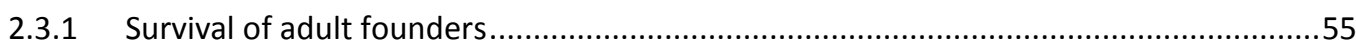

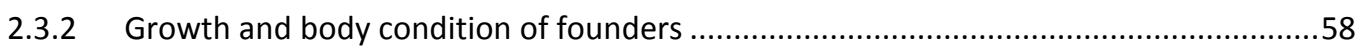

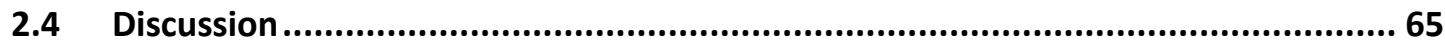

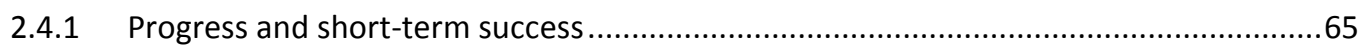

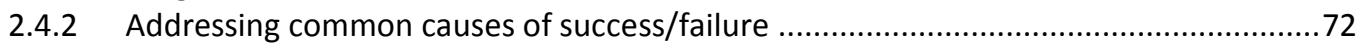

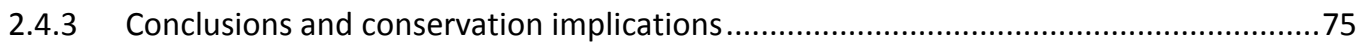

Chapter Three: The association between translocation (and associated climate shifts) and the prevalence of the gastrointestinal colonisers, Campylobacter spp. and Salmonella spp. in tuatara (Sphenodon punctatus) ................................................77

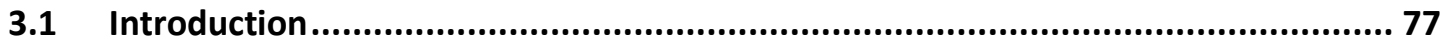

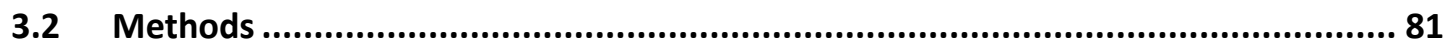

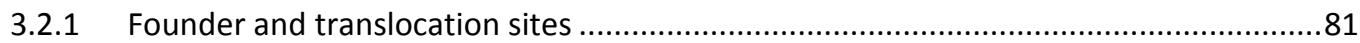

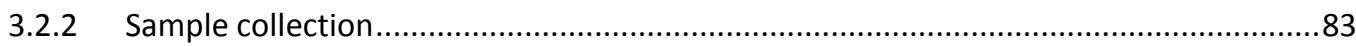

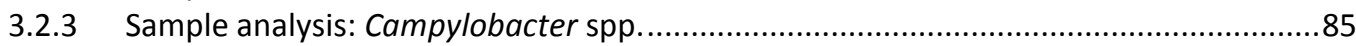

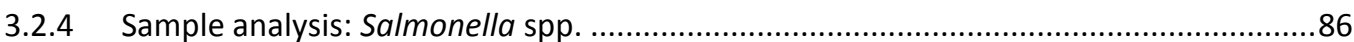

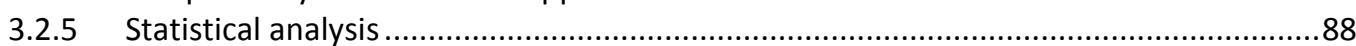

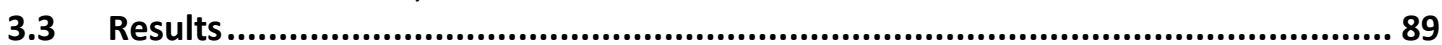

3.3.1 Campylobacter: Apparent and true prevalence ......................................................89

3.3.2 Campylobacter species confirmation and characterisation ........................................89

3.3.3 Salmonella species serovars, and apparent and true prevalence ................................90

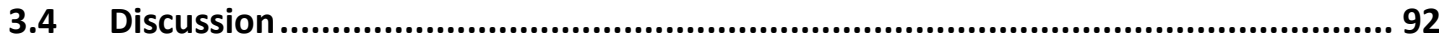


Chapter Four: Relationships between climate, population density and timing of translocation on parasites of tuatara (Sphenodon punctatus).............................. 99

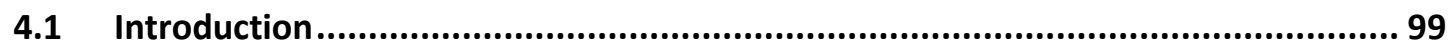

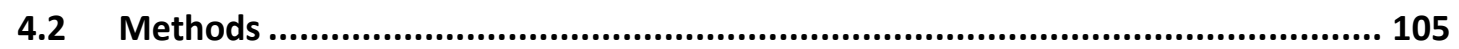

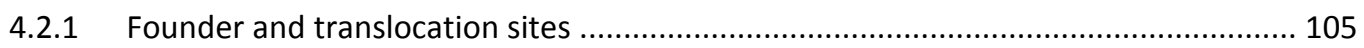

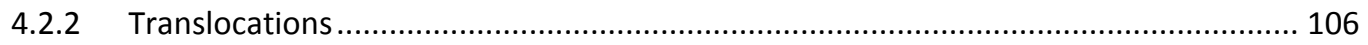

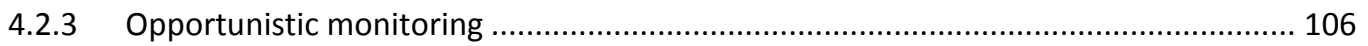

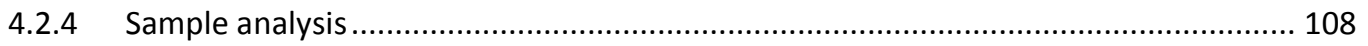

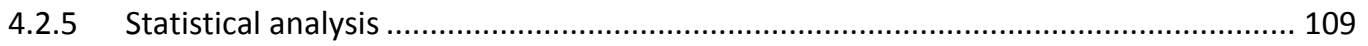

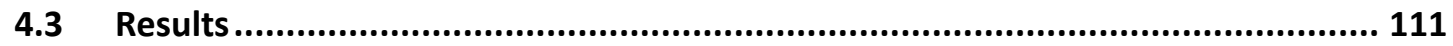

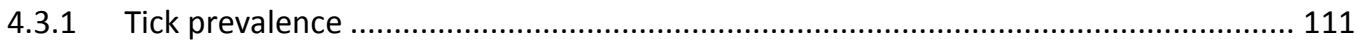

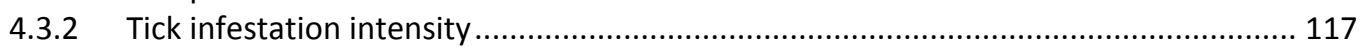

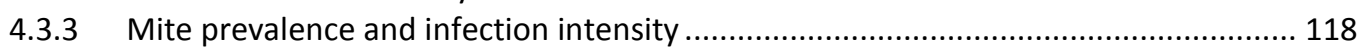

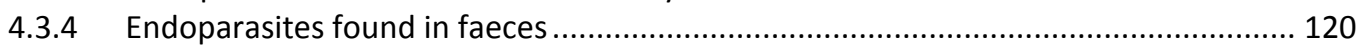

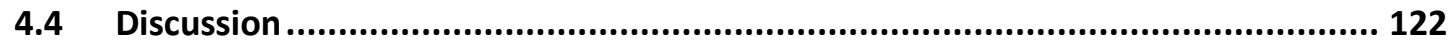

4.4.1 Tick populations following translocation ................................................................ 122

4.4.2 Mite populations following translocation ................................................................. 128

4.4.3 Occurrence of intestinal endoparasites ................................................................. 130

4.4.4 Conclusions and conservation implications ............................................................ 130

Chapter Five: Thermoregulatory behaviour and nocturnal activity in translocated populations of a cold-adapted reptile, the tuatara (Sphenodon punctatus): conservation implications with climate change ............................................... 133

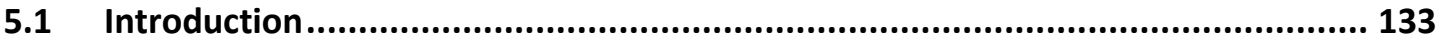

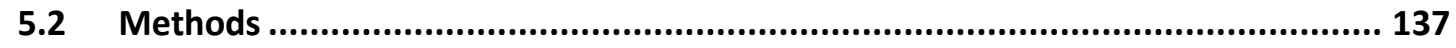

5.2.1 Founder and translocation sites ...................................................................... 137

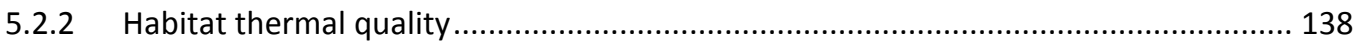

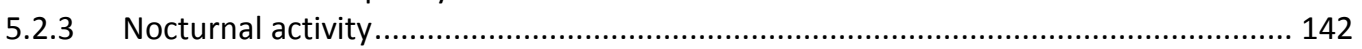

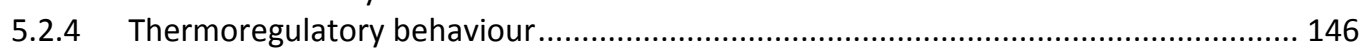

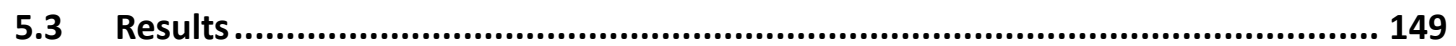

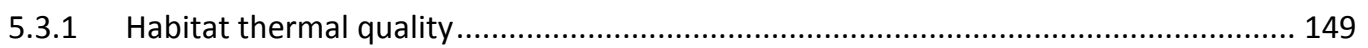

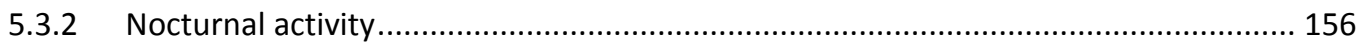

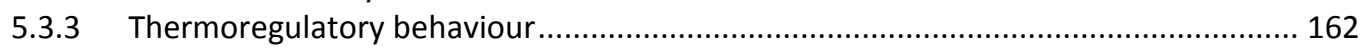

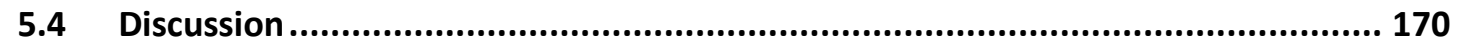

5.4.1 Environmental and skin temperatures …………................................................. 170

5.4.2 Nocturnal emergence and activity............................................................... 174

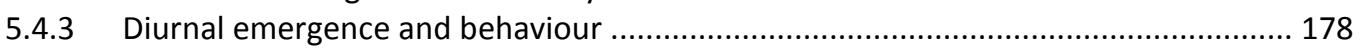

5.4.4 Conclusions and conservation implications ............................................................. 181

Chapter Six: Will a warming climate produce "smarter" reptiles? Ambient temperature and learning performance in the tuatara (Sphenodon punctatus) ........................ 185

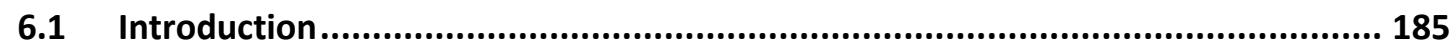

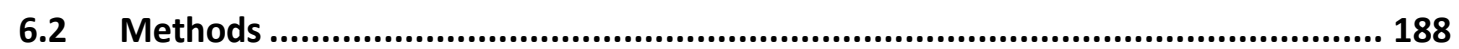

6.2.1 Study animals, husbandry and maintenance ..................................................... 188

6.2.2 Experimental setup ........................................................................................ 190

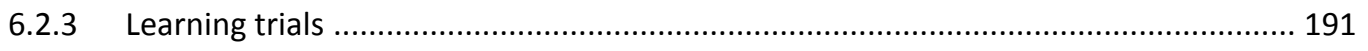

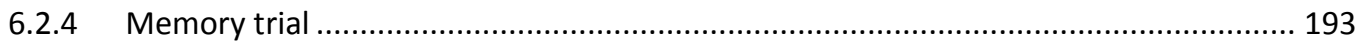

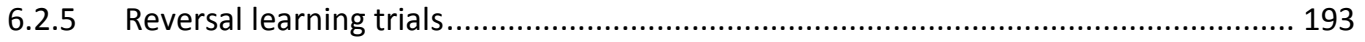

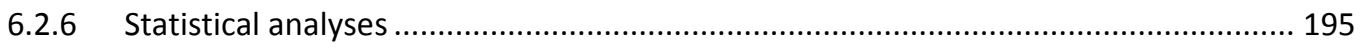

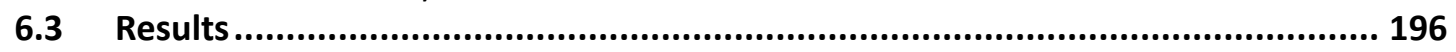

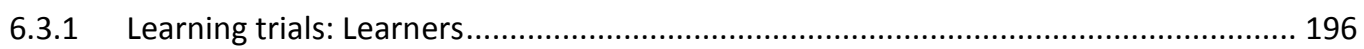

6.3.2 Learning trials: Latency and incorrect turns ......................................................... 199

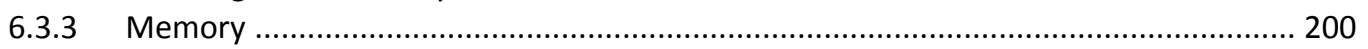

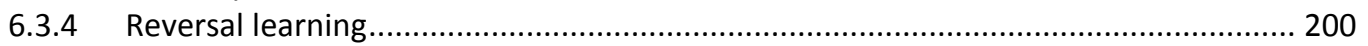




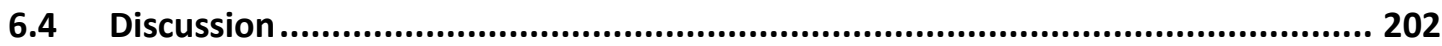

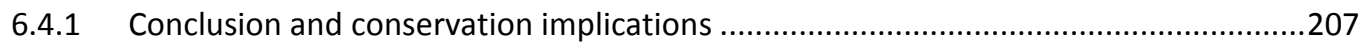

Chapter Seven: Thesis summary and discussion: Using unprecedented conservation translocations to assess impacts of anthropogenic climate change on the tuatara

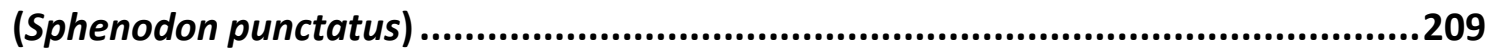

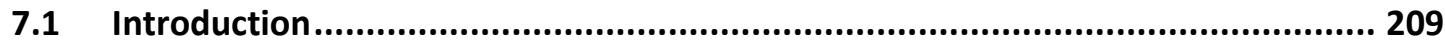

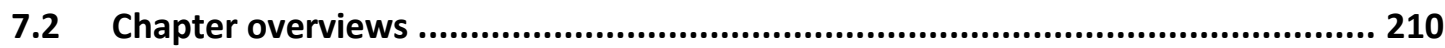

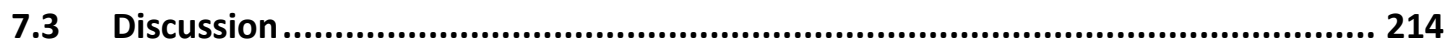

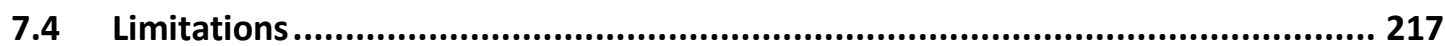

7.5 Conservation implications and management recommendations ......................... 219

7.6 Recommendations for future research......................................................... 224

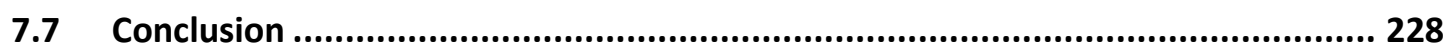

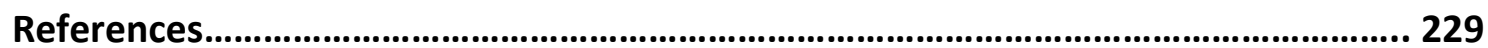

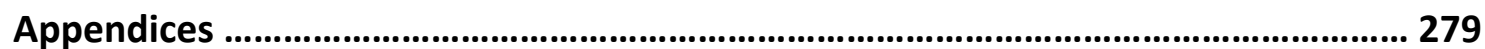




\section{Chapter One}

\section{Using conservation translocations to assess the possible effects of anthropogenic climate change on the tuatara (Sphenodon punctatus)}

\subsection{Climate change, dispersal, adaptation and phenotypic plasticity}

Environmental heterogeneity is a challenge faced by all living organisms. Environments can vary both temporally and spatially, encompassing long-term tectonic plate shifts, short-term daily and seasonal weather patterns, local variation in soil composition and global variation across a multitude of habitats and communities (Palmer \& Dixon 1990; Pigliucci 2001; Tamme et al. 2010). Sources of environmental heterogeneity can range from anthropogenic activities, like farming and development, to abiotic factors such as rainfall, cloud cover, volcanic eruptions and El Niños (Angilletta 2009). Despite the dramatic effects of major weather events, perhaps the most influential source of environmental heterogeneity is temperature.

Temperature is a ubiquitous abiotic factor that governs biological functions, cues life history events, sets range boundaries and influences disease dynamics (Ueda \& Ibuka 1995; Parmesan 2007; Angilletta 2009; Lafferty 2009; Hurlbert \& Liang 2012; ZamoraVilchis et al. 2012). The thermal sensitivities of enzymes, cell membranes and aerobic respiration results in thermal boundaries for biological reactions, beyond which an organism may not die, but will cease to function normally (Stein et al. 1982; Somero 1995; Somero et al. 1996; DeCoursey \& Cherny 1998; Pörtner 2002; Angilletta 2009). Consequently, functions like growth, feeding, reproduction and locomotion in animal taxa are reliant on suitable body temperatures (Bennett 1978; Johnston \& Bennett 1996; Angilletta 2009). If body temperatures exceed an animal's thermal maximum, protein stability can be compromised, the conformation of enzymes and membranes can be disrupted or destroyed, and oxygen-limitation can see mitochondria fail to produce sufficient ATP (Frederich \& Pörtner 2000; Angilletta 2009). Accordingly, at critically high temperatures biochemical reactions will slow, energetic costs will not be met, physiological function will be impaired and, ultimately, biological function may cease completely (Somero 1995; Angilletta 2009). As such, the biochemical constraints, 
preferred temperatures, and acclimatory or adaptive capacity of organisms to local climates has led to the establishment of populations in thermal niches that support biological functioning (Johnston \& Bennett 1996). An example of this lies in Antarctic icefish (Notothenioidei), which are extreme stenotherms surviving within a very limited, cold tolerant temperature range of $6^{\circ} \mathrm{C}\left(-1.9-4^{\circ} \mathrm{C}\right.$; Somero \& DeVries 1967; Somero et al. 1996). This high degree of specialisation can be energetically beneficial, however such a narrow thermal tolerance range and consequently limited dispersal ability means that stochastic events and rising water temperatures pose a substantial threat to the icefish (Somero et al. 1996; Logan \& Buckley 2015). In contrast, the eurythermic Crusian carp (Carassius carassius) can tolerate a broad temperature range of $0-36^{\circ} \mathrm{C}$, allowing it to access a wider range of habitats and conferring greater tolerance of unpredictable or changing thermal environments (Kakela et al. 2008; Logan \& Buckley 2015). Such adaptive differences contribute to the geographical distribution of species observed worldwide (Somero et al. 1996).

The rate at which anthropogenic climate change is proceeding is a significant conservation concern (IPCC 2014). It is predicted that global atmospheric temperatures could increase by $0.3-0.7^{\circ} \mathrm{C}$ by 2035 and up to $4.8^{\circ} \mathrm{C}$ by 2100 (based on highest and lowest estimates from the CMIP5 model simulations RCP2.6 and RCP8.5), with more rapid increases in surface temperature expected at the poles (IPCC 2014). The diurnal temperature range is also narrowing due to asymmetric changes in minimum and maximum daily temperatures, with minimum temperatures rising at faster rates or decreasing at slower rates than daily maximum temperatures (Karl et al. 1991, 1993; Easterling et al. 1997). The global environmental impacts of climate change thus far have been widespread and include increased drought, flooding, sea level rise, habitat loss/fragmentation, sea ice reduction, glacial retreat, and cyclone intensification (Pounds et al. 1999; Hughes et al. 2003; Mawdsley et al. 2009; IPCC 2014; Reisinger et al. 2014). Within New Zealand it is predicted that air temperatures will increase by $0.3-1.4^{\circ} \mathrm{C}$ by the 2040s and $0.7-5.1^{\circ} \mathrm{C}$ by the $2080 \mathrm{~s}$, with associated regional changes in the frequency of heavy rainfall, reductions in soil moisture, and increases in the incidence of severe drought and fire danger (Hennessy et al. 2007; Reisinger et al. 2014). Such stressors, in conjunction with rapid temperature increases, are expected to have significant impacts on biodiversity, with population declines, extirpations, extinctions, range 
contractions/expansions/shifts, competitive invasions, increasingly asynchronous interactions, disruptions in the timing of life-history events and the spread of disease already being documented (Pounds et al. 1999; Root et al. 2003; Parmesan 2006; Visser 2008; Mawdsley et al. 2009; Hoffman \& Sgro 2011; Hoffman et al. 2013; Quintero \& Wiens 2013). Current predictions anticipate such effects escalating as climate change progresses (Angilletta 2009; IPCC 2014; Reisinger et al. 2014).

Endothermic or "warm-blooded" species are able to maintain relatively constant body temperatures due to high metabolic rates and behavioural processes, which buffer them to some degree against suboptimal environmental temperatures (Clarke \& Pörtner 2010). In contrast, while many ectotherms (e.g. reptiles, invertebrates, fish, amphibians) can behaviourally thermoregulate to maintain body temperatures within their activity temperature range, they are ultimately dependent on solar radiation and heat absorption from their surroundings and are thus more vulnerable to heterogeneity in the thermal environment and rising temperatures predicted under climate change (Cowles \& Bogert 1944; Hochachka \& Somero 2002).

Biological fitness is broadly defined as the ability of an organism to survive to reproductive age, reproduce, and contribute to the next generation (Orr 2009). Deutsch et al. (2008) proposed that ectothermic animals at mid to high latitudes might experience initial fitness benefits from temperature increases due to their broader thermal tolerances and the occupation of climates generally below their thermal optima, whereas those already living at temperatures close to their upper thermal limits (e.g. tropical species), are likely to suffer with even small increases in temperature. Conversely, other authors have suggested that mid to high latitude $\left(20-40^{\circ}\right)$ ectotherms will be more likely to experience thermal stress due to the higher temperature increases predicted at these locations, lower tolerances to high temperatures, and a limited potential for acclimation of their critical thermal limits (Calosi et al. 2008; Hoffman \& Sgro 2011; Hoffman et al. 2013). Nonetheless, as climates continue to warm environmental temperatures will likely approach the thermal tolerance limits of even the more robust species and these too will suffer (Deutsch et al. 2008).

Three mechanisms by which species might circumvent the risk of extinction have been postulated. Organisms could 1) phenotypically adjust to the changing climate, 2) genetically adapt through microevolution, or 3) physically disperse to thermally suitable 
habitats (Hoffman \& Sgro 2011; Quintero \& Wiens 2013). However, some species are more at risk than others and the options for mitigating threats associated with anthropogenic climate change may not be achievable for many species and populations. Throughout evolutionary history organisms have had to adapt to environmental change to survive and it is expected that future changes will again drive evolutionary adaptive responses (Mawdsley et al. 2009). However, successful adaptation to the unprecedented rates of current climate change will depend on the ability of organisms to change quickly enough that a maladaptive lag between environmental conditions and phenotype does not occur and it is expected that populations with high genetic diversity, short life spans, and high reproductive rates will have a greater capacity for rapid adaptation (Angilletta 2009; Hoffman \& Sgro 2011; Refsnider \& Janzen 2012; IUCN/SSC 2013). Conversely, the rate of climate change is likely to exceed the rate at which long-lived species with long generation times are able to adapt through genetic change (Nelson et al. 2004b; Refsnider \& Janzen 2012). Hoffman and Sgrò (2011) postulate that lags between environmental conditions and optimal phenotypes could theoretically lead to faster evolutionary responses due to intense selection on maladapted traits. However, rapid adaptation under high selection pressure can reduce genetic variation, which can prevent adaptation to future environmental variation and increase extinction risk (Hoffman \& Sgro 2011; Refsnider \& Janzen 2012).

The ability to disperse to climatically suitable habitats is critical for species that are unlikely to adapt to or physiologically compensate for rising air temperatures. Range shifts have been documented in recent years as populations track suitable climates and shift their distributions to higher latitudes and altitudes (Parmesan 2006; Calosi et al. 2008; Mawdsley et al. 2009; Chen et al. 2011; Walther et al. 2012). However, dispersal is not always a viable option and specialist, range-restricted (e.g. mountaintop species), isolated, immobile or poorly dispersing species (e.g. coral symbionts) may be unable to relocate to suitable habitats and there are potential fitness consequences associated with shifts in distribution, including exposure to disease, competitive exclusion, and conflict (Kutz et al. 2005; Parmesan 2006; Atkins \& Travis 2010; Jankowski et al. 2010; Refsnider 2013).

Phenotypic plasticity is the ability of a genotype to generate a variety of phenotypes in response to environmental variation and is considered one of the main 
ways in which organisms can react to climate change (Chown et al. 2007; Nussey et al. 2007; Whitman \& Agrawal 2009; Richter et al. 2012). A change in the phenotype can entail a change in phenology, morphology, behaviour, or physiology, which can subsequently affect colouration, locomotor function, thermoregulation, growth, reproduction, etc, and can improve fitness in variable environments (Whitman \& Agrawal 2009). Numerous species, from ectotherms to endotherms, arthropods to mammals, across marine and terrestrial habitats have been found to exhibit such plasticity (Ford \& Seigel 1989; Johnston 1993; Nager \& van Noordwijk 1995; Réale et al. 2003; Chown et al. 2007; Nussey et al. 2007; Charmantier et al. 2008; Menegaz et al. 2009; Aubret \& Shine 2009; Clusella Trullas et al. 2010; Sun \& Niu 2012). A plastic response differs from genetic adaptation (a change in the genotype) because it involves the environmentally sensitive production of different phenotypes from the same genotype and can occur both within and between generations (DeWitt \& Scheiner 2004; Whitman \& Agrawal 2009). Phenotypic plasticity does not facilitate an immediate response and there is still potential for maladaptive lags to occur; however, if rapid enough this ability can facilitate short-term change and allow an organism to keep pace with environmental variation, facilitating improved performance and potentially enhancing fitness (DeWitt \& Scheiner 2004; Nussey et al. 2007; Angilletta 2009). As such, phenotypic plasticity is a key mechanism by which species that are slow to reproduce or unable to disperse may adjust to climate change (Refsnider \& Janzen 2012; Richter et al. 2012; Urban et al. 2014).

\subsection{The tuatara (Sphenodon punctatus)}

The tuatara is a long-lived ( 100 years), nocturnal, cold-adapted, New Zealand endemic reptile and the sole extant representative of the Order Rhynchocephalia, which arose around 250 million years ago in the late Triassic (Saint Girons 1980; Barwick 1982; Dawbin 1982; Cree \& Butler 1993; Evans et al. 2001). Tuatara were “on board" New Zealand when it separated from the supercontinent Gondwana 80 million years ago and thus survived the upheaval of land masses, volcanic events and periods of past climate change like the "Oligocene drowning" (Gibbs 2006; Jones et al. 2009; Miller et al. 2012). While their ancestors were able to survive through past climatic change there is a lack of information 
on how physiologically similar extant populations are to their extirpated ancestors so we cannot assume that tuatara have retained attributes that previously enabled their ancestors to persist (Miller et al. 2012). As such, the impact that contemporary climate change might have on extant tuatara populations is largely unknown and assessments of multiple aspects of tuatara life history (e.g. physiology, disease ecology, parasitology, reproduction, behaviour, cognition) following exposure to warmer climates will be vital to predicting if and how this species might persist.

Originally thought to be a member of the Agamidae and named "Hatteria punctata" in 1842, the British Museum's curator of reptiles, Albert Günther, reassigned the tuatara to the Order Rhynchocephalia, a sister taxon to the Squamata (lizards, snakes and amphisbaenids), in 1867, making the tuatara the only extant non-squamate Lepidosaur (Gray 1842; Günther 1867). While tuatara may superficially resemble lizards they possess several distinct anatomical characteristics that set them apart, including uncinate processes on the ribs, gastralia (abdominal ribs), the lack of a male copulatory organ and unique shearing teeth (Günther 1867; Robb 1977). Due to its long evolutionary history and retention of some ancestral features (e.g. the parietal foramen, an opening in the skull above the parietal eye or "third" eye) the tuatara has often been referred to as a "living fossil," though they are well adapted to the New Zealand environment and many of the tuatara's features have been found to be derived, rather than primitive (Daugherty \& Cree 1990; Jones et al. 2009; Cree 2014).

Holocene subfossil records indicate that tuatara were once widespread throughout New Zealand (Figure 1.1), but with the arrival of Polynesian and European settlers (around AD 1250-1300 and AD 1769 respectively) came human-mediated habitat change, hunting and introduced mammalian predators (e.g. rats, cats, stoats; Cree \& Butler 1993; Worthy \& Holdaway 2002; Wood 2009; Cree 2014). Prior to human arrival the predators of adult tuatara would have been entirely avian (e.g. laughing owls (Sceloglaux albifacies), adzebills (Aptornis spp.)) and the evolution of strategies to avoid predation by primarily visual hunters (e.g. camouflage, immobility) would have left tuatara vulnerable to scent-guided mammalian predators (Worthy \& Holdaway 2002; Cree 2014). Tuatara were consequently extirpated from the mainland and are now restricted to approximately 31-35 offshore islands around New Zealand, the majority of which are pest-free and serve as refuges to protect tuatara against further decline (Gaze 
2001; MacAvoy et al. 2007; Towns et al. 2007; Miller et al. 2012; Cree 2014). Despite such a dramatic impact on tuatara populations, the Australasian Reptile \& Amphibian Specialist Group contribution to the IUCN Red List assessed tuatara to be a species of low risk/least concern, likely due to the presence of several large populations (e.g. the $\sim 30,000$ to 50,000 individuals on Stephens Island), and this classification has remained unchanged since 1996 (Newman 1982; Gans 1983; ARASG 1996). One the other hand, the New Zealand Department of Conservation Threat Classification System considers the tuatara to be conservation dependent, range restricted and relict, having undergone a population decline in the past 1000 years and currently occupying less than $10 \%$ of their former range (Miller et al. 2012; Cree 2014; Hitchmough et al. 2016).

Tuatara populations are particularly at risk from the rising temperatures associated with anthropogenic climate change. Tuatara are considered to be a coldadapted species, with a preferred body temperature range of $19.5-23.1^{\circ} \mathrm{C}$ (Besson \& Cree 2010, 2011; Corkery et al. 2014a), an active body temperature range of $5-30^{\circ} \mathrm{C}$ (Barwick 1982; Walls 1983; Thompson \& Daugherty 1998), and a possible upper body temperature tolerance threshold of $28-30^{\circ} \mathrm{C}$ (Barwick 1982; Heatwole 1982). However, a body temperature of $34.5^{\circ} \mathrm{C}$ has been recorded in the wild (Corkery 2012), and captive tuatara have been observed to suffer heat stress and death at air temperatures above $25^{\circ} \mathrm{C}$ (Blanchard 2002), so there is variation around the upper threshold, which is likely dependent on the amount of time spent at these temperatures. Likewise, relative humidity and wind have been observed to influence the emergence and activity of tuatara (Walls 1983; Corkery et al. 2014a). The maintenance of such low body temperatures would be deadly to many other reptile species and attainment of the warmer body temperatures associated with many other species would be highly detrimental to tuatara, indicating that tuatara are well adapted to the temperate New Zealand climate (Brattstrom 1965). Tuatara also have a unique form of temperature-dependent sex determination (TSD), a process by which temperature during embryonic development determines the sex of the organism (Cree et al. 1995; Nelson et al. 2004a). The rare form of TSD (type 1b) found in tuatara means that $100 \%$ female offspring are produced in cooler nests $\left(\leq 21.2^{\circ} \mathrm{C}\right)$ and $100 \%$ males are produced in warmer nests $\left(>22.2^{\circ} \mathrm{C}\right.$; Nelson et al. 2004a; Mitchell et al. 2006). As the transitional range between all-male and all-female clutches is only $1^{\circ} \mathrm{C}$ and males are produced at higher temperatures, this mode of TSD and 
narrow transitional range may put populations at risk of significant male sex ratio bias as the climate warms and could ultimately lead to population extinctions (Nelson et al. 2004b; Miller et al. 2012).

The long-term goal of the New Zealand Department of Conservation's Tuatara Recovery Group was to maintain the genetic diversity of tuatara by reinstating wild populations throughout their historical range and restoring existing tuatara populations to their pre-human states (Gaze 2001). As many populations survive on isolated offshore islands from which dispersal to the mainland is not feasible, and as the initial cause of decline (i.e. introduced mammals) has not been eliminated from New Zealand, humanmediated translocations to predator-free locations have been essential for tuatara conservation (Cree \& Butler 1993). The spread of introduced mammals and consequent decline of isolated island-dwelling tuatara populations led Daugherty et al. (1992) to argue that legal protection of tuatara alone would not be sufficient to see the species persist and that active conservation was required (Crook 1973; Towns et al. 2001). The first contemporary conservation translocation of tuatara took place in 1995 when adult and juvenile animals were moved from North Brother Island to Titi Island within New Zealand's Cook Strait (Nelson 1998). Since then, further translocations have been made to several islands within the tuatara's historical range from which predatory mammals have been eradicated (Towns et al. 2001; Nelson et al. 2002b; Gartrell et al. 2006). Because tuatara are long-lived and slow to reproduce even early translocations have not yet reached the stage at which population viability, and ultimately translocation success, can be ascertained. While recent reports on short-term success are not available for all tuatara translocations, in several cases post-release monitoring has shown populations to be progressing favourably. Multiple monitoring trips in the 9-11 years following the translocations of tuatara from North Brother Island to Titi Island and Matiu/Somes Island recaptured over half of the founding populations, found that all had gained weight postrelease, and discovered evidence of reproduction on both islands (Nelson 1998; Nelson et al. 2002b; Miller et al. 2010). Similarly, a translocation of wild-caught adults from Moutoki to Moutohora/Whale Island in 1996 and a translocation of wild caught adults and wild caught/head-started juveniles to Wakatere-papanui in 2003/2004 were found to be progressing well, with recaptured animals showing increases in size and evidence of 
reproduction found in the Moutohora population (Owen 1998; MacBrayne 2002; Gaze \& Cash 2008; Nelson et al. 2008).

Tuatara could theoretically adapt to a warming climate by shifting their thermal sensitivity boundaries, adjusting their mode of TSD or by dispersing to cooler climates (Huey \& Janzen 2008; Besson \& Cree 2011). However, their slow sexual maturation and long reproductive cycles suggest that any genetic adaptation would likely occur at too slow a rate to improve survival (Nelson et al. 2004b; Refsnider \& Janzen 2012). Nor is dispersal a likely option due to the restriction of tuatara to offshore islands (Nelson et al. 2002b). This makes understanding the responses to warmer climates vital for direct conservation management efforts and to assure the future of the species. Translocation is an essential tool for tuatara conservation and recent translocations to mainland sites serve to restore tuatara to locations within their former range and offer the opportunity to study potential responses to climate change, as well as aid conservation targets and public awareness goals (Gaze 2001; Nelson et al. 2002b; Miller et al. 2012).

\subsection{Translocation as a conservation tool}

As defined by the International Union for Conservation of Nature (IUCN), a translocation is the "human mediated movement of living organisms from one area, with release in another" (IUCN/SSC 2013). Translocations of animals and plants, both intentional and accidental, have transpired since the Pleistocene when humans first began colonising new lands (Seddon et al. 2012). With direct impacts of anthropogenic climate change observed from the local population to global biodiversity level and additional threats imposed by other human activities (e.g. habitat loss/fragmentation, hunting), vulnerable species are at growing risk of local and global extinction (Pounds et al. 1999, 2006; Cullen et al. 2000; Root et al. 2003; Travis 2003; Parmesan 2006; Walther et al. 2012). As such, translocation can be a valuable conservation tool that can remove animals from unsuitable habitats, extend a species' range or supplement/restore small or extirpated populations, ultimately reducing the likelihood of species extinction (Griffith et al. 1989; Dodd \& Seigel 1991; Parmesan 2006; Germano \& Bishop 2008; Mawdsley et al. 2009; Thomas 2011; Miller et al. 2012; IUCN/SSC 2013). 
A translocation is considered successful when it results in a self-sustaining wild population and successes have been documented for a wide variety of taxa, including birds, mammals, reptiles, invertebrates and plants, demonstrating that this conservation strategy can prove highly beneficial (Burke 1992; Sanz 1998; Spalton et al. 1999; Maschinski \& Duquesnel 2007; US Fish And Wildlife Service 2008; Thomas et al. 2009; Soorae 2010, 2011, 2013; Seddon et al. 2012). However, translocations have historically focussed on birds and mammals, with herpetofauna accounting for a much smaller proportion of published translocation research (Fischer \& Lindenmayer 2000; Seddon et al. 2005). Herpetofauna were for a while considered unsuitable for translocation after a review by Dodd and Seigel (1991) found that only $19 \%$ of reviewed translocations could be deemed successful, the majority of which were crocodilian species. This low success rate was attributed to a lack of long-term monitoring ( $58 \%$ of translocations could not be classified due to inadequate data), insufficient understanding of the original cause of species decline, and a lack of attention to biological constraints and population genetics (Dodd \& Seigel 1991). The importance of addressing the cause of species decline and need for long-term monitoring was further highlighted in a recent review of lizard translocations in New Zealand (Romijn and Hartley 2016). The review found that mitigation-driven translocations often released animals into areas where mammalian predators were still present and that long-term monitoring was lacking in both conservation and mitigation-driven translocations (Romijn and Hartley 2016). Other studies have also highlighted disease, stress, and reduced reproductive ability as contributors to translocation failure (Seigel \& Dodd 2002; Mackintosh \& Briskie 2005; Teixeira et al. 2007; Pinter-Wollman et al. 2009).

A review by Germano and Bishop (2008) found that global success rates for herpetofauna translocations have more than doubled to $41 \%$, with the most common reasons for failure now being homing or migratory behaviour and release into inadequate habitat. Another review also found that $60 \%$ of reptile translocation case studies had been categorised as "successful" or "highly successful" (Ewen et al. 2014).

While there has evidently been an improvement, it is still apparent that around half of herpetofauna translocations are either inadequately monitored or fail outright to establish viable populations (Fischer \& Lindenmayer 2000; Germano \& Bishop 2008; Miller et al. 2014). Following on from this, recent work on the criteria used to determine 
translocation outcomes found that reported successes in New Zealand were grossly overestimated when using a traditional definition (recruitment to the adult population observed over long-term monitoring) versus standardised success rates (involving four outcome stages that could be applied over different time scales in accordance with the species life history and time since release; Miller et al. 2014). The disparity in success rates according to the different definitions (traditional: 41.7\%; standardised: $8.1 \%$ ) highlights a need for comprehensive standardised assessment criteria that account for species longevity and encourages the reporting of inconclusive outcomes, especially where populations of long-lived or slowly reproducing species have been monitored for a short time (Miller et al. 2014). For example, a translocation of gopher tortoises (Gopherus polyphemus) in the 1980 s defined translocation success as the animals' use of a preprepared burrow at the release site, which is far removed from the post-release monitoring now recommended (Lohoefener \& Lohmeier 1986; Fischer \& Lindenmayer 2000; Germano \& Bishop 2008). Similarly, the failure of a 1978 translocation of giant land tortoises (Geochelone gigantea) in the Seychelles archipelago illustrates the limited scope of short-term data. Though the translocation was initially considered successful, over a decade later it was declared a failure after researchers observed a continual population decline (Stoddart et al. 1982; Samour et al. 1987; Hambler 1994; Fischer \& Lindenmayer 2000).

As long lifespans, slow sexual maturation and long reproductive cycles are characteristic of many herpetofauna species it has been advised that translocated populations be monitored for at least the time required for an individual to reach sexual maturity before population viability can be accurately evaluated (Germano \& Bishop 2008). It is also critical that potential complications (e.g. predators, disease, dispersal) are detected early therefore regular monitoring is necessary. The establishment of well defined success criteria, careful planning, long-term monitoring and the publication of failures/negative results are key to accurate, useful post-translocation reporting. (Dodd \& Seigel 1991; Fischer \& Lindenmayer 2000; Towns et al. 2001; Germano \& Bishop 2008; McCoy et al. 2014; Miller et al. 2014). Four stages have been established to assess translocation success, the first two of which can be used as indicators of short-term translocation progress. These are: 1) the survival and growth of founders, 2) evidence of reproduction, 3) population growth through recruitment of locally born offspring, and 4) 
a self-sustaining population (IUCN/SSC 1998; Nelson et al. 2002b; Germano \& Bishop 2008; Seddon et al. 2012; Miller et al. 2014).

To maximise the chance of translocation success it is vital that the initial cause of species decline is addressed (Dodd \& Seigel 1991). Regarding translocations of reptiles within New Zealand this often involves the eradication of invasive mammals from the release site, as well as the use of pest-proof fences and/or rigorous trapping regimes to keep mammal populations at non-existent or manageable levels. It is also advised that a sufficient number of individuals (to avoid negative Allee effects) must be released into high-quality habitat, preferably within the species historical range and at a site lacking potential competitors (Griffith et al. 1989; Dodd \& Seigel 1991; Wolf et al. 1996; Fischer \& Lindenmayer 2000; Germano \& Bishop 2008). The source of the founding population can likewise influence a translocations outcome and there are risks associated with local adaptation, age, and genetic variation (Dodd \& Seigel 1991; Miller et al. 2012). Disease screening is also essential to minimise the potential for translocated animals to introduce pathogens into naïve local populations (Leighton 2002; Gartrell et al. 2006, 2007; Germano \& Bishop 2008; Kock et al. 2010). Wild caught individuals constitute the majority of reptile translocations, which has been associated with an increased chance of translocation success (Griffith et al. 1989; Fischer \& Lindenmayer 2000). However, a review by Germano and Bishop (2008) was unable to confirm this association due to uncertain outcomes associated with translocations of captive animals at the time of the review, though they found that the source of translocated amphibians had no effect on translocation success.

An alternative to the capture of exclusively wild individuals is the use of headstarted animals, which are captive-bred or wild-caught and raised in captivity until release (Alberts 2007). Headstarting aims to improve the survival of species with high juvenile mortality by boosting the growth rate and body size of juveniles during their most vulnerable life stage, thereby improving the likelihood that individuals survive to sexual maturity (Haskell et al. 1996; Alberts 2007). Although there are disadvantages to using captive-bred individuals (e.g. the potential loss of natural behaviours), many reptile species are considered ideal owing to cost-effective production, high fecundity, a lack of parental care, and general retention of innate behavioural and physiological traits (Nelson et al. 2002b; Germano \& Bishop 2008). In the case of herpetofauna, larger individuals tend 
to have higher survivorship post-release and juvenile tuatara survival has shown to be enhanced by headstarting (Gruber 2007). The increased hatching success of artificially incubated vs. wild incubated eggs also means that artificial incubation can reduce pressure on the source population as a greater number of individuals can be obtained for translocation with minimal removal of eggs (Thompson 1990; Cree et al. 1995; Thompson et al. 1996; Nelson et al. 2004b). Translocating populations that are composed of both head-started juveniles and adult animals can be advantageous due to a reduced vulnerability to predation and unfavourable environmental conditions, which can reduce mortality in founding populations (Haskell et al. 1996; Alberts 2007; Germano \& Bishop 2008).

"Humane considerations" for the welfare and perpetuation of a species are often the driving factors behind translocations, and the majority of reptile translocations (74\%) are done in the interest of species conservation (Germano \& Bishop 2008). Such translocations are strictly governed by conservation agencies and should adhere to IUCN guidelines regarding design, management, feasibility, and threat analysis and reporting. It is perhaps for this reason that conservation-motivated translocations are the most successful and over 400 conservation translocations have been conducted in New Zealand alone (Armstrong \& McLean 1995; Germano \& Bishop 2008). In contrast, mitigationdriven translocations, which aim to reduce human activity-associated wildlife mortality (e.g. move animals away from development sites), are often well-funded but poorly prepared and executed and, consequently, the most likely to fail (Dodd \& Seigel 1991; Germano \& Bishop 2008; Germano et al. 2015). In cases like these the welfare of the translocated animals is unlikely to be a priority, a species' ecological and behavioural requirements may be disregarded, release sites may not be carefully selected, commensal species may be overlooked, translocations may be poorly orchestrated/undocumented and post-release monitoring, when performed, is often insufficient or may neglect to report failures for fear of negative publicity (Dodd \& Seigel 1991; Teixeira et al. 2007; Germano et al. 2015). Nevertheless, translocations in general can provide research opportunities, benefit vulnerable species, stimulate positive publicity, raise public awareness of conservation issues and influence conservation attitudes, which may consequently generate funding to support further conservation work (Dodd \& Seigel 1991; Germano \& Bishop 2008; Seddon et al. 2012). 
In October 2012 several conservation translocations moved 176 adult tuatara from Takapourewa/Stephens Island in the Cook Strait to four sites around New Zealand's North Island, all of which lie within the species' historical range: Maungatautari Ecological Reserve, Whangaokeno/East Island, Cape Sanctuary and Young Nicks Head Sanctuary (Figure 1.1; Worthy \& Holdaway 2002; Wood 2009). Another translocation, conducted at the same time, also moved adult and juvenile tuatara from Takapourewa/Stephens Island to Orokonui Ecosanctuary in New Zealand's South Island. This population is being

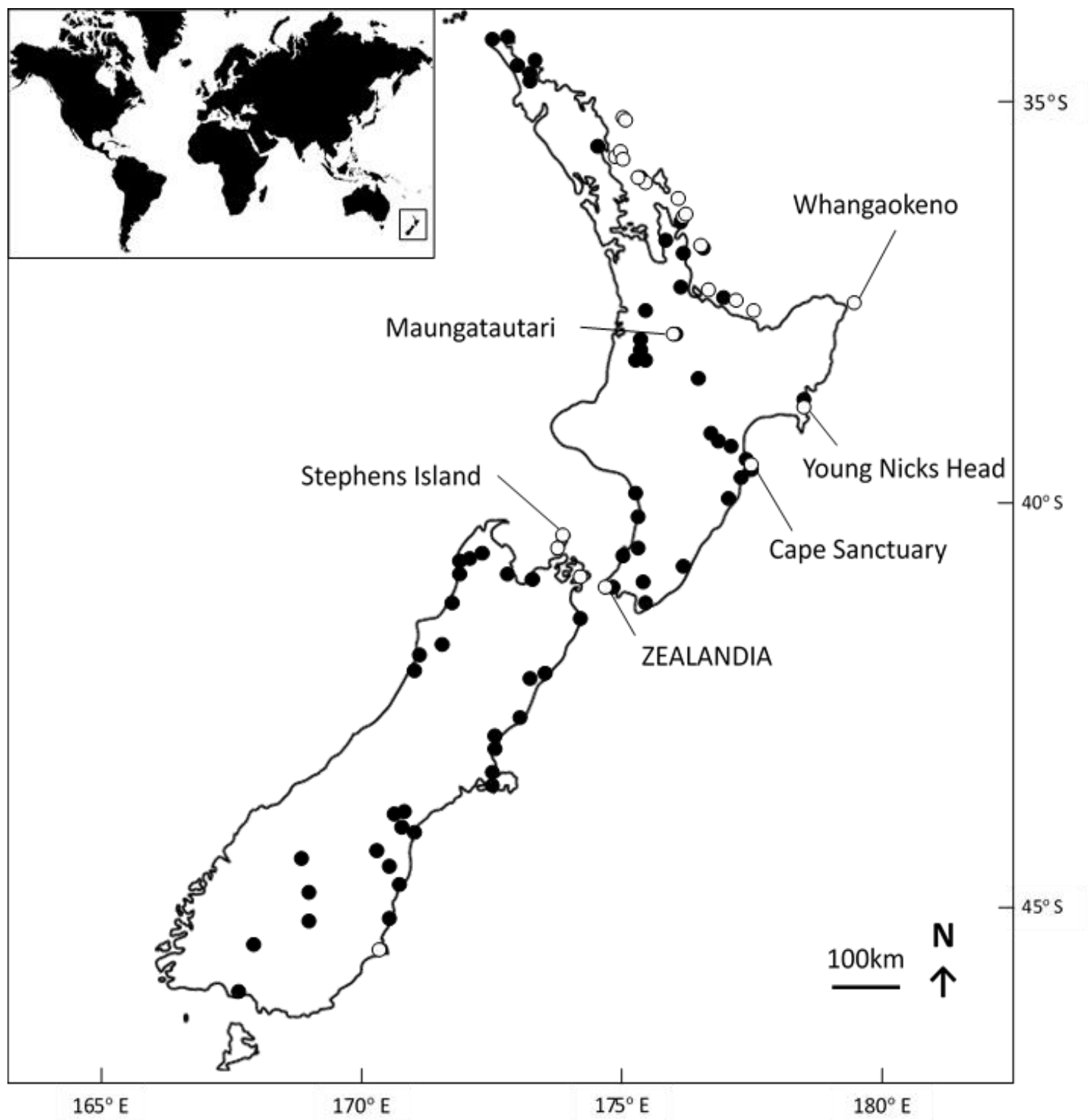

Figure 1.1 Map of the North and South Island, New Zealand showing locations where Holocene subfossil remains have been found (black circles) and island groups/areas in which tuatara populations, both translocated and naturally occurring, are currently present (white circles). The majority of existing populations are on offshore islands, though several mainland sanctuary sites (including translocation sites featured in this study) are indicated. Sites reported on in this study are labelled and include: the October 2012 translocation sites (Cape Sanctuary, Maungatautari, Young Nicks Head and Whangaokeno), the source site (Stephens Island) and comparison site (ZEALANDIA ${ }^{\mathrm{TM}}$ ). Map adapted from Miller et al. (2014). 
monitored by a research group at Otago University in Dunedin and several studies have been published on this population's progress (Jarvie et al. 2014, 2015, 2016). These were the first large-scale, long-distance, mainland (with the exception of Whangaokeno) translocations undertaken for this species and are a first small step in restoring tuatara to their former distribution on the New Zealand mainland. In order to address the initial cause of population decline (primarily invasive mammals), and because the mainland is far from pest-free, tuatara translocated to mainland sanctuaries are protected by both predator and pest-proof fences, within which mammalian predators have been eradicated and pest control is on-going. As each translocation site is latitudinally distant from the source site, the consequently diverse environmental conditions and different habitats offer the opportunity to study tuatara in a range of environments. The comparatively warmer climates at Cape Sanctuary, Young Nicks Head Sanctuary, and Whangaokeno, which are on average $2-4^{\circ} \mathrm{C}$ warmer during summer months, provide natural models for temporal climate change, enabling an assessment of the ability of tuatara to adjust to a warming climate. The broad active temperature range of tuatara means they can be considered a reasonably thermally robust species; however, there is a risk that local adaptation to the Stephens Island climate might influence their ability to establish successfully (Miller et al. 2012). Equally, local species may present other risks so short-term monitoring following release will be vital to evaluating the progress of these translocations as well as quickly identifying potential threats to the translocated tuatara (Miller et al. 2014).

The movement of tuatara from Stephens Island was part of a conservation translocation, involving intentional movement to benefit conservation goals (IUCN/SSC 2013). As tuatara were released at sites within their historical range these translocations could also be deemed reintroductions under the same IUCN guidelines (Seddon et al. 2012; IUCN/SSC 2013). However, considering that there is substantial genetic variation between island populations of tuatara, the likelihood that extant Stephens Island tuatara are genetically similar to their mainland-dwelling ancestors is unknown, therefore this movement of tuatara will be referred to as a conservation translocation (MacAvoy et al. 2007; Hay et al. 2010; Miller et al. 2012). 


\subsection{Bacteria: Commensal and potentially pathogenic microorganisms, Salmonella spp. and Campylobacter spp.}

The interaction between environmental temperature and disease is an important, but often overlooked, aspect of reptile conservation in the face of anthropogenic climate change. A principal concern regarding anthropogenic climate change is that reptiles may become more susceptible to disease as the climate warms owing to potentially increased pathogen growth rates, survival and transmission capabilities, as well as increased host susceptibility (Harvell et al. 2002). Warming temperatures have already been linked to an increase in reports of disease in marine fauna and temperature-dependent bacterial pathogenicity has been observed in Cnidarians (Romano et al. 2000; Ward \& Lafferty 2004; Bally \& Garrabou 2007). Similarly, infectious diseases responsible for global amphibian declines and extinctions may be aided by climate change (Daszak et al. 1999; Pounds et al. 2006). Understanding the impact of a shift in climate on reptile health is a high conservation priority, especially for cold-adapted reptiles like tuatara that are particularly vulnerable to increasing environmental temperatures. However, to date there has been little work on how climate change might impact the prevalence of both commensal and potentially pathogenic bacteria in such species.

Pathogenic bacteria like Salmonella spp. are a risk to wildlife as they can reduce individual fitness and population viability through impaired reproductive success, increased vulnerability to predation and large-scale mortalities (Anderson \& May 1979; Scott 1988; Janssens \& Stoks 2014). Salmonella spp. are enteric, facultatively anaerobic, gram-negative, pathogenic, and potentially fatal bacteria present in the environment and multiple animal taxa including mammals, birds and reptiles (Rabsch et al. 2015). While relatively common in reptiles worldwide (with population prevalence, the proportion of cases in a population, sometimes in excess of 90\%), a considerably lower prevalence of 0$10 \%$ has been found in New Zealand reptiles (Hoff \& White 1977; Otokunefor et al. 2003; Chambers \& Hulse 2006; Middleton et al. 2010; Franco et al. 2011; Bull et al. 2012; Baling et al. 2013a). Transmission is usually via faecally-contaminated food and water, soil or contact with infected organisms and as these bacteria are able to survive in water, soil and faeces for long periods of time outside of a host there are numerous routes and opportunities by which infection can occur (Mermin et al. 2004; Johnson-Delaney \& 
Mader 2006; Haraga et al. 2008; Broz et al. 2012; Bull et al. 2012). Infection alone is not a risk to an individual's health as many Salmonella spp. serovars (variations within the species of bacteria) are likely benign commensal organisms, part of the normal gut flora, and Salmonella spp. are regularly isolated from the intestinal tracts of clinically normal, asymptomatic reptiles (Chiodini \& Sundberg 1981; Bäumler et al. 1998; Mitchell \& Shane 2001; Barrow et al. 2011). Asymptomatic animals can be considered bacterial reservoirs that will intermittently shed the bacteria and facilitate transmission to uninfected individuals in which the bacteria could cause systemic illness. Numerous cases have been documented in which shedding by captive reptiles (pets or display animals) has been implicated as a potential route of infection and cause of Salmonellosis in humans (Mermin et al. 1997, 2004; Woodward et al. 1997; Friedman et al. 1998; Pfleger et al. 2003; Bauwens et al. 2006; Gray 2011; Middleton et al. 2014).

Post-ingestion, colonisation by Salmonella spp. is usually inhibited by thick epithelial mucus layers, antimicrobial peptides, and volatile organic acids produced by commensal organisms in the gut (Mahida et al. 1997; Broz et al. 2012). However, stressors such as heat, transport, overcrowding, captivity, resource shortage and illness can suppress the immune system and disrupt normal gut flora, allowing pathogenic bacteria to invade the intestinal epithelium and cause systemic disease (Cambre et al. 1980; Carey 1993; Alford \& Richards 1999; Gibbons et al. 2000; Pasmans et al. 2003; Franco et al. 2011). Salmonellosis, the diseased stage of Salmonella spp. infection, can present in reptiles with a variety of symptoms including reproductive failure and disease, abscessation, septicaemia, pneumonia, inflammation and death (Johnson-Delaney \& Mader 2006). An increase in prevalence in humans and birds in past decades (both within New Zealand and overseas) has resulted in its classification as an emerging infectious disease (Daszak et al. 2000; Dobson \& Foufopoulos 2001; van Andel et al. 2014). In New Zealand, Salmonella spp. have been identified in species of native and introduced reptiles, birds and mammals and have been implicated in the deaths of hihi (Notiomystis cincta), masked lapwing (Vanellus miles novaehollandiae), tuatara, saddleback (Philestumus carunculatus), New Zealand sea lions (Phocarctos hookeri) and livestock (McInnes 1971; Clark 2001; Alley et al. 2002; Clark et al. 2002; Castinel et al. 2007; Middleton et al. 2010, 2014; Kikillus et al. 2011; van Andel et al. 2014). 
Campylobacter spp., like Salmonella spp., are enteric, gram-negative bacteria and are able to survive temporarily outside of the host, enabling transmission via contaminated food, water and soil (Tu et al. 2004; Bull et al. 2006; Inglis et al. 2010; Jäderlund et al. 2010). Several species are considered to be emerging pathogens, with Campylobacteriosis considered a major public health problem in New Zealand (Mullner et al. 2009; Man 2011). Campylobacter spp. have been isolated from birds, mammals and reptiles and, while it can be pathogenic, is considered to be commensal in the intestinal tract of many species. It has been isolated from clinically normal reptiles, with no reports in the literature of Campylobacter spp. affecting disease in this taxa (Harvey \& Greenwood 1985; Man 2011; Wang et al. 2013; Giacomelli \& Piccirillo 2014; Gilbert et al. 2014). While the prevalence of Campylobacter spp. in wild birds, livestock and waterways in New Zealand has been widely studied due to their relevance as human pathogens, there is little in the literature regarding prevalence in native reptiles (Ikram et al. 1994; EberhartPhillips et al. 1997; Savill et al. 2001; French et al. 2009; Sears et al. 2011).

Despite close associations of tuatara with species known to excrete Salmonella spp. (i.e. seabirds and skinks) and its presence in soil around burrows, analysis of over 600 samples did not isolate Salmonella spp. from any live tuatara (Tizard 2004; Gartrell et al. 2007; Middleton et al. 2010, 2014). Equally, previous work to detect Campylobacter spp. in tuatara has been equivocal (Dr. B. Gartrell, Dr. A. Midwinter pers. comm.). Prevalence of Salmonella spp. in the environment and its long life span outside of hosts makes lack of exposure an unlikely explanation, but alternative hypotheses are conflicting, suggesting that tuatara could be innately resistant to Salmonella spp., or that the species' low body temperatures might inhibit bacterial proliferation (Johnson-Delaney \& Mader 2006; Gartrell et al. 2007; Middleton et al. 2014). The stress-associated intermittence with which bacteria are shed from the intestinal tract can also produce false negative results, making detection problematic (Gartrell et al. 2007).

Salmonella spp. have a growth temperature range of $5^{\circ} \mathrm{C}-45^{\circ} \mathrm{C}$, with optimal growth occurring between $35^{\circ} \mathrm{C}$ and $37^{\circ} \mathrm{C}$ (Tajkarimi 2007). Conversely, tuatara have an activity body temperature range of $5^{\circ} \mathrm{C}-30^{\circ} \mathrm{C}$, below the optimum temperature range for Salmonella spp. growth. As other cold-adapted New Zealand reptiles have been found to carry a range of Salmonella spp. serovars it is unlikely that suboptimal incubation temperatures alone would make tuatara resistant to Salmonella spp. colonisation 
(Middleton et al. 2010). The identification of Salmonella-responsive antibodies in tuatara suggests that that the inability to detect Salmonella spp. may be partially due to an adaptive immune response (Middleton et al. 2015). Supposing that the low body temperatures maintained on Stephens Island contribute to the inhibition of Salmonella spp. colonisation, then warmer environmental temperatures could enable tuatara to maintain higher body temperatures for longer periods of time, which could be more favourable to and enable bacterial proliferation. Increasing environmental temperatures under anthropogenic climate change are also pertinent to thermal-stress in cold-adapted ectotherms; if a pathogenic bacterium's thermal optimum exceeds that of its host, then warmer temperatures could impair the host's immune response while simultaneously improving conditions for the pathogen (Harvell et al. 2002). There are also disease risks inherent to translocation, with translocated animals not only at risk of exposure to novel species and pathogens, but also potential carriers of pathogens that they may introduce to naïve local populations (Jacobson 1993; Tompkins et al. 2011; Almberg et al. 2012). Similarly, translocation-associated stressors like capture, confinement, handling, transport and re-establishment in a new environment can also cause immunosuppression and cause individuals to be more susceptible to infection on release (Dickens et al. 2010; Kock et al. 2010).

\subsection{Parasites: ticks, mites, haemoparasites and intestinal parasites}

Parasites can negatively impact factors ranging from growth and behaviour to fecundity and survival, and pose a substantial threat to the conservation of their hosts (Scott 1988; Brown et al. 1995; Moller \& Nielsen 2007; Zamora-Vilchis et al. 2012; Reisinger et al. 2015). At the same time, parasites can contribute to the maintenance of genetic diversity, regulate host population size, mediate competition between host species and influence community composition, and can therefore be integral parts of ecosystems (Hudson et al. 1992; Hudson \& Greenman 1998; Windsor 1998; O’Brien 2000; Harvell et al. 2002; Godfrey et al. 2008). Climate plays a significant role in the extent to which a parasite is able to infect hosts through effects on both organisms and their interactions. Exposure of hosts to stressful abiotic conditions (e.g. suboptimal temperatures, drought) or events (e.g. pollution, predation, translocation) as well as seasonal fluctuations in hormone levels 
can cause immunosuppression. Poor individual fitness can also result in a lower immune response, which can facilitate increased parasite prevalence and infection intensity (Esch et al. 1975; Oppliger et al. 1998; Klukowski \& Nelson 2001; Cichoń et al. 2002; Navarro et al. 2003; De Kloet et al. 2005; Ujvari \& Madsen 2006; Cox \& John-Alder 2007; Teixeira et al. 2007). Consequently, increased air temperatures, adverse environmental conditions, and ecological disturbances predicted under anthropogenic climate change could facilitate increases in parasite abundance, which could be substantially detrimental to host populations (Brooks \& Hoberg 2007).

While adverse biotic and abiotic factors can increase host vulnerability to parasitism, the exposure of parasites to different thermal climates can also facilitate changes in parasite prevalence. Warmer temperatures can enhance biochemical activity, speed up growth and development rates, prompt reproduction and advance life cycle completion rates, whereas low temperatures can reduce parasite abundance through the inhibition of development and slowing of life cycles, processes which have been observed in species of tapeworm, parasitoid wasps, ticks and mites (Nealis et al. 1984; Perring et al. 1984; Oliver 1989; Chilton \& Bull 1991; Ogden et al. 2004; Godfrey et al. 2008; Lafferty 2009; MacNab \& Barber 2012; IPCC 2014). However, the maintenance of an increased temperature-dependent metabolic rate would also require higher resource consumption in parasites and could result in decreased survivorship if energetic demands were not met, which is a particular risk during free-living developmental stages when parasites are unable to feed on their hosts (King \& Monis 2007; Lafferty 2009). This relationship has been observed in the apicomplexan parasite Cryptosporidium parvum, which suffers deteriorating survival rates with continued exposure to warm temperatures (Fayer et al. 1998).

Equally, warmer temperatures could disturb seasonal reproductive cycles, disrupt host-parasite synchronies and, if rising temperatures exceed those optimal for parasite growth and transmission, climate change also has the potential to bring about a decrease in parasite prevalence, though it is possible that such temperatures would also be detrimental to a cold-adapted host and so both parties would likely suffer (Polley \& Thompson 2009; Karvonen et al. 2010). Environmental temperature is especially influential to parasites of ectotherms like tuatara, whose body temperature and physiological function is strongly affected by the immediate thermal environment, 
offering parasites little protection against changing climatic conditions (Deutsch et al. 2008; Polley \& Thompson 2009).

Anthropogenic climate change is a significant conservation concern to wildlife around the world and while conservation translocations may help mitigate some of the risk to vulnerable species there are translocation-associated risks that can be difficult to avoid (IUCN/SSC 2013; IPCC 2014). Although disease screening is often implemented to prevent the transmission of novel pathogens and parasites into naïve local populations, translocated animals are vulnerable to spillover from local reservoirs and are generally expected to acquire parasites and pathogens post-release (Leighton 2002; Gartrell et al. 2006, 2007; Germano \& Bishop 2008; Kock et al. 2010; Almberg et al. 2012). As increased disease prevalence has been linked to translocation failure and parasites themselves can be pathogenic or act as vectors for other pathogens, it is important that parasite status is monitored post-release so that potential threats to the translocated population can be quickly detected and addressed where appropriate (Cunningham 1996; Daszak et al. 1999; de la Fuente et al. 2008). Equally, as many species of parasite are host-specific they can be as vulnerable and important to biodiversity as their hosts, and there may be an ethical obligation to ensure that parasite assemblages are maintained following translocation (Daszak et al. 2000).

The tuatara is host to a intraerythrocytic apicomplexan haemoparasite (Hepatozoon tuatarae), its proposed arachnid ectoparasite vector, the tuatara tick (Amblyomma sphenodonti), and two species of trombiculid mite (Neotrombicula sphenodonti and Neotrombicula naultini; Dumbleton 1943; Goff et al. 1987; Godfrey et al. 2010a). Presence of coccidial oocysts, nematode ova, and motile protozoa have also been documented in their faeces (Gartrell et al. 2006). Both H. tuatarae and A. sphenodonti are host-specific parasites, feeding only on tuatara, whilst mites (Neotrombicula spp.) have also been observed on other New Zealand reptiles like the common skink (Oligosoma polychroma) and common gecko (Woodworthia maculata) on Stephens Island (Goff et al. 1987; Godfrey et al. 2008, 2010a). Infestation with ticks and mites has been shown to reduce body condition in tuatara; however, no correlation between $H$. tuatarae and body condition has been found and there is low overall prevalence and infection intensity in the most studied tuatara population on Stephens Island (17-19\% prevalence, $<0.01-0.1 \%$ of blood cells infected; Godfrey et al. 2010a, 2011a). 
Amblyomma sphenodonti was originally classified as belonging to the genus Aponomma, but was transferred to Amblyomma following a taxonomic revision in 2002 (Klompen et al. 2002). The Amblyomma genus includes around 140 different tick species, which infect a wide range of hosts from birds and reptiles to mammals (including humans), not all of which are host-specific throughout their life stages (Garris 1984; Allan et al. 1998; Labruna et al. 2002, 2003, 2004; Pinter et al. 2004; Levin 2015). Of the 140 known Amblyomma species, around 37 are found to parasitize reptiles; however, phylogenetic analysis has shown that $A$. sphenodonti are not closely related to other Amblyomma ticks and it has been suggested that a separate genus might be justified (Miller et al. 2007; Levin 2015). The tuatara tick is considered endangered and relict and has a more severely restricted distribution than even its range-restricted host, occurring on only eight of 28 tuatara-occupied offshore islands on which tuatara were sampled in 1988-92 and 2005 (Durden \& Keirans 1996; Miller et al. 2007; Buckley et al. 2012). A lack of gene flow between island populations and low genetic diversity within populations, as well as similar genetic patterns between $A$. sphenodonti and tuatara also suggests that the tick populations have likely been affected by extended tuatara population bottlenecks and possible co-evolution between the two species (Miller et al. 2007).

Amblyomma sphenodonti has a three-host lifecycle and feeds on tuatara during each developmental stage (larvae, nymph and adult; Heath 2006). Female ticks detach once engorged to moult into the next stage or to lay eggs, whereas male ticks do not engorge, instead remaining on the host to mate (Dumbleton 1943; Heath 2006; Godfrey et al. 2008). Transmission of $A$. sphenodonti to their hosts requires direct contact, so host and parasite must occupy the same environment for attachment to occur (Godfrey et al. 2011b). Unfed larval ticks have been found to survive between 177 and 248 days off host whereas adult $A$. sphenodonti have been shown to survive for even longer periods (males: up to 582 days; females: up to 405 days) when maintained in constant temperature and high humidity laboratory conditions $\left(18^{\circ} \mathrm{C}, 90-95 \%\right.$; Heath 2006$)$. However, as maintaining body-water equilibrium is key to off-host survival, desiccation, as well as predation and eventual starvation pose significant risks during free-living periods, especially if reattachment does not occur (Bull et al. 1988; Oliver 1989; Needham \& Teel 1991). As such, ticks are considered unlikely to travel far from sites where they detach and $A$. sphenodonti is thought to employ a "sit-and-wait" strategy seen in related tick species, 
residing in refuges frequented by tuatara (Petney et al. 1983; Duffield \& Bull 1996; Kerr \& Bull 2006; Godfrey et al. 2010a, 2011b). Accordingly, laboratory microcosm and activity experiments have found that $A$. sphenodonti, like their hosts, are most active at night and show microhabitat preferences for moist, coarse, and shaded substrates, comparable to the inside of a tuatara burrow, thereby reducing desiccation risk and maximising reattachment opportunities (Heath 2006; Kerr \& Bull 2006; Godfrey et al. 2011b).

Like ticks, transmission of trombiculid mites is also reliant on contact with hosts, though $N$. naultini and $N$. sphenodonti only attach to tuatara in the larval stage, which requires tuatara to interact with locations where female mites laid eggs (Godfrey et al. 2010b). When infecting tuatara the larvae tend to cluster in areas where they are least exposed to intense abiotic conditions or likely to be brushed off during movement, such as the neck and base of the hind legs (Arnold 1986; Godfrey et al. 2010a). Unlike ticks, which feed on the blood of their hosts, larval mites or "chiggers" feed on cellular debris and tissue fluid that is broken down by the mites saliva (Arnold 1986). The nymph and adult stages, which are not parasitic on tuatara, are thought to be predatory, feeding on other invertebrates in the environment (Wharton \& Fuller 1952; Sasa 1961).

Both ticks and mites have also been implicated as potential vectors of $H$. tuatarae, though a lack of haemoparasite developmental stages within mites and positive correlations between $A$. sphenodonti abundance and $H$. tuatarae infection intensity implicates ticks as the likely vectors, meaning that a reduced tick prevalence could affect a reduction in haemoparasite prevalence (Godfrey et al. 2011a). The correlation between tick and haemoparasite loads could be attributed to an impaired immune response associated with high endoparasite loads, an overall impaired immune response increasing vulnerability to both parasites, or tuatara with higher tick loads might simply be more likely to ingest infected ticks (Godfrey et al. 2011a). Little is known about the biology of H. tuatarae; however, if its life cycle is comparable to that of other Hepatozoon species it is likely that $H$. tuatarae infect ticks to reproduce and the identification of developmental stages within A. sphenodonti supports this possibility (Smith 1996; Herbert et al. 2010; Godfrey et al. 2011a). The lifecycles of related Hepatozoon species rely on ingestion of the invertebrate hosts (e.g. tick) by an intermediate vertebrate host (i.e. a tuatara, substantiated by the presence of $A$. sphenodonti remains in scat), allowing the haemoparasite to develop within the intermediate vertebrate host's internal organs 
before producing merozoites that infect host red blood cells. These red blood cells are then consumed by feeding ticks, and the transmission cycle begins anew (Walls 1981; Smith 1996; Godfrey et al. 2011a).

It has been established that there is seasonal variation in abundance of the ectoparasites A. sphenodonti and Neotrombicula spp. (Godfrey et al. 2008). Tick abundance on Stephens Island tuatara has been found to be lowest in the summer months, with peaks from late autumn through to early spring, whereas mite loads are highest in summer and autumn and peak in March (early autumn), but are not found on tuatara throughout the rest of the year (Godfrey et al. 2008). Conversely, haemoparasite prevalence and intensity is generally low on Stephens Island and temporally stable (Godfrey et al. 2011a). A. sphenodonti abundance contrasts with abundance results from a related reptile tick, Amblyomma limbatum, which has a high attachment rate to its lizard host Tiliqua rugosa when ambient temperature is high (Kerr \& Bull 2006). The explanation for this likely lies in the different refuge-seeking behaviours of the two hosts; T. rugosa shelter in burrows, sometimes for days, when ambient temperatures are high whilst tuatara demonstrate periods of reduced activity when temperatures are low, spending long periods of time in burrows over winter; therefore, periods of maximum exposure to refuge-dwelling ticks differ seasonally (Walls 1983; Seebacher 2005; Kerr \& Bull 2006). Similar patterns of winter peaks and summer troughs have been observed in the tick Aponomma gervaisi, which parasitises monitor lizards (Varanus spp.). These reptiles also shelter in burrows during the colder winter months and this increase in abundance has been attributed to increased exposure to $A$. gervaisi in the burrow (Auffenberg \& Auffenberg 1990).

It is expected that local and translocated wildlife will come into contact with new parasites and pathogens following translocation and there is potential for translocationassociated stressors (e.g. capture, examination, transport, release into a new environment) to elevate glucocorticoid levels, the prolonged elevation of which can reduce host immunocompetence and increase the susceptibility of tuatara to infection (Teixeira et al. 2007; Godfrey et al. 2008; Dickens et al. 2010). While the tuatara-specific nature of $A$. sphenodonti and $H$. tuatarae reduces the risk of transmission between tuatara and local lizards at release sites, the same cannot be said of trombiculid mites, the larvae of which can parasitise a wide range of hosts from multiple taxa, with high 
abundance negatively associated with host reproduction, growth rate and survival (Klukowski \& Nelson 2001; Curtis \& Baird 2008; Lin et al. 2014). For instance, the mite Trombicula alfreddugesi has been observed on 52 avian, 39 reptilian, 32 mammalian and three amphibian species while $N$. sphenodonti and $N$. naultini have also been observed on other New Zealand reptiles, though prevalences in lizard populations at the translocation sites monitored in this study have not been determined (Wharton \& Fuller 1952; Arnold 1986; Benton 1987; Godfrey et al. 2008, 2010a). This lack of host-specificity contributes to the risk of trombiculid mites as vectors of disease. The mites' feeding action can also cause direct damage to the host's skin, leaving the resulting wound vulnerable to secondary infection (Arnold 1986).

Tuatara may experience reduced parasite loads in the short term following translocation. McKenzie (2007) documented significant declines in both tick and mite populations following translocation of tuatara from Stephens Island to ZEALANDIA ${ }^{\mathrm{TM}}$, a mainland sanctuary in New Zealand. Ticks were also found to persist at very low abundances following the translocation of tuatara to Tiritiri Matangi Island (Moir et al. 2012). Parasite transmission is generally density-dependent and some of the sites to which tuatara were translocated in 2012 have substantially lower population densities than the source site, which may enable tuatara to maintain larger home ranges or disperse further than tuatara resident on Stephens Island (McKenzie 2007; Almberg et al. 2012). Ticks translocated with their hosts may therefore detach in a burrow that is not revisited or visited as frequently, which could reduce overall tick load and reattachment opportunities, and larger home ranges or a decrease in territory overlap with neighbouring tuatara could reduce transmission opportunities (McKenzie 2007; Godfrey et al. 2010b). As such, the interactive effect of translocation and climate change could influence tuatara and parasite interactions both positively and negatively and studies of translocated populations are required to determine the potential impact on these communities.

\subsection{Thermoregulation and nocturnal activity}

The optimal temperatures and thermal sensitivities of enzymes, cell membranes and aerobic respiration results in thermal boundaries for biological reactions from the cell to 
organismal level, beyond which an organism will cease to function normally (Angilletta 2009). Consequently, an organism's biological functions (i.e. growth, feeding, reproduction, locomotion) are dependent on the maintenance of suitable body temperatures (Angilletta 2009). Endothermic taxa like mammals and birds are able to maintain thermal homeostasis through metabolic processes, which helps to buffer them against suboptimal environmental temperatures (Lowell \& Spiegelman 2000; Boyles et al. 2011). In contrast, reptiles and other ectothermic taxa, which obtain a comparatively small amount of heat from internal sources, are dependent upon suitable environmental temperatures to effectively regulate body temperature and maintain normal physiological function, making them more sensitive to changes in environmental temperature (Deutsch et al. 2008).

The rate at which anthropogenic climate change is advancing is a significant conservation concern and associated environmental impacts are expected to have substantial effects on biodiversity (Parmesan 2006; IPCC 2014). Ectothermic taxa, like reptiles, may be especially affected by the rising air temperatures because their physiological and ecological performance is so dependent on environmental temperature (Huey \& Kingsolver 1989). While predictions of costs and benefits to ectothermic taxa vary, the actual impact of rising temperature will likely depend on the physiological sensitivities of individual organisms to changes in air temperature, which are often reflective of the variation in temperatures they endure, and as air temperatures continue to rise it is likely that environmental temperatures will approach or surpass the thermal tolerance limits of even the more robust species (Addo-Bediako et al. 2000; Calosi et al. 2008; Deutsch et al. 2008; Hoffman \& Sgro 2011; Hoffman et al. 2013). In order to survive organisms might mitigate the dangers associated with temperature increases (i.e. extirpation/extinction) through dispersal, adaptation, acclimation or behavioural plasticity (e.g. of thermoregulatory behaviour; Deutsch et al. 2008; Kearney et al. 2009; Hoffman \& Sgro 2011; Quintero \& Wiens 2013).

In order to regulate body temperature reptiles typically employ a suite of behavioural strategies that enable effective exploitation of the thermal environment. These behaviours include: 1 ) selection of suitable microhabitats (e.g. movement between sun and shade); 2) restriction of activity (e.g. periods of inactivity when temperatures are unsuitable); and 3) changes in posture and body shape (e.g. flattening of the body to 
increase surface area, orientation towards a heat source; Huey \& Pianka 1977; Huey 1982; Stevenson 1985a; Losos 1987; Kearney \& Predavec 2000). Although the thermal environment can be substantially heterogeneous, some reptiles are capable of precise thermoregulation, keeping their body temperature within a narrow optimal range, while others are considered thermoconformers, active across a broad range of body temperatures (Kearney \& Predavec 2000; Besson \& Cree 2010). Although behavioural mechanisms can be effective, an organism's ability to maintain its preferred body temperature is ultimately dependent upon the thermal quality of the environment, which is influenced by factors like solar radiation, humidity and habitat structure (e.g. availability of shade and sun; Huey 1974; Saint Girons 1980; Besson \& Cree 2010; Boyles et al. 2011). Reptiles in poorer quality environments (those with few thermally favourable sites) have been found to spend more time and energy on thermoregulation, which can reduce the amount of time available for other beneficial behaviours like mating, foraging, territory defence and predator avoidance (Huey 1974, 1982; Huey \& Slatkin 1976; Seebacher 1999).

Tuatara are considered to be a primarily nocturnal and cold-adapted species, though they have often been observed to bask close to burrow entrances during the day and may actually be more diurno-nocturnal than exclusively nocturnal (Werner \& Whitaker 1978). Because they forage at low overnight temperatures diurnal basking is thought to be an important way in which nocturnal foragers can support digestion (Werner \& Whitaker 1978). The tuatara's cold adapted lifestyle, combined with limited dispersal ability from offshore islands, places tuatara at particular risk from rising air temperatures, while also making them an ideal species for studying the early effects of climate warming (Saint Girons et al. 1980; Thompson \& Daugherty 1998; Blanchard 2002; Nelson et al. 2002b; Mitchell et al. 2008; Besson \& Cree 2010; Corkery et al. 2014a).

The accuracy with which tuatara regulate body temperature has been debated in the past, with some suggesting that they actively thermoregulate whereas others have found limited evidence of this, proposing that the species is more thermoconformatory, with mean body temperatures of Stephens Island tuatara found to match or fall within $1^{\circ} \mathrm{C}$ of mean environmental temperatures (Saint Girons 1980; Saint Girons et al. 1980; Barwick 1982; Walls 1983; Thompson \& Daugherty 1998; Corkery 2012; Cree 2014). However, plasticity of thermoregulatory behaviour has been observed, with tuatara 
found to modulate their thermoregulatory effectiveness in response to quality of the thermal environment, achieving higher body temperatures and thermoregulating more precisely when in low-quality habitat (i.e. with limited basking opportunities; Besson \& Cree 2010). Tuatara thermoregulatory behaviour also appears to be influenced by habitat structure, with tuatara rarely observed basking in exposed areas (e.g. grassland/pasture), but frequently seen out of burrows in forested habitat or within protected retreats penetrated by solar radiation when no canopy is present (Werner \& Whitaker 1978; Saint Girons 1980; Gans 1983; Gillingham \& Miller 1991). Apparent avoidance of open areas could be a response to habitat-influenced microclimates, or, considering that tuatara evolved in the presence of primarily avian predators, electing to bask under the cover of a closed canopy/shelter could be an anti-predatory behaviour (Huey 1991; Worthy \& Holdaway 2002; Cree 2014).

Recent translocations of tuatara from Stephens Island in the Cook Strait to four sites around New Zealand's North Island, several of which are on average $2-4^{\circ} \mathrm{C}$ warmer during summer months, provide surrogates for temporal climate change and enable an assessment of how tuatara might behaviourally adjust to a warming climate. Tuatara are capable of year round activity, though this is dependent on environmental conditions and they are known to enter periods of inactivity when temperatures are low, generally remaining in their burrows over winter but emerging when air temperatures and weather conditions allow (Walls 1983; Thompson \& Daugherty 1998; Seebacher 2005). As tuatara have been found to emerge earlier from burrows at warmer air temperatures it is possible that movement to sites with warmer climates (or rising air temperatures at currently cooler sites) could lift this environmental constraint and enable more frequent, prolonged periods of activity on a daily and annual timescale (Hughes 1967). Similarly, as thermoregulation is more costly in cooler climates, movement to an environment of higher thermal quality could see a reduction in time and energy spent thermoregulating (i.e. shuttling between warm and cool microclimates), allowing more time for other fitness-related activities (Huey 1974, 1982; Huey \& Slatkin 1976; Seebacher 1999; Besson \& Cree 2010). Other benefits of increased environmental temperatures may include enhanced locomotor function, which could improve anti-predator response, foraging ability and territory defence, and increased lifetime reproductive output due to comparatively earlier sexual maturity owing to enhanced temperature-dependent growth 
rates (Christian \& Tracy 1981; Avery et al. 1982; Castanet et al. 1988; Autumn \& De Nardo 1995; Besson \& Cree 2011).

Conversely, lower relative humidity levels and higher air temperatures at some translocation sites, which are predicted to intensify under climate change, could increase evaporative water loss from the skin (Hill 1982; Hennessy et al. 2007). This could not only lower body temperature and influence thermoregulatory behaviour, but as tuatara lose a substantial amount of water through their skin and water is required to maintain body weight, it could also constrain the amount of time that can be spent outside of the burrow, reducing overall activity and negatively impacting fitness-related behaviours (Bogert 1949; Hill 1982; Barwick 1982; Corkery et al. 2014a). The importance of evaporative water loss as a constraining factor could be intensified at sites that are drought-prone or have no natural water source (e.g. Cape Sanctuary, Young Nicks Head and Whangaokeno). As warmer, drier climates could have significant effects on tuatara survival it is important that site-specific limitations, potential consequences of these limitations and the ability of tuatara to appropriately modify their behaviour is assessed.

\subsection{Learning ability}

The ability of an organism to modify aspects of its physiology in response to the environment can be highly adaptive and ultimately increase fitness under variable or unpredictable conditions (Whitman \& Agrawal 2009). Plasticity in response to environmental change has been studied in reptiles and other taxa across a wide variety of traits including digestion, locomotion, morphology, life history, and behaviour (Lemons \& Crawshaw 1985; Seigel \& Ford 1991; Piersma \& Lindström 1997; Applebaum \& Heifetz 1999; Sabat \& Bozinovic 2000; McWilliams \& Karasov 2001; Johnston \& Temple 2002; Réale et al. 2003; Cardozo \& Chiaraviglio 2008; Clark et al. 2008; Aubret \& Shine 2009; Cullen et al. 2010; Clusella-Trullas et al. 2010). However, the plasticity of higher cognitive ability, specifically learning ability, has received comparatively little attention.

Learning is a relatively permanent change in behaviour as a direct result of experience (Shettleworth 2010). It is neurobiologically characterized by an increase in dendritic spine density and the formation/remodeling of excitatory synapses in corresponding brain regions (Moser et al. 1994; Geinisman 2000; McAllister 2000). The 
ability to learn is an ecological necessity and vital to behaviours like foraging, antipredator response, territoriality, communication and reproduction (Clayton 1990; Marcellini \& Jenssen 1991; Marler 1997; Laland \& Williams 1997; Freeberg 1999; Cristol \& Switzer 1999; Grieco et al. 2002; Griffin \& Evans 2003; Paulissen 2008; Noble et al. 2012, 2014; LaDage et al. 2012; Leal \& Powell 2012; Clark et al. 2013). A range of learning processes have been studied across multiple taxa, from vocal learning in grey parrots (Psittacus erithacus) and tool-use in Japanese macaques (Macaca fuscata), to associative learning in nematodes (Caenohabditis elegans) and spatial learning in three-spined sticklebacks (Gasterosteus aculeatus; Pepperberg et al. 2000; Odling-Smee \& Braithwaite 2003; Qin \& Wheeler 2007; Quallo et al. 2009).

Vertebrate taxa may have similarities in cognitive abilities and underlying neural mechanisms due to shared ancestry of this fundamental ability (Wilkinson \& Huber 2012). However, the reptilian lineage has been evolving independently for approximately $\mathbf{2 8 0}$ million years or more, which is ample time for evolutionary constraints to produce distinct dissimilarities in the learning process, both between vertebrate classes and extant reptilian orders (Macphail 1982; Wilkinson \& Huber 2012). Modern reptiles, the Squamata, Chelonia, Crocodilia and Rhynchocephalia, were once thought to be "stimulusbound", behaviourally deficient and capable of minimal learning (Burghardt 1977). However, this conclusion may have resulted from poor methodologies and a lack of ecologically valid stimuli in some early (pre-1970) studies (Burghardt 1977; Paulissen 2008). The first recorded experiment to assess reptile learning was conducted by Robert Yerkes in 1901, which investigated maze learning in a turtle (Clemmys guttata), followed by a 30-year pause before the publication of a similar study by Otto Tinklepaugh in 1932 (Yerkes 1901; Tinklepaugh 1932; Burghardt 1977; Gaalema 2011). This paucity has continued, with much of the learning literature being dominated by studies on mammals and birds, with Burghardt (1977) describing the literature on reptile cognition at the time as "scattered and frequently unavailable" (Wilkinson \& Huber 2012; Burghardt 2013). Though advances have since been made in this field, namely in the areas of spatial, visual and social cognition and the study of novel behaviour, progress is still slight when compared to the volume of research addressing cognition in other vertebrate taxa (Wilkinson \& Huber 2012). 
Reptiles have been shown to perform successfully across a range of learning paradigms, including instrumental conditioning, classical conditioning, habituation, discrimination training, associative learning, aversion learning, spatial learning and reversal learning (Burghardt 1977, 2013; Macphail 1982; Schall 2000; Paradis \& Cabanac 2004; Woo et al. 2009; Rodríguez-Prieto et al. 2010; Gaalema 2011; Wilkinson \& Huber 2012; Clark et al. 2013). More recently, social learning has been demonstrated within the Chelonia and Squamata, including the non-social tortoise Geochelone carbonaria, and the ability to solve novel motor tasks has been established in several lizard species (Manrod et al. 2008; Wilkinson et al. 2010; Davis \& Burghardt 2011; Leal \& Powell 2012; Clark et al. 2013; Noble et al. 2014; Kis et al. 2015). There is now a body of evidence suggesting that the "stimulus-bound" reptiles are actually capable of cognitive abilities and behavioural complexity comparable to that seen in mammals and birds (Kirkish et al. 1979; López et al. 2001; Manrod et al. 2008; LaDage et al. 2012; Wilkinson \& Huber 2012; Leal \& Powell 2012; Burghardt 2013).

Spatial learning in particular is considered to be an invaluable aspect of reptile cognition, which may explain why, beginning with the study by Yerkes (1901), the majority of cognitive research has focused on spatial learning abilities (Burghardt 1977; Wilkinson \& Huber 2012; Carazo et al. 2014). The capacity to quickly and flexibly learn to navigate to nest sites, territorial boundaries, refuges, potential prey and other important resources means that spatial learning underpins behaviours such as predator avoidance, foraging, reproduction and territoriality, which in turn influences individual fitness and survival (Noble et al. 2012; Burghardt 2013; Carazo et al. 2014). That many reptile species (with the exception of some crocodilians) lack parental care and may consequently have limited opportunities for conspecific interactions that could facilitate social learning also suggests that they should be capable of spatial learning (Garrick et al. 1978; Paulissen 2008; Vitt \& Caldwell 2009).

The hippocampal formation has been identified as crucial for spatial navigation and memory in birds and mammals. In reptiles the medial and dorsal cortices are considered to be homologues of this brain region and serve a similar function. Both hippocampal and medial/dorsal cortex lesions have been shown to impair aspects of spatial learning and memory. Similarly, species that occupy navigationally demanding ecological niches have been found to possess enlarged hippocampi and medial/dorsal 
cortices (Morris et al. 1982; Sutherland et al. 1982; Macphail 1982; Sherry \& Vaccarino 1989; Gaulin 1992; Bingman 1992; Sherry et al. 1993; Moser et al. 1994; Fremouw et al. 1997; Day et al. 1999a, 2001; Rodriguez et al. 2002; López et al. 2003; Roth et al. 2006; Maguire et al. 2006; LaDage et al. 2009; Holding et al. 2012).

Reptiles are able to utilise a variety of strategies for spatial navigation in an environment. Multiple studies have found evidence for the role of chemical, magnetic, solar, auditory and vibrational cues in navigation (Lenhardt 1981; Rodda 1984; Adler \& Phillips 1985; Owens et al. 1986; Hetherington 1989; Lawson \& Secoy 1991; Lohmann \& Lohmann 1996; Nichols et al. 2000; Russell et al. 2005). Holtzman et al. (1999) did not explicitly test orientation mechanisms but suggested that corn snakes (Elaphe guttata guttata) might have successfully navigated a Barnes maze using strategies ranging from visual cues to tactile cues to path integration. Similarly, Stone et al. (2000) were unable to identify a single consistently used cue and so proposed that juvenile spotted pythons (Anteresia maculosus) may have used tape on the arena floor as a tactile cue, as well as intra and extra-arena visual cues. The side-blotched lizard, Uta stansburiana, and little brown skink, Scincella lateralis, were also found to navigate to hides using distal visual cues and Day et al. (2003) found that whiptail lizards (Cnemidophorus inornatus) could navigate to an accessible hide using both position and visual cues, though were faster when using position cues (Paulissen 2008; LaDage et al. 2012). Conversely, Day et al. (1999) found that two species of Acanthodactylus lizard showed variability in the strategies used to solve a spatial task, but did not utilise the distal cues provided. Other studies on the turtle, Trachemys (Pseudemys) scripta, found evidence for the use of cognitive maps, in which mental representations of multiple distal cues enable the location of a goal using its position relative to these cues, which could be considered a highly adaptive strategy as it allows for the removal of some cues without affecting navigation (López et al. 2000, 2001, 2003; Wilkinson \& Huber 2012). Taken together, these studies demonstrate the wide variety of strategies employed by reptiles when navigating in an environment.

Ectothermic taxa like reptiles, fish, amphibians and invertebrates are dependent upon suitable environmental temperatures to effectively regulate body temperature and maintain normal physiological function (Deutsch et al. 2008; Hare \& Cree 2010). Several recent studies have demonstrated that incubation temperature can influence learning 
ability in some ectotherms, with "hot" incubated three-lined skinks (Bassiana duperreyi) found to be better learners than their "cold" incubated conspecifics in both spatial tasks and novel motor tasks (Amiel \& Shine 2012; Clark et al. 2013; Amiel et al. 2014). Similarly, honey bees (Apis mellifera) incubated at $35^{\circ} \mathrm{C}$ and $36^{\circ} \mathrm{C}$ displayed better short-term learning and memory than those reared at $32^{\circ} \mathrm{C}$ and $33^{\circ} \mathrm{C}$, though no effect on long-term memory was observed (Jones et al. 2005). Amiel and Shine (2012) suggest that incubation temperature may affect learning ability in hatchling skinks $B$. duperreyi through the modification of hormone levels or influence on endocrine pathways that consequently affect brain development, including regions like the medial and dorsal cortices, which control behaviours like learning in reptiles (Ahmad \& Zemenhof 1978; Day et al. 2001; Rissman et al. 2002; López et al. 2003; Valenzuela \& Lance 2004; Amiel \& Shine 2012). The exact mechanisms through which the thermal environment during embryogenesis affects neurogenesis and learning ability in reptiles are unknown, but in humans and other animals, including reptiles, there is evidence of temperature having direct action on neural cell development, and affecting cognition through epigenetic modifiers (Penafiel et al. 2001; Radmilovich et al. 2003; Wang et al. 2012; Lin et al. 2014; Torson et al. 2015; Maynard et al. 2016). The authors postulate that the enhanced learning ability of warmerincubated hatchlings may facilitate a more successful response to novel environmental change through improved behavioural flexibility (Amiel \& Shine 2012). The ability to respond quickly and effectively to novel change is of particular relevance in the face of increasing air temperatures and extreme weather predicted under anthropogenic climate change, which could generate novel changes to habitats and challenge local species (Amiel \& Shine 2012; IPCC 2014; Reisinger et al. 2014). Whilst the plasticity of learning in response to incubation temperature has been investigated in recent years, the plasticity of learning in response to ambient temperature during learning has not been well established.

The majority of research on the relationship between cognitive ability and ambient temperature has focused on mammalian, specifically human, cognition and the effect of heat stress (Blockley \& Lyman 1950; Chiles 1958; Mackworth 1961; Wing 1965; Grether 1973; Ramsey et al. 1975; Allen \& Fischer 1978; Hancock 1981, 1982; Ramsey \& Kwon 1992; Hancock \& Vasmatzidis 2003). A study on humans (Homo sapiens sapiens) by Allen and Fischer (1978) found that recall of a paired word-number list was optimal at $22^{\circ} \mathrm{C}$, but 
declined at higher and lower temperatures $\left(11,17,28\right.$ and $\left.33^{\circ} \mathrm{C}\right)$. Similarly, numerous studies have addressed the association between cognitive ability and heat stress in humans, with most studies reporting decrements in cognitive performance as environmental temperature exceeds $29^{\circ} \mathrm{C}$ (Blockley \& Lyman 1950; Chiles 1958; Mackworth 1961; Wing 1965; Grether 1973; Ramsey et al. 1975; Hancock 1981, 1982; Ramsey \& Kwon 1992; Hancock \& Vasmatzidis 2003). Temperature has also been found to influence learning in fluoride toxicated rats, with acquisition and retention of a memory task shown to be poorer after exercise in high and low $\left(20^{\circ} \mathrm{C}\right.$ and $\left.35^{\circ} \mathrm{C}\right)$ regimes when compared to thermo-neutral treatments $\left(25^{\circ} \mathrm{C}\right.$ and $30^{\circ} \mathrm{C}$; Basha \& Sujitha 2012). Despite this bias, there have been several studies using ectothermic taxa that have found an effect of ambient temperature on learning ability. Goldfish (Carassius auratus) and carp (Cyprinus carpio) were found to demonstrate temperature-dependent learning and retention on maze, swimming, and avoidance tasks (French 1942; Andry et al. 1971; Zerbolio 1973; Borsook et al. 1978). Similarly, an experiment by Krekorian et al. (1968) on desert iguanas (Dipsosaurus dorsalis) found that animals trained at the lowest temperature $\left(22^{\circ} \mathrm{C}\right)$ were unable to solve a maze task whilst those trained at the highest temperature $\left(32^{\circ} \mathrm{C}\right)$ were the most successful learners. Body temperature has also been found to influence neural activity in garter snakes (Thamnopsis radix and $T$. sirtalis), which the authors speculate might be important to learning ability (Andry et al. 1971).

Within the Reptilia, the majority of cognitive studies have focused on the Chelonia, though studies on squamates and crocodilians have shown evidence of reptilian capability in most traditional learning paradigms (Burghardt 2013). However, I am aware of only two studies in the literature that have investigated learning in the tuatara (Northcutt \& Heath 1973; Woo et al. 2009). One, by Northcutt and Heath (1973), studied the performance of two adults (a male and female) on a T-maze spatial task in which both animals were required to reverse their natural turning preferences in order to access their home pen (reward). The authors found that both animals reached criterion ( $<20 \%$ error) in trial block four or five (trials 15-25) and showed comparable learning rates to caimans (Caiman sclerops) run on the same task (Northcutt \& Heath 1971, 1973). In the second study, Woo et al. (2009) trained juvenile tuatara on an operant learning task in order to examine visual sensitivity. All 17 animals were successfully trained to discriminate between two stimuli (constant vs. oscillating LED lights) and associate the oscillating light with a food reward. 
Successful learning of the association then enabled the authors to manipulate the rewarded stimulus and investigate the ability of tuatara to perceive different frequencies of flickering light (Woo et al. 2009). Similar abilities to associate visual cues with a reward have been observed in a range of reptiles (e.g. (Day et al. 1999b, 2003; López et al. 2000, 2003; Gaalema 2011; Leal \& Powell 2012).

The ability to respond quickly and effectively to novel change is of particular relevance in the face of anthropogenic climate change, which could generate novel changes to habitats and challenge the survival of local species (Williams et al. 2007; Amiel \& Shine 2012; IPCC 2014; Reisinger et al. 2014). Due to their extreme longevity ( 100 years; Dawbin 1982), individual tuatara that have hatched in the past decade could realistically live to experience substantial increases in global mean air temperatures, which could pose a challenge to the tuatara's cold-adapted physiology. Ultimately, if the cognitive abilities of tuatara are plastic in response to environmental temperature, animals exposed to warmer environments under climate change may demonstrate enhanced learning ability. Improved cognitive ability and behavioural flexibility could enable individuals to respond effectively to novel situations and environmental stochasticity, and consequently have an increased chance of survival in unpredictable environments (Amiel \& Shine 2012). Spatial learning ability is likely to be a vital part of the cognitive repertoire of tuatara since they maintain home ranges, defend territories during the mating season, show burrow fidelity, and migrate to rookeries to nest (Gans et al. 1984; Newman 1987; Gillingham \& Miller 1991; Newman et al. 1994; Moore et al. 2009b; Cree 2014). Measuring spatial learning performance and how this may be influenced by ambient temperature is therefore an ecologically relevant assessment of the tuatara's cognitive ability.

\subsection{Thesis aims and organisation}

\subsubsection{Main question}

This is the first time that multiple mainland reintroductions outside of an extant population's latitudinal range have occurred for tuatara, presenting the opportunity to investigate translocation success, plasticity in response to novel climates, and potential 
impacts of climate on aspects of tuatara health (i.e. bacterial communities and parasite loads). Since several sites experience warmer, drier conditions than Stephens Island this allows them to function as proxies for temporal climate change. This study aimed to investigate whether translocated populations of tuatara were progressing successfully in the short term and the possible influences of warmer climates on various aspects of tuatara ecology, with a view to predicting how warming climates expected under anthropogenic climate change might affect tuatara survival and population viability.

\subsubsection{Subquestions}

- Are the tuatara populations translocated to North Island sites in October 2012 progressing favourably in the short term?

- Is the prevalence of Salmonella spp. and Campylobacter spp. in tuatara associated with translocation to warmer climates?

- Are tuatara parasite communities associated with the act of translocation and/or the climates of the translocation sites?

- Do warmer translocation sites affect nocturnal activity or diurnal thermoregulatory behaviour in tuatara?

- Does ambient temperature affect learning ability in tuatara?

\subsubsection{Thesis outline and style}

This thesis includes five research chapters that are formatted for submission to peer reviewed journals. Consequently, there is repetition of information throughout, particularly in the introductory sections, study site descriptions, species descriptions, and some of the methods. However, this style allows the production of several individual, publishable studies while attending to the overall aims and main questions of the thesis. In Chapter Two I evaluated the condition of tuatara populations translocated to sites around New Zealand's North Island in October 2012. This was achieved using several species-appropriate measures of short-term translocation success, which provide important interim measures of how the translocations have progressed. In Chapter Three I investigated the presence of both commensal and potentially pathogenic 
microorganisms, Salmonella spp. and Campylobacter spp., in tuatara to determine whether bacterial prevalence was associated with translocation of individuals to sites with different climates and possible transmission opportunities, and whether these bacteria might pose a risk to tuatara populations under warming climates. Chapter Four investigated the role of translocation and local climate on parasite assemblages with a view to assessing how warmer temperatures under anthropogenic climate change might affect tuatara-parasite interactions and parasite communities. In Chapter Five I assessed the influence of climate on nocturnal activity and diurnal thermoregulatory behaviour at each study site to determine if warmer climates might impose any behavioural/physiological benefits or constraints on translocated populations. Chapter Six details an experimental investigation into the effect of ambient temperature on learning ability in juvenile tuatara, with a view to determining if rising air temperature under anthropogenic climate change might confer some cognitive advantages. In Chapter Seven I deliver an overview of the main findings of this thesis, discuss implications for tuatara conservation, and propose prospective avenues for future research.

This is the first comparative study on the influence of translocation and climate on tuatara behaviour, disease ecology, parasitology and learning ability and utilizes unprecedented large-scale mainland translocations. Findings from this research will inform the management decisions of conservation agencies, be incorporated into sitespecific restoration plans and inform recommendations for future translocation efforts. This research will also inform herpetologists working with other cold-adapted, vulnerable or translocated reptile species and will therefore influence reptile conservation both within New Zealand and internationally.

\subsubsection{Publications}

To date, I have published a case study on the progress of the Cape Sanctuary translocation in the book "Global re-introduction perspectives: 2016. Case-studies from around the globe," published by the IUCN. The full reference is below. Chapters Two and Three area also in preparation to be submitted to the journals PNAS and the New Zealand Journal of Ecology respectively. Chapters Four, Five and Six will also be shortened for publication in scientific journals. 
Full reference for the IUCN case study: Price S, Grayson K, Ward-Smith T \& NJ Nelson. (2016). Re-Introduction of tuatara to a mainland sanctuary within the species historic range in Hawkes Bay, New Zealand. In Soorae, P.S. (ed.). Global Re-introduction Perspectives: 2016. Case-studies from around the globe (pp.xiv+276). Gland, Switzerland: IUCN/SSC Reintroduction Specialist Group and Abu Dhabi, UAE: Environment Agency-Abu Dhabi. 


\section{Chapter Two}

\section{Survival and growth of Cook Strait tuatara (Sphenodon punctatus) following translocation to warmer locations: implications for the temporal effects of climate change}

\subsection{Introduction}

The rate of anthropogenic climate change is a significant conservation concern and the associated environmental impacts are expected to have substantial effects on biodiversity (Parmesan 2006; IPCC 2014). While species might circumvent the risk of extinction by adapting to the changing climate or dispersing to more thermally suitable habitats, species that are range restricted, poor dispersers, or slow to reproduce may be more vulnerable than others and increasing environmental temperatures could pose a significant threat to cold-adapted or ectothermic species (Parmesan 2006; Deutsch et al. 2008; Hare \& Cree 2010; Chen et al. 2011; Hoffman \& Sgro 2011; Miller et al. 2012; Refsnider \& Janzen 2012; Quintero \& Wiens 2013). Obstacles to dispersal can be overcome through interventions like conservation translocations, the intentional, humanmediated movement of a species from one area to another (IUCN/SSC 2013). Such assisted colonisation is a valuable conservation tool that can remove animals from unsuitable habitats, extend a species' range and supplement/restore small or extirpated populations, ultimately reducing the likelihood of species extinction (Griffith et al. 1989; Dodd \& Seigel 1991; Parmesan 2006; Germano \& Bishop 2008; Mawdsley et al. 2009; Thomas 2011; Miller et al. 2012; IUCN/SSC 2013).

A translocation is considered successful when it results in a self-sustaining wild population. Successes have been documented for a wide variety of taxa, demonstrating that this conservation strategy can prove highly beneficial (Sanz 1998; Spalton et al. 1999; Maschinski \& Duquesnel 2007; US Fish And Wildlife Service 2008; Thomas et al. 2009; Soorae 2010, 2011, 2013; Seddon et al. 2012). However, translocations have historically focussed on birds and mammals, with herpetofauna accounting for a much smaller proportion of the published translocation research (Fischer \& Lindenmayer 2000; Seddon et al. 2005). Herpetofauna translocations initially appeared to be particularly challenging after Dodd and Seigel (1991) found that only 19\% of reviewed translocations could be 
deemed successful compared to $63 \%$ and $73 \%$ reported for birds and mammals respectively (Wolf et al. 1996). Poor success rates were attributed to a lack of long-term monitoring, insufficient understanding of the original cause of species decline, and a lack of attention to biological constraints and population genetics. Disease, stress, and reduced reproductive ability have also been identified as contributors to translocation failure (Seigel \& Dodd 2002; Mackintosh \& Briskie 2005; Teixeira et al. 2007; PinterWollman et al. 2009). More recently, Germano and Bishop (2008) found that global success rates for herpetofauna translocations have more than doubled from $19 \%$ to $41 \%$, with the most common reasons for failure now being homing or migratory behaviour and release into inadequate habitat. Similarly, another review found that $60 \%$ of reptile translocation case studies had been categorised as "successful" or "highly successful" (Ewen et al. 2014). While there has undoubtedly been an improvement, it is still apparent that around half of herpetofauna translocations are either inadequately monitored or fail outright (Fischer \& Lindenmayer 2000; Germano \& Bishop 2008; Miller et al. 2014, Romijn \& Hartley 2016). Recent work on the criteria used to determine translocation outcomes found that reported successes in New Zealand were grossly overestimated when using traditional definitions (41.7\%) versus standardised success rates (8.1\%; Miller et al. 2014). This highlights a need for well-defined, standardised success criteria that account for species longevity and duration of post-release monitoring, which, in conjunction with publication of failures/inconclusive results are key to accurate and useful posttranslocation reporting (Dodd \& Seigel 1991; Fischer \& Lindenmayer 2000; Towns et al. 2001; Germano \& Bishop 2008; McCoy et al. 2014; Miller et al. 2014).

As long lifespans and slow sexual maturation are characteristic of many reptile species, it is advised that translocated populations be monitored for at least the time required for an individual to reach sexual maturity before population viability can be accurately evaluated (Germano \& Bishop 2008). Regular monitoring is critical for early detection of potential complications (e.g. predators, disease, dispersal). Four stages have been established to assess translocation success, the first two of which can be used as indicators of short-term progress. The stages are as follows: 1) the survival and growth of founders, 2) evidence of reproduction, 3) population growth through recruitment of locally born offspring, and 4) a self-sustaining population (IUCN/SSC 1998; Nelson et al. 2002b; Germano \& Bishop 2008; Seddon et al. 2012; Miller et al. 2014). 
To maximise the chance of translocation success, it is vital that the initial cause of species decline is addressed and a sufficient number of individuals are released into high quality habitat within the species' historical range that is lacking in potential competitors (Griffith et al. 1989; Dodd \& Seigel 1991; Wolf et al. 1996; Fischer \& Lindenmayer 2000; Germano \& Bishop 2008). The source of the founding population can likewise influence a translocation outcome and there are risks associated with local adaptation, age, and genetic variation (Dodd \& Seigel 1991; Miller et al. 2012). Disease screening is also essential to minimise the potential for pathogen introduction into naïve local populations (Leighton 2002; Gartrell et al. 2006, 2007; Germano \& Bishop 2008; Kock et al. 2010). Wild-caught individuals constitute the majority of reptile translocations, which has been associated with an increased chance of translocation success, though a review by Germano and Bishop (2008) could not confirm this relationship (Griffith et al. 1989; Fischer \& Lindenmayer 2000). An alternative is the use of head-started animals, which are captive-bred or wild-caught and raised in captivity until release (Alberts 2007). Headstarting aims to improve the survival of species with high juvenile mortality by boosting the growth rate and body size of juveniles during their most vulnerable life stage, thereby improving the likelihood that individuals survive to sexual maturity (Haskell et al. 1996; Alberts 2007). While there are disadvantages to using captive-bred individuals, many reptile species are ideal for this strategy owing to cost-effective production, a lack of parental care, and general retention of innate behavioural and physiological traits (Nelson et al. 2002b; Germano \& Bishop 2008). In the case of reptiles, larger individuals tend to have higher survivorship post-release and juvenile tuatara survival has shown to be enhanced by head-starting (Gruber 2007). The increased hatching success of artificially incubated vs. wild incubated eggs means artificial incubation can also reduce pressure on the source population as a greater number of individuals can be obtained for translocation with minimal removal of eggs (Thompson 1990; Cree et al. 1995; Thompson et al. 1996; Nelson et al. 2004b). Translocations consisting of head-started juvenile and adult animals can be advantageous due to a reduced vulnerability to predation and unfavourable environmental conditions, which can reduce mortality in founding populations (Haskell et al. 1996; Alberts 2007; Germano \& Bishop 2008).

The tuatara is a long-lived (known to live over 100 years), cold-adapted, New Zealand endemic reptile and the sole extant representative of the order Rhynchocephalia 
(Saint Girons 1980; Barwick 1982; Dawbin 1982; Evans 2003). Holocene subfossil records indicate that tuatara were once widespread throughout New Zealand (Chapter One, Figure 1.1), but with the arrival of human settlers $~ 750$ years ago came habitat change and introduced mammalian predators that resulted in extirpation from the mainland and the restriction of tuatara to predator-free offshore islands (Cree \& Butler 1993; Anderson 1996; Holdaway \& Worthy 1997). The long-term goal of the Tuatara Recovery Group was to maintain the genetic diversity of tuatara by reinstating wild populations throughout their historical range (Gaze 2001). As most populations survive on islands where dispersal is not feasible, and as the initial cause of decline from mainland areas (i.e. mammalian predators) has not been eliminated, human-mediated translocations to predator-free locations have been essential to tuatara conservation.

The first contemporary tuatara conservation translocation occurred in 1995 when adult and juvenile animals were moved within the Cook Strait from North Brother Island to the rodent-free Titi Island (Nelson et al. 2002b). Since then, significant progress has been made through the eradication of predatory mammals on multiple additional islands suitable for tuatara, which has facilitated further translocations (Towns et al. 2001; Nelson et al. 2002b; Gartrell et al. 2006; Miller et al. 2010). Because tuatara are long-lived and females reproduce every 4-5 years on average, even early translocations have not yet reached the stage at which population viability, and ultimately translocation success, can be ascertained (Castanet et al. 1988; Cree 1994). While recent reports on short-term success are not available for all tuatara translocations, in several cases post-release monitoring has shown populations to be progressing favourably with high recaptures, growth and evidence of reproduction observed at many sites (Nelson 1998; Owen 1998; MacBrayne 2002; Nelson et al. 2002b, 2008; Gaze \& Cash 2008; Miller et al. 2010; Cree 2014).

Despite favourable results so far, tuatara are still at risk from rising air temperatures, while their ancestors were able to survive through past climate change events (e.g. the "Oligocene drowning") there is a lack of information on how physiologically similar extant populations are to their extirpated ancestors; therefore we cannot assume that tuatara have retained attributes that enabled their ancestors to survive past climate change (Nelson et al. 2002b; Gibbs 2006; Mitchell et al. 2008; Jones et al. 2009; Miller et al. 2012). Such uncertainty and the severe limits on the current ability 
of tuatara to disperse or move to different habitats, makes understanding their responses to a warming climate necessary for direct conservation management efforts and to ensure the future of the species.

In October 2012, several conservation translocations moved 176 adult tuatara from Takapourewa/Stephens Island in the Cook Strait to four sites around New Zealand's North Island, all of which lie within their historical range: Maungatautari Ecological Reserve, Whangaokeno/East Island, Cape Sanctuary and Young Nicks Head Sanctuary (Chapter One, Figure 1.1; Worthy \& Holdaway 2002; Wood 2009). Adult and juvenile tuatara were also moved from Takapourewa/Stephens Island to Orokonui Ecosanctuary in New Zealand's South Island. A research group at Otago University in Dunedin is monitoring this population and several studies have been published on this population's progress since release (Jarvie et al. 2014, 2015, 2016). Each translocation site is latitudinally distant from the source site and the diverse environmental conditions at each site offer the opportunity to study tuatara across a range of new environments. The comparatively warmer, drier climates (with the exception of Maungatautari), which are on average $2-4^{\circ} \mathrm{C}$ warmer than the source site during summer months, provide surrogates for temporal climate change, enabling an assessment of the ability of tuatara to adjust to a warming climate (Figures 2.1 and 2.2). A population of tuatara translocated to ZEALANDIA $^{\mathrm{TM}}$ (formerly Karori Sanctuary) in Karori, Wellington in 2005 and 2007 also enables a comparison of the impact of translocation alone, rather than a change in climate. The broad active temperature range of tuatara means they can be considered a reasonably thermally robust species, although death due to heat stress has been recorded at air temperatures over $25^{\circ} \mathrm{C}$ in captivity, and there is a risk that local adaptation to the Stephens Island climate might influence their ability to establish successfully (Blanchard 2002; Miller et al. 2012; B. Gartrell, pers. comm.). Equally, local species may present other risks (e.g. pathogen transmission, competition, conflict), so short-term monitoring will be vital to evaluating the progress of these translocations as well as quickly identifying potential threats to the translocated tuatara (Kutz et al. 2005; Atkins \& Travis 2010; Jankowski et al. 2010; Miller et al. 2012; Refsnider 2013).

This study aimed to evaluate the short-term success and progress of translocated tuatara populations and assess the possible influence of warmer sites on these populations. This was achieved by monitoring: 1) survival of founders, as indicated by 
cumulative recapture numbers, 2) growth of founders, measured as increases in both mass and snout-vent length and, 3) reproduction, as evidenced by observations of nests, eggs and hatchling tuatara. We used success indicators previously employed by the Tuatara Recovery Group and following previous tuatara translocations to assess shortterm progress (Newman 1998; Nelson et al. 2008; Miller et al. 2010). For a translocation to be judged as progressing favourably these criteria require the recapture of at least $30 \%$ of founders within three years of release, growth of founders, and evidence of reproduction within 10 years of release (Nelson et al. 2002b, 2008). While these early measures of the translocations cannot be used to judge the overall success or failure of the new populations, these data will provide an important interim measure of the translocations' progress. 

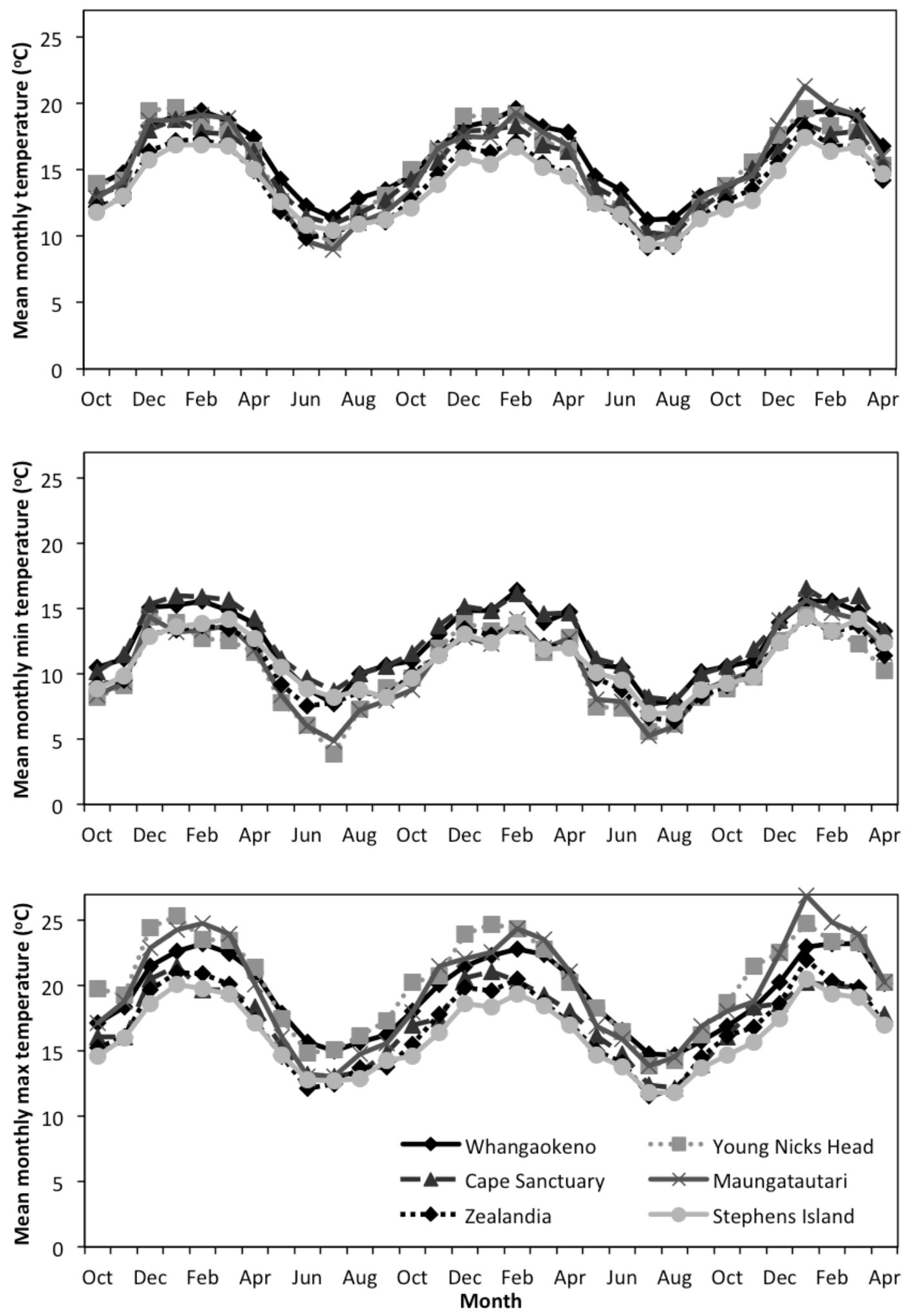

Figure 2.1 Mean, mean maximum and mean minimum monthly air temperatures per site from translocation to final survey: October 2012 - April 2015. Temperature data were obtained from the NIWA (National Institute of Water and Atmospheric Research Ltd.) CliFlo database (http://www.cliflo.niwa.co.nz) and references weather stations in closest proximity to the translocation sites for which data were available (see Appendix 1, Table A1.1). Data are not site specific but representative of regional temperatures. 

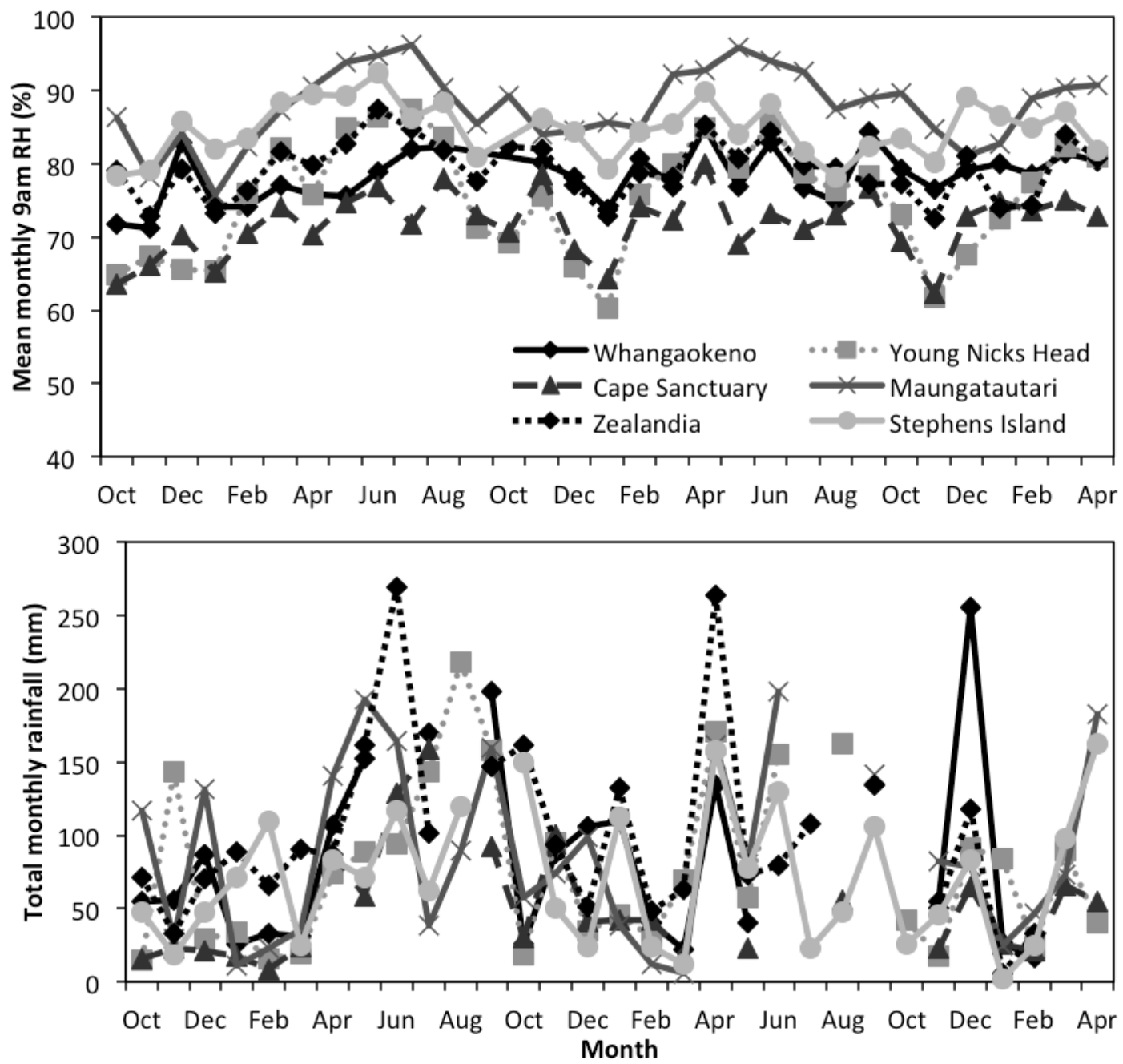

Figure 2.2 Mean monthly 9am relative humidity $(\mathrm{RH})$ and total rainfall per site from translocation to final survey: October 2012 - April 2015. RH and rainfall data were obtained from the NIWA (National Institute of Water and Atmospheric Research Ltd.) CliFlo database (http://www.cliflo.niwa.co.nz) and references weather stations in closest proximity to the translocation sites for which data were available (see Appendix 1, Table A1.1) Data are not site specific but representative of regional humidity. Data were missing for some months at some stations so lines do not connect all data points.

\subsection{Methods}

\subsubsection{Study sites}

\subsubsection{Source site}

Stephens Island/Takapourewa is a pest-free, 150 ha offshore island Nature Reserve in the Marlborough Sounds $\left(40^{\circ} 40^{\prime} 15.4^{\prime \prime S} 173^{\circ} 59^{\prime} 51.7^{\prime \prime} \mathrm{E}\right)$ and is a site of high conservation significance (East et al. 1995; DOC 2006). Stephens Island was historically densely forested, but after acquisition by the New Zealand Government and the installation of a 
lighthouse and keepers, by 1894 an estimated $90 \%$ of the forest cover had been destroyed and reduced to patches of remnant forest, scrubland, and pasture (East et al. 1995; see Appendix 2, Figure A2.1 for images of habitat). Following automation of the lighthouse, the keepers were removed from the island in 1989 and Stephens Island was returned to the ownership of the local Māori, Ngāti Kōata, who gifted it back to the Crown in 1994 (East et al. 1995; Brown 2000). There has since been a collaborative effort between Ngāti Kōata and the Department of Conservation (DOC) to ecologically restore the island.

Stephens Island is home to an estimated 30,000 - 50,000 tuatara, the largest population in the world, alongside multiple species of native geckos, skinks and birds (Newman 1982; Gans 1983; East et al. 1995; Gaze 2001). Among the numerous seabirds there are approximately one million nesting fairy prions (Pachyptila turtur) inhabiting the island, the droppings of which have contributed to a rich soil that supports an abundance of invertebrate fauna that has likely helped the tuatara population to flourish (East et al. 1995; Gaze 2001). Such a high density of tuatara (approximately $250-2,700$ per hectare depending on habitat) and high genetic diversity makes the Stephens Island population an ideal source of founders for translocations (Moore 2008, Moore et al. 2009b).

\subsubsection{Translocation sites}

Cape Sanctuary is a privately owned, 2,500 ha sanctuary in the Hawkes Bay region $\left(39^{\circ} 39^{\prime} 40.0^{\prime \prime} \mathrm{S} 177^{\circ} 03^{\prime} 32.0^{\prime \prime} \mathrm{E}\right)$. Within this site lies the "seabird cell", a pest-free, 1.5 ha enclosure surrounded by a $610 \mathrm{~m}$ pest-proof fence (McLennan 2013). Within the seabird cell is a separate approximately $2,000 \mathrm{~m}^{2}$ enclosure surrounded by a $1 \mathrm{~m}$ tall wood and shade cloth fence (T. Ward-Smith, pers. comm.). Forty adult tuatara (20 males, 20 females) were released into artificial burrows (bore holes, wooden boxes and buried terracotta pipes) within this enclosure. One female was suspected to be gravid. Artificial hides had also been constructed from terracotta tiles, plastic pipes and log piles to provide alternative means of shelter. Extensive replanting of native flora at the site has restored native shrubs and trees, including mature flax (Phormium tenax), Coprosma sp. and manuka (Leptospermum scoparium), as well as more open areas of non-native grassland, which provided a variety of habitats for tuatara (see Appendix 2, Figure A2.1). Twenty 
head-started juvenile tuatara were also released into a separate, identically-constructed $2,000 \mathrm{~m}^{2}$ enclosure within the seabird cell.

Young Nicks Head Sanctuary is located on the privately owned Young Nicks Head Peninsula in Poverty Bay $\left(38^{\circ} 45^{\prime} 27.4^{\prime \prime S} 177^{\circ} 57^{\prime} 24.3^{\prime \prime E}\right)$. The 35 ha, pest-free site is surrounded on three sides by steep cliffs and protected by a $500 \mathrm{~m}$ long, $2 \mathrm{~m}$ high $\mathrm{XCluder}^{\circledR}$ pest-proof fence, which runs from cliff to cliff across the peninsula (Sawyer \& Fogle 2010). Forty-two adult tuatara (23 males, 19 females) were released into preprepared, numbered artificial burrows in the vicinity of the "valley track" within the enclosure. Two females were suspected to be gravid. Artificial burrows were made of corrugated plastic pipes, partially buried to leave one end exposed and allow access. Extensive regeneration planting of $\sim 46,000$ native saplings prior to the translocation has restored a closed-canopy, native, coastal forest, providing ample cover for tuatara (see Appendix 2, Figure A2.1). Twenty head-started juveniles were also released into a separate $50 \mathrm{~m}^{2}$ enclosure within the 35 ha site that had been installed with 20 artificial burrows (wooden boxes with access tunnels) and planted with native vegetation. The enclosure was constructed from wood and wire mesh to allow the passage of invertebrates into the enclosure and protect the juveniles from natural predators.

Whangaokeno/East Island is a 13 ha protected offshore island Wildlife Refuge Reserve $\sim 2 \mathrm{~km}$ off the coast of New Zealand's East Cape (37 $\left.41^{\prime} 27.7^{\prime \prime} \mathrm{S} 178^{\circ} 34^{\prime} 31.9^{\prime \prime} \mathrm{E}\right)$, beyond the swimming distance of introduced mammalian pests (King 1990; Nelson et al. 2002b). Forty-four adult tuatara ( 25 males, 19 females - none thought to be gravid) were released into pre-prepared artificial burrows (bore holes) at three locations around the island. Several species of seabird have colonies on the island so an extensive network of seabird burrows, as well as patches of regenerated native vegetation and open areas of grassland, offered tuatara a range of habitats to disperse into (Thorsen 2004; see Appendix 2, Figure A2.1). Co-habitation with seabirds is also thought to directly benefit tuatara through the provision of burrows and food (i.e. seabird eggs and chicks) and indirectly through an increase in invertebrate abundance (Walls 1978, 1980; Markwell 1998; Blair et al. 2000; Ussher 2003; Baling et al. 2013b).

Maungatautari is a 3,400 ha, pest-free, mainland island sanctuary in the Waikato region (38 01'43.5"S $\left.175^{\circ} 34^{\prime} 29.4^{\prime \prime} \mathrm{E}\right)$. A $47 \mathrm{~km} \mathrm{XClude{ } ^ { \circledR }}$ pest-proof fence encircles the mountain. On the southern side of this sanctuary is a separately fenced, pest-free site 
known as the Tautari Wetland, which contains a $900 \mathrm{~m}^{2}$ tuatara enclosure. Fifty adult tuatara (30 males, 20 females) were released at Maungatautari, with 30 (18 males, 12 females) released into pre-prepared artificial burrows in a 35 ha enclosure on the main mountain and 20 (12 males, 8 females) released into pre-prepared artificial burrows in the smaller Tautari Wetland enclosure. Two females were suspected to be gravid. Artificial burrows were constructed from buried plastic containers with two corrugated plastic pipes leading to the surface to allow access. Mature forest on the main mountain and a variety of native shrubs, mature trees and open ground in the wetland enclosure provide a range of habitats for tuatara (see Appendix 2, Figure A2.1).

The tuatara population at ZEALANDIA ${ }^{\mathrm{TM}}$ (formerly Karori Sanctuary) in Karori, Wellington $\left(41^{\circ} 17^{\prime} 26.9^{\prime \prime S} 174^{\circ} 45^{\prime} 11.2^{\prime \prime} \mathrm{E}\right)$ was monitored for comparison purposes throughout this study. The sanctuary has an area of 225 ha and is surrounded by an 8.6 km, 2.2 m high pest-proof fence. Between 2005 and 2007, 200 adult tuatara were translocated to this site from Stephens Island and released into artificial, corrugated pipe burrows within a fenced, 1 ha enclosure known as the Research Area, as well as at two sites in the wider sanctuary, the Valley View/Union Mine Tracks and Turbine Track. The release sites received 60, 40 and 100 tuatara respectively. There has been extensive regeneration of native flora within ZEALANDIA, which has restored a closed canopy forest in much of the valley (see Appendix 2, Figure A2.1), and over 40 locally or nationally threatened species of native flora and fauna have been released into the valley. The preliminary translocation in December 2005 of 70 adult tuatara was monitored for one year (January-December 2006) following release by McKenzie (2007). These data have also been used for comparative purposes.

\subsubsection{Founding population and translocations}

The large tuatara population on Stephens Island means that the removal of 176 animals for translocation is unlikely to negatively impact the population and may instead relieve interspecific and intraspecific competition at locations where animals were captured (Newman 1982; Moore et al. 2007). Animals were captured at night, when tuatara are most active, and placed into individual cloth bags. Sex, parasite load (ticks, Amblyomma sphenodonti, and mites, Neotrombicula spp.), and measurements of snout-vent length 
(SVL), vent-tail length (VTL), tail regeneration (if present) and mass were recorded. Each animal was thoroughly checked for signs of injury or infection and any identifying features (e.g. toe clips). Captures were performed in October when some females were likely to be carrying shelled eggs/nearing oviposition and all adult females were palpated to determine gravidity. If a translocated female is carrying the offspring of a nontranslocated male this can benefit translocated populations by improving genetic diversity and the effective founding population size at the new site (Nelson et al. 2002b). Blood samples, cloacal swabs and faecal samples were collected for disease and intestinal parasite screening to obtain pre-release health data and assess the risk of disease transmission, although analyses could not be performed prior to translocation. To enable the identification of translocated individuals, each animal was fitted with a passive integrated transponder (PIT) microchip prior to transport, which was inserted subcutaneously on the left side of the body, just forward of the inguinal fold. The resulting hole was then sealed with Vetbond ${ }^{\mathrm{TM}}$ tissue adhesive. Past identification techniques have included toe clipping and bead-tagging so some individuals also had these identifying marks. On the day of departure from Stephens Island each animal was placed into its own $500 \mathrm{~mm}$ long aerated postal tube, labelled with its individual PIT microchip number, along with a piece of damp paper towel to maintain humidity. All animals were transported from Stephens Island to Wellington airport by helicopter and from there to the respective release sites by helicopter, aeroplane, and/or car. Ambient temperature during transport was maintained at $15-18^{\circ} \mathrm{C}$. Tuatara were released at translocation sites within 12 hours of departure from Stephens Island.

Head-started juvenile tuatara were translocated to Cape Sanctuary and Young Nicks Head Sanctuary in March 2012. These animals had been hatched from eggs collected from Stephens Island and incubated at Victoria University of Wellington in Wellington, New Zealand (DOC permit NM-18922-CAP). According to standard protocols followed in previous studies, juveniles were then raised at a captive-rearing facility in semi-natural, outdoor enclosures until $\sim 5$ years of age (Nelson et al. 2002b; Gartrell et al. 2006). The juveniles were transported from the captive-rearing facility in Wellington to Cape Sanctuary and Young Nicks Head Sanctuary by car and released into artificial burrows in their respective enclosures within 24 hours. 


\subsubsection{Opportunistic monitoring}

Four post-release monitoring trips were conducted in spring and summer/autumn between November 2013 and May 2015 to survey adult founders. Due to access limitations, Whangaokeno and Stephens Island were only visited twice, in the austral summer/autumn of 2014 and 2015, and Young Nicks Head was visited once in the austral summer of 2015 (Table 2.1). Visits ranged from 1 to 10 days in duration, with longer visits designed to allow for the collection of behavioural data from females as well as postrelease monitoring information. During each trip, nights would be spent searching the site for animals (Table 2.1). Searches typically ran from $8 \mathrm{pm}$ until between midnight and 2am depending on catch success and weather. The number of nights dedicated to post-release monitoring depended on the size and layout of the site and whether alternative survey work was also taking place that might be disturbed. Searches were conducted using head torches with white light. Both artificial and natural burrows were visually monitored and investigated for the presence of tuatara and surrounding areas of vegetation methodically searched. Captured tuatara were placed temporarily into cloth bags and moved a short distance for the purpose of data collection or had measurements and samples taken immediately at the capture location. If a tuatara was moved from its capture location, a numbered reflective stake was placed in the ground where the animal had been found; this allowed for the release of animals at their exact capture location after data collection. The following measurements were taken from captured tuatara: SVL, VTL, tail regeneration length (if present) and mass. GPS location, sex, PIT identification number, toe-clip/bead tag identification (if present) were also recorded. Animals were visually checked for any signs of injury or infection. After sampling, all animals were marked dorsally, at the base of the skull, with a black xylene-free marker pen. This enabled identification of already-captured animals and prevented unnecessary disturbance during the rest of the survey. Sites were explored for evidence of nesting/breeding where possible.

Head-started juvenile founders were surveyed twice at Young Nicks Head Sanctuary, in spring 2013 and summer 2014, and twice at Cape Sanctuary, in summer 2013 and 2015. Surveys were conducted during daylight as juvenile tuatara are most active above-ground during the day (Terezow et al. 2008). Surveys at Young Nicks Head 
Sanctuary were 2.5 hours (spring 2013) and five hours (summer 2014) long, during which two to four people methodically searched burrows and vegetation for animals. Master's students from the Victoria University of Wellington New Zealand Conservation Practice course conducted the Cape Sanctuary juvenile tuatara surveys in 2013 and 2015. The Cape Sanctuary juvenile enclosure was searched for approximately four (summer 2013) and five (summer 2015) hours per trip (Benavidez et al. 2013; Gibson et al. 2015; V. Stenhouse pers. comm.). The following were recorded for all captured juveniles: SVL, VTL, tail regeneration length (if present), mass, GPS location, sex and toe-clip identification (Benavidez et al. 2013; Gibson et al. 2015). All juveniles were visually checked for signs of injury or infection.

\subsubsection{Data and statistical analyses}

All statistical analyses were performed in R v. 3.2.2 (R Core Team 2015). I used the package "Ime4" to execute linear mixed-effects models (LMMs) and generalised linear models (GLMs; Bates et al. 2016). I used GLMs with binomial distributions to determine if the size of an individual (measured by mass (g)) on release could have influenced whether or not an individual was recaptured during any of the four post-release surveys. This test was based on the hypothesis that an individual of smaller body mass could have suffered a competitive disadvantage following release and not been recaptured because it had died.

I used LMMs to estimate the differences in both adult and juvenile SVL growth rates between study sites following release using an interaction term between the study site (a four-level categorical variable for adults or two-level for juveniles i.e. the release sites) and months since release. Tuatara individual ID was included as a random factor. Kenward-Rogers approximations were run using the package "pbkrtest" to obtain parameter-specific p-values (Halekoh \& Højsgaard 2012). Only tuatara that had been recaptured and measured following release were included in the models of SVL growth. As growth rates are expected to slow as juveniles reach maturity (Dawbin 1982), translocated "adults" with an SVL of less than $170 \mathrm{~mm}$ on release were not included in the SVL growth analysis as animals of $170-180 \mathrm{~mm} \mathrm{SVL}$ are considered non-sexually mature sub adults (Blanchard 2002). Similarly, any juveniles that were found to have attained SVLs of $180 \mathrm{~mm}$ or above during a survey had their data for that survey removed, 
Table 2.1 Sites surveyed, area, adult tuatara population size/density (unless stated as juveniles), search effort and the number of individuals captured per survey. Tuatara density on Stephens Island is estimated at 200 per ha for a total estimated population of 30,000 and 333 per ha for a total estimated population of 50,000 (see Chapter 3, section 3.2.5 for details). Seasonal surveys were classified as follows: spring: November-December; summer: February; autumn: March-May. Surveys were not conducted over June-August (winter) or DecemberJanuary (early-mid summer).

\begin{tabular}{|c|c|c|c|c|c|c|c|c|c|c|}
\hline & Site & $\begin{array}{l}\text { Area } \\
\text { (ha) }\end{array}$ & $\begin{array}{l}\text { Pop. } \\
\text { size }\end{array}$ & $\begin{array}{l}\text { Pop. density } \\
\text { (no. per ha) }\end{array}$ & $\begin{array}{c}\text { Season } \\
\text { visited }\end{array}$ & $\begin{array}{c}\text { No. } \\
\text { days/nights }\end{array}$ & $\begin{array}{c}\text { No. } \\
\text { people }\end{array}$ & $\begin{array}{l}\text { No. "person } \\
\text { nights" }\end{array}$ & No. captures & $\begin{array}{l}\text { No. captures per } \\
\text { "person night" }\end{array}$ \\
\hline \multirow{4}{*}{\multicolumn{2}{|c|}{ Cape Sanctuary }} & \multirow{4}{*}{0.2} & \multirow{4}{*}{40} & \multirow{4}{*}{200} & Spring '13 & 3 & 4 & 12 & 19 & 1.6 \\
\hline & & & & & Autumn '14 & 6 & $2-4$ & 14 & 21 & 1.5 \\
\hline & & & & & Spring '14 & 3 & 4 & 12 & 23 & 1.9 \\
\hline & & & & & Autumn '15 & 3 & $4-5$ & 13 & 23 & 1.8 \\
\hline \multirow{4}{*}{\multicolumn{2}{|c|}{$\begin{array}{l}\text { Maungatautari } \\
\text { Tautari wetland }\end{array}$}} & \multirow{4}{*}{0.09} & \multirow{4}{*}{20} & \multirow{4}{*}{222} & Spring '13 & 1 & 3 & 3 & 13 & 4.3 \\
\hline & & & & & Autumn '14 & 4 & 2 & 8 & 14 & 1.8 \\
\hline & & & & & Spring '14 & 3 & $2-6$ & 12 & 14 & 1.2 \\
\hline & & & & & Autumn '15 & 3 & $2-5$ & 12 & 15 & 1.3 \\
\hline \multirow{3}{*}{\multicolumn{2}{|c|}{$\begin{array}{l}\text { Maungatautari } \\
\text { mountain enclosure }\end{array}$}} & \multirow{3}{*}{35} & \multirow{3}{*}{30} & \multirow{3}{*}{0.86} & Spring '13 & 1 & 3 & 3 & 4 & 1.3 \\
\hline & & & & & Spring '14 & 1 & 4 & 4 & 4 & 1.0 \\
\hline & & & & & Autumn '15 & 1 & 4 & 4 & 8 & 2.0 \\
\hline \multirow{2}{*}{\multicolumn{2}{|c|}{ Whangaokeno }} & \multirow{2}{*}{13} & \multirow{2}{*}{44} & \multirow{2}{*}{3.39} & Summer '14 & 4 & 4 & 16 & 14 & 0.9 \\
\hline & & & & & Summer '15 & 2 & 5 & 10 & 8 & 0.8 \\
\hline \multirow{2}{*}{$\begin{array}{l}\text { Young } \\
\text { Nicks }\end{array}$} & \multirow{2}{*}{ Juveniles } & \multirow{2}{*}{0.005} & \multirow{2}{*}{20} & \multirow{2}{*}{4,000} & Spring '13 & 1 & 4 & 4 & 13 & 3.3 \\
\hline & & & & & Summer '14 & 1 & 4 & 4 & 20 & 5.0 \\
\hline Head & Adults & 35 & 42 & 1.2 & Summer '15 & 1 & 3 & 3 & 7 & 2.3 \\
\hline \multirow{4}{*}{\multicolumn{2}{|c|}{ ZEALANDIA }} & \multirow{4}{*}{225} & \multirow{4}{*}{200} & \multirow{4}{*}{0.89} & Spring '13 & 5 & $2-3$ & 14 & 23 & 1.6 \\
\hline & & & & & Autumn '14 & 6 & $4-6$ & 30 & 24 & 0.8 \\
\hline & & & & & Spring '14 & 5 & $3-6$ & 21 & 19 & 0.9 \\
\hline & & & & & Autumn '15 & 5 & $5-7$ & 18 & 24 & 1.3 \\
\hline \multirow{2}{*}{\multicolumn{2}{|c|}{ Stephens Island }} & \multirow{2}{*}{150} & \multirow{2}{*}{$\begin{array}{c}30- \\
50,000\end{array}$} & \multirow{2}{*}{$200-333$} & Autumn '14 & 6 & 3 & 18 & 35 & 1.9 \\
\hline & & & & & Autumn '15 & 7 & 3 & 21 & 43 & 2.0 \\
\hline
\end{tabular}


as they were no longer within the juvenile size range and would be expected to exhibit slower growth rates. Any juveniles of indeterminate sex were not included in the analyses. Individual body condition over time post-release was also assessed in adult tuatara. Body condition indices $(\mathrm{BCl})$ were calculated separately for each survey season as the residuals of a linear regression of log-transformed mass and log-transformed SVL, a method that has been used in previous studies on tuatara (Hoare et al. 2006; Moore et al. 2007; Godfrey et al. 2010a). The $\mathrm{BCl}$ scores used in this study met the assumption of linearity $\left(R^{2}>0.82\right)$ for all survey seasons.

Due to the small sample sizes obtained from Whangaokeno and Young Nicks Head in the final post-release survey I was unable to investigate the potential influence of sitespecific factors like population density and air temperature on tuatara growth postrelease. 


\subsection{Results}

\subsubsection{Survival of adult founders}

Cumulative founder recapture rates for the October 2012 translocation sites over 2.5 years post-release ranged from $17 \%$ to $95 \%$ of the adult founding populations and $75 \%$ to $100 \%$ of the juvenile founding populations (Table 2.2, Figure 2.3). The deaths of two adult males at Cape Sanctuary during this study, one from unknown causes and one during surgery following a traumatic eye injury, reduced the number of male recaptures to 16 of a possible 18 individuals (89\%) and the total number of recaptured founders to 35 of a possible 38 individuals (92\%, Table 2.2 ). Multiple recapture numbers were calculated as the number of recaptured animals that were caught more than once during the study and were variable across the sites (Table 2.2). Multiple recaptures may have been higher on Stephens Island but many individuals lacked identifying features (e.g. PIT tags, beads, toeclips, etc).

I found that size at release in October 2012 did not affect the chance that an individual was recaptured in a subsequent survey in the Cape Sanctuary, Young Nicks Head or Maungatautari adult populations ( $p>0.05$ ), but did have a significant influence on whether or not an individual was recaptured in the Whangaokeno population $(x 2(1)=$ 6.445 , model $p=0.011$, estimate $=0.008 \pm 0.003, z=2.390, p=0.017)$. The mean mass on release of individuals that were not recaptured was $281 \mathrm{~g}( \pm 81 \mathrm{~g})$, whereas the mean mass on release of individuals that were not recaptured was $359 \mathrm{~g}( \pm 113 \mathrm{~g}$ ), a difference of $77 \mathrm{~g}$. 
Table 2.2 Number/percentage of adult population captured and breakdown of the percentage of males and females captured. Data are for adult animals unless stated otherwise. Multiple recaptures refers to the percentage of the recaptured population caught on more than one occasion during the study. An * indicates altered capture and population values as a result of two known deaths of adult male tuatara at Cape Sanctuary. Percentage of population captured on Stephens Island is estimated at $0.25 \%$ for a total estimated population of 30,000 and $0.15 \%$ for a total estimated population of 50,000 (Newman 1982; Gans 1983). Percentage of male and female population captured not shown as total numbers of males and females are not known. Cape Sanctuary juvenile data were obtained from Benavidez et al. (2013) and Gibson et al. (2015) as described in Methods 2.2.3. Sex of one Young Nicks Head juvenile was unknown so this individual was not included in percentage recapture calculations of males and females. ZEALANDIA data for 2006 was obtained from McKenzie (2007).

\begin{tabular}{|c|c|c|c|c|c|c|c|}
\hline Site & $\begin{array}{l}\text { Population } \\
\text { size }\end{array}$ & No. captured & $\begin{array}{c}\text { Search effort } \\
\text { (no. "person } \\
\text { nights") }\end{array}$ & $\begin{array}{c}\text { Percentage of } \\
\text { population } \\
(\%)\end{array}$ & $\begin{array}{c}\text { Percentage } \\
\text { of females } \\
(\%)\end{array}$ & $\begin{array}{l}\text { Percentage } \\
\text { of males (\%) }\end{array}$ & $\begin{array}{c}\text { Multiple } \\
\text { recaptures } \\
(\%)\end{array}$ \\
\hline Cape Sanctuary & $60(* 58)$ & $52(* 50)$ & 51 & $87(* 86)$ & 88 & $86(* 85)$ & $73(* 76)$ \\
\hline Adults & $40(* 38)$ & $37(* 35)$ & 51 & $93(* 92)$ & 95 & $90(* 89)$ & $76(* 80)$ \\
\hline Juveniles & 20 & 15 & na & 75 & 75 & 75 & 67 \\
\hline Maungatautari & 50 & 32 & 46 & 64 & 55 & 70 & 59 \\
\hline Tautari wetland & 20 & 19 & 35 & 95 & 100 & 92 & 89 \\
\hline $\begin{array}{l}\text { enclosure } \\
\text { Mountain enclosure }\end{array}$ & 30 & 13 & 11 & 43 & 25 & 56 & 15 \\
\hline Whangaokeno & 44 & 17 & 26 & 39 & 26 & 48 & 29 \\
\hline Young Nicks Head & 62 & 27 & 11 & 44 & 45 & 40 & - \\
\hline Adults & 42 & 7 & 3 & 17 & 11 & 22 & - \\
\hline Juveniles & 20 & 20 & 8 & 100 & 100 & 100 & 65 \\
\hline \multicolumn{8}{|l|}{ ZEALANDIA } \\
\hline 2006 & 70 & 44 (+12 seen) & $>30$ & 80 & 86 & 74 & - \\
\hline 2013-2015 & 200 & 48 & 83 & 24 & 24 & 24 & 48 \\
\hline Stephens Island & $30-50,000$ & 76 & 39 & $0.15-0.25$ & na & na & 3 (min.) \\
\hline
\end{tabular}



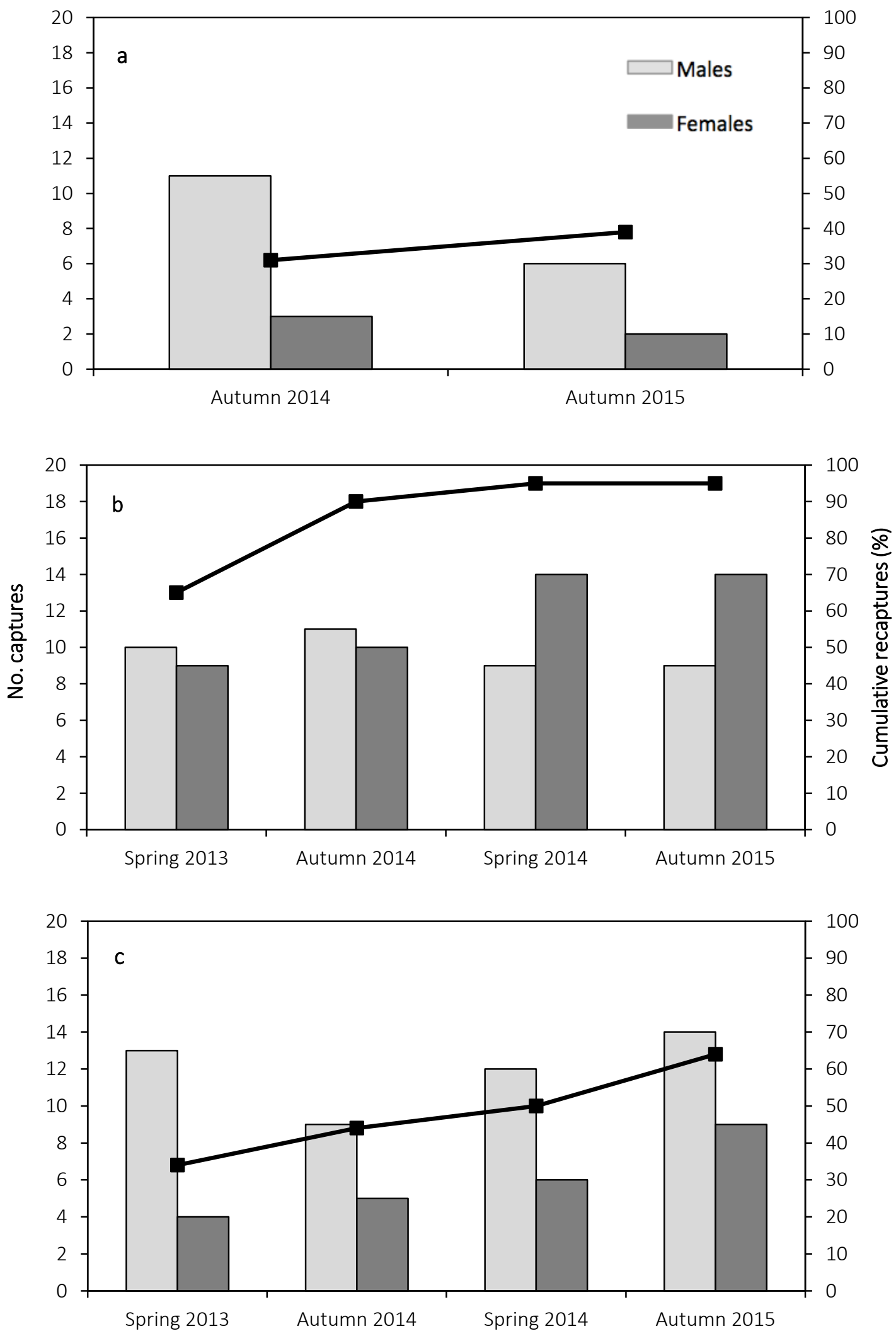

\section{Monitoring season}

Figure 2.3 Adult capture numbers per survey from spring 2013 to autumn 2015 for October 2012 translocation sites visited more than once. Bars show number of individuals captured per survey for males and females and black lines show the cumulative number of recaptures as a percentage of the founding population since release. Graph a = Whangaokeno; $b=$ Cape Sanctuary; $c=$ Maungatautari. 


\subsubsection{Growth and body condition of founders}

Analyses of SVL growth and changes in body condition only included animals that had been recaptured at least once during post-release surveys. Growth rates were not calculated for Stephens Island tuatara as few had identification (i.e. PIT tags, bead tags) so repeat captures could not be identified across surveys. I found that, while there were no significant differences between the mean SVL size of recaptured males on release at the different translocation sites ( $p>0.05$ ), the mean SVL size of females released at Whangaokeno was significantly smaller than SVL on release for the recaptured ZEALANDIA females (estimate $=26.88 \pm 9.64, t=2.79, p=0.007$ ), but not significantly different to females at any of the other translocated sites $(p>0.05)$.

In the 2.5 years since release skeletal growth, as measured by SVL growth, has been negligible at three of the four translocation sites (Figure 2.4). Estimated mean SVL growth rates total less than $1 \mathrm{~mm}$ a year in most populations and, in some cases, indicate a mean decrease in SVL, with SVL growth rates per site ranging from -0.25 to $6.76 \mathrm{~mm}$ year $^{-1}$ for males and -1.31 to $4.37 \mathrm{~mm}$ year ${ }^{-1}$ for females (see Appendix 2, Table A2.1). As a negative SVL growth rate is biologically infeasible, these negative mean growth estimates likely represent SVL measurements taken across surveys that fall within the margin of error expected when taking detailed measurements by hand from a live animal. For instance, a Cape Sanctuary adult male was recorded as having a SVL of $247 \mathrm{~mm}$ in one survey, and $245 \mathrm{~mm}$ in the following survey, which would indicate negative growth, but is more likely due to a measurement error. In the case of all sites showing negative or minimal growth rates, it is likely that tuatara released at these sites have, on average, experienced very low or no SVL growth following release. Individual SVL change between release in 2012 and the final SVL measurement of those captured in the autumn 2015 survey range from -9 to $+9 \mathrm{~mm}$ at Cape Sanctuary, -10 to $+33 \mathrm{~mm}$ at Maungatautari, +6 to $+18 \mathrm{~mm}$ at Whangaokeno, and -9 to $+9 \mathrm{~mm}$ at Young Nicks Head. The highest SVL growth rates were observed in the Whangaokeno population (male: $6.76 \mathrm{~mm}$ year $^{-1}$; female: $4.37 \mathrm{~mm}_{\text {year-1 }}{ }^{-1}$ ) and were significantly higher than growth rates observed in any other translocated population, including ZEALANDIA ( $p<0.05$; Figure 2.5; see Appendix 2, Table A2.2 for statistical details). Growth rates at Cape Sanctuary were also significantly lower than those observed at ZEALANDIA (estimate $=0.16 \pm 0.05, t=3.04, p=0.003$ ), but 
growth rates did not differ significantly among any of the other translocation sites $(p>$ 0.05). However, it is important to note that sample sizes for Young Nicks Head and Whangaokeno females in post-release surveys were low ( $n=2-3$ per site per survey), so the calculated mean growth rates may not be representative of the entire population.

The data on body conditions at each site indicate general increases in individual body condition following release (Figure 2.6). I found that $61 \%$ of recaptured Maungatautari individuals, $71 \%$ of recaptured Young Nicks Head individuals, and $88 \%$ of recaptured Whangaokeno individuals showed increases in $\mathrm{BCl}$ scores following release (based on measurement data from each individual's most recent recapture). In contrast, $\mathrm{BCl}$ scores for Cape Sanctuary show that $72 \%$ of the adult population experienced a decline in body condition post-release (Figure 2.6). By comparison, $62 \%$ of the animals released at ZEALANDIA in 2005/2007 showed increased body condition since release following their most recent recapture.

Closer examination of changes in the condition of Cape Sanctuary tuatara following release showed that $72 \%$ of the 18 individuals recaptured during the first postrelease survey in spring 2013 had experienced increases in mass. However, of the 11 tuatara captured during the first and final post-release surveys, $82 \%$ of these animals showed subsequent weight loss of 5-90 g over this 17 -month period. Overall, $56 \%$ of Cape Sanctuary individuals recaptured in autumn 2015 had lost mass in the range of 5-195 g since release, reaching final mean weights of $538 \mathrm{~g}$ for males and $324 \mathrm{~g}$ for females. These final mean masses are below the mean weights of the same individuals on release (males $=562 \mathrm{~g}$, females $=331 \mathrm{~g}$ ) and comparable to and below the mean masses observed in the source population on Stephens Island in the same season (mean males $=603 \mathrm{~g}$, mean females $=321 \mathrm{~g})$. Meanwhile, $91-100 \%$ of tuatara at the other three translocation sites showed mass gain in the range of 25-330 g. Individual percentage mass change between release and the final autumn 2015 survey ranged from increases of 14-90\% (mean $=25 \%$ ) for males and changes of $-5-43 \%$ (mean $=12 \%$ ) for females at Maungatautari, $10-35 \%$ $($ mean $=23 \%)$ for males and $15 \%$ and $18 \%($ mean $=17 \%)$ for the two females at Young Nicks Head, and $34-87 \%$ (mean $=69 \%$ ) for males and $19 \%$ and $42 \%$ (mean $=30 \%$ ) for the two females on Whangaokeno. Conversely, individual percentage mass change at Cape Sanctuary was in the range of $-33-29 \%($ mean $=-4 \%)$ for males and $-26-13 \%$ (mean $=-2 \%)$ for females. 

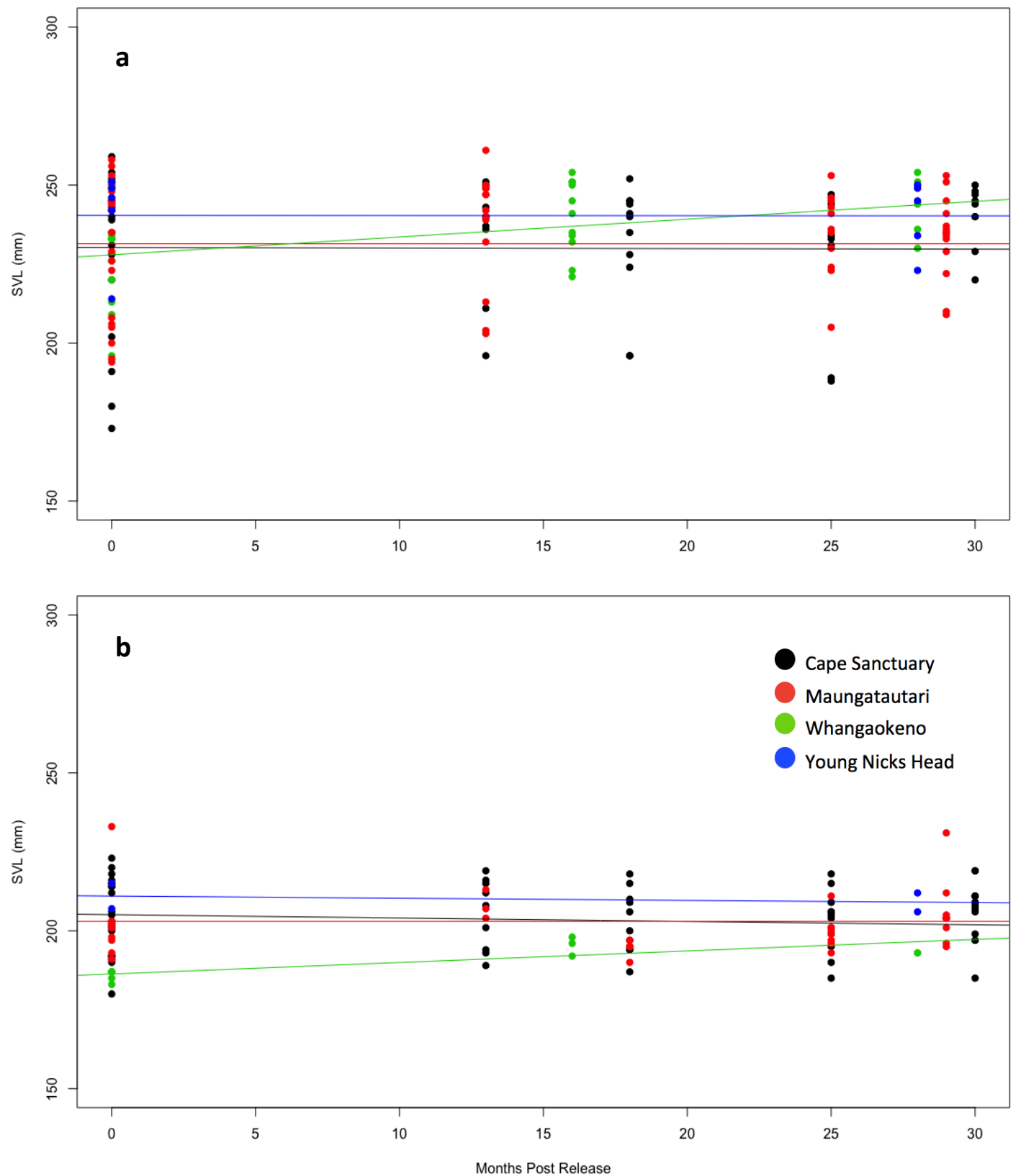

Figure 2.4 Individual SVL measurements for recaptured adult tuatara across post-release surveys (months post release) at the October 2012 translocation sites. Points show SVL measurements for individual animals. Lines show the estimated mean SVL growth rates. a) Male tuatara; b) Female tuatara. 

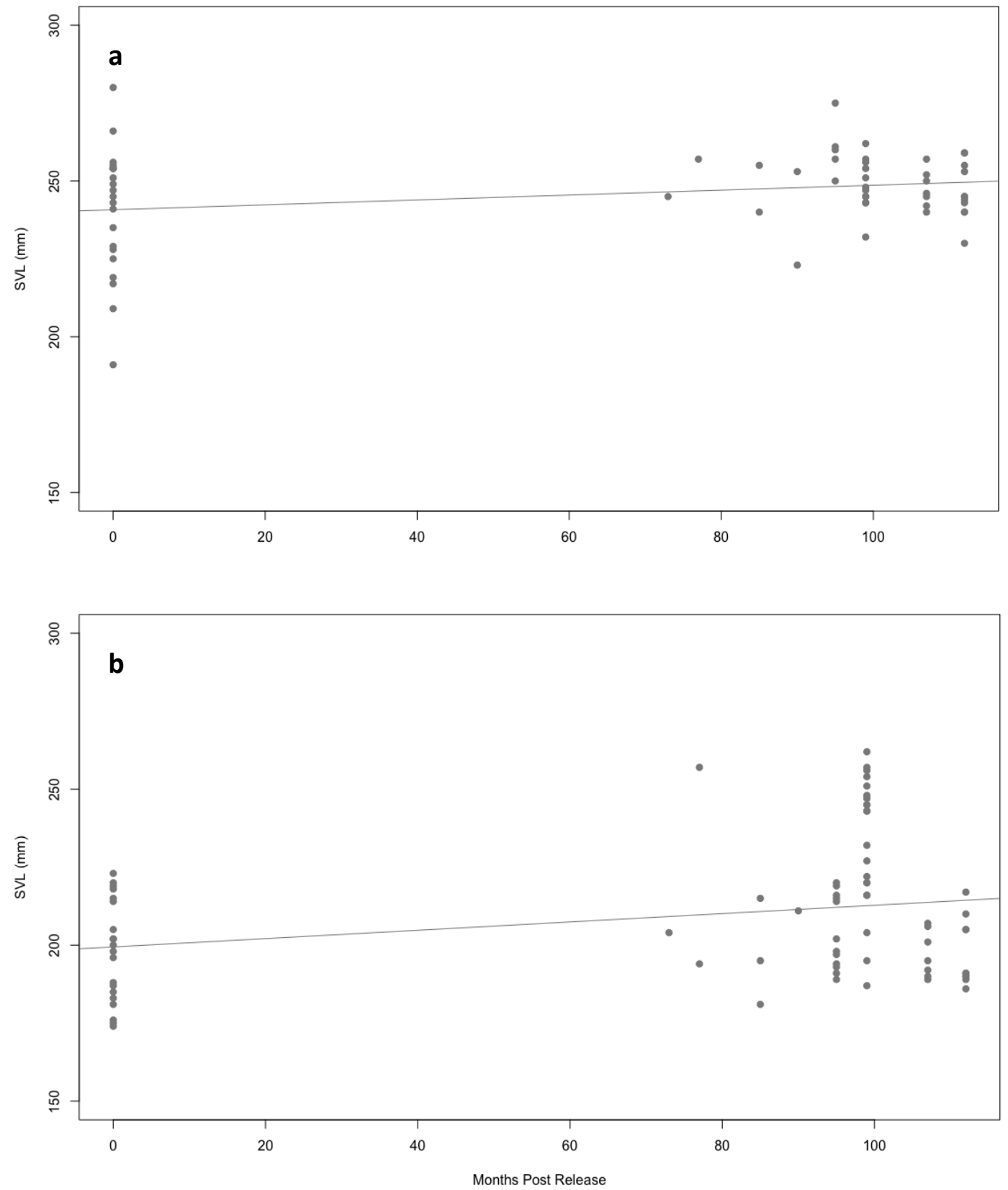

Figure 2.5 Individual SVL measurements for recaptured adult tuatara across post-release surveys (months post release) at ZEALANDIA. X-axis extends up to 113 months post-release. Lines show the estimated mean SVL growth rates. a) Male tuatara; b) Female tuatara. 

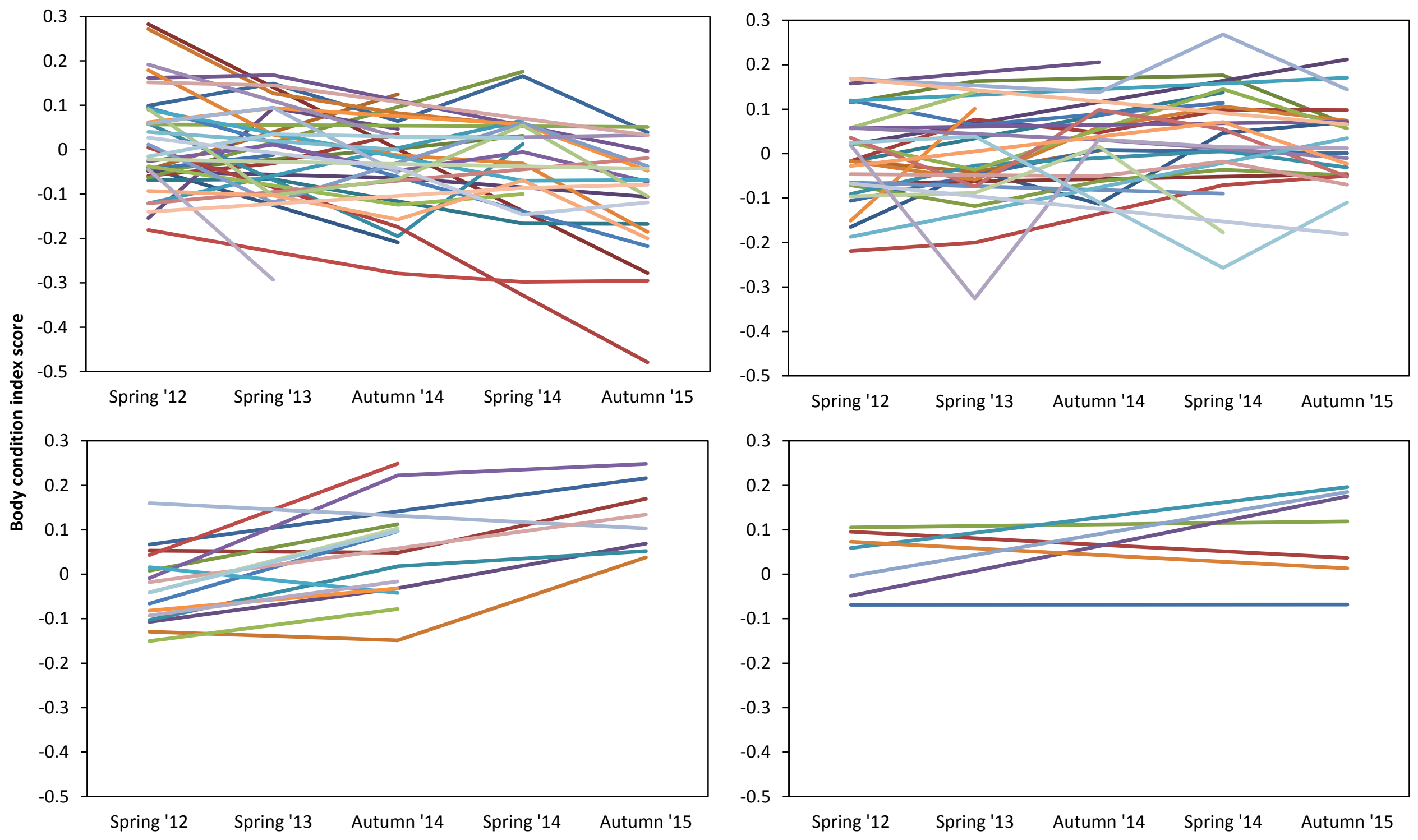

Figure 2.6 Individual $\mathrm{BCl}$ scores on release at translocation sites in spring 2012 and over the subsequent post-release surveys. Each line documents the $\mathrm{BCl}$ score of an individual adult tuatara. a) Cape Sanctuary $(n=37)$; b) Maungatautari $(n=31)$; c) Whangaokeno $(n=17)$; d) Young Nicks Head $(n=7)$. 
Juvenile tuatara at Cape Sanctuary and Young Nicks Head have also shown SVL growth and mass increases in the 23 to 35 months since release, with males showing, on average, greater mass gain, SVL increase (Table 2.3), and SVL growth rates than females in the same population. The SVL length at which tuatara are considered mature is in the range of $170-180 \mathrm{~mm}$ (Blanchard 2002). As such, based on the final juvenile SVL measurements, five (four males and one female; 25\%) of the Young Nicks Head juveniles and seven (five males and two females; 35\%) of the Cape Sanctuary juveniles released could now be considered adult animals, with SVLs at or above $180 \mathrm{~mm}$. Regarding SVL growth, juvenile females at Young Nicks Head grew significantly faster than juvenile females at Cape Sanctuary (estimate $=-0.44 \pm 0.10, \mathrm{t}=-4.28, \mathrm{p}<0.001$ ), with a mean estimated monthly growth rate of $1.40 \mathrm{~mm} \mathrm{month}^{-1}$ versus $0.96 \mathrm{~mm}$ month$^{-1}$ (or 16.80 $\mathrm{mm}$ year $^{-1}$ versus $11.52 \mathrm{~mm}$ year $\left.{ }^{-1}\right)$. There was no significant difference between growth rates of juvenile males at the two sites $(p>0.05)$, with a mean estimated monthly growth rate of $1.47 \mathrm{~mm} \mathrm{month}^{-1}$ in Young Nicks Head males and $1.45 \mathrm{~mm} \mathrm{month}{ }^{-1}$ in Cape Sanctuary males (or $17.64 \mathrm{~mm}_{\text {year }}^{-1}$ and $17.40 \mathrm{~mm}_{\text {year }}{ }^{-1}$ ). Mean juvenile SVLs were not significantly different between juvenile populations on release (females: $p>0.05$; males: $p>0.05)$.

Table 2.3 Mean juvenile male and female mass and SVL of tuatara at Cape Sanctuary and Young Nicks Head Sanctuary at release and at the final post-release survey. Mean changes from release to final survey are also shown. Data are based on individuals that were captured in the final survey. The final surveys at Young Nicks Head and Cape Sanctuary were done 23 and 35 months post-release respectively. The final Cape Sanctuary juvenile survey was conducted by Gibson et al. (2015). The sex of one Young Nicks Head individual could not be determined so its data has not been used. Multiple juveniles reached adult size over the course of the post-release surveys, as indicated by the final mean SVL $(\mathrm{mm})$.

\begin{tabular}{llcccccccc}
\hline \multicolumn{1}{c}{ Site } & Sex & $\begin{array}{c}\text { Release } \\
\text { mean } \\
\text { mass (g) }\end{array}$ & $\begin{array}{c}\text { Release } \\
\text { mean SVL } \\
(\mathbf{m m})\end{array}$ & $\begin{array}{c}\text { Final } \\
\text { mean } \\
\text { mass } \\
(\mathbf{g})\end{array}$ & $\begin{array}{c}\text { Final } \\
\text { mean } \\
\text { SVL } \\
(\mathbf{m m})\end{array}$ & $\begin{array}{c}\text { Mean } \\
\text { mass } \\
\text { change } \\
(\mathbf{g})\end{array}$ & $\begin{array}{c}\text { Mean } \\
\text { sVL } \\
\text { change } \\
(\mathbf{m m})\end{array}$ & $\begin{array}{c}\text { Mean } \\
\text { mass } \\
\text { change } \\
(\%)\end{array}$ & $\begin{array}{c}\text { Mean } \\
\text { SVL } \\
\text { change } \\
(\%)\end{array}$ \\
\hline $\begin{array}{l}\text { Cape } \\
\text { Sanctuary }\end{array}$ & M & 101.0 & 145.8 & 225.8 & 193.40 & 123.3 & 47.6 & 120 & 33 \\
$\begin{array}{l}\text { Young } \\
\text { Nicks }\end{array}$ & M & 87.3 & 139.3 & 184.3 & 174.75 & 93.1 & 35.5 & 102 & 25 \\
Head & F & 94.7 & 143.1 & 218.7 & 180.71 & 124.1 & 37.6 & 131 & 26 \\
\hline
\end{tabular}




\subsubsection{Evidence of reproduction}

No nests were found and no hatchling tuatara were observed during any of the surveys at Cape Sanctuary or Young Nicks Head Sanctuary. However, in March 2014, staff at Cape Sanctuary found three split eggshells within the tuatara enclosure, suggesting that tuatara have successfully hatched at the site (T. Ward-Smith, pers. comm.). Similarly, Ecoworks New Zealand Ltd. staff found and photographed four eggshells at Young Nicks Head Sanctuary in May 2015 (S. Sawyer, pers. comm.). In both cases eggshells appear to have been split open, suggesting that tuatara hatched successfully from them. No hatchling tuatara were observed during either of the two surveys at Whangaokeno. However a potentially successful nest was found on a rocky slope in February 2015. The nest contained several dried, split eggshells from which tuatara likely hatched. No hatchling tuatara or evidence of nests were found in spring 2013 or autumn 2014 at Maungatautari, though signs of digging, possibly for nest excavation, had been observed in the Tautari Wetland enclosure. In spring 2014 a nest was found on the main mountain with several swollen, potentially viable eggs inside. However, the nest consisted of a natural burrow, which had not been back-filled after laying so that eggs were clearly visible from the burrow entrance. Similar nests have been observed on Stephens Island, however whether or not the eggs will have been sufficiently insulated and protected from the elements to allow embryonic development is unknown (N. Nelson pers. comm.). In January 2015, while weeding the site, a member of the Maungatautari staff observed and photographed a hatchling tuatara in the Tautari Wetland enclosure. Another, or possibly the same, hatchling was later observed sheltering under a layer of corrugated sheeting that is used to provide shelter for invertebrates (M. Cook pers. comm.). In March 2015, a hatchling was also discovered on the main mountain inside a small burrow close to the pest-proof fence. The burrow had to be excavated and refilled as it could pose a pest invasion risk and the hatchling was found sheltering inside. Tuatara nests and eggs have been observed at ZEALANDIA each year since the 2005 release and a hatchling tuatara was observed for the first time in March 2009. Since then, there have been further observations of hatchlings as well as larger juveniles, particularly within the Research Area. In December 2014 a juvenile tuatara was photographed eating a hatchling, illustrating the presence of two sub-adult age cohorts within ZEALANDIA and further demonstrating that successful 
reproduction is occurring. Assuming that nests excavated after the 2005 release were successful and that hatchlings survived, it is feasible that there are now juveniles within ZEALANDIA approaching nine years of age.

\subsection{Discussion}

\subsubsection{Progress and short-term success}

\subsubsection{Adult tuatara}

A translocation cannot be accurately deemed a success until a population has been monitored for at least the time taken for an individual to reach sexual maturity (IUCN/SSC 1998; Germano \& Bishop 2008). Tuatara generally mature between 11 and 13 years of age and as the adult populations described here have only been monitored for $\sim 2.5$ years (28-31 months), this equates to only $20-23 \%$ of the time it takes to reach sexual maturity and $5 \%$ of the species generation interval ( $\sim 40-50$ years). Therefore, this study occurred over an insufficient period of time for an assessment of long-term population viability (Nelson et al. 2002b; Miller et al. 2010). In the meantime, I focussed on short-term measures of success to monitor the progress of the translocated populations. The results of the disease and parasite screening detailed in the monitoring methodology are reported elsewhere (Chapters Three and Four).

In the 31 months since release, cumulative recaptures of adults at Cape Sanctuary, Maungatautari and Whangaokeno have exceeded $30 \%$ and there is evidence of successful reproduction through the discovery of hatched eggs and/or the observation of hatchling tuatara at all translocation sites, including Young Nicks Head Sanctuary. However, as the age of tuatara cannot be determined, I was unable to confirm if these hatchlings were the result of post-translocation matings or from females that were translocated while gravid, with the exception of Whangaokeno, which received no gravid females.

By all short-term measures (i.e. $>30 \%$ survival, growth of founders, and evidence of reproduction) the translocations from Stephens Island to Whangaokeno can be considered as successful in the short term and to be progressing favourably. Similarly, although the Maungatautari population has shown negligible mean SVL growth rates, body condition, SVL and mass have increased for the majority of translocated individuals, 
and there has been a high recapture rate and signs of reproduction, which suggest favourable progress in the short term. In contrast, the adult tuatara at Young Nicks Head Sanctuary appear to be progressing well in terms of evidence of reproduction; however, SVL growth was negligible, and as access to this population was limited, the recapture numbers were low so I have comparatively less data on this translocation's progress. Nonetheless, this population has demonstrated overall increases in body condition, many have shown SVL increases, and all recaptured individuals gained weight post-release, which bodes well in the short term. The mass and SVL gains observed in several of the adult populations post-release supports previous work by Nelson et al. (2002b), which found post-translocation growth in adult tuatara that had not grown in the seven years prior to translocation, indicating an indeterminate growth pattern in tuatara. Conversely, the adult tuatara at Cape Sanctuary appear to be progressing favourably regarding founder survival and reproduction, but a negligible growth rate, declining overall body condition and weight loss in the majority of the recaptured population is cause for concern.

When compared to data collected over a similar time frame from the control population at ZEALANDIA (animals translocated in 2005 and surveyed for one year postrelease; McKenzie 2007) and other previous tuatara translocations, some of the translocations studied here are showing comparably positive progress. Since the 1995 and 1998 translocations of tuatara to Titi Island and Matiu/Somes Island, 63\% and 60\% (respectively) of founders have been recaptured, all founders have increased in size, with a mean mass increase of $41 \%$ by 2000 in Titi Island adults, and evidence of reproduction has been observed (Nelson 1998; Nelson et al. 2002b; Miller et al. 2010). SVL growth in the range of 18-39 $\mathrm{mm}$ was also observed in the adult Titi Island population five years post-release (Nelson et al. 2002) and while few of the tuatara recaptured in this study had such substantial SVL increases, this difference is likely attributable to the greater amount of time elapsed between release and the final surveys reported on here ( 2.5 years) and the final Titi Island survey (5 years). Two separate translocations to Wakatere-papanui Island in 2003 and 2004, which saw 432 wild-caught and head-started animals released, have seen recapture rates of $25 \%$ of the wild-caught founders and $6 \%$ of head-started juveniles during subsequent surveys. All recaptured adult animals had grown, with adult males showing a mean net mass increase of $43 \%$ three years after release (Nelson et al. 
2008). Data collected from ZEALANDIA in the course of this study also show that, since the 2006 survey that documented mean mass gains of $500 \mathrm{~g}$ for males, $49 \mathrm{~g}$ for females, and small SVL increases (McKenzie 2007), individuals in the population have continued to grow and there is now widespread evidence of reproduction at the site, with a range of sub-adult age cohorts repeatedly observed. If the populations translocated in 2012 continue to show similar progress then this would bode well for longer-term translocation success.

While the recaptures at Whangaokeno and Young Nicks Head Sanctuary are comparatively lower than other sites (39\% and 17\% respectively), placing Young Nicks Head Sanctuary below the $30 \%$ recapture threshold criterion, it is important to acknowledge the limitations that could have contributed to these low numbers. Firstly, these sites cover large areas and have consequently lower population densities than Cape Sanctuary and Maungatautari. Equally, the smaller fenced enclosures within the large Cape Sanctuary and Maungatautari Tautari Wetland sites were easier to systematically search, whereas Young Nicks Head is a much larger, fenced site and Whangaokeno is both large and unfenced, affording tuatara greater opportunities for concealment or evasion and making systematic searches more difficult. Secondly, the nature of opportunistic monitoring means that overall search effort was lower at Whangaokeno and Young Nicks Head Sanctuary, with fewer "person nights" (an approximate measure of search effort) spent on surveys at these sites. However, while recaptures may have been low nondetection does not equate to mortality; therefore, recaptures are not necessarily indicative of the number of surviving individuals (Nelson et al. 2002b). Tuatara are cryptic, burrow-dwelling reptiles that are most active at night. Combined with low population densities, complex vegetation and inaccessible habitat (e.g. steep slopes, dense vegetation), these factors can hinder access by surveyors, conceal tuatara, and reduce detection probability (Nelson et al. 2002b; Miller et al. 2010). As such, attributing low recapture numbers to mortality, difficulty of detection, or limitations arising from habitat complexity can be problematic (Towns \& Ferreira 2001). Such complications have been encountered following translocations of other reptiles including skinks, geckos, snakes and crocodilians, and a review by Ewen et al. (2014) of 40 herpetofauna translocation case-studies found difficulties in post-release monitoring to be one of the major problems 
faced (Towns \& Ferreira 2001; Nelson et al. 2008; Tolson et al. 2008; Wu \& Jiang 2008; Baling et al. 2010; Miller et al. 2010; van Winkel et al. 2010; Fitzgerald et al. 2015).

More optimistically, the increasing cumulative recapture numbers for Whangaokeno and Maungatautari indicate that new founders are recaptured with each successive survey, as was also the case at ZEALANDIA, and suggests that future surveys could uncover animals not seen since translocation. In contrast, cumulative recaptures at Cape Sanctuary plateaued at 95\% from spring 2014 onwards, suggesting that the maximum number of possible recaptures may have been reached or that an individual could have climbed out of the enclosure and dispersed into the wider seabird cell. Adults have since been observed outside of the tuatara enclosure, lending support to this possibility (T. Ward-Smith, pers. comm.). Consequently, founder survival at all translocation sites is likely higher than estimated by recapture rates and should be taken as minimum number alive, rather than indicative of number surviving. Accounting for this, it can be inferred that founder survival at Young Nicks Head Sanctuary likely exceeds $17 \%$ and, in combination with evidence of improved body condition, mass gain, and reproduction, suggests this population is also progressing favourably in the short term.

That recaptures have been found to increase cumulatively over time serves to emphasise the need for multiple surveys in order to obtain reliable measures of founder survival and short-term translocation success (Miller et al. 2010). It also calls into question the suitability of short-term success criteria like the " $30 \%$ within three years" recapture threshold. As such, it is important that methodologies, monitoring objectives, and success indicators are defined and adhered to if reliable, consistent and useful feedback on a translocation's progress is to be obtained (Ewen et al. 2014). However, restrictions to site access (either due to permission limitations or physical access) can limit the number of post-release surveys that can be conducted within a three year timeframe and negatively impact survival estimates, risking the categorisation of a potentially successful translocation as a failure, or vice versa, which could jeopardise the efficiency of, or investment in, further management and monitoring efforts (Seddon 1999; Miller et al. 2014). While there may be pressure to provide prompt feedback on the progress of a translocation, which can be useful in the identification of urgent issues, it is vital that progress reports acknowledge the influence of limitations like monitoring restrictions and time since release when conveying short-term progress and consider the appropriateness 
of success criteria on a case-by-case basis. Equally, while authors may be disinclined to report failed translocations, the publication of failures and uncertain outcomes are vital to the development of translocations as a conservation tool (Germano \& Bishop 2008; Miller et al. 2014).

Due to the small sample sizes obtained from the Whangaokeno and Young Nicks Head Sanctuary populations in the final post-release survey I was unable to investigate potential relationships between post-release growth and site-specific factors like population density and air temperature. Therefore, possible explanations for the differences observed between sites are entirely qualitative.

One explanation for the greater growth rates exhibited by Whangaokeno tuatara is their comparative size on release. Whangaokeno individuals had smaller mean SVLS when translocated and were therefore at an earlier point on the von Bertalanffy growth curve, at which the growth rate is higher (Dawbin 1982). However, growth rates were calculated only from individuals that were recaptured post-release, between which there were no significant differences in mean SVLS by population (with the exception that Whangaokeno females were smaller than ZEALANDIA females). This can be explained by the finding that animals with larger SVLS on release were more likely to be recaptured on Whangaokeno. This bias is not likely due to competitive exclusion influencing differences in survival rates (given the low population density and substantial growth/BCl increases seen in this population), but could perhaps be attributed to possible size-dependent differences in the use of the habitats I searched, or differences in detection avoidance/escape ability, though this explanation is speculative. This similarity in the mean SVL sizes of recaptured tuatara across sites contradicts the theory that earlier placement on the growth curve is responsible for the higher growth rates, suggesting that site specific or environmental factors may have played a role.

Body size and growth in reptiles is influenced by environmental factors, such as resource availability and air temperature, which can vary with population density and the size and location of a site (Nelson et al. 2002b; Angilletta et al. 2004; Angilletta 2009; Cree 2014). Whangaokeno's lower latitude means that it has a generally warmer climate than the other three translocation sites, which could allow tuatara to attain warmer body temperatures, potentially facilitating the faster growth rates observed in tuatara postrelease. Equally, while Whangaokeno is a relatively small island it supports a variety of 
habitats (see Appendix 2, Figure 2.1) that could host a diversity of prey species (Cree 2014), the competition for which would likely be influenced by population density. As such, translocation of individuals to a lower density population and the consequent competitive release could also have facilitated the greater growth observed on Whangaokeno. Relief from density-dependent competition for resources was also considered to be a potential driving factor behind the large mass increases seen in adult tuatara moved from the more densely populated North Brother Island to Titi Island (Nelson et al. 2002b). Density-dependent growth has been documented in multiple taxa, including reptiles (Wilbur 1977; Gibson \& Hamilton 1984; Petranka \& Sih 1986; Choquenot 1991; Kasuya 1991; Massot et al. 1992; Grant \& Imre 2005) though surveys on factors affecting density-dependent growth (e.g. invertebrate prey abundance) were not conducted in this study. As such, it is feasible that individual or combined effects of a warmer climate, lower population density, and consequently reduced competition could have led to the significantly greater growth observed in this population.

Continued monitoring of SVL growth in the Whangaokeno population will also be useful in informing the cause of the linear relationship between tuatara SVL and island size, with larger tuatara found on larger islands and vice versa (Cree 2014). It has been hypothesised that this could be due to variations in habitat among islands, resource abundance and stability, or the presence/absence of introduced predators, but as yet there is no clear answer (Cree 2014). Given this relationship it would be expected that Whangaokeno tuatara will reach shorter maximum SVLs than tuatara on larger islands like Tawhiti Rahi (a 151.5 ha island in the Poor Knights group), where adult males can reach SVLs of up to $302 \mathrm{~mm}$ (Cree 2014). Assessment of the SVL lengths at which Whangaokeno tuatara stop growing will be useful in informing the drivers of this relationship.

Consistent with the hypothesis that environmental and/or other site specific factors might have influenced post-release growth, smaller mean increases in mass and lower/negligible growth rates were seen in the Cape Sanctuary and Maungatautari populations (specifically relevant to the Maungatautari Tautari Wetland population, where the majority of searches were focussed and recaptures were made), which host comparatively higher population densities and have, on average, cooler climates than Young Nicks Head Sanctuary or Whangaokeno. Given that Cape Sanctuary had a similar population density to Maungatautari, the low growth rates and declining weights 
observed in multiple individuals at the former site could be explained by limited resource availability and high intraspecific competition. The density of tuatara within the Cape Sanctuary adult enclosure is one of the highest of the translocated populations (200 per hectare) and the initial post-release increases and subsequent declines observed in mass, combined with data from the final survey showing that $56 \%$ of recaptured founders weighed less than they did on release, could suggest that an initially plentiful supply of prey within the enclosure had dwindled, leaving tuatara in competition for limited resources. A more site-specific issue such as periods of drought over the summer season could also have influenced humidity/water availability, invertebrate prey populations and, consequently, tuatara physical condition, especially as tuatara lose water, which is required to maintain body weight, through their skin (Hill 1982). As such, I suggest that the negligible growth rates, declining body conditions and weight loss observed in the majority of the Cape Sanctuary adult population could be attributed to cumulative effects of a drier, more drought-prone climate, high population density, lower resource availability, and consequent competition between conspecifics. Density-dependent responses to competition for limited resources were also thought to be responsible for significant declines in body condition observed in the Stephens Island tuatara population between 1949 and 2003 (Moore et al. 2007). In order to reduce population density and increase resource availability a management recommendation was made that the fence surrounding the adult tuatara enclosure at Cape Sanctuary be removed or modified to enable dispersal into the wider Seabird Cell and help relieve intraspecific competition.

\subsubsection{Juvenile tuatara}

Regarding the head-started juveniles translocated to Cape Sanctuary and Young Nicks Head Sanctuary, both translocated populations have shown high survivorship of at least $65 \%$ and $100 \%$ respectively, as well increases in mass and SVL. Growth rates for these individuals were comparable between populations, though higher growth rates were observed in Young Nicks Head juvenile females when compared to Cape Sanctuary juvenile females, and were higher for males than females at both sites. Several individuals are now within the adult size range (170-180 mm and above; Blanchard 2002), though they are still below the estimated age at which tuatara are thought to reach sexual 
maturity. The growth rates recorded exceed the annual mean growth estimates reported for wild Stephens Island juveniles (7.6 mm; Mello et al. 2013) and were also comparable to those reported by Jarvie et al. (2015) for headstarted juveniles translocated to Orokonui Ecosanctuary in spring 2012 (means 0.05 and $0.04 \mathrm{~mm} \mathrm{day}^{-1}$ or an estimated 18.3 and $14.6 \mathrm{~mm}$ year ${ }^{-1}$ ), but appear substantially slower than wild-caught juveniles released at the same location (mean $0.10 \mathrm{~mm}$ day $^{-1}$ or an estimated $36.5 \mathrm{~mm}$ year ${ }^{-1}$ ). The authors suggest that the differences between translocated wild and translocated head started juveniles might be due to thermal differences between the differently reared groups. They recorded warmer body temperatures in the wild-caught juveniles, which may have cause faster growth rates, or suggest that the wild-caught juveniles may have been more active, persistent foragers owing to their warmer body temperatures and lack of experience with captive supplementary feeding (Jarvie et al. 2015). However, as I did not record juvenile body temperatures I cannot assess the relevance of these hypotheses to the growth observed in the head started juveniles studied here. Post-release recapture rates were also similar between the populations studied here and the juveniles released at Orokonui Ecosanctuary (at least 67-72\%; Jarvie et al. 2015), and higher than other translocations involving head-started juvenile tuatara (Nelson et al. 2002b; Nelson et al. 2008), though the smaller, more easily searched enclosures at Cape Sanctuary and Young Nicks Head Sanctuary likely aided in the recapture of higher numbers of juveniles at these sites.

\subsubsection{Addressing common causes of success/failure}

General and taxa-specific reviews have highlighted key factors that can predict the success or failure of a translocation. One of the most significant determinants of translocation success is the size of the founder group (Griffith et al. 1989; Wolf et al. 1996; Fischer \& Lindenmayer 2000; Germano \& Bishop 2008). However, when translocating a protected species, small founding populations have historically been an unavoidable necessity to prevent potential damage to vulnerable source populations, and it has not always been possible to translocate large numbers of tuatara (Towns \& Ferreira 2001; Nelson et al. 2002b). Consequently, low numbers of wild adult tuatara are typically translocated and the new populations have been supplemented with higher numbers of 
juveniles, with the exception of the 2005 and 2007 translocations to ZEALANDIA, in which a total of 200 adults were translocated (Gaze 2001; Nelson et al. 2002b). Small founding populations can be a particular problem for a slowly reproducing species like the tuatara as this can further reduce an already slow population growth rate, a potential predictor of failure in reptile translocations (Towns \& Ferreira 2001).

In the case of the 2012 translocations, founding populations of 40-50 tuatara were released at each recipient site, totalling 176 adult animals removed from the source population. Two sites also received a supplementation of head-started juveniles to help increase the size of the founding population, however these animals will not be able to breed until approximately 11-13 years of age and so any reproductive contribution will not be seen until 8-10 years post-release. Considering that the population on Stephens Island is an estimated $30-50,000$ animals, it is unlikely that removal of $\sim 0.7 \%$ of the population will have significantly impacted the remaining tuatara and could instead relieve intraspecific competition between individuals in the vicinity of the capture locations. The use of small founder groups seems counterintuitive to the findings of various reviews that the chance of a successful translocation increases with founder group size (Griffith et al. 1989; Wolf et al. 1996; Fischer \& Lindenmayer 2000). However, Germano and Bishop (2008) found that this was not the case for reptile translocations and it has been suggested that smaller founding populations may be acceptable for species with extreme longevity as their long lifespans and lower variability in lifetime reproductive success mean that individuals will likely survive to breed again even if there is a poor reproductive season (Armstrong \& McLean 1995). Equally, some of the smaller enclosures into which tuatara were released in 2012 would not have been suitable for larger founding populations (i.e. the Maungatautari Tautari Wetland and Cape Sanctuary adult enclosures). As such, and supported by evidence of successful nesting and hatching at each translocation site, there is no evidence as yet that translocated populations are suffering from negative demographic effects due to small population size. However, small populations will always be vulnerable to negative genetic effects (e.g. inbreeding, loss of variation) and stochastic effects such as extreme weather, disease outbreaks and pest incursions (Towns \& Ferreira 2001; Miller et al. 2009).

Other predictors of translocation success are the release of animals into high quality, protected habitat at physiologically suitable sites within the species' historical 
range at which the initial cause of decline has been addressed (Griffith et al. 1989; Wolf et al. 1996; Fischer \& Lindenmayer 2000). Whangaokeno, Young Nicks Head Sanctuary, Cape Sanctuary, Maungatautari and ZEALANDIA are all within the historical range of tuatara, have all been extensively replanted with native flora or retained areas of native vegetation, are largely free of invasive mammals and are all protected from reinvasion by pest-proof fences or an expanse of ocean. Such barriers also serve to prevent the dispersal or migration of translocated individuals away from the release sites, another common cause of herpetofauna translocation failure (Germano \& Bishop 2008). Release at a physiologically suitable site within a species' active range is particularly relevant for coldadapted and ectothermic species like tuatara and it is important that animals have a range of thermal conditions available (Towns \& Ferreira 2001). Tuatara demonstrate a reasonably broad, if low, active body temperature range of $5-30^{\circ} \mathrm{C}$; the sites to which they have been translocated all experience temperatures favourable to behaviours like basking and nesting, and artificial refuges offer shelter if needed (Bogert 1953; Wilson \& Lee 1970; Barwick 1982; Cree 2014). A primary concern and aim of this study was to assess the impact that warmer climates may have on tuatara populations and translocation success with a view to assessing how tuatara may fare in the face of anthropogenic climate change. As several tuatara populations have so far demonstrated high survival rates, growth, and some evidence of reproduction at sites that can exceed Stephens Island mean temperatures by $2-4^{\circ} \mathrm{C}$, this suggests that so far these warmer climates have not negatively affected the establishment or survival of translocated individuals and that tuatara may be capable of tolerating the warmer air temperatures predicted by the $2100 \mathrm{~s}$ under climate change. However, it is important to highlight that the declining condition of individuals in the Cape Sanctuary population could be attributed to environmental factors e.g. drought, the incidence of which is predicted to increase substantially under climate change scenarios (IPCC 2014; Reisinger et al. 2014). As such, it may not be air temperatures that directly affect survival of cold-adapted tuatara as climate change progresses, but their effect on associated biotic factors and consequent impacts on aspects of habitat, disease and prey populations. 


\subsubsection{Conclusions and conservation implications}

In summary, the majority of factors commonly associated with the success and failure of translocations have been addressed in the short term at each of the 2012 translocation sites. Similar steps have been taken and programmes put in place as for the 2005 and 2007 translocations to ZEALANDIA. By all short-term success criteria, the ZEALANDIA population is progressing very favourably and should see maturation and breeding of first generation ZEALANDIA tuatara within the next few years. The short-term progress at each of the 2012 translocation sites is similar to and in some cases exceeds previous tuatara translocations in terms of both founder recaptures and mass increase, with recaptures of up to $95 \%$ of founder populations and weight gains of up to $87 \%$ in the two and a half years since release.

This study uses data collected from multiple translocated populations surveyed repeatedly over 2.5 years post-release and provides the first evaluation of short-term success for tuatara translocated to sites at latitudes beyond the source island's ecological region. Results indicate that the tuatara, a slowly reproducing, cold-adapted reptile, has demonstrated high survival rates as well as growth and reproduction after translocation to distant locations with generally warmer climates than those to which they are acclimatised, with comparatively greater growth observed at the warmer, less densely populated sites. The success so far suggests that the Stephens Island tuatara population may be suitable for further translocations to sites around New Zealand and bodes well for tuatara survival when facing air temperature increases of up to $5^{\circ} \mathrm{C}$, but there remain concerns regarding their ability to cope with associated abiotic factors that are expected to increase in frequency e.g. drought. Given the short-term success of translocations of juvenile tuatara to cooler locations (i.e. Orokonui Ecosanctuary; Jarvie et al. 2015) and the suitability of these higher latitude sites for successful nest incubation (Besson et al. 2012), additional translocations to more southerly sites would also be a conservation strategy worth further consideration. Assuming that environmental conditions at release sites remain favourable, pest-control programmes are maintained and steps are taken to allow dispersal of animals at Cape Sanctuary, it is feasible that the populations at Whangaokeno, Young Nicks Head Sanctuary, Cape Sanctuary and Maungatautari could progress as favourably as the population at ZEALANDIA. However, owing to the extreme longevity, 
late sexual maturation and slow reproduction of tuatara, decades of monitoring will be required to confirm the establishment of viable, self-sustaining populations at these sites (Towns et al. 2001; Nelson et al. 2002b). 


\section{Chapter Three}

The association between translocation (and associated climate shifts) and the prevalence of the gastrointestinal colonisers, Campylobacter spp. and Salmonella spp. in tuatara (Sphenodon punctatus)

\subsection{Introduction}

The interaction between environmental temperature and disease is an important, but often overlooked, aspect of reptile conservation in the face of anthropogenic climate change. Future scenarios of global air temperatures predict increases of $0.3-0.7^{\circ} \mathrm{C}$ by 2035 and up to $4.8^{\circ} \mathrm{C}$ by 2100 (IPCC 2014). A principal concern is that reptiles may become more susceptible to disease as the climate warms owing to changes in disease ecology dynamics, including the possibility that pathogens may show increased growth rates, survival and transmission capabilities. In an ectothermic species, changes in climate may also result in changes in host susceptibility, with some pathogens likely to benefit from range expansion, whilst the spread of diseases requiring cool, humid environments may be restricted (Harvell et al. 2002). Warming temperatures have already been linked to an increase in reports of disease in marine fauna; temperature-dependent bacterial pathogenicity has been observed in cnidarians and outbreaks of infectious diseases responsible for global amphibian declines and extinctions may be driven by climate change (Daszak et al. 1999; Romano et al. 2000; Ward \& Lafferty 2004; Pounds et al. 2006; Bally \& Garrabou 2007). Understanding the impact of a shift in climate on reptile health is a high conservation priority, especially for cold-adapted reptiles that are vulnerable to increasing environmental temperatures. However, to date there has been little work on how climate change might impact the prevalence of both commensal and potentially pathogenic bacteria in such species.

Pathogenic bacteria like Salmonella are a risk to wildlife as they can reduce individual fitness and population viability through impaired reproductive success, increased vulnerability to predation and large-scale mortalities (Anderson \& May 1979; Scott 1988; Janssens \& Stoks 2014). Salmonella spp. are gram-negative and potentially fatal bacteria present in the environment and in multiple animal taxa including mammals, birds and reptiles (Rabsch et al. 2015). Although relatively common as gastrointestinal 
colonisers in reptiles worldwide (with population prevalence sometimes in excess of $90 \%$ ), a considerably lower true prevalence of $2.3-8.8 \%$ has been found in New Zealand reptiles (Hoff \& White 1977; Otokunefor et al. 2003; Chambers \& Hulse 2006; Middleton et al. 2010; Franco et al. 2011; Bull et al. 2012; Baling et al. 2013a). Routes of infection are usually via faecally contaminated food and water, soil or contact with infected organisms and bacteria are able to survive in water, soil, and faeces for long periods of time outside of a host, offering numerous opportunities for transmission (Mermin et al. 2004; JohnsonDelaney \& Mader 2006; Haraga et al. 2008; Broz et al. 2012; Bull et al. 2012). However, in reptiles infection alone is not a health risk as many Salmonella serovars are likely commensal organisms and Salmonella spp. are regularly isolated from the intestinal tracts of clinically normal, asymptomatic reptiles (Chiodini \& Sundberg 1981; Bäumler et al. 1998; Mitchell \& Shane 2001; Barrow et al. 2011). However, asymptomatic animals can be considered bacterial reservoirs that will intermittently shed the bacteria and facilitate transmission to uninfected individuals in which they could cause systemic illness (Pfleger et al. 2003; Gray 2011; Middleton et al. 2014).

The systemic spread of Salmonella spp. from the intestinal tract is usually inhibited by a combination of factors (e.g. mucus layers, antimicrobial peptides, volatile organic acids), but environmental and anthropogenic stressors like heat, captivity and/or illness can suppress the immune system and allow pathogenic bacteria to cause disease (Cambre et al. 1980; Carey 1993; Mahida et al. 1997; Alford \& Richards 1999; Gibbons et al. 2000; Pasmans et al. 2003; Franco et al. 2011; Broz et al. 2012). Salmonellosis, the disease caused by Salmonella spp. infection, can present in reptiles with symptoms including reproductive failure and disease, abscessation, septicaemia, pneumonia, inflammation and death. An increase in Salmonella prevalence in humans and birds in past decades has resulted in its classification as an emerging infectious disease (Daszak et al. 2000; Dobson \& Foufopoulos 2001; Alley et al. 2002; Johnson-Delaney \& Mader 2006; Ewen et al. 2007; van Andel et al. 2014). In New Zealand, Salmonella spp. have been identified in native and introduced reptiles, birds and mammals and have been implicated in the deaths of hihi (Notiomystis cincta), masked lapwing (Vanellus miles novaehollandiae), tuatara (Sphenodon punctatus), saddleback (Philesturnus carunculatus), New Zealand sea lions (Phocarctos hookeri) and livestock (McInnes 1971; Clark 2001; Alley et al. 2002; Clark et 
al. 2002; Castinel et al. 2007; Middleton et al. 2010, 2014; Kikillus et al. 2011; van Andel et al. 2014).

Campylobacter spp. are enteric, gram-negative bacteria that are able to survive temporarily outside of the host, enabling transmission via contaminated food, water and soil (Tu et al. 2004; Bull et al. 2006; Inglis et al. 2010; Jäderlund et al. 2010). Several species are considered to be emerging pathogens, with campylobacteriosis judged a major public health problem in New Zealand (Mullner et al. 2009; Man 2011). Campylobacter spp. have been isolated from birds, mammals and reptiles and, while they can be pathogenic, are considered to be commensal in the intestinal tract of many species and have been isolated from clinically normal reptiles. There are no reports of Campylobacter spp. causing disease in reptiles (Harvey \& Greenwood 1985; Man 2011; Wang et al. 2013; Giacomelli \& Piccirillo 2014; Gilbert et al. 2014). Whilst the prevalence of Campylobacter spp. in wild birds, livestock and waterways in New Zealand has been widely studied due to their relevance as reservoirs for human infection, there is little in the literature regarding prevalence in native reptiles (Ikram et al. 1994; Eberhart-Phillips et al. 1997; Savill et al. 2001; French et al. 2009; Sears et al. 2011).

The tuatara is a New Zealand endemic reptile and the sole extant representative of the Order Rhynchocephalia (Cree 2014). Tuatara are medium-sized reptiles with long lifespans and a cold-adapted active body temperature range of $5-30^{\circ} \mathrm{C}$ (Bogert 1953 ; Barwick 1982; Cree 1994; Thompson \& Daugherty 1998; Besson \& Cree 2010), although tuatara have been observed to suffer heat stress and death at air temperatures above $25^{\circ} \mathrm{C}$ (Blanchard 2002). Holocene subfossil records indicate that tuatara were once widespread throughout New Zealand (Chapter One, Figure 1.1), but with the introduction of mammalian predators they were restricted to predator-free offshore islands (Cree \& Butler 1993; Anderson 1996; Holdaway \& Worthy 1997). Conservation translocations have since served to extend their current range, but tuatara are still considered to be conservation dependent, range restricted and relict (Miller et al. 2012; Hitchmough et al. 2016; Cree 2014). Their cold adapted lifestyle places tuatara at risk from rising air temperatures, but also makes them an ideal species for studying the early effects of climate warming on reptiles and on reptilian bacterial communities (Nelson et al. 2002b; Mitchell et al. 2008). 
Despite close association with species known to excrete Salmonella spp. (i.e. seabirds and skinks) and presence of the bacteria in soil around burrows, there are no reports of Salmonella spp. isolation from live tuatara, although Salmonella enterica subspecies houtenae has been isolated from a deceased adult female (Tizard 2004; Gartrell et al. 2007; Middleton et al. 2010, 2014; van Andel et al. 2014). Equally, previous work to detect Campylobacter spp. in tuatara has been equivocal (B. Gartrell, A. Midwinter pers. comm.) so prevalence in tuatara has not been established. Prevalence of Salmonella spp. in the environment and their long life span outside of hosts makes lack of exposure an unlikely explanation, and alternative hypotheses suggest that tuatara could be innately resistant, or the species' low body temperatures might inhibit bacterial proliferation (Johnson-Delaney \& Mader 2006; Gartrell et al. 2007; Middleton et al. 2014, 2015). The stress-associated intermittence with which bacteria are shed from the intestinal tract can also produce false negative results, making detection problematic (Gartrell et al. 2007).

Salmonella spp. have a growth temperature range of $5-45^{\circ} \mathrm{C}$, with optimal growth occurring between $35^{\circ} \mathrm{C}$ and $37^{\circ} \mathrm{C}$ (Tajkarimi 2007). Conversely, tuatara have an active temperature range of $5-30^{\circ} \mathrm{C}$ and an upper lethal limit of $\sim 34^{\circ} \mathrm{C}$, below the optimum temperature range for Salmonella spp. growth (Heatwole 1982). As other cold-adapted New Zealand reptiles have been found to carry a range of Salmonella serovars it is unlikely that suboptimal temperatures alone would make tuatara resistant to Salmonella spp. colonisation (Middleton et al. 2010). The identification of Salmonella-responsive antibodies in tuatara suggests that the inability to detect Salmonella spp. may be partially due to an adaptive immune response (Middleton et al. 2015). Supposing that the low body temperatures maintained on Stephens Island contribute to the inhibition of Salmonella spp. colonisation, then warmer environmental temperatures could enable tuatara to maintain higher body temperatures for longer periods of time, which could be more favourable to and enable bacterial proliferation. Increasing environmental temperatures are also pertinent to thermal stress in cold-adapted ectotherms; if a pathogenic bacterium's thermal optimum exceeds that of its host, then warmer temperatures could impair the host's immune response while simultaneously improving conditions for the pathogen (Harvell et al. 2002). There are also disease risks inherent to translocation, with translocated animals not only at risk of exposure to novel species and pathogens, but also 
potential carriers of pathogens that they may introduce to naïve local populations (Jacobson 1993; Tompkins et al. 2011; Almberg et al. 2012). Stress caused by captivity, handling, transport and re-establishment in a new environment can also cause immunosuppression, making individuals more susceptible to infection on release (Dickens et al. 2010; Kock et al. 2010).

This is the first comparative study to look at the relationship between air temperature and tuatara disease ecology and utilises several unprecedented large-scale conservation translocations to sites around New Zealand's North Island to assess the association between warmer climates and translocation on Salmonella spp. and Campylobacter spp. prevalence in tuatara. The comparatively warmer climates of the translocation sites studied, which are on average $2-4^{\circ} \mathrm{C}$ warmer than the source site during summer months, provide surrogates for temporal climate change and enable an assessment of how bacterial prevalence may be affected as the climate warms (Chapter Two, Figure 2.1). Whilst commensal organisms are usually of little health concern to the reptiles, if warmer air temperatures enable the proliferation of pathogenic bacteria then climate change could trigger increased incidence of disease and threaten the survival of wild tuatara populations. Salmonella spp. and Campylobacter spp. are also zoonotic organisms and contact with reptiles has been cited as a source of bacterial infection in humans (Ackman et al. 1995; Mermin et al. 2004; Coburn et al. 2007; Gray 2011; Marin et al. 2013; Giacomelli \& Piccirillo 2014). As such, prevalence of these bacteria in tuatara is also of importance to human health, specifically for conservation workers involved in tuatara translocation and monitoring. Here, in accordance with the goals of the Tuatara Recovery Plan (2001), I aim to investigate the existence of both commensal and pathogenic microorganisms and how prevalence might be influenced by translocation to warmer climates.

\subsection{Methods}

\subsubsection{Founder and translocation sites}

In October 2012176 adult tuatara were translocated from Takapourewa/Stephens Island in the Cook Strait to four sites around New Zealand's North Island: Maungatautari 
Ecological Reserve, Whangaokeno/East Island, Cape Sanctuary and Young Nicks Head Sanctuary (Chapter One, Figure 1.1). These translocations were designed to benefit conservation goals and all animals were released at protected, pest-free sites within their historical range (Worthy \& Holdaway 2002; Wood 2009; IUCN/SSC 2013). Stephens Island, the source site, is a pest-free, 150 ha offshore island nature reserve in the Marlborough Sounds and is a site of high conservation significance (East et al. 1995). The island is home to an estimated $30,000-50,000$ tuatara and such a high density of tuatara (in some locations over 2,000 individuals per hectare; Moore et al. 2007, 2009b) makes the Stephens Island population an ideal source of founders for translocations. Cape Sanctuary, Young Nicks Head Sanctuary and Maungatautari are all fenced mainland sanctuaries located in New Zealand's North Island. Whangaokeno/East Island is the only unfenced site to which tuatara were translocated, but its location $\sim 2 \mathrm{~km}$ offshore places it beyond the swimming distance of introduced mammalian pests (King 1990; Nelson et al. 2002b).

Forty adults and 20 head-started juvenile tuatara were released into two separate 2,000 $\mathrm{m}^{2}$ enclosures within a separately fenced, 1.5 ha site at Cape Sanctuary. Young Nicks Head Sanctuary received 44 adults (released into the fenced 35 ha sanctuary) and 20 head-started juveniles, which were released into a smaller $5 \times 10$ m enclosure also within the fence. Head-started juveniles were hatched from wild-sourced eggs and raised in captivity until release. Whangaokeno also received 44 adults, which were released at three locations around the island whilst 50 adults were translocated to Maungatautari, with 30 released into a 35 ha enclosure on the mountain and 20 released into a $900 \mathrm{~m}^{2}$ enclosure in a separately fenced area known as the Tautari Wetland. The tuatara population at ZEALANDIA Ecosanctuary in Karori, Wellington, was also monitored for comparison purposes throughout this study. This sanctuary is located at the same latitude as Stephens Island and experiences a similar climate, enabling a comparison of the effect of translocation, rather than different environmental temperatures, though it was not possible to control for time since release. This sanctuary is surrounded by an $8.6 \mathrm{~km}$ pestproof fence and is home to 200 adult tuatara, which were translocated between 2005 and 2007 from Stephens Island (See Chapter Two section 2.2.1 for further detail on the source site and translocation sites). 


\subsubsection{Sample collection}

Tuatara were tested for intestinal carriage of Salmonella spp. and Campylobacter spp. at all four North Island sites, as well as Stephens Island and ZEALANDIA. Samples were collected opportunistically during four post-release survey seasons between November 2013 and May 2015 on adult and juvenile founders. Due to access limitations, not all sites could be visited in every season (Table 3.1). Searches for tuatara were conducted at night using head torches with white light. Cloacal swabs (Amies charcoal 114C and 116C; Copan, Brescia, Italy) were taken for Salmonella spp. and Campylobacter spp. screening from as many animals as possible in November 2013, with a maximum of 10 swabs collected per site on subsequent visits (Table 3.1). Swabs were not taken from the Maungatautari mountain enclosure population after November 2013. Tuatara had multiple measurements taken as part of a larger survey of the translocated populations (Chapter Two), including: snout-vent length (SVL), vent-tail length (VTL), tail regeneration length (if present) and mass. GPS location, sex, passive integrated transponder (PIT) identification number and toe-clip/beading identification (if present) were also recorded. Animals were visually checked for signs of injury or infection. After sampling, all animals were marked dorsally, at the base of the skull, with a black xylene-free marker pen to enable identification of already-captured animals, preventing unnecessary recapture during the survey. Cloacal swabs were taken from head-started juvenile founders at Young Nicks Head Sanctuary in spring 2013 in lieu of adult tuatara, which I was unable to access at that time. Cape Sanctuary juveniles did not have cloacal swabs taken as part of this study. Postcollection, swabs were labelled with an individual's PIT identification number, location and date, wrapped in bubble wrap and refrigerated or packed into a screw top plastic container with an ice pack to keep the samples cool. Samples were then transported to the Hopkirk Molecular Epidemiology Laboratory, Palmerston North, New Zealand, by courier or by hand. All samples arrived at the laboratory within 2-5 days of collection. Due to the opportunistic nature of tuatara captures, some individuals were sampled only once during this study whilst others had repeat samples taken across multiple seasons. One soil sample was also taken per site per season. Each soil sample consisted of a mix of 10 teaspoon-sized samples randomly collected from nearby to burrow entrances and accessible ground throughout the site. 
Table 3.1 Information on sites surveyed, tuatara population size, population density, number of tuatara captured and number/proportion of tuatara sampled in each season and overall. Adult tuatara were sampled exclusively at all sites other than Young Nicks Head, where both adults and juveniles were sampled. Seasonal sampling was classified as follows: spring: November-December; summer: February; autumn: March-May. Surveys were not conducted over June-August (winter) or December-January (early-mid summer). Percentage estimates of Stephens Island tuatara sampled vary with total estimated population sizes of 30,000 and 50,000 (see section 3.2.5 for details). Population densities displayed for Maungatautari reflect densities in the two enclosures at this site: Tautari Wetland $=222$, main mountain $=0.86$.

\begin{tabular}{|c|c|c|c|c|c|c|c|}
\hline Site & Pop. size & $\begin{array}{l}\text { Pop. density } \\
\text { (no. per ha) }\end{array}$ & Survey season & $\begin{array}{c}\text { No. } \\
\text { captures }\end{array}$ & $\begin{array}{c}\text { No. } \\
\text { swabs }\end{array}$ & $\begin{array}{l}\text { \% pop. } \\
\text { sampled }\end{array}$ & $\begin{array}{c}\text { Total \% pop. } \\
\text { sampled }\end{array}$ \\
\hline \multirow{4}{*}{ Cape Sanctuary } & \multirow{4}{*}{40} & \multirow{4}{*}{200} & Spring '13 & 19 & 19 & 48 & \multirow{4}{*}{78} \\
\hline & & & Autumn '14 & 21 & 10 & 25 & \\
\hline & & & Spring '14 & 23 & 10 & 25 & \\
\hline & & & Autumn '15 & 23 & 10 & 25 & \\
\hline \multirow{4}{*}{ Maungatautari } & \multirow{4}{*}{50} & \multirow{4}{*}{$\begin{array}{c}222 \\
\& 0.86\end{array}$} & Spring '13 & 17 & 17 & 34 & \multirow{4}{*}{44} \\
\hline & & & Autumn '14 & 14 & 10 & 20 & \\
\hline & & & Spring '14 & 18 & 10 & 20 & \\
\hline & & & Autumn '15 & 23 & 10 & 20 & \\
\hline \multirow{2}{*}{ Whangaokeno } & \multirow{2}{*}{44} & \multirow{2}{*}{3.39} & Summer '14 & 14 & 7 & 16 & \multirow{2}{*}{33} \\
\hline & & & Summer '15 & 8 & 8 & 18 & \\
\hline \multicolumn{8}{|l|}{ Young Nicks Head } \\
\hline Adults & 42 & 1.2 & Summer '15 & 7 & 7 & 17 & 17 \\
\hline Juveniles & 20 & 4,000 & Spring '13 & 13 & 13 & 65 & 65 \\
\hline \multirow{4}{*}{ ZEALANDIA } & \multirow{4}{*}{200} & \multirow{4}{*}{0.89} & Spring '13 & 23 & 16 & 8 & \multirow{4}{*}{16} \\
\hline & & & Autumn '14 & 24 & 10 & 5 & \\
\hline & & & Spring '14 & 19 & 10 & 5 & \\
\hline & & & Autumn '15 & 24 & 10 & 5 & \\
\hline \multirow{2}{*}{ Stephens Island } & \multirow{2}{*}{$30-50,000$} & \multirow{2}{*}{$200-333$} & Autumn '14 & 35 & 10 & $0.02-0.03$ & \multirow{2}{*}{$0.04-0.07$} \\
\hline & & & Autumn '15 & 43 & 10 & $0.02-0.03$ & \\
\hline
\end{tabular}




\subsubsection{Sample analysis: Campylobacter spp.}

I performed DNA extraction on cloacal swabs using the boiled preparation method. Angie Reynolds, of the mEpiLab at Massey University, Palmerston North, also performed DNA extractions from some samples in the spring '13 field season. Swab tips were cut into an Eppendorf Tube ${ }^{\circledR}$ (Thermo Fisher Scientific, Auckland, New Zealand) containing a suspension of $2 \%$ Chelex 100 resin (BioRad, Auckland, New Zealand) in sterile MilliQ water (Merck, Palmerston North, New Zealand), vortexed to mix and boiled for 10 minutes in a heating block at $100^{\circ} \mathrm{C}$. Soil samples were subjected to the same method, with cotton swabs used to collect a sample of soil for transfer to the Eppendorf Tube ${ }^{\circledR}$. Tubes were then removed and allowed to cool to room temperature before being centrifuged at $13,000 \mathrm{rpm}$ for three minutes. The supernatant was then extracted and stored at $-20^{\circ} \mathrm{C}$. Polymerase chain reaction (PCR) targeting the $16 \mathrm{~S}$ rRNA gene was performed on all samples using methods described in Linton et al. (1996) to provide genus level identification of Campylobacter spp. DNA extraction followed by PCR has been found to be a more sensitive diagnostic test than culture and PCR for Campylobacter spp. (Grange et al. 2015). The Linton et al. (1996) Campylobacter genus PCR has been found to have a sensitivity of $96 \%$ and specificity of $100 \%$ (On et al. 2013). Each PCR reaction was run with a preparation of Campylobacter jejuni as a positive control and sterile MilliQ water as a negative control. Gel electrophoresis was used to confirm successful amplification of PCR products and presence of Campylobacter spp.. Samples were run on a $1 \%$ gel agarose in Tris-borate-EDTA (TBE) buffer, which was then stained with ethidium bromide and exposed to UV light to make the products visible. An animal was classed as potentially positive for Campylobacter spp. if PCR and gel electrophoresis indicated a Campylobacter spp. positive sample.

As the PCR primers used (described in Linton et al. (1996)) can be cross-reactive with bacteria of the closely related Helicobacter and Arcobacter genera (A. Midwinter, pers. comm.) a subset of potentially Campylobacter-positive samples was sent for gene sequencing at Massey Genome Services, Palmerston North, New Zealand, for Campylobacter spp. confirmation and species-level identification. The first positive sample obtained from each animal that tested positive at least once during the study, irrespective of season, was added to the subset. In total, 111 samples were selected for 
sequencing. Prior to sequencing, PCR products underwent ethanol precipitation with ice cold $70 \% \mathrm{EtOH}$ and were rehydrated with sterile MilliQ water. Products were then nanodropped to obtain concentration, and, if necessary, diluted to $\sim 20 \mathrm{ng} / \mu \mathrm{l}$ for sequencing.

Two hundred and twenty-two $A B I$ sequence files resulted from the sequencing of 111 samples with both forward and reverse sequencing orientations. Sequence processing was considered as a pair of sequences from each sample. The $A B I$ files were loaded into Geneious (v. 7.1.8; http://www.geneious.com, Kearse et al. 2012), and the two sequences from each sample were placed into three bins based on their overall sequence quality as determined by Geneious; "good" (both sequences had a high quality (HQ) percentage of over 75\%), "maybe" (at least one sequence had a HQ percentage of over 40\%) and "bad" (all other sequences). The sequences in each of the three bins were then assembled with the Geneious assembler to generate an assembly. Consensus sequences were defined from the assemblies at a threshold of $75 \%$. The consensus sequences from each bin were then compared to each other to see how many unique consensus sequences resulted from the three bins. Sequences were then analysed using the MegaBLAST algorithm in BLAST ${ }^{\circledR}$ (Altschul et al. 1990). The MegaBLAST algorithm is used to search for highly similar nucleotide sequences in the National Centre for Biotechnology Information (NCBI) nucleotide databases and identify the most closely related organisms. Part of the MegaBLAST output includes an identity value, which informs the extent to which the sample nucleotide sequence aligns with similar sequences in the database, expressed as a percentage. An identity value of $98 \%$ and above is generally considered acceptable in confirming the identity of a sequence.

\subsubsection{Sample analysis: Salmonella spp.}

Selective culturing for Salmonella spp. followed a protocol adapted from Grange et al. (2015). Each sample was divided between two aerobic incubation treatments of $37^{\circ} \mathrm{C}$ and $28^{\circ} \mathrm{C}$. Transport media from each cloacal swab or $20 \mathrm{~g}$ of each soil sample were transferred into individual $20 \mathrm{ml}$ vials of buffered peptone water (BPW; BioRad, Auckland, New Zealand), vortexed to mix, then divided into two separate $10 \mathrm{ml}$ samples and incubated for 24 hours, one at $37^{\circ} \mathrm{C}$ and one at $28^{\circ} \mathrm{C}$. Following pre-enrichment, $200 \mu \mathrm{l}$ of 
BPW was inoculated into two selective enrichment broths, $100 \mu \mathrm{l}$ into $10 \mathrm{ml}$ of tetrathionate (TET) broth (Fort Richards, Auckland, NZ) enriched with iodine-iodide solution $(0.25 \mathrm{~g} \mathrm{Kl}, 0.3 \mathrm{~g} \mathrm{l} / \mathrm{ml} \mathrm{H} 2 \mathrm{O})$ and $100 \mu \mathrm{l}$ into $10 \mathrm{ml}$ of Rappaport-Vassiliadis Salmonella (RVS) broth, which were incubated for $24 \pm 2$ hours at their respective incubation temperatures. RVS and TET solutions were then streaked on to Xylose Lysine Deoxycholate (XLD) agar plates (Fort Richards, Auckland, NZ) and incubated for 18-24 hours at their respective temperatures. Two to four different suspect colonies (e.g. black, red with black centres, mustard-coloured colonies) were then selected from each XLD plate and subcultured on to MacConkey agar plates (Fort Richards, Auckland, NZ) and incubated for 18-24 hours at their respective temperatures. Any cultures on MacConkey agar that appeared grey in colouration (i.e. colonies that did not ferment lactose) were subcultured on to Triple Sugar Iron (TSI) and Lysine Iron Agar (LIA) slopes (Fort Richards, Auckland, NZ) and incubated for 18-20 hours at their respective temperatures. Any TSI agar slopes that presented as entirely yellow or LIA agar slopes with a yellow base were discarded as negative while all positive cultures underwent an oxidase test on Mast Oxidase Strips (Fort Richards, Auckland, NZ). Oxidase negative isolates were then tested for polyO and polyH antisera (Thermo Fisher Scientific, Auckland, New Zealand) reactivity and any positive isolates subcultured on to Columbia Horse Blood Agar (Fort Richards, Auckland, NZ) and incubated at $37^{\circ} \mathrm{C}$ for 24 hours. The identification of Salmonella spp. was then done by testing isolates grown on Columbia Horse Blood Agar using RapID inoculation fluid and RapID ONE panels (Thermo Fisher Scientific, Auckland, New Zealand) as detailed in the manufacturer's instructions. Any isolates identified by the RapID ONE panels as Salmonella spp. were cultured on to Dorset Egg agar slopes (Fort Richards, Auckland, NZ) and incubated at $37^{\circ} \mathrm{C}$ for 24 hours before being sent for serotyping at the Institute of Environmental Science and Research (ESR) laboratories in Wallaceville, New Zealand. As multiple suspect colonies could be selected from an individual sample, it was possible to obtain several positive isolates from a single swab/soil sample. Based on previous studies, this selective culturing technique is considered to have a sensitivity of $50 \%$ and specificity of $98 \%$ (Bager \& Petersen 1991). 


\subsubsection{Statistical analysis}

Analyses were performed in R v. 3.2.2 (R Core Team 2015). The EpiR package was used to assess Campylobacter spp. and Salmonella spp. apparent and true prevalence in each study population (Stevenson 2014). Apparent prevalence refers to the proportion of the sampled population found to be positive for the organism in question (i.e. Salmonella spp. or Campylobacter spp.) while true prevalence is an estimate of the number of animals in the population that could be positive. The true prevalence estimate is calculated using the apparent prevalence and the sensitivity and specificity of the diagnostic procedure used (e.g. PCR, selective culturing). To account for the potential for false negatives to influence apparent prevalence results within each survey, Fisher's exact tests were used to assess the influence of the categorical independent variables site and sex on overall Campylobacter spp. and Salmonella spp. apparent prevalence, calculated as the proportion of sampled individuals that tested positive for Campylobacter spp. or Salmonella spp. at least once during this study. I also investigated the association between population density and apparent prevalence across all surveys using a generalised linear mixed effects model (GLMM) with a binomial distribution (for presence/absence data) and individual ID as a random factor. Population density was calculated as the number of individuals in the population divided by the area of the site. However, while tuatara population density on Stephens Island has been found to vary with habitat type (Moore 2008; Moore et al. 2009b), as tuatara were active and moving during nocturnal surveys I was not able to determine the home location/habitat type of adult tuatara captured, therefore I calculated approximate population densities for Stephens Island as the estimated population size (30,000-50,000 animals) divided by the islands area (150 ha). This resulted in a population density estimate lower than has been observed in some habitats, but within the range of population densities previously estimated across the island (Cree 2014). 


\subsection{Results}

\subsubsection{Campylobacter: Apparent and true prevalence}

A total of 197 cloacal swabs and 19 soil samples were collected from 126 individual adult tuatara and six study sites between spring 2013 and autumn 2015. Apparent prevalence was calculated as the proportion of animals sampled per site that were identified as positive for Campylobacter spp. by Campylobacter genus PCR at least once during this study. A total of 113 adult tuatara were found to be positive for Campylobacter spp. by genus PCR, giving an overall apparent prevalence of $90 \%$ (95\% Cl: $83 \%-94 \%)$ and estimated true prevalence of $93 \%(95 \% \mathrm{Cl}: 86 \%-98 \%)$ for the sampled population. None of the head-started juvenile tuatara at Young Nicks Head Sanctuary were found to be positive for Campylobacter spp.. Due to differences in age, population history and postrelease exposure (i.e. juveniles were hatched from wild-collected eggs, raised in captivity prior to release, and then housed separately to the adult population and most other local fauna), the opportunities for exposure to these bacteria differ to the wild-caught adults and the juveniles were therefore not included in analyses of prevalence among adult populations. Apparent prevalence per site ranged between $84 \%$ and $100 \%$ of adult tuatara sampled (Figure 3.1) and true prevalence, the proportion of the total tuatara population that could be positive for intestinal carriage of Campylobacter spp., was estimated to range between $88 \%$ and $100 \%$ per site (Figure 3.1). I assessed the influence of site and an individual's sex on overall apparent prevalence of Campylobacter spp. in adults and found no significant relationship with these variables $(p>0.05)$. I also found no relationship between population density and apparent prevalence (Model $\chi 2=0.276, p=0.600$; Density: estimate $=43.192 \pm 69.137, z=0.625, p=0.532)$.

\subsubsection{Campylobacter species confirmation and characterisation}

Of the 113 tuatara that tested positive for Campylobacter spp., samples from 111 were sent for gene sequencing (two samples were weakly positive and eliminated from the sequencing subset). Campylobacter characterisation was then performed by Dr. Patrick Biggs at the mEpiLab, Massey University, Palmerston North. Of the 111 sequences, 42 ended up in the "good" bin, 44 in the "maybe" bin, and the remaining 25 in the "bad" bin. 
From each of the three bins, two consensus sequences resulted, and on comparison of all the sequences, only two separate sequences resulted. Therefore, across all 111 samples, irrespective of sequence quality as determined by Geneious, only two 16S rRNA consensus sequences resulted. Sequence one was closest to Campylobacter fetus subsp. testudinum, but was only $95 \%$ identical, so could be a member of the Campylobacter genus, and potentially a new species. Sequence two was most closely matched to Arcobacter butzleri ED-1, but was only 94\% identical; therefore, this sequence may be a member of the Arcobacter genus, and, again, could be a new species within the genus.

\subsubsection{Salmonella species serovars, and apparent and true prevalence}

Of the 197 cloacal swabs and 19 soil samples collected I found three isolates from one cloacal swab and three isolates from one soil sample to be positive for Salmonella enterica serovar Saintpaul. The cloacal swab that tested positive for S. Saintpaul had been collected during the first survey season, spring 2013, from an adult male tuatara that appeared clinically normal. This individual male was re-sampled in autumn 2014 and spring 2014, and the Cape Sanctuary soil re-sampled in all survey seasons until the end of the study, but no Salmonella spp. were isolated from any subsequent swabs or soil samples. This singular positive swab suggests a low estimated apparent prevalence of $S$. saintpaul of $3.23 \%(95 \% \mathrm{Cl}: 0.16 \%-16.69 \%)$ and an estimated true prevalence of $2.55 \%$ (95\% Cl: 0\%-30.61\%) in the Cape Sanctuary tuatara population (Figure 3.2). No Salmonella spp. were isolated from any other cloacal swabs or soil samples collected during this study, leading to apparent and true prevalence estimates of $0 \%$ (Figure 3.2). The small number of positive tuatara samples $(n=1)$ means that I was unable to assess the statistical significance of predictor variables such as site, season and sex on prevalence. 


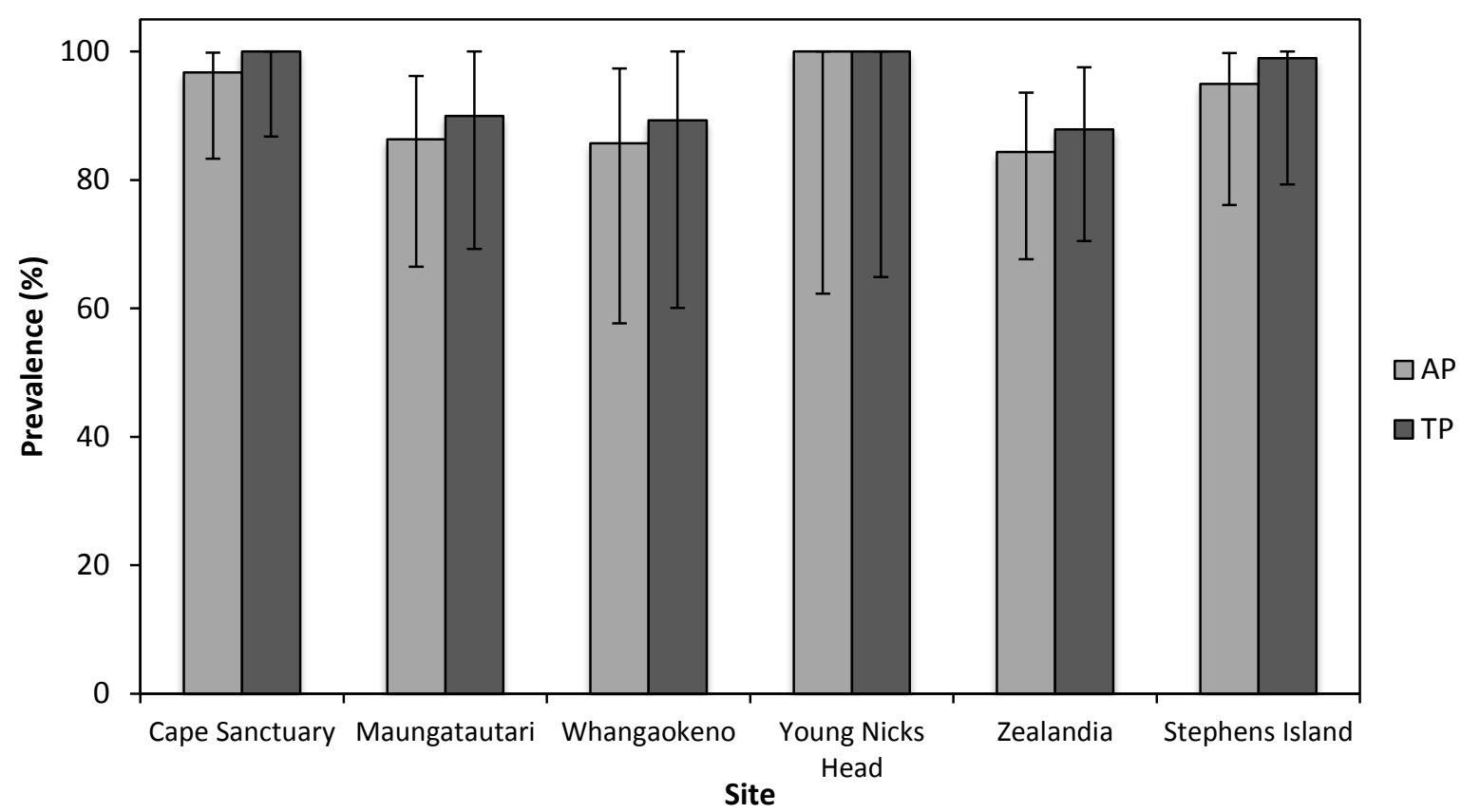

Figure 3.1 Bar plots of estimated apparent (AP) and true (TP) Campylobacter spp. prevalence in adult tuatara per site (as determined using cloacal swab samples). Apparent prevalence plot shows proportion of total sampled individuals identified as Campylobacter spp. positive at least once during the study. Error bars show $95 \%$ confidence limits. There was no significant effect of site on Campylobacter spp. apparent prevalence ( $p>0.05)$.

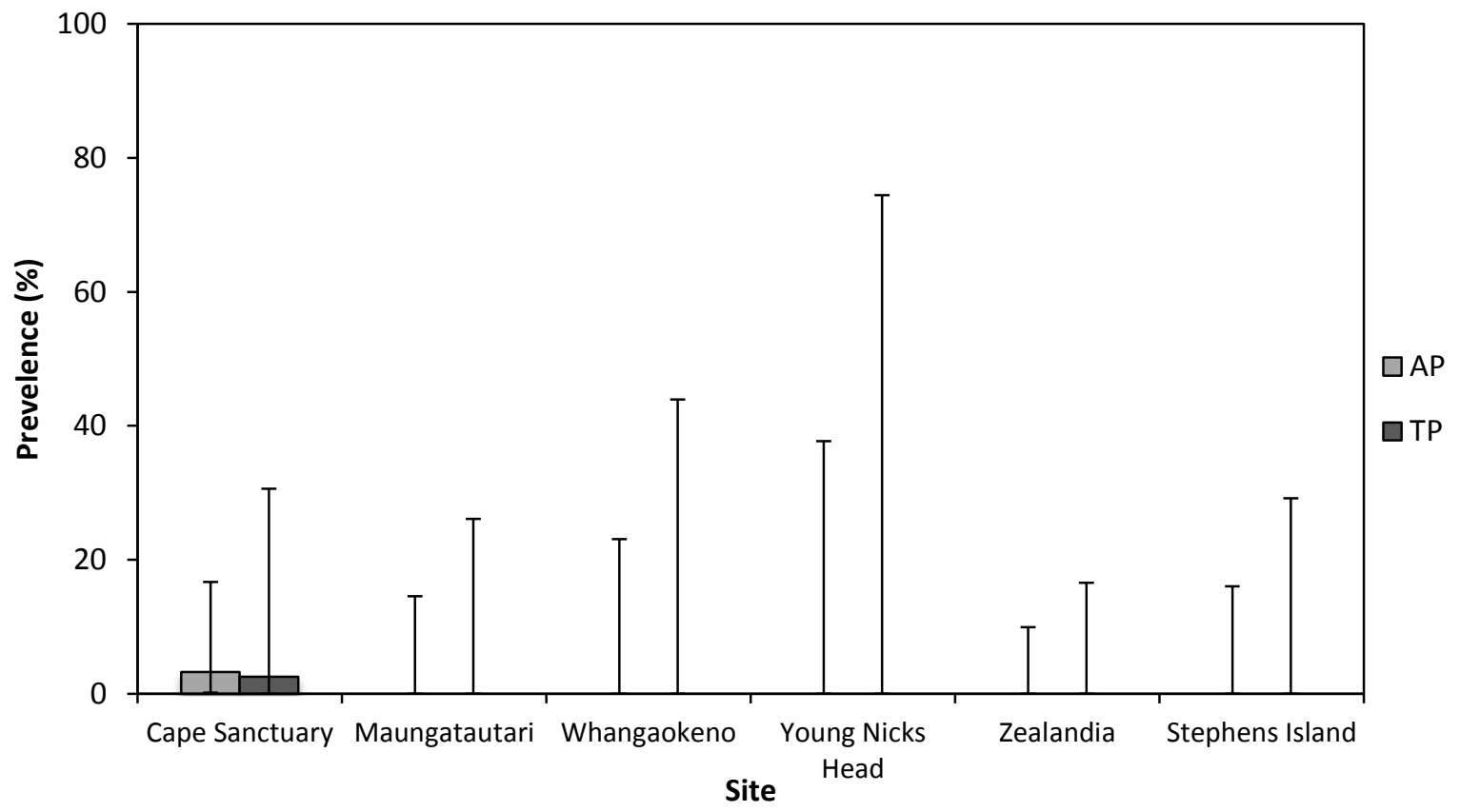

Figure 3.2 Bar plots of estimated apparent (AP) and true (TP) Salmonella spp. prevalence in adult tuatara per site (as determined using cloacal swab samples). Apparent prevalence plots (shown on the left) show proportion of total sampled individuals identified as Salmonella spp. positive at least once during the study. True prevalence values are shown on the right for each site. Error bars show 95\% confidence limits, which vary according to sample size and estimated population size. 


\subsection{Discussion}

Assessing bacterial prevalence in translocated populations is essential as results can highlight disease risks, contribute to the understanding of an organism's bacterial community, and in this case, inform predictions of how bacterial communities may be affected by translocations and rising temperatures predicted under anthropogenic climate change. Globally, salmonellosis has caused the deaths of wild birds, reptiles and mammals and S. Saintpaul has been associated with outbreaks in wildlife as well as human salmonellosis (De Hamel \& McInnes 1971; Baggesen et al. 1996; Bornemann et al. 2002; Iveson et al. 2009; Munnoch et al. 2009; Parsons et al. 2010; Taylor et al. 2010). Within New Zealand, Salmonella enterica subspecies houtenae has been implicated as the cause of death in an adult female tuatara (van Andel et al. 2014). Similarly, native lizards and soil have been identified as reservoirs for $S$. Saintpaul and this serovar has been implicated in the deaths of native birds and sheep (McInnes 1971; Middleton et al. 2010, 2014; Baling et al. 2013a; van Andel et al. 2014). However, prior to this study there had been no reports in the literature of isolation of a Salmonella sp. from a live tuatara, which had led to hypotheses that this species may be innately resistant to gastrointestinal colonisation by Salmonella spp., that intermittent shedding was resulting in false negatives, or that tuatara were not exposed to the bacteria, which is unlikely given the prevalence of Salmonella spp. in the environment (Gartrell et al. 2007; Middleton et al. 2014, 2015).

This study marks the first time that a Salmonella sp. has been isolated from a live tuatara and suggests that $S$. Saintpaul is able to colonise the tuatara gastrointestinal tract at least temporarily. I was able to detect $S$. Saintpaul in a cloacal swab from an adult male that had been translocated to Cape Sanctuary and the same serovar was also detected in the soil of the Cape Sanctuary enclosure. Both the male and soil were found to be positive for S. Saintpaul in spring 2013 and no other sampling seasons. Considering that this is the first positive detection of a Salmonella sp. in a live tuatara, the potential for this to represent a false-positive result must be considered. Potential contamination routes were considered in the methodology and samples were carefully collected by inserting swabs directly into the cloaca to avoid swabbing the surrounding environmentally contaminated skin. Similarly, all lab analyses were performed using aseptic techniques and there were no opportunities for cross-contamination between cloacal and soil samples. The use of $S$. 
typhimurium as a positive control also eliminates the potential for S. Saintpaul detection to be a result of contamination with control colonies grown in the lab. This small number of positive samples means I was unable to perform any meaningful statistical analyses on the effect of translocation, site, season or tuatara characteristics on prevalence and so cannot infer that translocation or climate will affect Salmonella spp. prevalence in tuatara, although previous work on New Zealand lizards has found no influence of translocation on Salmonella spp. prevalence (Middleton et al. 2010; Baling et al. 2013a). However, this finding illustrates that $S$. Saintpaul true prevalence estimates $(0 \%-2.55 \%)$ for tuatara populations are comparable to Salmonella spp. true prevalence data documented for other New Zealand reptiles (2.3-8.8\%) and lower than estimates for overseas reptile populations (Hoff \& White 1977; Otokunefor et al. 2003; Chambers \& Hulse 2006; Middleton et al. 2010; Franco et al. 2011; Scheelings et al. 2011; Bull et al. 2012; Baling et al. 2013a; Marin et al. 2013).

That the male appeared clinically normal each time he was examined suggests that S. Saintpaul may have had a non-pathogenic association and was carried asymptomatically by this tuatara. Alternatively, the Salmonella serovar was only isolated on a single occasion from this tuatara and may therefore have represented the transient passage of the Salmonella bacteria rather than a true colonisation event. However, Salmonella spp. are opportunistic bacteria that can become pathogenic if the host is immunocompromised, so there remains a risk that the bacteria could cause disease in this individual (Carey 1993). As Cape Sanctuary has a warm climate and experiences periods of drought in summer months it is possible that sub lethal environmental stressors could impair the immune system and enable systemic infection (Carey 1993). The closely related S. enterica subspecies houtenae was implicated as the cause of death in an adult female tuatara found to have been in very poor body condition on Tiritiri Matangi Island, illustrating the mortality risk in tuatara (van Andel et al. 2014). As. Saintpaul was isolated from only one study site this also suggests that there may be transmission routes or reservoirs present at Cape Sanctuary that are not present at others. There are multiple potential routes by which an individual could become infected with a Salmonella sp. and which could explain its carriage in the Cape Sanctuary male. The identification of S. Saintpaul in the soil supports previous research that suggests that contamination of the environment is a potential reservoir for infection, with Salmonella spp. able to remain 
viable outside of a host for, in some instances, over a year (Thomason et al. 1977; Chao et al. 1987; Winfield \& Groisman 2003). However, in the absence of whole genome sequencing to determine the relatedness of the soil and tuatara isolates, we cannot confirm transmission between the two. There is no natural water source in the tuatara enclosure at Cape Sanctuary so water is provided in artificial dishes that are refilled as necessary using roof-collected rainwater. Several species of wild birds and lizards are known to carry Salmonella and previous work has isolated Salmonella enterica serovar Typhimurium from roof-collected rainwater in New Zealand, citing bird faeces as the possible source of contamination (Koplan et al. 1978; Simmons et al. 2001; Alley et al. 2002; Franklin et al. 2009; Middleton et al. 2010, 2014). Similarly, local birds have been observed bathing in the enclosures' water dishes and it is probable that resident skink populations and visiting seabirds make use of or come into contact with them, offering multiple routes by which the water could become contaminated. As tuatara prey on small birds and lizards it is also feasible that direct contact through predation could facilitate transmission. If we consider the singular isolation of a Salmonella sp. from this tuatara as indicative of a transient gut passage, it is possible that the positive cloacal swab could have detected $S$. Saintpaul in ingested soil or an infected prey item (e.g. skink) passing through the gastrointestinal tract. Similar cases have occurred in which the parasitic protozoa Monocystis have been identified in kiwi (Apteryx) faeces, which were in fact parasites of the kiwi's earthworm (Lumbricidae) prey (Jakob-Hoff 1998).

Campylobacter spp. are considered commensal bacteria in many taxa, but prior to this study little was known about prevalence of Campylobacter spp. in tuatara. While they have not been reported to cause disease in reptiles, Campylobacter spp. have been detected in wildlife, water and livestock in New Zealand and are prominent zoonotic pathogens associated with gastrointestinal diseases in humans (Eberhart-Phillips et al. 1997; French et al. 2009; Mullner et al. 2009; Man 2011; Gilbert et al. 2014). This study found high Campylobacter spp. prevalence in all translocated populations studied as well as the control population at ZEALANDIA and the source population on Stephens Island. I found no relationship between site or sex and prevalence and the high true prevalence estimates (88\%-100\%) indicate that Campylobacter spp. could be considered common commensals with a non-pathogenic association in tuatara, similar to captive and wild 
reptiles around the world (Marin et al. 2013; Wang et al. 2013; Giacomelli \& Piccirillo 2014; Gilbert et al. 2014).

Given that Campylobacter spp. were not detected in any of the head-started juvenile tuatara at Young Nicks Head Sanctuary, and that prevalence did not differ significantly between the Stephens Island population and any of the translocated populations, suggests that initial infection occurred while animals were resident on Stephens Island and has persisted post-translocation, indicating that there has been no impact of translocation itself on bacterial prevalence. Similar prevalence values across comparatively warmer and cooler translocation sites also suggest that warmer climates have had no measurable impact on Campylobacter spp. prevalence and imply that warming air temperatures predicted under anthropogenic climate change may not affect carriage of Campylobacter spp., though this conclusion cannot be generalised to other bacterial genera.

I found that four individuals from the Cape Sanctuary, Maungatautari and Whangaokeno populations tested negative when first captured, but were found positive for Campylobacter spp. in subsequent seasons, providing evidence that transmission may have occurred among individuals or via an alternative reservoir while at the translocation site, although intermittent shedding of bacteria could also explain negative results (Gartrell et al. 2007). Similarly, two individuals at ZEALANDIA returned positive-negativepositive results across three survey seasons, which could be demonstrative of re-infection or intermittent shedding. These findings also indicate that further work is required to determine whether Campylobacter spp. prevalence in the Young Nicks Head juvenile tuatara has changed since the spring 2013 sampling. As the juveniles were raised in relative isolation (captivity) and were contained in a separate enclosure to the adults following release, this may have limited exposure opportunities (although the enclosure's wire mesh structure feasibly allowed for transmission via small animals or bird faeces) resulting in zero per cent prevalence. In 2016, ten of these juveniles were released into the wider sanctuary, offering opportunities for direct contact with adult tuatara and other fauna (S. Sawyer, pers. comm.).

We were unable to identify either of the two separate sequences that resulted from our samples to the Campylobacter genus or species level after gene sequencing so cannot confirm which Campylobacter species are present in tuatara or assess whether 
there are site-dependent differences in Campylobacter species prevalence. It is possible that we did not find significant sequence alignments in the NCBI databases because the bacterial species isolated have not been previously identified and documented, meaning that the tuatara sampled in this study could have intestinal carriage of two novel bacterial species. However, it is important to acknowledge limitations of the PCR procedure when considering implications of the gene sequencing outcomes. The Campylobacter genus PCR used in this study is considered to be $96 \%$ sensitive and $100 \%$ specific to Campylobacter spp. bacteria; however, the potential for cross-reactivity with closely related Helicobacter spp. and Arcobacter spp. has been noted (Inglis \& Kalischuk 2003; On et al. 2013, A. Midwinter pers. comms.). Consequently, it is possible that some of the samples identified as Campylobacter spp. by genus PCR were in fact of the genera Helicobacter or Arcobacter, therefore tuatara could alternatively have intestinal carriage of novel Helicobacter or Arcobacter species. In the absence of bacterial genus/species identification, I must clarify that the apparent and true Campylobacter spp. prevalence reported could consequently be lower than my calculations estimate, and suggest that further work to culture these bacteria is necessary for identification, though attempts to do so have so far proved unsuccessful (A. Midwinter, pers. comm.).

Limitations of the sampling and experimental procedures must also be considered when interpreting these results. The failure to detect $S$. Saintpaul in the Cape Sanctuary male following the initial positive result is not confirmation that the male was not still a carrier of S. Saintpaul, only that he was not shedding at the time of sampling (Gartrell et al. 2007). The same can be said of other tuatara that tested negative for Salmonella spp. and of individuals that did not repeatedly test positive for Campylobacter spp.. Equally, the selective culturing techniques used are assumed to be $50 \%$ sensitive to Salmonella spp., which could also lead to false negatives and offer an alternative explanation for negative results in subsequent seasons (Bager \& Petersen 1991). However, it is important to note that this sensitivity estimate is based on a study isolating Salmonella spp. from domestic pig (Sus scrofa domesticus) faeces, so the relevance of these values to detection of Salmonella spp. using tuatara cloacal swabs may be questionable. Whilst the Campylobacter genus PCR used has a much higher sensitivity (96\%) there is still potential for positive samples to be classed as negative and therefore underestimate prevalence. Equally, the quality and quantity of DNA extracted from cloacal swabs can influence the 
detection of target bacteria (Inglis \& Kalischuk 2003). Monitoring limitations associated with habitat accessibility and detection of cryptic tuatara and restrictions to the number of cloacal swabs that could be processed each survey season also affected the proportion of each population that could be tested for bacterial carriage. The potential for false negatives and possibility that we failed to sample individuals that may have been shedding means that Salmonella spp. and Campylobacter spp. prevalence estimates may in reality be higher than those calculated in this study.

In summary, this study demonstrates that Campylobacter spp. are likely common commensal bacteria in tuatara and marks the first time that a Salmonella $\mathrm{sp}$. has been isolated from a live tuatara. However, the small sample size and possibility that the $S$. Saintpaul detected was a transient gut inhabitant prevents any conclusions regarding the effect of translocation or climate on Salmonella spp. prevalence in tuatara. While results suggest that Campylobacter spp. prevalence has been unaffected by the act of translocation or movement to warmer climates, the detection of a high prevalence of unidentified Campylobacter spp. indicates that identification of the species and strain variations within the Campylobacter genus may provide a better model for studying the effects of translocation and climate change on bacterial colonisation in tuatara. However, to achieve this I will need to employ more than presence/absence detection methods when monitoring changes in bacterial colonisation and incorporate molecular tools such as whole genome sequencing. 


\section{Chapter Four}

\section{Relationships between climate, population density and timing of translocation on parasites of tuatara (Sphenodon punctatus)}

\subsection{Introduction}

Parasites can negatively impact parameters ranging from growth and behaviour to fecundity and survival, and pose a substantial threat to the conservation of their hosts (Scott 1988; Brown et al. 1995; Moller \& Nielsen 2007; Zamora-Vilchis et al. 2012; Reisinger et al. 2015). At the same time, parasites can contribute to the maintenance of genetic diversity, regulate host population size, mediate competition between host species and influence community composition, and can be integral to host ecosystems (Hudson et al. 1992; Hudson \& Greenman 1998; Windsor 1998; O'Brien 2000; Harvell et al. 2002; Godfrey et al. 2008). Climate plays a significant role in the extent to which a parasite is able to infest hosts through effects on both organisms and their interaction. Exposure of hosts to stressful abiotic conditions (e.g. unfavourable temperatures, drought) or events (e.g. pollution, predation, translocation) as well as seasonal fluctuations in hormone levels can cause immunosuppression, and poor individual fitness can lead to a lower immune response, which can facilitate increased parasite prevalence and infection intensity (Esch et al. 1975; Oppliger et al. 1998; Klukowski \& Nelson 2001; Cichoń et al. 2002; Navarro et al. 2003; De Kloet et al. 2005; Ujvari \& Madsen 2006; Cox \& John-Alder 2007; Teixeira et al. 2007). Consequently, increased air temperatures, adverse environmental conditions, and ecological disturbances predicted under anthropogenic climate change could facilitate increases in parasite abundance, which could be substantially detrimental to host populations (Brooks \& Hoberg 2007).

While adverse biotic and abiotic factors can increase host vulnerability to parasitism, the exposure of parasites to different thermal climates can also facilitate changes in parasite prevalence. Endoparasites, which live inside of the host, occupy a comparatively stable thermal environment and are less susceptible to changing air temperatures, whereas ectoparasites, which live outside of the host, are directly affected and are more sensitive to climatic changes (Bush et al. 2001; Møller 2010). Consequently, 
environmental temperature is especially influential to ectoparasites of ectothermic hosts, whose physiological function is strongly affected by the immediate thermal environment and offers ectoparasites little protection against changing climatic conditions (Deutsch et al. 2008; Polley \& Thompson 2009). Ectoparasites are therefore likely to experience different thermal challenges and potential advantages to endoparasites under climate change, but responses will also be mediated by factors like host-specificity, host density/mobility and the physiological tolerances of the parasite (Martinez \& Merino 2011). Warmer temperatures can enhance biochemical activity, speed up parasite growth and development rates, prompt reproduction and advance life-cycle completion rates, whereas low temperatures can reduce parasite abundance through the inhibition of development and slowing of life cycles, processes which have been observed in species of tapeworm, parasitoid wasps, ticks and mites (Nealis et al. 1984; Perring et al. 1984; Oliver 1989; Chilton \& Bull 1991; Ogden et al. 2004; Godfrey et al. 2008; Lafferty 2009; MacNab \& Barber 2012; IPCC 2014). However, maintaining an increased temperaturedependent metabolic rate would also require higher resource consumption and could result in decreased parasite survivorship if energetic demands are not met, which is a particular risk for parasites with free-living developmental stages (King \& Monis 2007; Lafferty 2009). Equally, warmer temperatures could disturb seasonal reproductive cycles, disrupt host-parasite synchronies and, if rising temperatures exceed those optimal for parasite growth and transmission, climate change also has the potential to bring about a decrease in parasite prevalence, though it is possible that such temperatures would also be detrimental to the host (Polley \& Thompson 2009; Karvonen et al. 2010).

Changes in the distribution of endoparasite vectors associated with a warming climate could also result in changes in parasite distribution. As vector abundance and the transmission of parasites and pathogens can be enhanced by higher ambient temperatures, rising air temperatures are predicted to facilitate range expansions and shifts of vectors (i.e. mosquitoes, ticks, fleas), resulting in consequent expansions/shifts of the endoparasites and pathogens they transmit (Lindsay \& Birley 1996; Patz \& Reisen 2001; Cumming \& van Vuuren 2006; Zamora-Vilchis et al. 2012). Such expansions/shifts could increase endoparasite and pathogen prevalence in host populations, bringing with them the associated impacts on host reproduction, growth and survival and intensifying the risk of population extinctions (Zamora-Vilchis et al. 2012). Associations between 
warmer temperatures in lowland regions and increased vector and haemoparasite abundance has been observed in numerous bird species in Australia (Zamora-Vilchis et al. 2012). Similarly, an increase in the number of cases of tick-borne encephalitis in Europe have been partly attributed to climate-associated range expansion of the tick Ixodes ricinus, and climate has been frequently associated with plague outbreaks facilitated by flea vectors (Randolph 2004; Gray et al. 2009; Ari et al. 2011). However, complex interactions between aspects of host, vector and parasite/pathogen ecology, epidemiology and environmental factors (i.e. landscape heterogeneities and barriers to movement) can make it difficult to determine precise causes of changes in prevalence (Lindgren \& Jaenson 2006; Gray et al. 2009; Ari et al. 2011).

Anthropogenic climate change is a significant conservation concern and is expected to have substantial effects on biodiversity (Parmesan 2006; IPCC 2014). Conservation interventions like translocations have been employed to remove animals from unsuitable habitats and extend the range of vulnerable species, theoretically reducing the likelihood of extinction (Thomas 2011; IUCN/SSC 2013). To minimise the risk of translocation failure, it has been recommended that species are released into high quality habitat within their historical range and that the initial cause of species decline is addressed prior to translocation (Griffith et al. 1989; Dodd \& Seigel 1991; Wolf et al. 1996; Fischer \& Lindenmayer 2000; Germano \& Bishop 2008). However, certain risks can be difficult to mitigate. Although disease screening is often implemented to prevent the transmission of novel pathogens and parasites into naïve local populations, translocated animals are vulnerable to spillover from local reservoirs and are generally expected to acquire parasites and pathogens post-release (Leighton 2002; Gartrell et al. 2006, 2007; Germano \& Bishop 2008; Kock et al. 2010; Almberg et al. 2012). As increased disease prevalence has been linked to translocation failure and parasites themselves can be pathogenic or act as vectors for other pathogens, it is important that parasite status is monitored post-release so that potential threats to the translocated population can be quickly detected (Cunningham 1996; Daszak et al. 1999; de la Fuente et al. 2008). Equally, as many species of parasite are host-specific, they can be as vulnerable and important to biodiversity as their hosts, and there may be an ethical obligation to ensure that parasite assemblages are maintained post-translocation (Daszak et al. 2000). 
The tuatara (Sphenodon punctatus) is a long-lived ( 100y), cold-adapted, nocturnal New Zealand endemic reptile and the sole extant representative of the Order Rhynchocephalia (Gaze 2001; Besson \& Cree 2010; Cree 2014). Tuatara were once widespread throughout New Zealand, but the introduction of mammalian predators saw them restricted to predator-free offshore islands (Chapter One, Figure 1.1; Cree \& Butler 1993; Anderson 1996; Holdaway \& Worthy 1997). Conservation translocations have since served to extend their current range, but limited dispersal ability and a cold-adapted lifestyle place tuatara at particular risk from increasing air temperatures (Miller et al. 2012; Hitchmough et al. 2016; Cree 2014). However, this cold-adaptation also makes tuatara an ideal species for studying the early effects of climate warming on reptiles and their parasite communities.

Tuatara are host to and arachnid ectoparasite, the tuatara tick (Amblyomma sphenodonti), two species of trombiculid mite (Neotrombicula sphenodonti and Neotrombicula naultini) and an apicomplexan haemoparasite (Hepatozoon tuatarae), which may be transmitted by A. sphenodonti (Dumbleton 1943; Goff et al. 1987; Godfrey et al. 2010a). Coccidial oocysts, nematode ova, and motile protozoa have been documented in faeces of captive-reared tuatara and the nematode Hatterianema hollandei and the trematode Dolichosaccus (Lecithopyge) leiolopismae have also been documented in tuatara (McKenna 2003; Gartrell et al. 2006). Both H. tuatarae and A. sphenodonti are tuatara-specific parasites whilst mites (Neotrombicula spp.) are also found on other New Zealand reptiles (Godfrey et al. 2008, 2010a). Infestation with ticks and mites has been shown to be associated with reduce body condition, a measure of relative body bulk (Cree 2014), in tuatara; however no correlation between $H$. tuatarae and body condition has been found and there is low overall prevalence and infection intensity in the most studied population of tuatara (Godfrey et al. 2010a, 2011a).

Transmission of $A$. sphenodonti and Neotrombicula spp. to their hosts requires direct contact, so host and parasite must occupy the same environment for attachment to occur (Godfrey et al. 2011b). A. sphenodonti feed on a host during each of three developmental stages (larvae, nymph, adult), detaching once engorged to moult into the next stage or for females to lay eggs (Dumbleton 1943). Adult $A$. sphenodonti have been shown to survive for long periods off-host (males: up to 582 days; females: up to 405 days) when maintained in constant temperature and high humidity laboratory conditions $\left(18^{\circ} \mathrm{C}\right.$, 
90-95\%; Heath 2006). However, as maintaining body-water equilibrium is key to off-host survival, desiccation as well as eventual starvation pose significant risks during free-living periods if reattachment does not occur (Oliver 1989; Needham \& Teel 1991). As such, ticks are unlikely to travel far and $A$. sphenodonti is thought to employ a "sit-and-wait" strategy, residing in refuges frequented by tuatara (Kerr \& Bull 2006; Godfrey et al. 2010a, 2011b). Accordingly, laboratory microcosm and activity experiments have found that $A$. sphenodonti, like their hosts, are most active at night and show microhabitat preferences for moist, coarse, and shaded substrates, comparable to the inside of a tuatara burrow, thus reducing desiccation risk and maximising reattachment opportunities (Heath 2006; Kerr \& Bull 2006; Godfrey et al. 2011b). Conversely, mites only attach to tuatara in the larval stage, during which time they cluster in areas where they are least exposed to intense abiotic conditions or likely to be brushed off during movement (Arnold 1986; Godfrey et al. 2010a).

It is expected that local and translocated wildlife will come into contact with new parasites and pathogens following translocation and it is possible that translocationassociated stressors (e.g. capture, examination, transport, release into a new environment) could elevate glucocorticoid levels (Dickens et al. 2010). The prolonged elevation of these stress hormones may reduce host immunocompetence and increase the vulnerability of translocated animals to infection, though the relationship between elevated glucocorticoids and susceptibility to infection has not been investigated in tuatara (Teixeira et al. 2007; Godfrey et al. 2008). While the tuatara-specific nature of $A$. sphenodonti and $H$. tuatarae reduces the risk of transmission between tuatara and local reptiles at release sites, the same cannot be said of trombiculid mites, the larvae of which can parasitise a wide range of hosts from multiple taxa, with high abundance negatively associated with host reproduction, growth rate and survival (Klukowski \& Nelson 2001; Curtis \& Baird 2008; Lin et al. 2014). For instance, the mite Trombicula alfreddugesi has been observed on 52 avian, 39 reptilian, 32 mammalian and three amphibian species while $N$. sphenodonti and $N$. naultini have also been observed on skinks and geckos in New Zealand, though prevalence in lizard populations at the translocation sites monitored in this study has not been determined (Wharton \& Fuller 1952; Arnold 1986; Benton 1987; Godfrey et al. 2008, 2010a). This lack of host-specificity contributes to the risk of trombiculid mites as vectors of disease and the mites feeding action can cause direct 
damage to the host's skin, leaving the resulting wound vulnerable to secondary infection (Arnold 1986).

This study utilised several large-scale tuatara translocations to assess relationships between warmer climates and the act of translocation on parasite prevalence and load in tuatara. In October 2012, 176 adult tuatara were translocated from Takapourewa/Stephens Island in the Cook Strait to four sites around New Zealand's North Island: Maungatautari Ecological Reserve, Whangaokeno/East Island, Cape Sanctuary and Young Nicks Head Sanctuary (Chapter One, Figure 1.1). These translocations were designed to benefit conservation goals and all release sites are protected, pest-free sanctuaries within the species historical range (Worthy \& Holdaway 2002; Wood 2009; IUCN/SSC 2013). The comparatively warmer, drier climates of the translocation sites (with the exception of Maungatautari, which experiences comparatively higher humidity levels) are on average $2-4^{\circ} \mathrm{C}$ warmer than Stephens Island during summer months (December February), and provide surrogates for temporal climate change, enabling an assessment of how tuatara and parasite assemblages may be affected as the climate warms (Chapter Two, Figures 2.1 and 2.2). Though it is important to acknowledge that other biotic and abiotic factors will vary. Declines in tick prevalence's have been observed following translocation (McKenzie 2007; Moir et al. 2012) and it has been suggested that lower density translocated populations may be unable to sustain tick populations (Miller et al. 2007). Therefore, these translocations also enable an assessment of how populations of the endangered, host-specific tuatara tick, $A$. sphenodonti, might be impacted following host translocation (Buckley et al. 2012). I investigated the impact of translocation and movement to warmer climates on the prevalence of a range of ecto- and endo-parasites in tuatara. This information will be important for informing future translocations and conservation management strategies for tuatara, and has wider implications for understanding how cold-adapted reptiles and their parasitic assemblages may be impacted by rising air temperatures associated with anthropogenic climate change. 


\subsection{Methods}

\subsubsection{Founder and translocation sites}

Stephens Island is a pest-free, 150 ha offshore island nature reserve in the Marlborough Sounds and is a site of high conservation significance (East et al. 1995; DOC 2006). The high density of tuatara (over 2,000 per hectare in some forested areas; Moore et al. 2007, 2009b) on Stephens Island makes this population ideal for translocation. All of the translocated tuatara populations monitored in this study were sourced from Stephens Island.

Cape Sanctuary, Young Nicks Head Sanctuary and Maungatautari are all fenced mainland sanctuaries located in New Zealand's North Island. Whangaokeno/East Island is the only unfenced site to which tuatara were translocated, but its location $\sim 2 \mathrm{~km}$ offshore places it beyond the swimming distance of introduced mammalian pests (King 1990). Forty adults and 20 head-started juvenile tuatara were released into two separate 2,000 $\mathrm{m}^{2}$ enclosures within a separately fenced, 1.5 ha site at Cape Sanctuary. Young Nicks Head Sanctuary received 44 adults (released into the fenced 35 ha sanctuary) and 20 headstarted juveniles, which were released into a smaller $5 \times 10$ m enclosure inside the fence. Whangaokeno also received 44 adults, which were released at three locations around the island whilst 50 adults were translocated to Maungatautari, with 30 released into a 35 ha enclosure on the mountain and 20 released into a $900 \mathrm{~m}^{2}$ enclosure in a separately fenced area known as the Tautari Wetland.

The tuatara population at ZEALANDIA ${ }^{\mathrm{TM}}$, Wellington, was also monitored for comparison purposes throughout this study. This sanctuary is surrounded by an $8.6 \mathrm{~km}$ pest-proof fence and is home to 200 adult tuatara, which were translocated in 2005 and 2007 from Stephens Island. The preliminary translocation of 70 adult tuatara in December 2005 was monitored for one year following release (January-December 2006) by McKenzie (2007). These data have been used here for post-translocation comparison purposes (See Chapter Two section 2.2.1 for further detail on the source site and translocation sites). 


\subsubsection{Translocations}

Translocations from Stephens Island were performed in October 2012. Adult tuatara were captured at night and each animal had details of sex, parasite load (i.e. presence/absence of ticks and mites) and morphometric measurements taken. No parasites were removed from animals prior to or following translocation (see Chapter Two section 2.2.2 for further detail on the translocations). Tuatara were released into artificial burrows (e.g. buried corrugated plastic/terracotta pipes, bore holes) at translocation sites within 12 hours of departure from Stephens Island.

Head-started juvenile tuatara were translocated to Cape Sanctuary and Young Nick Head Sanctuary in March 2012. Head-started juveniles were hatched from wild-laid eggs collected from Stephens Island and incubated at Victoria University of Wellington in Wellington, New Zealand (DOC permit NM-18922-CAP). According to standard protocols followed in previous studies, juveniles were then raised at a captive-rearing facility in semi-natural enclosures until $\sim 5$ years of age (Nelson et al. 2002b). The juveniles were transported from the captive-rearing facility in Wellington to Cape Sanctuary and Young Nicks Head Sanctuary by car and aeroplane and released into artificial burrows in their respective enclosures within 24 hours.

\subsubsection{Opportunistic monitoring}

Tuatara populations were surveyed over four post-release sessions between November 2013 and May 2015 (Table 4.1). Due to access limitations, not all sites could be visited in every season. Searches were conducted at night using head torches with white light and any observed tuatara were caught by hand. Captured individuals had multiple measurements taken as part of a larger survey of the translocated populations (Chapter Two), including: snout-vent length (SVL), vent-tail length (VTL), tail regeneration length (if present) and mass. GPS location, sex and individual ID were also recorded. The total parasite load (i.e. number of individual ticks (of all life stages) and mites) for each tuatara was counted (Figure 4.1). If mites were tightly clustered (as they often were) numbers were estimated based on counts of mites within a defined area, which was then extrapolated by eye to estimate cluster size (Godfrey et al. 2008). The same individual (S. 
Price) always performed ectoparasite counts and estimates. Faecal samples were collected opportunistically after an animal defecated post-capture or if an animal was found beside a fresh sample. Animals were visually checked for signs of injury or infection. All animals were marked dorsally, at the base of the skull, with a black xylene-free marker pen to enable identification of those already captured and prevent unnecessary recapture during the survey. Juveniles at Young Nicks Head were monitored in spring 2013 and summer 2014 in lieu of the adult population, which was inaccessible at that time. As we were able to examine the adult population at Cape Sanctuary throughout this study, parasite prevalence in the juvenile population was not monitored.

Table 4.1 Information on sites surveyed, tuatara population size, number of tuatara captured in each survey and number of faecal samples obtained. Data are for adult tuatara unless juveniles were also surveyed (i.e. Young Nicks Head). Seasonal surveys were classified as follows: spring: November-December; summer: February; autumn: March-May. Surveys were not conducted over June-August (winter) or December-January (early-mid summer). Tuatara at Maungatautari were released into two separate enclosures. Twenty into a 0.09 ha wetland enclosure and 30 into a 35 ha forest enclosure. Percentage estimates of Stephens Island tuatara vary with total estimated population sizes of 30,000 and 50,000 (see Chapter 3, section 3.2.5 for details).

\begin{tabular}{|c|c|c|c|c|c|c|}
\hline Site & $\begin{array}{l}\text { Area } \\
\text { (ha) }\end{array}$ & $\begin{array}{l}\text { Pop. } \\
\text { size }\end{array}$ & $\begin{array}{c}\text { Pop. } \\
\text { density (no. } \\
\text { per ha) }\end{array}$ & $\begin{array}{l}\text { Season } \\
\text { surveyed }\end{array}$ & $\begin{array}{c}\text { No. } \\
\text { captures }\end{array}$ & $\begin{array}{c}\text { No. } \\
\text { faecal } \\
\text { samples }\end{array}$ \\
\hline \multirow{4}{*}{ Cape Sanctuary } & \multirow{4}{*}{0.2} & \multirow{4}{*}{40} & \multirow{4}{*}{200} & Spring ‘13 & 19 & 1 \\
\hline & & & & Autumn '14 & 21 & 0 \\
\hline & & & & Spring '14 & 23 & 1 \\
\hline & & & & Autumn '15 & 23 & 1 \\
\hline \multirow{4}{*}{ Maungatautari } & \multirow{4}{*}{$\begin{array}{l}0.09 \\
\& 35\end{array}$} & \multirow{4}{*}{50} & \multirow{4}{*}{$\begin{array}{r}222 \\
\& 0.86\end{array}$} & Spring '13 & 17 & 0 \\
\hline & & & & Autumn '14 & 14 & 0 \\
\hline & & & & Spring '14 & 18 & 1 \\
\hline & & & & Autumn '15 & 23 & 1 \\
\hline \multirow{2}{*}{ Whangaokeno } & \multirow{2}{*}{13} & \multirow{2}{*}{44} & \multirow{2}{*}{3.39} & Summer '14 & 14 & 1 \\
\hline & & & & Summer '15 & 8 & 0 \\
\hline \multicolumn{7}{|l|}{ Young Nicks Head } \\
\hline Adults & 35 & 42 & 1.2 & Summer '15 & 7 & 0 \\
\hline \multirow{2}{*}{ Juveniles } & \multirow{2}{*}{0.005} & \multirow{2}{*}{20} & \multirow{2}{*}{4,000} & Spring 2013 & 13 & 0 \\
\hline & & & & Summer '14 & 20 & 0 \\
\hline \multirow{4}{*}{ ZEALANDIA } & \multirow{4}{*}{225} & \multirow{4}{*}{200} & \multirow{4}{*}{0.89} & Spring '13 & 23 & 0 \\
\hline & & & & Autumn '14 & 24 & 0 \\
\hline & & & & Spring '14 & 19 & 0 \\
\hline & & & & Autumn '15 & 24 & 0 \\
\hline \multirow{2}{*}{ Stephens Island } & \multirow{2}{*}{150} & 30- & \multirow{2}{*}{$200-333$} & Autumn '14 & 35 & 1 \\
\hline & & 50,000 & & Autumn '15 & 43 & 0 \\
\hline
\end{tabular}




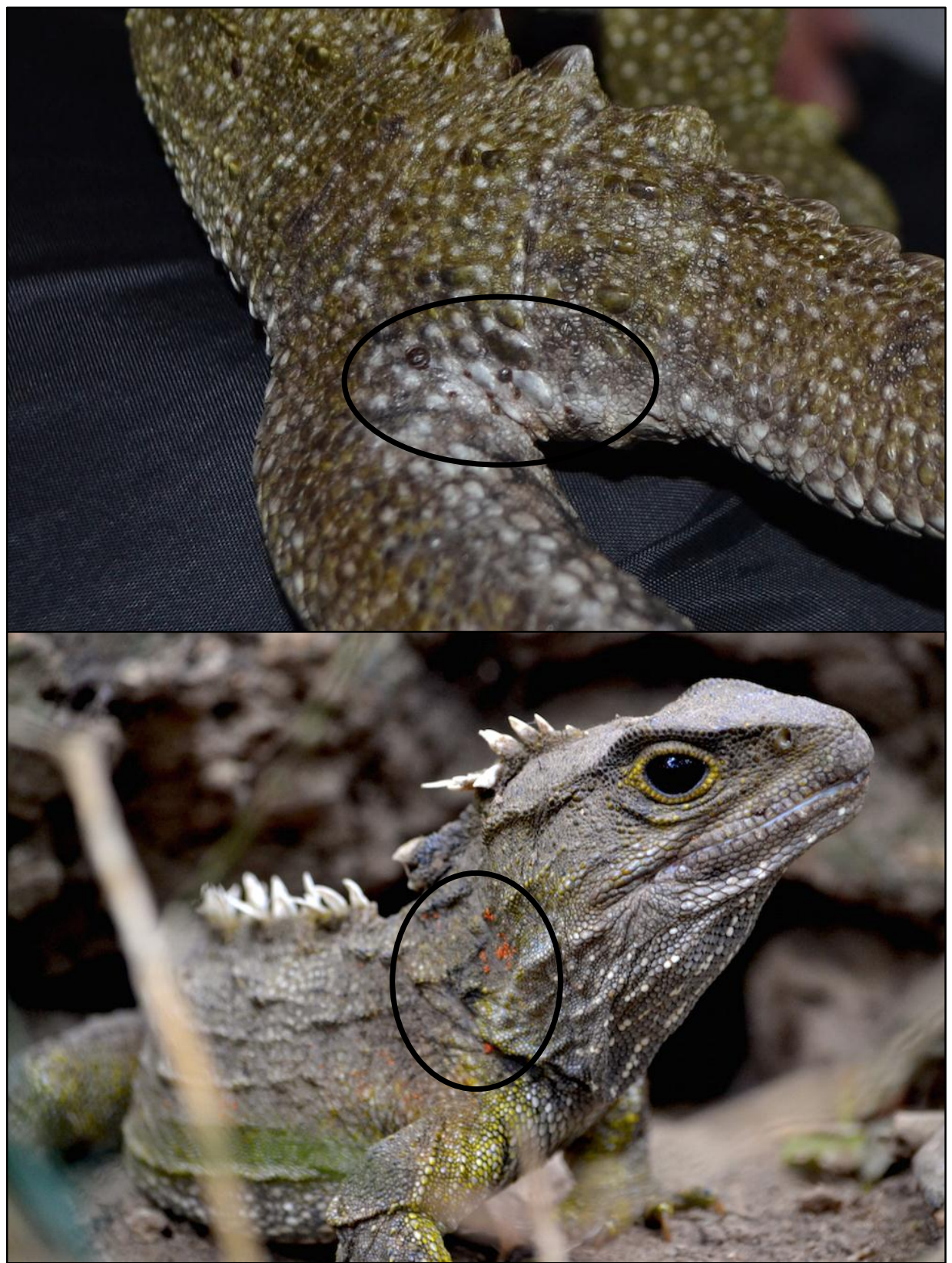

Figure 4.1. Photographs showing tuatara ticks (A. Sphenodonti) and trombiculid mites infesting tuatara. Top: cluster of tuatara ticks of different life stages visible in fork of adult tuatara's tail. Bottom: aggregations of red trombiculid mites visible along the side of an adult tuatara's neck. Ectoparasites are ringed in black circles to highlight locations. Photographs: S. Price.

\subsubsection{Sample analysis}

I conducted tick and mite counts in the field. Any faecal samples I obtained were transferred to cryovials and transported to the parasitology lab at the Institute of Vet, Animal \& Biomedical Sciences, Massey University, Palmerston North where I performed faecal floats. All samples arrived at the laboratory within 3-6 days of collection. Faecal 
floats were prepared by sieving $0.5 \mathrm{~g}$ of faeces into $33 \%$ zinc sulphate solution, which was transferred to a test tube with a cover slip set on top and centrifuged at 1,200 rpm for five minutes. The cover slip was then removed and placed on a slide for examination under a microscope (Sloss et al. 1994).

\subsubsection{Statistical analysis}

\subsubsection{Ectoparasite presence/absence}

Changes in prevalence following translocation (i.e. between October 2012 and November 2013 onwards) were investigated in the Cape Sanctuary and Maungatautari populations (the only translocation sites visited one year post-release) using generalised linear mixed effects models (GLMMs) with ectoparasite presence/absence as the outcome variable, survey as the fixed effect and individual ID as a random effect to account for the lack of independence in observations (as some individuals were repeatedly captured throughout the four post-release surveys). We used the package "Ime4" for generalised linear (GLM) and generalised linear mixed effects (GLMM) analyses (Bates et al. 2016). The levels of the variable "survey" were re-ordered so that the October 2012 survey data (pretranslocation) was the baseline against which the post-release survey presence/absence data were compared.

To assess factors associated with ectoparasite prevalence I ran separate GLMs with binomial distributions for each survey season to investigate ectoparasite presence/absence as a function of mean temperature, mean 9am relative humidity (RH), body condition (BCl), SVL, sex and population density (See Chapter Three, section 3.2.5 for population density calculation methods). I obtained values for mean temperature and mean $\mathrm{RH}$ by averaging monthly temperature/RH values for the 12 months prior to the survey. For instance, if a survey was conducted in March 2014, I calculated the mean of the monthly values for the 12-month period March 2013 to February 2014. Data were obtained using the NIWA (National Institute of Water and Atmospheric Research Ltd.) Cliflo database (http://www.cliflo.niwa.co.nz) using the meteorological stations in closest proximity to the translocation sites for which temperature and $\mathrm{RH}$ data were available. The suitability of models was compared using second-order Akaike information criterion $\left(A I C_{C}\right)$ value for small samples (used when the parameter to sample size ratio was less 
than 40 ( $\mathrm{n} / \mathrm{K}<40$; Burnham \& Anderson 2003). AIC $\mathrm{C}$ values were obtained using the $\mathrm{R}$ package "AICcmodavg" (Mazerolle 2016). The best-fit model was then compared to the null model using the models chi-squared statistic, which, if significant, indicated that the inclusion of predictors significantly improved the fit of the model. When constructing regression models I included one predictor variable per 10 data points where appropriate, therefore I did not perform GLMs on samples of fewer than 10 individuals. Body condition indices were calculated separately for each survey season as the residuals of a linear regression of log-transformed mass and log-transformed SVL, which has been used in previous studies on tuatara (Hoare et al. 2006; Moore et al. 2007; Godfrey et al. 2010a). The $\mathrm{BCl}$ indices used in this study met the assumption of linearity (spring $2013 \mathrm{R}^{2}=0.84$, autumn $2014 R^{2}=0.84$, spring $2014 R^{2}=0.84$, autumn $2015 R^{2}=0.82$ ).

Analyses were performed in R v. 3.2.2 (R Core Team 2015). Tick and mite data were assessed independently. Endoparasite prevalence was not statistically assessed due to low sample sizes (see Results). Surveys conducted in February (late summer) were incorporated into the autumn survey category with other sites visited around the same time. Due to differences in rearing and exposure opportunities post-release, the juvenile Young Nicks Head Sanctuary population was not included in analyses with adult tuatara. As there were repeat recaptures of individuals across surveys, the influence of site on ectoparasite presence/absence was assessed independently for each survey season using Fisher's exact tests for count data. If a significant difference was found between the sites within a survey season, the source of the difference was investigated by testing all pairwise comparisons between sites using $2 \times 2$ contingency tables and the Bonferroni correction method.

\subsubsection{Ectoparasite load}

To investigate changes in ectoparasite load over surveys post-translocation I ran separate GLMMs with Poisson distributions for the Cape Sanctuary and Maungatautari populations with ectoparasite load as the outcome variable, survey as the fixed effect and individual ID as a random factor. As I only had information on ectoparasite prevalence and not load prior to translocation, I was unable to compare parasite load pre and post-translocation. Sample sizes were not sufficient to allow GLMMs to be run for Whangaokeno and Young 
Nicks Head. GLMs with Poisson distributions were used to determine the influence of the variables site, mean temperature, mean relative humidity, $\mathrm{BCl}$, sex, population density and SVL on ectoparasite load within each survey season. Separate GLMs were also run per site per survey to more specifically investigate the influence of sex, $\mathrm{BCl}$ and $\mathrm{SVL}$ on ectoparasite load. Model fit was assessed using AIC values and the chi-squared statistic. Previous work by Godfrey et al. (2010a) examined whether peak tick load affected subsequent body condition by comparing tick load and body condition during peak infestation (September) with post-peak (November) body condition. As I did not conduct surveys during the peak-infestation period I did not consider our data to be suitable for such an analysis.

\subsection{Results}

\subsubsection{Tick prevalence}

Between $90 \%$ and $100 \%$ of adult tuatara allocated to each site were carrying ticks immediately prior to translocation from Stephens Island (Figure 4.2). Over the course of this study, the apparent prevalence of ticks varied between $25 \%$ and $100 \%$ per site and tick loads varied between one and $\sim 175$ ticks per infested individual (Figures 4.2 and 4.3), with the highest tick loads observed to be aggregated on to one or two of the sampled individuals at each site in each survey. None of the head-started juveniles at Young Nicks Head were infected with ticks on release and were not found to be carrying ticks during either post-release survey.

Within the spring 2013 and spring 2014 surveys, Fisher's exact tests showed no significant differences in tick prevalence among the three sites surveyed (spring 2013: $p=$ 0.21 ; spring 2014: $p=0.21$ ). However significant site-dependent differences in prevalence were found in autumn $2014(p=0.002)$ and autumn $2015(p<0.001)$. Pairwise comparisons with a Bonferroni correction showed that, in autumn 2014, the Whangaokeno population had significantly lower tick prevalence than the Stephens Island population (2014: p < 0.001). Similarly, in 2015 the Whangaokeno population had significantly lower prevalence than both the ZEALANDIA and Stephens Island populations 
(ZEALANDIA: $p=0.002$; Stephens Island: $p<0.001$ ) and prevalence in the Cape Sanctuary population was also significantly lower than the Stephens Island population $(p=0.001)$.

One year following release a substantial decrease in tick prevalence was observed in both the Cape Sanctuary and Maungatautari populations (Figure 4.2). GLMMs investigating the influence of survey on tick prevalence at these two sites (Cape Sanctuary: $\chi 2=10.649, p=0.031$; Maungatautari: $\chi 2=12.266, p=0.015)$ showed that tick prevalence approximately one year post-translocation had dropped significantly in comparison to pre-translocation prevalence (Cape Sanctuary: estimate $=-2.042 \pm 0.813$, $z=-2.511, p=0.012 ;$ Maungatautari: estimate $=-2.265 \pm 0.790, z=-2.867, p=0.004$, Figure 4.2). No significant difference was found between pre-translocation prevalence and prevalence in any of the subsequent surveys at either site $(p>0.05)$, though there appears to have been decreases in prevalence in spring 2014 and autumn 2015 at both sites (Figure 4.2). Neither Whangaokeno nor Young Nicks Head Sanctuary were surveyed in spring 2013 and post-translocation sample sizes were too small to allow for reliable analysis. However it is evident from the apparent prevalence data that tick prevalence in the Whangaokeno population dropped substantially following translocation (Figure 4.2). Conversely, tick prevalence in the Young Nicks Head Sanctuary population in autumn 2015 was higher than the tick prevalence documented at other translocation sites in the same season and was comparable to pre-translocation prevalence, though these data are based on a small sample size ( $n=7$, Figure 4.2). I also compared pre and post-translocation tick prevalences in the ZEALANDIA tuatara population and found that, after McKenzie (2007) recorded a significant decrease in prevalence one year post-release, tick prevalence has subsequently increased, but remained significantly lower than pre-translocation levels ( $\chi 2$ $=29.319, \mathrm{p}<0.001$, summer '05 prevalence vs. spring 2013, autumn '14, spring '14 and autumn '15 prevalences $=p<0.001$ ).

When assessing the influence of population density, temperature, humidity, sex, $\mathrm{BCl}$ and $\mathrm{SVL}$ on tick prevalence within each survey season we compared $\mathrm{AlC}_{\mathrm{C}}$ outputs from the GLMs to determine which models were better quality. None of the GLMs for the spring 2013 or spring 2014 survey data were better than chance (when compared to null models) at predicting tick prevalence and I was unable to discern any influential factors. The GLM that provided the best fit to the autumn 2014 survey data $(\chi 2=15.247, p<0.001$, Table 4.2) showed a significant relationship with mean temperature (for the prior 12 months; 
estimate $=-0.877 \pm 0.370, z=-2.371, p=0.018$ ) and population density (estimate $=64.317$ $\pm 32.029, z=2.008, p=0.045$ ) on tick prevalence. Sites that had experienced lower mean temperatures in the 12 months prior to the survey had comparatively higher prevalences (e.g. Stephens Island had a mean prior temperature of $13.48^{\circ} \mathrm{C}$ and $97 \%$ prevalence, whereas Whangaokeno had a mean prior temperature of $15.64^{\circ} \mathrm{C}$ and $53 \%$ prevalence), and the more densely populated sites showed higher tick prevalences (i.e. Cape Sanctuary, Maungatautari Tautari Wetland and Stephens Island, Figure 4.2). The GLM that provided the best fit to the autumn 2015 survey data ( $\chi 2=22.946, p<0.001$, Table 4.2) showed a significant relationship with mean temperature (estimate $=-1.437 \pm 0.460, z=$ -3.124, $p=0.002$ ) and mean RH (estimate $=0.106 \pm 0.047, z=2.233, p=0.026$ ) on tick prevalence, with a lower mean temperature and higher $\mathrm{RH}$ for the previous 12 months associated with higher prevalence. 


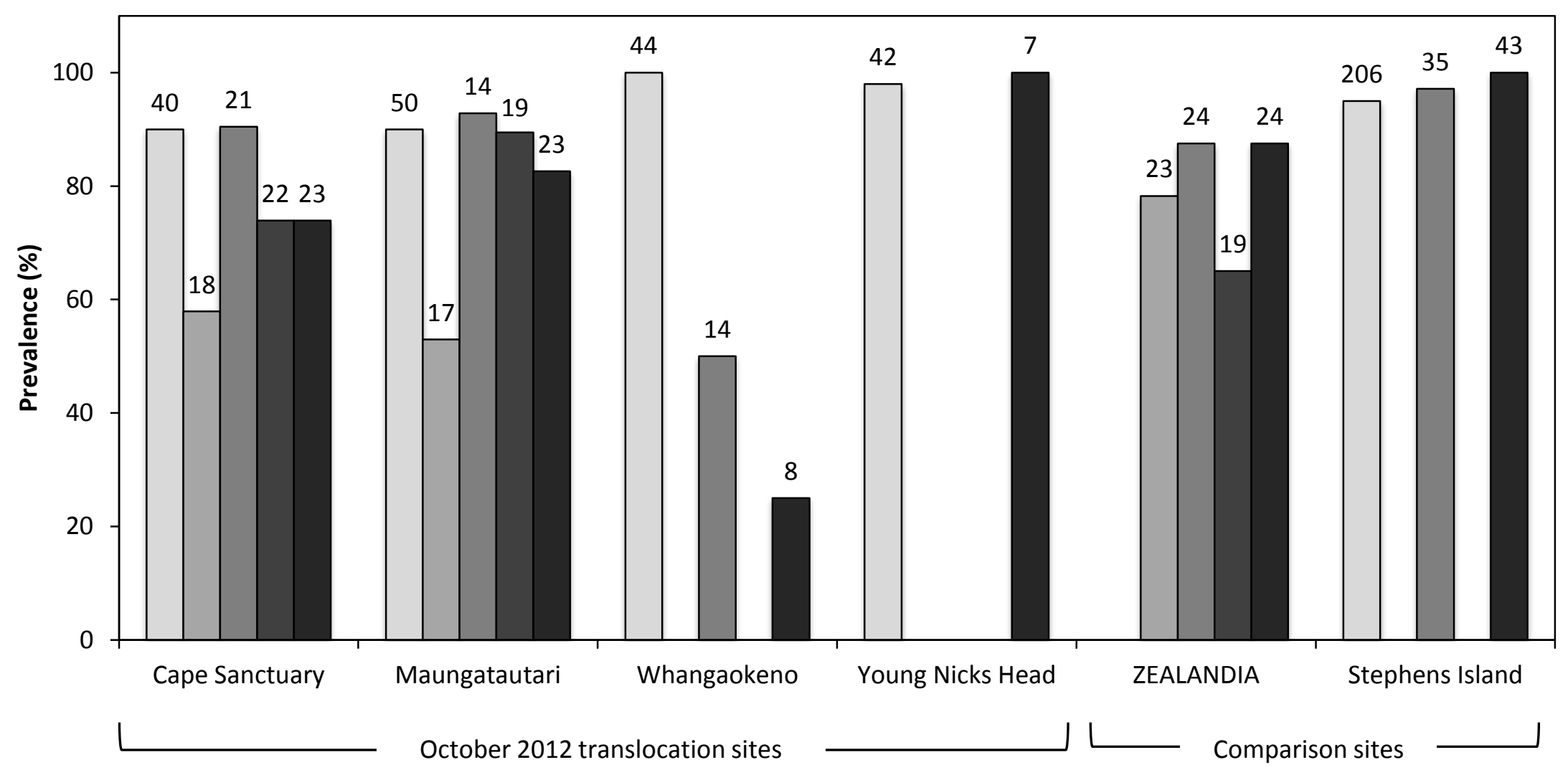

\section{$\square$ Spring '12 $\square$ Spring '13 $\square$ Autumn '14 $\square$ Spring '14 $\square$ Autumn '15}

Figure 4.2 Tick apparent prevalences for adult tuatara by survey per site. Data labels above bars show the sample size (i.e. number of adult tuatara examined). The spring '12 category shows pre-translocation prevalences per population. The spring '12 prevalence for Stephens Island was calculated using the total number of adult tuatara captured for translocation off the island, including adult tuatara caught for translocation to Orokonui Ecosanctuary. While Whangeokeno and Young Nicks Head populations were both surveyed in February (late summmer), their data have been grouped under autumn for comparison purposes 


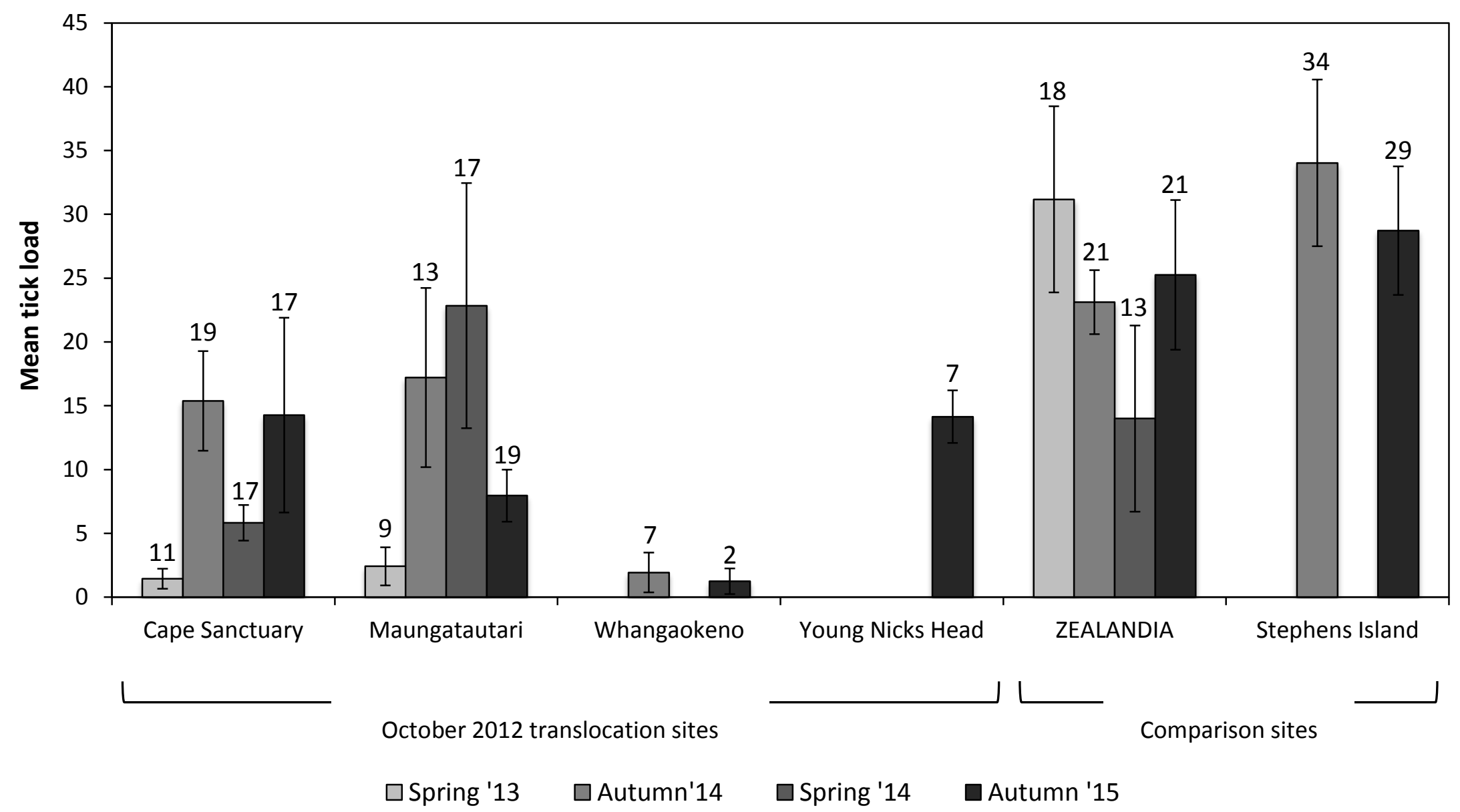

Figure 4.3 Mean ( \pm 1 std. error) tick load per survey per site. Data labels show the sample size, the number of adult tuatara captured that were carrying $\geq 1$ tick(s). While Whangaokeno and Young Nicks Head Sanctuary populations were both surveyed in February (late summmer), their data have been grouped under autumn for comparison. 
Table 4.2 Results of analysis of competing GLMs on factors associated with tick prevalence in the autumn 2014 and autumn 2015 survey seasons. $\mathrm{K}=$ number of estimated parameters in the model. $A I C_{C}=$ second-order Akaike information criterion. $\Delta\left(A I C_{C}\right)=\left[A I C_{C}-\min \left(A I C_{C}\right)\right]$. Relative likelihood $=\exp \left(-0.5^{*} \Delta\left(A I C_{C}\right)\right)$. w $\left(A I C_{C}\right)=w_{C}$ wight of the model. The null model AIC value has been included for comparison.

\begin{tabular}{|c|c|c|c|c|c|c|}
\hline Season/survey & Model predictor variables & K & $\mathrm{AlC}_{\mathrm{c}}$ & $\Delta\left(\mathrm{AIC}_{\mathrm{C}}\right)$ & $\begin{array}{c}\text { Relative } \\
\text { likelihood }\end{array}$ & $w\left(A I C_{C}\right)$ \\
\hline \multirow{9}{*}{ Autumn 2014} & Mean temperature + pop. density & 3 & 74.337 & 0 & 1 & 0.566 \\
\hline & Mean temperature & 2 & 76.544 & 2.207 & 0.332 & 0.188 \\
\hline & Mean temperature + pop. density + mean $\mathrm{RH}$ & 4 & 76.704 & 2.367 & 0.306 & 0.173 \\
\hline & Pop. density & 2 & 78.639 & 4.302 & 0.116 & 0.066 \\
\hline & Mean RH & 2 & 84.486 & 10.149 & 0.006 & 0.004 \\
\hline & Null model & 1 & 85.584 & - & - & - \\
\hline & $\mathrm{BCl}$ & 2 & 85.986 & 11.649 & 0.003 & 0.002 \\
\hline & Sex & 2 & 87.389 & 13.052 & 0.001 & 0.001 \\
\hline & SVL & 2 & 87.404 & 13.067 & 0.001 & 0.001 \\
\hline \multirow{9}{*}{ Autumn 2015} & Mean temperature + pop. density + mean $\mathrm{RH}$ & 4 & 92.896 & 0 & 1 & 0.732 \\
\hline & Mean temperature + pop. density & 3 & 96.135 & 3.239 & 0.198 & 0.145 \\
\hline & Mean temperature & 2 & 96.504 & 3.608 & 0.165 & 0.121 \\
\hline & Pop. density & 2 & 105.523 & 12.627 & 0.002 & 0.001 \\
\hline & Mean RH & 2 & 108.319 & 15.423 & 0.000 & 0.000 \\
\hline & Null model & 1 & 109.549 & - & - & - \\
\hline & $\mathrm{BCl}$ & 2 & 110.912 & 18.016 & 0.000 & 0.000 \\
\hline & SVL & 2 & 111.031 & 18.135 & 0.000 & 0.000 \\
\hline & Sex & 2 & 111.370 & 18.474 & 0.000 & 0.000 \\
\hline
\end{tabular}




\subsubsection{Tick infestation intensity}

I used GLMMs to assess changes in tick loads among infested individuals between surveys and found that, within three of the study sites, there was significant variation in tick loads over surveys (Cape Sanctuary: $\chi 2=274.740, p<0.001$, Maungatautari: $\chi 2=275.630, p<$ 0.001; ZEALANDIA: $\chi 2=122.440, p<0.001$, Figure 4.3). Significantly lower loads were observed in the populations monitored in spring 2013 (Cape Sanctuary and Maungatautari) when compared to loads in subsequent surveys (Table 4.3, Figure 4.3). I was not able to examine differences in tick loads between surveys for the Whangaokeno, Stephens Island or Young Nicks Head Sanctuary populations as the tick-positive sample size for Whangaokeno was too small (autumn 2014: $n=8$, autumn 2015: $n=2$ ), repeat recaptures could not be accounted for between Stephens Island surveys and the Young Nicks Head Sanctuary population was surveyed only once post-release. However, mean tick loads did not appear to vary substantially between the 2014 and 2015 surveys conducted on Stephens Island and Whangaokeno (Figure 4.3).

I used GLMs to assess the influence of mean temperature, mean RH, study site, SVL, sex and $\mathrm{BCl}$ on tick loads within survey seasons. I did not include the fixed effect site in the same models as mean temperature or mean $\mathrm{RH}$ as the study sites experienced different mean temperature/RH values I considered these factors to be confounded. I found that loads were significantly influenced by the factors mean temperature (over 12 months prior to the survey), site, sex, BCI and SVL (Table 4.3; Appendix 4, Table A4.1). Owing to the significant variation in infection intensity among surveys and the multiple influential factors involved, I decided to assess factors influencing tick load individually per site per survey. Due to small numbers of tick-positive individuals from the Whangaokeno and Young Nicks Head Sanctuary populations ( $\leq 8$ per survey), I were unable to assess factors influencing tick load at these sites.

The GLMs that provided the best fits to the tick load data for other sites found that no one factor was the dominant predictor for a particular site or seasonal survey, but that SVL, $\mathrm{BCl}$ and sex were all significant factors at different points throughout the study. Equally, the direction of the relationship between these factors and tick load was not consistent across sites and surveys (see Appendix 4, Table A4.2). 
Table 4.3 Results of GLMs comparing spring 2013 (one year post-release) tick loads at Cape Sanctuary and Maungatautari with loads observed in subsequent survey seasons.

\begin{tabular}{crrrrr}
\hline Site & \multicolumn{1}{c}{ Season } & estimate & \multicolumn{1}{c}{ Std. error } & \multicolumn{1}{c}{ z-value } & \multicolumn{1}{c}{ p-value } \\
\hline \multirow{3}{*}{ Cape Sanctuary } & Autumn 2014 & 2.049 & 0.211 & 9.710 & $<0.001$ \\
& Spring 2014 & 1.226 & 0.225 & 5.443 & $<0.001$ \\
& Autumn 2015 & 2.339 & 0.214 & 10.917 & $<0.001$ \\
\hline \multirow{3}{*}{ Maungatautari } & Autumn 2014 & 1.542 & 0.181 & 8.509 & $<0.001$ \\
& Spring 2014 & 2.022 & 0.176 & 11.516 & $<0.001$ \\
& Autumn 2015 & 0.935 & 0.190 & 4.937 & $<0.001$ \\
\hline
\end{tabular}

\subsubsection{Mite prevalence and infection intensity}

Immediately prior to translocation all captured tuatara were examined for ectoparasite infection and all were found to be free of mites. Over the four post-translocation surveys between November 2013 and April 2015 no mites were found on any of the translocated individuals, resulting in an apparent prevalence of zero per cent in these populations. Earlier work by McKenzie (2007) found that 33 (47\%) of the initial 70 tuatara translocated to ZEALANDIA in 2005 had mites on release, but surveys one month and up to one year post-release found no mites on recaptured animals. In the course of this study, no mites were found on any animals in the ZEALANDIA population, indicating that apparent prevalence has remained at zero per cent or very low.

Tuatara in the source population on Stephens Island were surveyed in March 2014 and 2015, at times of peak infestation (Godfrey et al. 2010a), and found to be infested with mites during both surveys. Mite loads of infected individuals varied between one and an estimated 775 mites. Twenty-four of 35 captured animals had mites during the March 2014 survey and 27 of 43 animals had mites during the March 2015 survey, equating to apparent prevalences of $69 \%$ and $63 \%$ respectively. No GLMs were significantly better fits to the mite prevalence data than the null model $(p>0.05)$, indicating that the predictor variables $\mathrm{BCl}$, sex and SVL were no better than chance at predicting mite presence/absence. 
Among infected tuatara on Stephens Island, mite loads varied between 11 and 600 in autumn 2014 and one and 775 in autumn 2015. Mean mite load among infected animals was 197 in 2014 and 247 in 2015, indicating some annual variability in the number of mites carried. I assessed factors influencing mite load within individuals found to be infected. Because there were 24 and 27 mite-positive individuals in the autumn 2014 and 2015 surveys respectively, our small samples did not allow us to include more than two predictor variables per model or look at interaction effects between variables. The GLM that provided the best fit to the autumn 2014 survey data $(\chi 2=943.756, p<0.001$, Table 4.4) found significant effects of body condition (estimate $=-2.995 \pm 0.107, z=-28.072, p$ $<0.001$ ) and sex (estimate $=-0.131 \pm 0.032, z=-4.087, p<0.001)$ on mite load. There was a significant negative association between mite load and $\mathrm{BCl}$ score, with poorer condition animals carrying more mites, and females were shown to have higher mite loads than males. The GLM that provided the best fit to the autumn 2015 survey data ( $\chi 2=330.439$, $p<0.001$, Table 4.4) showed significant positive correlations between mite load and body condition (estimate $=1.031 \pm 0.099, z=10.46, p<0.001$ ) and SVL (estimate $=0.008 \pm$ $0.001, z=12.80, p<0.001$ ), with larger animals and those in better condition found to be carrying higher numbers of mites during this survey.

Table 4.4 Results of competing GLMs on factors associated with mite load in the autumn 2014 and autumn 2015 survey seasons. $\mathrm{K}=$ number of estimated parameters in the model. $A \mathrm{AC}_{\mathrm{C}}=$ second-order Akaike information criterion. $\Delta\left(\mathrm{AIC}_{\mathrm{C}}\right)=$ $\left[A I C_{C}-\min \left(A I C_{C}\right)\right]$. Relative likelihood $=\exp \left(-0.5^{*} \Delta\left(A I C_{C}\right)\right) \cdot w\left(A I C_{C}\right)=$ weight of the model.

\begin{tabular}{|c|c|c|c|c|c|c|}
\hline Season & $\begin{array}{l}\text { Model } \\
\text { predictor } \\
\text { variables }\end{array}$ & K & $\mathrm{AlC}_{\mathrm{c}}$ & $\Delta\left(\mathrm{AIC}_{\mathrm{C}}\right)$ & $\begin{array}{l}\text { Relative } \\
\text { likelihood }\end{array}$ & $w\left(A I C_{c}\right)$ \\
\hline \multirow{7}{*}{$\begin{array}{l}\text { Autumn } \\
2014\end{array}$} & $\mathrm{BCl}+\operatorname{sex}$ & 3 & 3656.156 & 0 & 1 & 0.997 \\
\hline & $\mathrm{BCl}+\mathrm{SVL}$ & 3 & 3668.828 & 12.672 & 0.002 & 0.002 \\
\hline & $\mathrm{BCl}$ & 2 & 3670.311 & 14.155 & 0.001 & 0.001 \\
\hline & Sex + SVL & 3 & 4361.664 & 705.508 & 0.000 & 0.000 \\
\hline & Sex & 2 & 4447.948 & 791.792 & 0.000 & 0.000 \\
\hline & Null model & 1 & 4594.894 & - & - & - \\
\hline & SVL & 2 & 4596.846 & 940.690 & 0.000 & 0.000 \\
\hline \multirow{7}{*}{$\begin{array}{l}\text { Autumn } \\
2015\end{array}$} & $\mathrm{BCl}+\mathrm{SVL}$ & 3 & 4156.437 & 0 & 1 & 1 \\
\hline & Sex + SVL & 3 & 4174.317 & 17.880 & 0.000 & 0.000 \\
\hline & $\mathrm{Sex}+\mathrm{BCl}$ & 3 & 4175.080 & 18.643 & 0.000 & 0.000 \\
\hline & Sex & 2 & 4199.163 & 42.726 & 0.000 & 0.000 \\
\hline & SVL & 2 & 4264.041 & 107.604 & 0.000 & 0.000 \\
\hline & $\mathrm{BCl}$ & 2 & 4313.178 & 156.741 & 0.000 & 0.000 \\
\hline & Null model & 1 & 4481.994 & - & - & - \\
\hline
\end{tabular}




\subsubsection{Endoparasites found in faeces}

A total of seven faecal samples were obtained from four of the six sites over the course of this study. Unfortunately this small overall sample size prevents any formal analysis of variables influencing endoparasite load but the details of the individual results are presented below. No faecal samples were collected from tuatara at Young Nicks Head Sanctuary or ZEALANDIA (Table 4.1). Six samples were collected from clinically normal animals. Of these six samples, faecal floats detected nematode ova in four and unidentified coccidial oocysts in one. One of the samples obtained from a male at Maungatautari in autumn 2014 had no detectable endoparasites, but a second faecal sample obtained from the same male in autumn 2015 detected coccidial oocysts (Table 4.5). Two individuals had more than one type of nematode ova present. The nematode ova detected had morphology consistent with ascarid-type ova and measured $\sim 80 \mathrm{x}$ $50 \mu \mathrm{m}$, as well as possible oxyurid ova measuring $~ 70 \times 30 \mu \mathrm{m}$ (Figure 4.4). A possible oxyurid larva was also found in a sample from a Whangaokeno female (Table 4.5). The seventh sample was found beside a male tuatara during the autumn 2015 survey at Cape Sanctuary. The male was in poor physical condition so was taken to Wildbase Hospital, Massey University, Palmerston North, New Zealand, for treatment. Examination of the faecal sample found a high number of coccidial oocysts $(187,200$ oocysts/g of faeces) and a heavy ascarid burden (200 eggs/g of faeces), and the male was diagnosed with clinical coccidiosis (Figure 4.4). 
Table 4.5 Results of faecal floats for the seven samples collected between November 2013 and May 2015. Both faecal samples from Maungatautari were collected from the same individual male. The Cape Sanctuary male captured and sampled in autumn 2015 was in poor physical condition and found to have coccidiosis.

\begin{tabular}{llll}
\hline Season/survey & Site & Animal & Endoparasites \\
\hline Spring 2013 & Cape Sanctuary & Adult male & Ascarid-type ova \\
Autumn 2014 & Maungatautari & Adult male & - \\
Summer 2014 & Whangaokeno & Adult female & $\begin{array}{l}\text { Ascarid-type ova } \\
\text { Oxyurid-type ova } \\
\text { Possible oxyurid larva }\end{array}$ \\
Autumn 2014 & Stephens Island & Adult male & $\begin{array}{l}\text { Ascarid-type ova } \\
\text { Oxyurid-type ova }\end{array}$ \\
Spring 2014 & Cape Sanctuary & Adult male & $\begin{array}{l}\text { Ascarid-type ova } \\
\text { Autumn 2015 }\end{array}$ \\
Mutumn 2015 & Maungatautari & Adult male & Coccidial oocysts \\
& Cape Sanctuary & Adult male & $\begin{array}{l}\text { Ascarid-type ova } \\
\text { Coccidial oocysts }\end{array}$ \\
\hline
\end{tabular}

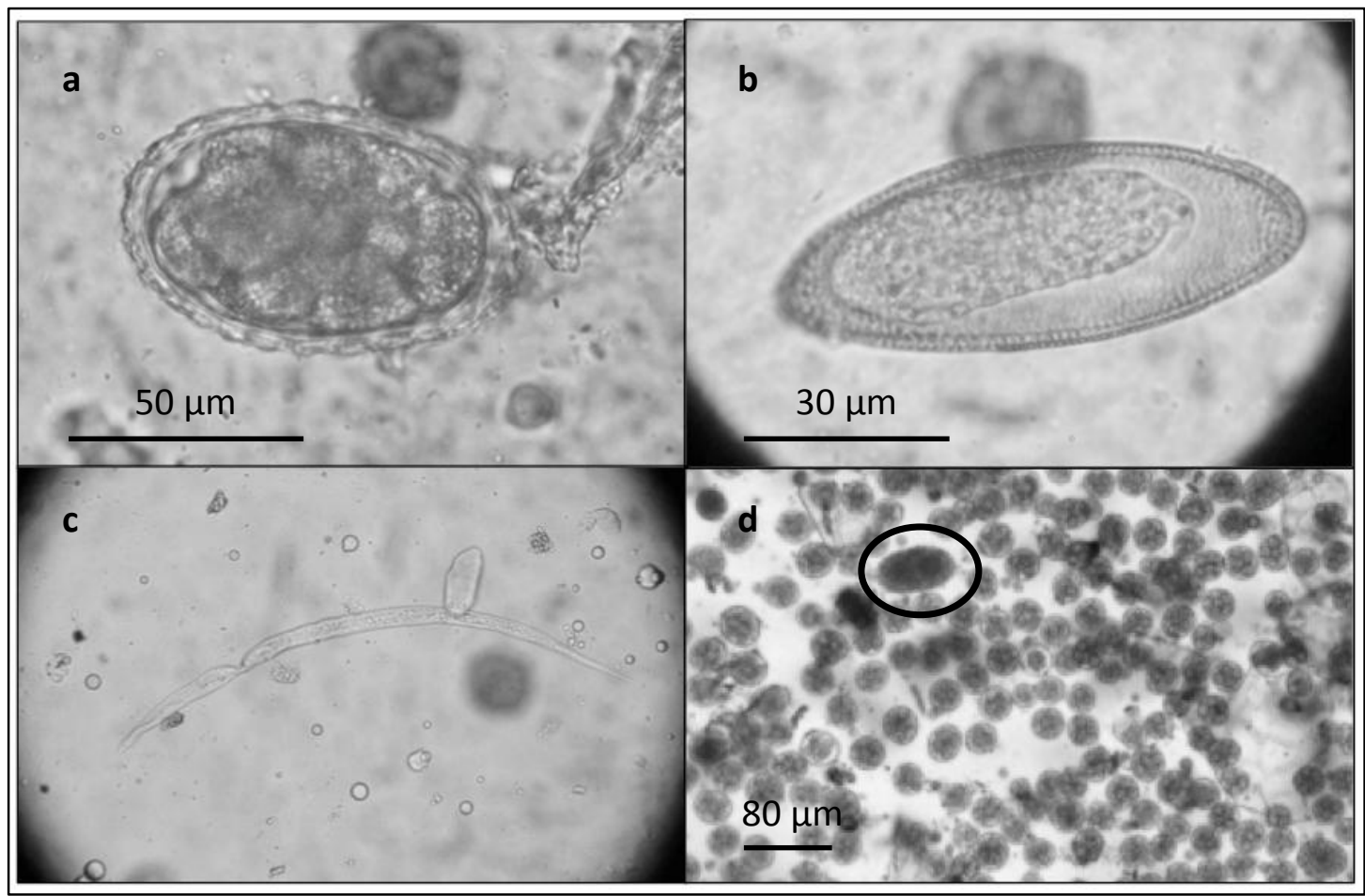

Figure 4.4 Parasitic organisms detected in faeces of adult tuatara by faecal float. (a) ascarid-type ova ( $80 \times 50 \mu \mathrm{m})$; (b) oxyurid ova ( $70 \times 30 \mu \mathrm{m})$; (c) Possible oxyurid larva; (d) high number of coccidial oocysts of varying sizes from male diagnosed with coccidiosis, ascarid-type ova also visible for scale (circled). 


\subsection{Discussion}

\subsubsection{Tick populations following translocation}

Significant reductions in tick prevalence following release and comparatively low spring 2013 tick loads in tuatara populations at Maungatautari and Cape Sanctuary illustrate a significant negative relationship between translocation and tick populations. Over successive surveys the prevalence at both sites was seen to recover to levels comparable to pre-translocation prevalence and tick loads were seen to increase. This demonstrates that the initial impact of translocation on tick populations did not persist over time, which indicates that factors associated with translocation of the host species were likely responsible for the decrease, rather than biotic or abiotic factors inherent to the different translocation sites. A similar pattern of decreased prevalence was observed following translocation of tuatara to ZEALANDIA in 2005 , with a $78 \%$ reduction in ticks prevalence observed in the year following translocation, which was attributed to low pre-existing parasite populations and low host population density at the release site (McKenzie 2007). Similarly, ticks were found to persist at very low abundances following the translocation of tuatara to Tiritiri Matangi Island (Moir et al. 2012) and decreased significantly following the translocation of wild-caught juveniles to Orokonui Ecosanctuary (Jarvie et al. 2015). Although prevalence data were not available for the ZEALANDIA population for the years immediately following the observed decrease, the data collected during this study shows that tick prevalence has since recovered significantly, though remaining below pretranslocation levels.

Due to the seasonal nature of the surveys undertaken in this study, I do not have data on the progression of observed declines in tick populations between release and the spring 2013 surveys. As such, low post-translocation numbers could be due to a failure to survive/remain attached to hosts during translocation (i.e. transport between sites), or it could be that conditions at the sites negatively affected tick survival following release. The more frequent data collected by McKenzie (2007) following the release of tuatara at ZEALANDIA (every five weeks in summer, spring and autumn) demonstrated a gradual overall decline in tick prevalence after release, indicating that ticks were able to survive the translocation itself, but populations went into decline after hosts were released at the 
site. It is therefore feasible that the tick populations monitored in this study also underwent gradual declines following host release.

Post-translocation dispersal of animals away from the release site has been cited as a prominent cause of translocation failure in reptiles (Germano \& Bishop 2008). Correspondingly, previous work on tuatara found that animals monitored for one year post-translocation showed infrequent reuse of burrows soon after release and dispersed an average of $41.14 \mathrm{~m}$, with one individual moving a total of $178.88 \mathrm{~m}$ away from the release burrow (McKenzie 2007). As tuatara are solitary, territorial reptiles it is likely that translocated animals would seek to establish new territories, refuges and resources following release, which could motivate this dispersal (Godfrey et al. 2010a). Transmission of $A$. sphenodonti is dependent upon direct contact with tuatara, with ticks thought to occupy the same environment as their hosts during free-living life stages, which should maximise the chances of survival and reattachment (Heath 2006; Kerr \& Bull 2006; Godfrey et al. 2011b). This sit-and-wait strategy is supported by the high prevalence of ticks observed over winter months, when tuatara spend most of their time dormant in burrows (Godfrey et al. 2008). Similar attachment patterns have been documented in the related reptile tick Amblyomma limbatum as well as Aponomma gervaisi, both of which show positive correlations between tick abundance and host burrow use (Auffenberg \& Auffenberg 1990; Kerr \& Bull 2006; Godfrey et al. 2008). As ticks are unlikely to travel far in search of a host, with $A$. limbatum found to move less than $500 \mathrm{~mm}$, close proximity to tuatara and repeated use of burrows is vital to tick survival (Petney et al. 1983). Tick loads on Stephens Island have been shown to decline between spring and summer as engorged ticks detach so following translocation in October, it is likely that ticks detached from their hosts not long after release (Godfrey et al. 2008). If engorged ticks detached in burrows from which hosts dispersed and did not return, the free-living ticks would be left unable to reattach and vulnerable to starvation, predation or unfavourable environmental conditions, resulting insubstantial decreases in tick populations. Limitations on dispersal distances could also offer explanations for the smaller prevalence decreases observed at Cape Sanctuary and Maungatautari when compared to the larger ZEALANDIA site at which tuatara would have been able to disperse greater distances, further reducing the likelihood of burrows being revisited (McKenzie 2007; Moir et al. 2012). 
An important concept to consider is that of a possible "dilution effect" in which, in some circumstances, higher host densities can be negatively related to host parasite load as parasites are spread out amongst the host population (Renwick \& Lambin 2013). Conversely, lower density host populations may carry comparatively higher parasite loads, as parasites are concentrated on to fewer available hosts. This effect has previously been observed in the Stephens Island tuatara population, with higher tick loads found on tuatara occupying the less densely populated pasture habitat when compared to animals living at higher densities in shrub and forest habitat (Godfrey et al. 2008). I found no evidence to suggest a possible dilution effect in our high versus low density translocated populations, however, it is possible that if it did occur it could have gone unnoticed and confounded our measures of parasite loss in the year following translocation. As such, the low loads observed at Cape Sanctuary and Maungatautari in spring 2013 could have been attributed to a spreading out of translocated ticks among the high-density hosts. In the absence of spring 2013 tick load data from the less densely populated Whangaokeno and Young Nicks Head Sanctuary sites I am unable to assess this; however, the subsequent recovery of tick prevalences and loads at Cape Sanctuary and Maungatautari, despite no change in density, suggests that this was not likely to have been the case.

Following the spring 2013 surveys, there was a substantial recovery of tick prevalence and increases in load at Cape Sanctuary and Maungatautari. I suggest that these increases can be attributed to a combination of tick biology, thermal climate, and population density. The significant association between population density and tick prevalence in the autumn 2014 survey suggests that the comparatively higher densities of tuatara at Maungatautari (specifically regarding the Tautari Wetland enclosure) and Cape Sanctuary may have facilitated increases in tick prevalence's and loads through increased transmission opportunities. The solitary-territorial nature of tuatara means that direct contact between individuals occurs primarily during aggressive interactions (e.g. territorial boundary defence) or mating, leaving little opportunity for direct parasite transmission (Stamps 1994; Stamps \& Krishnan 1998; Godfrey et al. 2010b). Consequently, transfer of parasites is considered reliant on indirect transmission of freeliving ticks via the asynchronous sharing of burrows and overlapping of undefended territorial boundaries (Mitani \& Rodman 1979; Godfrey et al. 2010b). I suggest that tuatara at the more densely populated sites may experience more territorial overlap (in 
both degree of overlap and number of overlapping neighbouring territories) and burrow sharing leading to more frequent contact between hosts and tick reservoirs, enabling more frequent transference of ticks. In accordance with this hypothesis, Godfrey et al. (2010b) found that tuatara social network in-strength (a measure of the number of territories that overlapped with a particular individual's territory, and the degree to which those territories overlapped) correlated positively with tick load in March (early autumn) and September (early spring), with strongly connected individuals found to carry higher total tick loads. Similarly, a study of parasite invasion following wolf (Canis lupus) reintroductions found that invasion of the sarcoptic mange-causing mite Sarcoptus scabiei was governed by population density and host connectivity, with the most densely populated areas also the most vulnerable to repeated parasite invasion and associated declines (Almberg et al. 2012).

In contrast, as the Whangaokeno and Young Nicks Head Sanctuary tuatara populations were not monitored in spring 2013, I am unable to confirm that they also experienced significant decreases in tick prevalence and load as a result of translocation. However, tick prevalence in the Whangaokeno population was significantly lower than pre-translocation levels during both post-translocation surveys and both prevalence's and loads were consistently significantly lower than those observed in the source population on Stephens Island. The high prevalence of ticks observed at Young Nicks Head Sanctuary in autumn 2015 was comparable to the pre-translocation prevalence, suggesting that the tick population could have followed a similar pattern of decline and recovery seen at other sites, or that prevalence and load could have remained unchanged following translocation. It is important to acknowledge the low capture numbers obtained at Young Nicks Head and Whangaokeno in autumn 2015, which may not be representative of the larger populations. However, given the clear effect of translocation on the Cape Sanctuary, Maungatautari and ZEALANDIA tick populations, I suggest that the tuatara released at Whangaokeno and Young Nicks Head Sanctuary likely experienced significant declines in tick prevalence and load in the year following translocation. Whangaokeno also provides a contrast to other translocation sites in that the prevalence's and loads documented were considerably lower than those seen in the other populations and at no point were comparable to pre-translocation levels, which suggests the presence of a limiting factor that prevented tick population recovery. 
I found that the thermal climate had a significant relationship with both tick prevalence and load in tuatara and may have influenced tick populations in multiple ways. Warmer temperatures have been found to initiate reproductive behaviour in ticks; therefore the rising spring temperatures may have stimulated mating among surviving adult $A$. sphenodonti (Chilton \& Bull 1991; Godfrey et al. 2008). The reproductive cycle of A. sphenodonti, from female engorgement to the appearance of larvae on hosts, has been found to take approximately three months (given average maximum summer temperatures of $\sim 18^{\circ} \mathrm{C}$ ), with larval tick loads in the Stephens Island tuatara population increasing between November and March (Godfrey et al. 2008). As warmer temperatures may both induce reproduction and enhance tick development this suggests that the warmer Cape Sanctuary and Maungatautari spring/summer climates (compared to Stephens Island) could have advanced reproduction and aided recovery of tick numbers at these sites, resulting in the significant increase observed in the spring 2013 survey, which took place in November. A northerly pattern of distribution has also been observed in other New Zealand tick species, likely due to the warmer climates experienced at lower latitudes. For instance, the New Zealand cattle tick (Haemaphysalis longicornis) tolerates a broad range of climates, but is most prevalent in the far north (Heath 2016).

Equally, while warmer spring/summer temperatures at translocation sites may have aided tick reproduction and recovery, I also found that the study sites that experienced warmer mean temperatures over the 12 months preceding the autumn 2014 survey had lower tick prevalence's than those that experienced a comparatively cooler mean temperature. While there is little information on the direct influence of air temperature on $A$. sphenodonti desiccation and survival, ticks are particularly vulnerable to desiccation when off-host, and display nocturnal activity patterns and preferences for humid, shaded substrates, which may be adaptations to reduce this risk (Oliver 1989; Needham \& Teel 1991; Godfrey et al. 2011b). Studies on other tick species have found that high relative humidity (>85\%) is required for survival of Ixodes ricinus and $I$. scapularis, with extended exposure to dry air leading to substantially reduced survival (Stafford 3rd 1994; Daniel et al. 1998; Rodgers et al. 2007). Similarly, survival of $I$. scapularis has also been shown to correlate negatively with air temperature and weight loss in unengorged $A$. americanum increases with temperature when maintained at low humidity (Sauer \& Hair 1971; Bertrand \& Wilson 1996). As relative humidity decreases 
with rising air temperature, the warmer mean temperature recorded for Whangaokeno for the 12 months prior to the survey suggests that the warmer climate at this site could have increased desiccation risk and impaired survival of ticks following translocation, thereby impeding tick population recovery and contributing to the prevalence differences observed (Godfrey et al. 2008). A similar relationship was found in the autumn 2015 survey season, in which lower mean temperatures and higher mean $\mathrm{RH}^{\prime} \mathrm{s}$ for the 12 previous months were associated with higher tick prevalence's. Although Whangaokeno did not have the lowest mean $\mathrm{RH}$, it was the warmest site, which could offer an explanation for the further decrease in tick prevalence observed in this translocated population. Additionally, the relationships between prior temperature, $\mathrm{RH}$ and tick loads observed in several surveys and the absence of clear associations between tick load and measures of $\mathrm{BCl}$, sex and SVL provides further evidence that the climate at a translocation site likely plays a key role in regulating tick populations.

As well as the effect of climate, I suggest that the low post-translocation tick populations observed on Whangaokeno are in part due to the site's size, the influence of which is illustrated in the greater prevalence drop observed at the large ZEALANDIA site when compared to smaller translocation sites. In comparison to Cape Sanctuary and Maungatautari, tuatara released on Whangaokeno would have been able to disperse further from release sites and less likely to revisit areas in which engorged ticks had detached, resulting in a greater decline in tick numbers following translocation. Following this, the low host population density and consequently limited transmission opportunities, in conjunction with temperatures unfavourable to off-host survival, could have hindered tick recovery. It is also worth noting that habitat differences could have contributed to the declining tick populations observed at Whangaokeno. The higher tick prevalence and mean load observed at the larger, less densely populated and climatically similar Young Nicks Head Sanctuary site appears to contradict the proposal that these factors were responsible for the low tick populations on Whangaokeno. However, there is variability in habitat structure. Extensive replanting has restored a closed-canopy forest to Young Nicks Head Sanctuary, which may provide a more favourable microclimate to free-living ticks, whereas Whangaokeno has patches of regenerated native vegetation and large areas of soft, bare soil and open grassland extensively colonised with seabird burrows (Thorsen 2004). It is therefore feasible that habitat-associated microclimatic 
conditions could have affected survival of free-living ticks and contributed to the lack of tick population recovery seen on Whangaokeno (Godfrey et al. 2011b). However, in contrast to this explanation, Godfrey et al. (2008) found that tick prevalence on Stephens Island was unaffected by habitat type.

\subsubsection{Mite populations following translocation}

There does not appear to have been a direct effect of translocation on mite load at any of the sites where tuatara were translocated in October 2012. In the Stephens Island population, mites are only present on tuatara in the austral summer and autumn, and tuatara were translocated from this site in spring, while no mites were attached. As such, it cannot be said that the act of translocation or the warmer climates of the translocation sites affected mite populations; only that translocation may have indirectly eradicated mites and reduced parasite diversity by removing uninfected tuatara to sites where they were not reinfected, a process that has been termed "missing the boat" (MacLeod et al. 2010). However, $47 \%$ of the tuatara translocated to ZEALANDIA in summer 2005 had mites on release, but were found to be free of mites one month later and none were detected up to one year post-release (McKenzie 2007). In the course of this study I found no mites on any tuatara in the ZEALANDIA population though high mite prevalence's and loads were observed in the Stephens Island population during both autumn surveys. The sustained absence of mites at ZEALANDIA in contrast to the source population indicates that translocation of hosts can cause a decrease in mite prevalence, potentially due to reduced host population density and impaired mite survival at the translocation site (McKenzie 2007).

Parasites that moved with the host but failed to persist at the new location can be referred to as "lost overboard" and a number of mechanisms have been proposed to explain post-translocation failure of parasite populations (MacLeod et al. 2010). Parasite populations can be lost due to the absence of a required host species, the insufficient transmission of parasites among hosts due to parasite-associated or host-associated transmission limitations (e.g. high host specificity, small founding populations, limited interactions between hosts), or due to the translocation of a small number of parasites with limited distribution among the host population, making the parasites more 
vulnerable to local extinction through stochastic events (Paterson et al. 2003; Torchin et al. 2003; MacLeod et al. 2010; Poulin 2011).

Despite the presence of alternative hosts (skinks and/or geckos) at translocation sites, that tuatara have not been infected with mites post-release indicates that $N$. sphenodonti and $N$. naultini may be absent from local reptile populations, or that necessary transmission routes between the different species/hosts were not established or were insufficient to enable mites to persist. In conjunction with the post-translocation decline observed at ZEALANDIA, a study by MacLeod et al. (2010) found that the loss of ectoparasitic lice in non-native birds following arrival in New Zealand could be attributed to failure of the parasite/host to establish populations or to transmission limitations. However, it is important to note that the inability to detect mites on the captured individuals is not definitive evidence that there is no mite infection in these populations, only demonstrative of zero prevalence in the animals sampled. Nonetheless, as mite load has been found to correlate negatively with tuatara body condition, which can ultimately affect reproductive success, the removal of mites following translocation may have longterm benefits for translocated populations (Godfrey et al. 2010a).

The inconsistent associations between body condition, SVL, sex and mite load in the Stephens Island surveys suggest that our sample size may have been too small to provide adequate analysis or that an alternative predictor not accounted for in these models may have had a more consistent and significant influence on mite load in this population. Previous work on mite transmission also found significant associations between host sex and size, but also found that territory size and habitat type was significantly associated with mite load (Godfrey et al. 2008, 2010b). As mite transmission is reliant on tuatara coming into contact with infective larvae in the environment, it is logical that the size of an individual's territory might influence the likelihood of contacting locations where larval mites have hatched (Godfrey et al. 2010b). Although I documented the habitat in which individuals were captured during nocturnal surveys, as tuatara have been observed to move substantial distances from burrows overnight (Chapter Five), I could not reliably identify an individual's home range and therefore habitat type for statistical comparison. 


\subsubsection{Occurrence of intestinal endoparasites}

That only seven faecal samples were obtained from multiple tuatara populations over multiple surveys demonstrates the practical difficulties faced in obtaining sufficient sample sizes for reliable analysis from tuatara held for short periods of time. I was able to identify two types of nematode ova, a potential nematode larva, and coccidial oocysts in multiple samples from clinically normal translocated tuatara. One individual found in poor physical condition at Cape Sanctuary and transported elsewhere for medical treatment was found to be heavily infected with oocysts of the protozoa Coccidia and was diagnosed with coccidiosis. This animal subsequently recovered after treatment with a coccidiocidal drug (toltrazuril) and was re-released at Cape Sanctuary. No other animals were found in similarly poor condition at this site. Our limited data set prevents any analysis of factors associated with intestinal parasite carriage following translocation to warmer climates and I am unable to speculate whether these parasites were translocated with tuatara or acquired following release. However, these findings are consistent with and add to previous work on gastrointestinal parasites of tuatara. Failure to identify the parasite ova observed to a more specific degree than genus classification demonstrates that further research is still needed on endoparasite carriage in this species (Gartrell et al. 2006).

\subsubsection{Conclusions and conservation implications}

As ectoparasite prevalence has been found to negatively affect body condition and can adversely impact fitness and survival in reptiles and other taxa, it could be argued that declines in parasite populations following translocation are beneficial to host species and therefore desirable (Scott 1988; Brown et al. 1995; Moller \& Nielsen 2007; Godfrey et al. 2008, 2010a; Almberg et al. 2012). However, these negative impacts can also be important to host population regulation, preservation of genetic diversity, and the resilience of populations to novel environmental challenges and it has been argued that parasite diversity should be considered as a conservation target in its own right (Hudson

\& Greenman 1998; Godfrey et al. 2008, 2010a; Almberg et al. 2012; Moir et al. 2012; Gómez \& Nichols 2013; Reisinger et al. 2015). 
The trombiculid mites that infect tuatara are not listed as threatened or at risk, as they are not reliant on tuatara as hosts, and their absence following translocation should not be considered a particular concern to parasite conservation (Buckley et al. 2012). On the other hand, the host-specific tuatara tick is considered at risk and relict, like its host, and has been found to occupy a more severely restricted distribution, with low genetic diversity within island populations (Miller et al. 2007; Buckley et al. 2012; Hitchmough et al. 2016). The absence of $A$. sphenodonti from islands with low densities of tuatara (i.e. < 1 tuatara per $10,000 \mathrm{~m}^{2}$ ) suggests that host density has played a key role in tick population survival and highlights their vulnerability to host population declines and extinctions (Miller et al. 2007). Although reduced host density following translocation has been highlighted as a potential risk to the maintenance of tick populations, our data suggest otherwise, demonstrating initial density-related declines post-release, followed by substantial recovery at most sites (Miller et al. 2007). However, only long-term monitoring will determine if these tick populations are able to persist at the translocation sites, but evidence of tick population recovery 10 years after release at ZEALANDIA is indicative of long-term survival post-translocation. As such there is little evidence that host translocation poses a significant threat to the conservation of $A$. sphenodonti.

Our data demonstrate significant short-term reductions in ectoparasite populations following translocation from Stephens Island and shows that removal of uninfected individuals to sites lacking in local ectoparasite reservoirs can effectively eradicate these parasites. Changes in prevalence post-translocation suggests that tick survival following release is determined by dispersal of tuatara away from the release site, with larger sites enabling further dispersal and greater declines in tick prevalence. However, it is possible that following these translocations I had confounding effects occurring with, on one hand, dispersal of hosts limiting re-attachment opportunities, while reduced host densities (compared to the source site) meant more ticks per individual host. These conflicting mechanisms may have made it difficult to determine total parasite numbers at the translocation site as opposed to our measure of parasites per host. However, given subsequent recovery of tick prevalence/loads I consider the declines observed to be due to host dispersal rather than dilution effects. I propose that density-dependent transmission and local climate are responsible for the subsequent recovery of tick populations, with a higher prevalence of ticks found at more densely 
populated translocation sites and a lack of recovery observed at the more sparsely populated, warmer Whangaokeno site (Arneberg et al. 1998; Godfrey et al. 2008). Experimental work would be required to further investigate these mechanisms and determine causal relationships; however, the highly protected status of tuatara limits the potential for manipulative experiments. Thus observational data provides the best available information on the interaction between this parasite and its host.

As anthropogenic climate change is predicted to facilitate continued increases in air temperature, parasite populations at the warmer translocation sites will likely be the first to encounter increasingly unfavourable temperatures or climatic effects (e.g. increased frequency of drought) that could influence off-host survival (IPCC 2014; Reisinger et al. 2014). Similarly, rising temperatures and decreased humidity could lead to thermal stress and immunosuppression in tuatara, which could facilitate higher parasite loads (Teixeira et al. 2007; Godfrey et al. 2008; Dickens et al. 2010). As evaporative water loss is a key constraint on tuatara activity (Hill 1982), reduced humidity could also impact tuatara behaviour, restricting activity outside of the burrow, increasing in-burrow transmission opportunities and facilitating increased tick loads, which could be detrimental to body condition, survival, and ultimately population viability (Hill 1982; Godfrey et al. 2008, 2010a; Corkery et al. 2014a). As I found evidence that temperature and humidity were significantly associated with host-parasite interactions I suggest that further work is needed to determine how parasite and tuatara populations might be affected as climate change progresses. It is also yet to be determined if biotic factors like habitat structure might offer some degree of buffering against increasingly warmer, drier climates, which could improve the survival of ecologically important parasites like $A$. sphenodonti resulting in changes to the currently stable host-parasite dynamic. Equally, further work on the ecology of free-living $A$. sphenodonti and the species temperature/RH tolerance thresholds under natural conditions could help inform future tuatara translocations decisions and parasite conservation strategies. 


\section{Chapter Five}

\section{Thermoregulatory behaviour and nocturnal activity in translocated populations of a cold-adapted reptile, the tuatara (Sphenodon punctatus): conservation implications with climate change}

\subsection{Introduction}

The optimal temperature and thermal sensitivities of enzymes, cell membranes, and aerobic respiration result in thermal boundaries for biological reactions from the cell to organismal level, beyond which an organism will cease to function normally (Angilletta 2009). Consequently, the biological functions of an organism (i.e. growth, digestion, reproduction, locomotion) are dependent on the maintenance of suitable body temperatures (Angilletta 2009). Endothermic taxa like mammals and birds are able to maintain thermal homeostasis through metabolic processes, buffering them against suboptimal environmental temperatures (Lowell \& Spiegelman 2000; Boyles et al. 2011). Conversely, reptiles and other ectothermic taxa are dependent upon suitable environmental temperatures and appropriate behaviours to effectively regulate body temperature and maintain normal physiological function, making them more sensitive to changes in environmental temperature (Deutsch et al. 2008).

The rate at which anthropogenic climate change is advancing is a significant conservation concern. IPCC models predict that global air temperatures will likely increase by $0.3^{\circ} \mathrm{C}$ to $4.8^{\circ} \mathrm{C}$ by the $2100 \mathrm{~s}$ (based on highest and lowest estimates from the CMIP5 model simulations RCP2.6 and RCP8.5; IPCC 2013). Associated environmental impacts are expected to have substantial effects on biodiversity (Parmesan 2006; IPCC 2014). Ectothermic taxa, like reptiles, may be especially affected by the rising air temperatures predicted under climate change as their physiological and ecological performance is dependent on environmental temperature (Huey \& Kingsolver 1989). It has been proposed that ectothermic species at mid to high latitudes might experience initial fitness benefits from temperature increases due to their broader thermal tolerances and the occupation of climates generally below their thermal optima (Deutsch et al. 2008). However, it has also been suggested that ectotherms living at mid to high latitudes could be more likely to experience thermal stress owing to the higher temperature increases 
predicted for these regions, overall lower tolerances to high temperatures, and limited potential for acclimation of their critical thermal limits (Calosi et al. 2008; Hoffman \& Sgro 2011; Hoffman et al. 2013). Nonetheless, as the climate continues to warm environmental temperatures will likely approach the thermal tolerance limits of even the most robust species and, in order to survive, animals will have to buffer themselves against temperature increases and mitigate the threat of extirpation/extinction though dispersal, adaptation, acclimation or behavioural adjustments (i.e. thermoregulation; Deutsch et al. 2008; Hoffman \& Sgro 2011; Quintero \& Wiens 2013).

In order to regulate body temperature, reptiles typically employ a suite of behavioural strategies that enable effective exploitation of the thermal environment. These behaviours include: 1) selection of suitable microhabitats (e.g. movement between sun, shade and shelter); 2) restriction of activity (e.g. periods of inactivity when temperatures are unsuitable); and 3) changes in posture and body shape (e.g. orientation towards a heat source; Huey \& Pianka 1977; Huey 1982; Stevenson 1985a; Losos 1987; Kearney \& Predavec 2000). Although the thermal environment can be substantially heterogeneous, some reptiles are capable of precise thermoregulation, keeping their body temperature within a narrow optimal range, while others are considered thermoconformers, active across a broad range of body temperatures (Kearney \& Predavec 2000; Besson \& Cree 2010). Although behavioural mechanisms can be effective, an organism's ability to maintain its preferred body temperature is ultimately dependent upon the presence of a heat source and the thermal quality of the environment, which is influenced by factors like solar radiation, humidity, habitat and geographical location, with reptiles living in temperate climates less likely than tropical species to experience their preferred body temperature (Huey 1974; Saint Girons 1980; Besson \& Cree 2010; Boyles et al. 2011; Corkery 2012). Reptiles in poorer quality environments (those with few thermally favourable sites) have been found to spend more time and energy on thermoregulation, which can reduce the amount of time available for other beneficial behaviours like mating, foraging, territory defence and predator avoidance (Huey 1974, 1982; Huey \& Slatkin 1976; Seebacher 1999).

The tuatara (Sphenodon punctatus) is a long-lived, nocturnal, New Zealand endemic reptile and the sole extant representative of the Order Rhynchocephalia (Saint Girons 1980; Barwick 1982; Dawbin 1982; Cree \& Butler 1993). Tuatara are considered to 
be a cold-adapted species, with a recorded preferred body temperature range of 19.5$23.1^{\circ} \mathrm{C}$ (Besson \& Cree 2010, 2011; Corkery et al. 2014a), and an active body temperature range of $5-30^{\circ} \mathrm{C}$ (Barwick 1982; Walls 1983; Thompson \& Daugherty 1998), although wild tuatara have been found to attain high body temperatures of up to $34.5^{\circ} \mathrm{C}$ for short periods of time (Corkery 2012) and captive tuatara have been observed to suffer heat stress and death at air temperatures above $25^{\circ} \mathrm{C}$ (Blanchard 2002). Holocene subfossil records indicate that tuatara were once widespread throughout New Zealand (Chapter One, Figure 1.1), but with the introduction of mammalian predators they were restricted to predator-free offshore islands (Cree \& Butler 1993; Anderson 1996; Holdaway \& Worthy 1997). Conservation translocations have since served to extend their current range, but tuatara are still considered to be conservation dependent, range restricted and relict (Miller et al. 2012; Hitchmough et al. 2016; Cree 2014). Their cold adapted lifestyle and limited dispersal ability places tuatara at risk from rising air temperatures under climate change, but also makes them an ideal species for studying the early effects of climate warming on reptiles (Nelson et al. 2002b; Mitchell et al. 2008).

The accuracy with which tuatara maintain preferred body temperatures has been debated in the past, with some suggesting that they actively thermoregulate whereas others have found limited evidence of this, suggesting that the species is more thermoconformatory (Saint Girons 1980; Saint Girons et al. 1980; Barwick 1982; Walls 1983; Thompson \& Daugherty 1998). An extensive study by Corkery (2012) found that tuatara on Stephens Island had the capacity for thermoregulation, but that the majority of individuals did not take advantage of opportunities to reach their preferred body temperatures when environmental temperatures allowed, demonstrating individuals in this population to be thermoconformatory most of the time. Conversely, Besson and Cree (2010) found captive juvenile tuatara tested under controlled laboratory conditions to be active thermoregulators regardless of habitat thermal quality, and were able to modulate their thermoregulatory effectiveness in response to quality of the thermal environment, achieving higher body temperatures and thermoregulating more precisely when in lowquality habitat. Similarly, captive juvenile tuatara have been seen to adjust their behaviour from diurno-nocturnal at higher temperatures to strictly diurnal emergence at low air temperatures $\left(5^{\circ} \mathrm{C}\right.$; Vermunt et al. 2014). However, whether observations of thermoregulatory behaviour made in captivity are applicable to wild populations, which 
may experience additional limiting factors, is debatable (Corkery 2012). Nonetheless it does illustrate that tuatara are capable of adjusting their thermoregulatory behaviour in response to thermal constraints (Besson \& Cree 2010). Thermoregulation in tuatara also appears to be influenced by habitat structure, with tuatara rarely observed basking in exposed areas, instead remaining under canopy and close to burrow entrances or choosing protected retreats when no canopy is present (Werner \& Whitaker 1978; Gans 1983; Corkery 2012). Apparent avoidance of open areas could be a response to habitatinfluenced microclimates, or, considering that predators of tuatara were historically avian, electing to bask under canopy/shelter could be a predator avoidance behaviour (Huey 1991).

Recent translocations of tuatara from Takapourewa/Stephens Island in the Cook Strait to four sites around New Zealand's North Island have served to establish tuatara populations at sites within their historical range (Chapter One, Figure 1.1). The comparatively warmer, drier climates of several regions to which tuatara were translocated, which are on average $2-4^{\circ} \mathrm{C}$ warmer during summer months, provide surrogates for temporal climate change, enabling an assessment of the ability of tuatara to adjust to a warming climate (Chapter Two, Figures 2.1 and 2.2). There are both benefits and risks associated with translocating tuatara from a comparatively cool source site like Stephens Island to locations with warmer climates. Tuatara are capable of year round activity, though this is dependent on environmental conditions and they are known to enter periods of inactivity when temperatures are low, generally remaining in their burrows over winter (Walls 1983). As tuatara emerge earlier from burrows when air temperatures are warmer, movement to warmer climates could lift this environmental constraint and enable more frequent, prolonged periods of activity on a daily and annual scale (Hughes 1967). Similarly, as thermoregulation is more costly in cooler climates, movement to an environment of higher thermal quality could see a reduction in time and energy spent thermoregulating, allowing more time for other fitness-related activities (Huey 1974, 1982; Huey \& Slatkin 1976; Seebacher 1999; Besson \& Cree 2010). Other benefits of increased environmental temperatures may include enhanced locomotor function, which could improve anti-predator response, foraging ability and territory defence, and increased lifetime reproductive output due to comparatively earlier sexual maturity owing to enhanced temperature-dependent growth rates (Christian \& Tracy 
1981; Avery et al. 1982; Castanet et al. 1988; Autumn \& De Nardo 1995; Besson \& Cree 2011).

Conversely, lower relative humidity and higher air temperatures at some translocation sites could increase evaporative water loss from the skin (Chapter Two, Figures 2.1 and 2.2). This could not only lower body temperature and influence thermoregulatory behaviour, but as tuatara lose a substantial amount of water through their skin and water is required to maintain body weight, it could also constrain the amount of time that can be spent outside of the burrow, reducing overall activity and negatively impacting fitness-related behaviours (Bogert 1949; Hill 1982; Barwick 1982; Corkery et al. 2014a). The importance of evaporative water loss as a constraining factor could be intensified at sites that are drought-prone or have no natural water source.

In this study I aimed to evaluate the different thermal and behavioural opportunities available to tuatara at different sites. To do this I assessed the different thermal climates available at study sites and used field-based observational and tracking methods to investigate differences in nocturnal activity and diurnal thermoregulatory behaviour among populations, and the dependence of these behaviours on temperature. My hypothesis was that tuatara translocated to warmer sites might experience increased behavioural opportunities (e.g. feeding, mating) throughout the year, which might confer behavioural and physiological benefits to these populations. This would imply potential fitness-related benefits with increasing air temperatures predicted under climate change and would likely influence future translocation management.

\subsection{Methods}

\subsubsection{Founder and translocation sites}

In October 2012, 176 adult tuatara were translocated from Takapourewa/Stephens Island in the Cook Strait to four sites around New Zealand's North Island: Maungatautari Ecological Reserve, Whangaokeno/East Island, Cape Sanctuary and Young Nicks Head Sanctuary (Chapter One, Figure 1.1). These translocations were designed to benefit conservation goals and all animals were released at protected, pest-free sites within their historical range (Worthy \& Holdaway 2002; Wood 2009; IUCN/SSC 2013). Stephens Island, 
the source site, is a pest-free, 150 ha offshore island nature reserve in the Marlborough Sounds and is a site of high conservation significance (East et al. 1995; DOC 2006). Cape Sanctuary, Young Nicks Head Sanctuary and Maungatautari are all fenced mainland sanctuaries located in New Zealand's North Island. Whangaokeno/East Island is the only unfenced site to which tuatara were translocated, but its location $\sim 2 \mathrm{~km}$ offshore places it beyond the swimming distance of introduced mammalian pests (King 1990; Nelson et al. 2002).

Forty adults and 20 head-started juvenile tuatara were released into two separate 2,000 $\mathrm{m}^{2}$ enclosures within a separately fenced, 1.5 ha site at Cape Sanctuary known as the Seabird Cell. Young Nicks Head Sanctuary received 44 adults (released into the fenced 35 ha sanctuary) and 20 head-started juveniles, which were released into a smaller $5 \times 10$ $\mathrm{m}$ enclosure also within the fence. Head-started juveniles were hatched from wildsourced eggs and raised in captivity until release. Whangaokeno also received 44 adults, which were released at three locations around the island while 50 adults were translocated to Maungatautari, with 30 released into a 35 ha enclosure on the mountain and 20 released into a $900 \mathrm{~m}^{2}$ enclosure in a separately fenced area known as the Tautari Wetland.

The tuatara population at ZEALANDIA ${ }^{\mathrm{TM}}$ in Wellington was also monitored for comparison purposes throughout this study. This sanctuary is located at the same latitude as Stephens Island and experiences a similar thermal climate, enabling a comparison of the effect of translocation, rather than climatic differences. This fenced sanctuary is home to 200 adult tuatara, which were translocated from Stephens Island in 2005 and 2007 (See Chapter Two section 2.2.1 for further detail on the source site and translocation sites). Due to access limitations imposed by landowners and permits, not all sites could be visited in every season. Of the October 2012 translocated populations, tuatara released at Young Nicks Head Sanctuary and Whangaokeno were not behaviourally monitored in this study.

\subsubsection{Habitat thermal quality}

I assessed the ambient environmental temperatures to determine the thermal quality of each translocation site. To record environmental temperature I fastened one temperature datalogger (Onset HOBO Pro v2 External Temperature/Relative Humidity 
Data Logger, Onset, MA, USA; reported accuracy $\pm 0.21^{\circ} \mathrm{C}$ ) to a tree $\sim 1.5 \mathrm{~m}$ off the ground at each site, ensuring that the sensor was positioned out of direct sunlight. These dataloggers also recorded relative humidity $(\mathrm{RH})$. However, due to consistently irregular $\mathrm{RH}$ readings obtained by several of these dataloggers during their time in the field all $\mathrm{RH}$ readings were considered unreliable, unfortunately excluding $\mathrm{RH}$ as a factor in analyses performed in this study. The thermal climates of the study sites were compared by calculating the sum of the degree days per site per month. Unfortunately, the air temperature dataloggers at Cape Sanctuary and Maungatautari failed in August 2014 and did not record air temperature data for August, September or October so degree days could not be calculated for these months, but it is a reasonable assumption that these two sites followed similar patterns of month-to-month degree day variation observed at the other sites. Datalogger failure is also the reason that regional temperatures from weather stations, rather than site-specific data, are represented in Figure 2.1 (Chapter Two). Site-specific air temperature data were obtained from $\mathrm{HOBO}$ dataloggers on site for the majority of sites between April $1^{\text {st }} 2014$ and February $18^{\text {th }} 2015$ so degree days were assessed over this time period. I assessed degree days in relation to the upper limit $\left(>25^{\circ} \mathrm{C}\right)$ and lower $\left(<10^{\circ} \mathrm{C}\right)$ air temperature threshold for burrow emergence and tuatara preferred body temperatures $\left(>19^{\circ} \mathrm{C}\right.$; upper and lower thresholds were based on results from this study as well as prior research and threshold estimates; Blanchard 2002; Mello et al. 2013). A degree day was calculated as the number of degrees by which a day's maximum recorded air temperature exceeded the selected baseline air temperature so it is a representation of whether or not a day exceeded the baseline temperature as well as how much it was exceeded by (e.g. for a baseline temperature of $10^{\circ} \mathrm{C}$, a maximum temperature of $15^{\circ} \mathrm{C}$ would equate to a five degree day and a maximum temperature of $9^{\circ} \mathrm{C}$ would equate to a zero degree day). Degree days were then added together to calculate the total number of degree days per month. This integrated measure combines both the number of days per month that the sites thermal climate exceeded a selected baseline temperature, as well as the number of degrees by which this baseline was exceeded. Some degree day calculations make use of hourly rather than daily measurements, which could have provided a more detailed measure of thermal availability throughout the day, however, results of hourly degree day measurements would likely have been highly correlated with daily degree day measurements when 
assessing thermal availability per site, and so I consider the output from a daily measure of degree days to be an equally useful representation of site specific thermal climates. This method of degree day calculation provides us with an approximate estimate of the availability of thermal climates at each site that are influential to the thermoregulatory behaviours of interest (e.g. emergence from or restriction to burrows) and the opportunity to attain body temperatures within the estimated preferred range. As such, this approach can be used to inform the thermal opportunities likely to be available at a site and help us predict potential challenges and benefits for a translocated population.

I also placed five temperature dataloggers in natural burrows at ZEALANDIA and Stephens Island, artificial burrows at Maungatautari (e.g. buried plastic containers) and both natural and artificial burrows at Cape Sanctuary (e.g. bore holes, buried terracotta pipes). The burrows into which dataloggers were placed were representative of the burrows available and in use by tuatara at each site at the time of installation. Data loggers were programmed to record temperature every 30 minutes and were left in the field for up to 2.5 years (November 2013 - May 2015) and checked periodically. The temperatures recorded in the five burrows at each site were averaged to obtain a range of mean, minimum and maximum burrow temperatures per site. It was assumed that tuatara could obtain a body temperature anywhere in the range between those recorded in burrows and those recorded in the environment. However it is possible that tuatara would have been able to attain higher body temperatures if they basked in patches of direct sunlight (which have been found to reach $40^{\circ} \mathrm{C}$ on Stephens Island; Corkery 2012), though it is not likely that all tuatara would have had the opportunity to do so due to home range distributions and habitat structure, which could have restricted access to patches of sunlight.

iButtons (Thermochron iButton ${ }^{\circledR}$ model DS1921G, Embedded Data Systems, KY, USA; $17.5 \times 6 \mathrm{~mm}, 3.26 \mathrm{~g}$; reported accuracy $\pm 1^{\circ} \mathrm{C}$ ) were attached to the behaviourally monitored adult females (see nocturnal activity methods) to record the range of skin temperatures obtained at each study site and were averaged across all monitored females per site to obtain a mean tuatara skin temperature per hour. iButtons were positioned at the base of the tail and attached with surgical tape, which was camouflaged with damp soil to reduce its visibility. The benefit of attaching the iButtons using surgical tape was that, should the female not be recaptured during subsequent surveys, the tape and 
iButton would eventually drop off into the environment when the tape became sufficiently wet/damaged or the animal moulted. However this also meant that iButtons could fall off during the behavioural monitoring period. Care was taken to place the iButton sufficiently behind the female's rear legs that it would not affect movement, but not so far down the tail that the tape might get caught on items in the environment and be pulled off or hinder the animal. iButtons were set to record skin temperature once every hour.

Externally attached iButtons have been shown to record accurate representations of internal body temperature across a narrow range of body temperatures (within $\pm 0.3^{\circ} \mathrm{C}$ of internal thermocouple-obtained body temperatures across $18.5-23.6^{\circ} \mathrm{C}$; Corkery 2012). However, in the absence of experimental tests of iButton accuracy across a broader range of body temperatures, I will consider the data obtained by externally attached iButtons to be inexact measures of body temperature, and will refer to the data obtained as "skin temperature" for the rest of this chapter. It is possible to record internal body temperature data by surgically implanting iButtons in the intraperitoneal cavity of an individual; however, given the protected status afforded to tuatara this was not considered politically feasible. Equally, the retrieval of externally attached devices is a much more practical and less invasive procedure, especially as the recapture of individuals can be difficult, and the use of a remote device negates the need for repeated capture and measurement using a handheld rectal thermometer or infra-red thermometer, which could elevate body temperatures (via handling of the animal) or disturb behavioural observations (Corkery 2012).

To compare the range of skin temperatures achieved by female tuatara at each of the four sites where iButtons were attached, I calculated the average skin temperature per hour per site for the month of May 2014. I chose to monitor adult females as their behaviour was thought to be more reflective of abiotic/biotic influences, whereas male behaviour would also be influenced by territoriality (Gillingham et al. 1995). Though it is important to acknowledge that female thermoregulatory behaviour could have been affected by reproductive condition, which would not be consistent across years (i.e. females reproduce every four years on average). I assessed only May 2014 data as I was only able to obtain skin temperatures from all sites for this time period due to differing dates on which the iButtons were attached to females, their limited storage capacity, and 
the loss of several iButtons due to drop-off in the environment. From this I calculated the mean daily skin temperature, the minimum mean daily skin temperature, and the maximum mean daily skin temperature. The range of daily skin temperatures was then compared among sites using Kruskal-Wallis tests and post hoc pairwise comparisons were conducted using Mann-Whitney $U$ tests. To determine if any observed differences in skin temperature might be due to the different air temperatures experienced at the study sites I ran Spearman's rank-order correlations to assess the correlation between mean tuatara skin temperature and air temperature (obtained from on-site $\mathrm{HOBO}$ dataloggers) in May 2014.

\subsubsection{Nocturnal activity}

The female tuatara populations at Cape Sanctuary, Maungatautari and ZEALANDIA were behaviourally monitored over two post-translocation surveys between November 2014 and May 2015 (Table 5.1). Due to visitation restrictions, the Stephens Island population was only behaviourally monitored once (March 2015). Tuatara were captured at night and had multiple measurements taken as part of a larger survey of the translocated populations (Chapter Two). Due to the opportunistic nature of tuatara captures, some individuals were captured only once during this study whilst others were captured and monitored in both the spring 2014 and autumn 2015 surveys. Recaptures were particularly high at Maungatautari where only eight females were present in the Tautari Wetland enclosure, thus the same six females were captured and monitored in both surveys. Recapture of individuals can be problematic if animals are recaptured due to behavioural or physiological traits (i.e. more active, less responsive to disturbance, gravid) that differentiate them from the wider population. However, I consider the repeat captures of females to be an effect of the small female populations at each site and the location of the female's home ranges within the areas studied (i.e. monitoring at the 225 ha ZEALANDIA site was focussed around the Research Area and adjacent tracks, so repeat capture of females inhabiting this area was likely). Equally, as I monitored reasonably large proportions of the female populations (with the exception of ZEALANDIA - Table 5.1) I consider it likely that the females monitored were representative of the wider female population. 
Overnight activity was measured in adult female tuatara over two survey seasons (spring 2014 and autumn 2015, Table 5.1). Study sites were visited for a total of 10 days, with up to 10 females captured on the first night (extended to the second or third night if initial captures were low) and monitored for up to seven consecutive nights. Following the collection of morphometric data, females were fitted with a temperature datalogger (as described in habitat thermal quality methods) and a spool of white cotton thread (38 $x 10 \mathrm{~mm}, 4.41 \mathrm{~g})$, which were attached to opposite sides of the base of the tail using surgical tape (Figure 5.1). The attachment of cotton spools faced similar problems as the attachment of iButtons, in that they could drop off into the environment if the female moulted or the surgical tape became too wet/damaged. However, this also meant that the cotton spool would eventually drop off if the female was not recaptured. There was also a risk of losing the ability to track females if the cotton thread broke. In this event, care was taken to remove loose thread from the environment to prevent entanglement.

The free end of the cotton spool was fastened to a $\sim 13 \mathrm{~cm}$ flat head nail marked with reflective bicycle tape, which was driven into the ground at the females capture location to act as an anchor for the thread (Figure 5.1). The female was then released and the capture location marked with neon flagging tape labeled with the female's individual ID to enable reliable identification of the "spooled" female without the need for recapture. The following morning, capture locations were revisited and the unspooled thread (released from the cotton spool as the female moved away from the nail) followed to find the female's new location. The new location was marked with flagging tape, the thread cut and retied to a new nail, and the female left undisturbed. The cotton spools of females were cut and re-tied by $\sim 1000-1100$ hours depending on the number of females being monitored and the distance/complexity of nocturnal movements. Following daytime observations (see thermoregulatory behaviour methodology) all cotton spools/nails were reset (excess cotton from any diurnal movement was collected and the spool retied to the nail) between 1730 and 1800 hours. This was usually done outside of the burrow/shelter that the female occupied during the day. The process was repeated up to a total of seven consecutive nights. Data from an individual's first night (immediately post-capture) was not recorded, as it was not representative of undisturbed nocturnal activity, meaning I obtained a maximum of six consecutive nights worth of data from each individual. Each morning I recorded emergence (whether or not a female had emerged 
from the refuge), displacement (the greatest distance moved from the burrow), and net distance moved (measured as the total length of the unspooled cotton), measured with a retractable tape measure (Figure 5.2).

All statistical analyses were performed in R v. 3.2.2 (R Core Team 2015). I used the package "Ime4" to perform linear mixed effects and generalised linear mixed effects analyses (Bates et al. 2016). Nocturnal activity data from the spring 2014 and autumn 2015 survey seasons were assessed separately to account for the influence of seasonal factors (i.e. mating behaviour). I compared the spring 2014 mean nocturnal temperatures experienced at each study site using a Kruskal-Wallis test to see if the nocturnal thermal climates differed significantly during the period that nocturnal activity was monitored. If a significant difference was found between sites, post hoc testing was done using MannWhitney $U$ tests. The autumn 2015 mean nocturnal temperature data were assessed in the same way. I constructed linear mixed effects models to examine nocturnal activity as a function of mean overnight temperature, population density and site. I used net distance and displacement as outcome variables and mean temperature, population density and site as fixed effects without interaction terms. Individual ID was used as a random factor to control for repeat measures (as individuals were repeatedly observed over multiple nights). I visually assessed residual plots for normality and homoscedasticity and saw no obvious deviations from normality or homogeneity of variance. P-values were obtained using likelihood-ratio tests (using the "anova" function in R) of the full model with the effects in question against models without those effects (i.e. null and reduced models). Kruskal-Wallis tests were used to determine between-site differences in net distance and displacement. To investigate the effect of overnight temperature on emergence of tuatara from burrows I used a generalised linear mixed effects model (GLMM) with a binomial distribution and modelled emergence as the outcome variable, mean overnight temperature as the fixed effect and individual ID as a random effect to control for repeat measures. I also used a separate GLMM to investigate differences in emergence between sites using emergence as the outcome variable and study site as a fixed effect. I considered the fixed effects site and mean overnight temperature to be confounded (as sites experienced a different range of nocturnal temperatures) so these factors were not included in the same model. The suitability of models was compared using second-order Akaike information criterion $\left(A I C_{C}\right)$ values for small samples (used 
when the parameter to sample size ratio is less than $40(n / K<40 ;$ Burnham \& Anderson 2003). AIC C values were obtained using the R package "AICcmodavg" (Mazerolle 2016). To assess where tuatara moved overnight I looked at observations for animals that had emerged and calculated the percentage of total observations that saw the individual return to the same location or move to a different location following emergence.

Table 5.1 Information on sites where behavioural monitoring took place, tuatara population size and sex, population density, survey season, number of adult female tuatara monitored in each season as number and proportion of adult female population. Seasonal sampling was classified as follows: spring: November-December; autumn: March-May. Population density estimates for Stephens Island vary with total estimated population sizes of 30,000 and 50,000 (see Chapter 3, section 3.2.5 for details). Information only presented for Maungatautari Tautari Wetland population as the main mountain population was not involved in the behavioural monitoring due to difficulty with access. Proportion of adult females monitored at Stephens Island unknown as total adult female population unknown.

\begin{tabular}{|c|c|c|c|c|c|}
\hline Site & $\begin{array}{c}\text { Pop. size \& } \\
\text { sex }\end{array}$ & $\begin{array}{l}\text { Pop. density } \\
\text { (no. per ha) }\end{array}$ & $\begin{array}{l}\text { Survey } \\
\text { season }\end{array}$ & $\begin{array}{l}\text { No. females } \\
\text { monitored }\end{array}$ & $\begin{array}{c}\text { \% of female } \\
\text { pop. }\end{array}$ \\
\hline \multirow{2}{*}{ Cape Sanctuary } & 40 & \multirow{2}{*}{200} & Spring '14 & 10 & 50 \\
\hline & $(20 \mathrm{M} ; 20 \mathrm{~F})$ & & Autumn '15 & 9 & 45 \\
\hline \multirow{2}{*}{$\begin{array}{l}\text { Maungatautari } \\
\text { Tautari Wetland }\end{array}$} & 20 & \multirow{2}{*}{222} & Spring '14 & 6 & 86 \\
\hline & (12 M; $8 \mathrm{~F})$ & & Autumn '15 & 6 & 86 \\
\hline \multirow[b]{2}{*}{ ZEALANDIA } & 200 & \multirow[b]{2}{*}{0.89} & Spring '14 & 6 & 6 \\
\hline & $\begin{array}{c}(104 \mathrm{M} ; \\
96 \mathrm{~F})\end{array}$ & & Autumn '15 & 9 & 9 \\
\hline Stephens Island & $30-50,000$ & $200-333$ & Autumn '15 & 10 & - \\
\hline
\end{tabular}




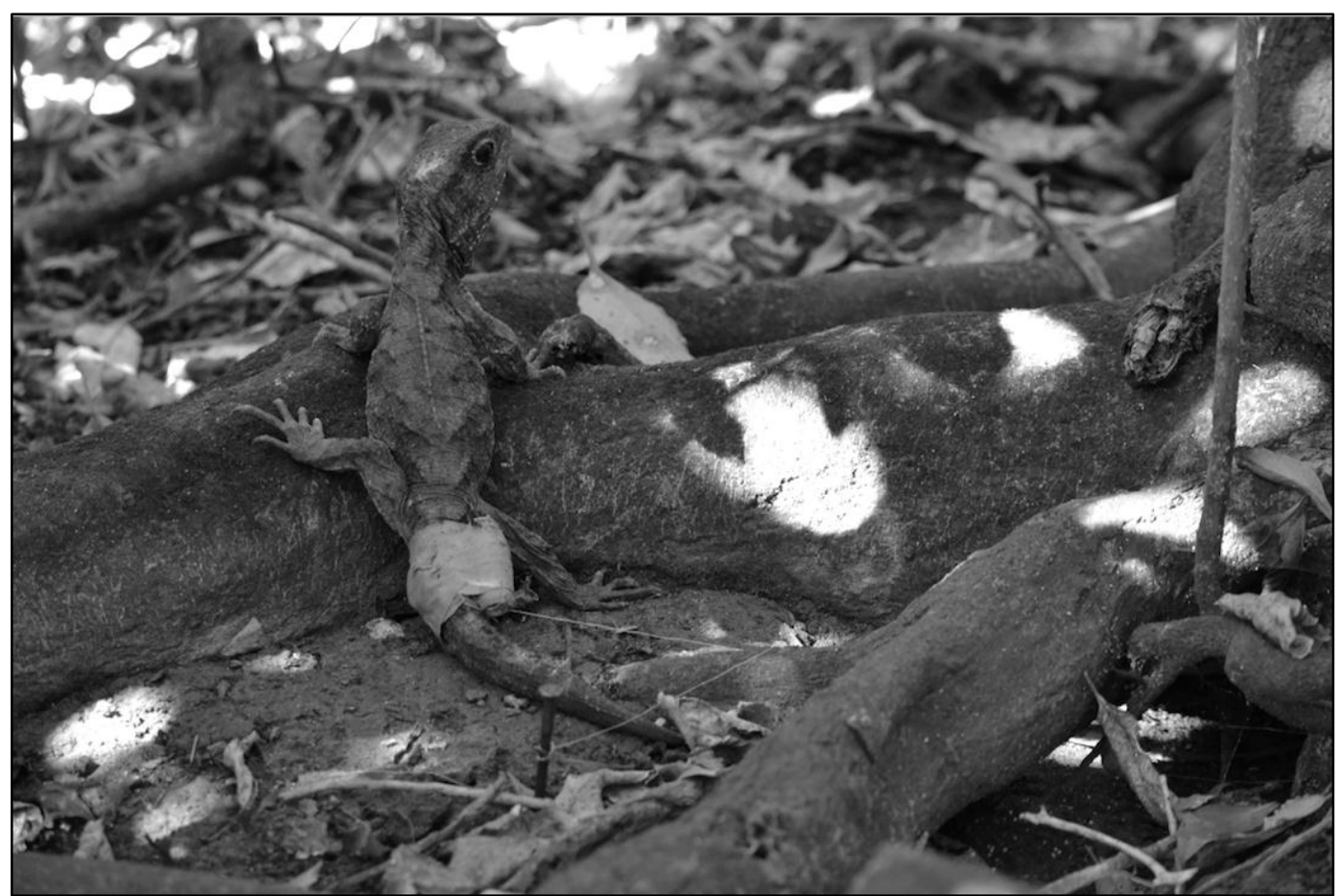

Figure 5.1 Adult female tuatara with attached iButton and cotton spool. The cotton spool is attached to the right side of the tail and the outline of the iButton can be seen beneath the surgical tape on the left. Nail "anchor" is visible in the foreground and string can be seen leading into and out of the burrow.

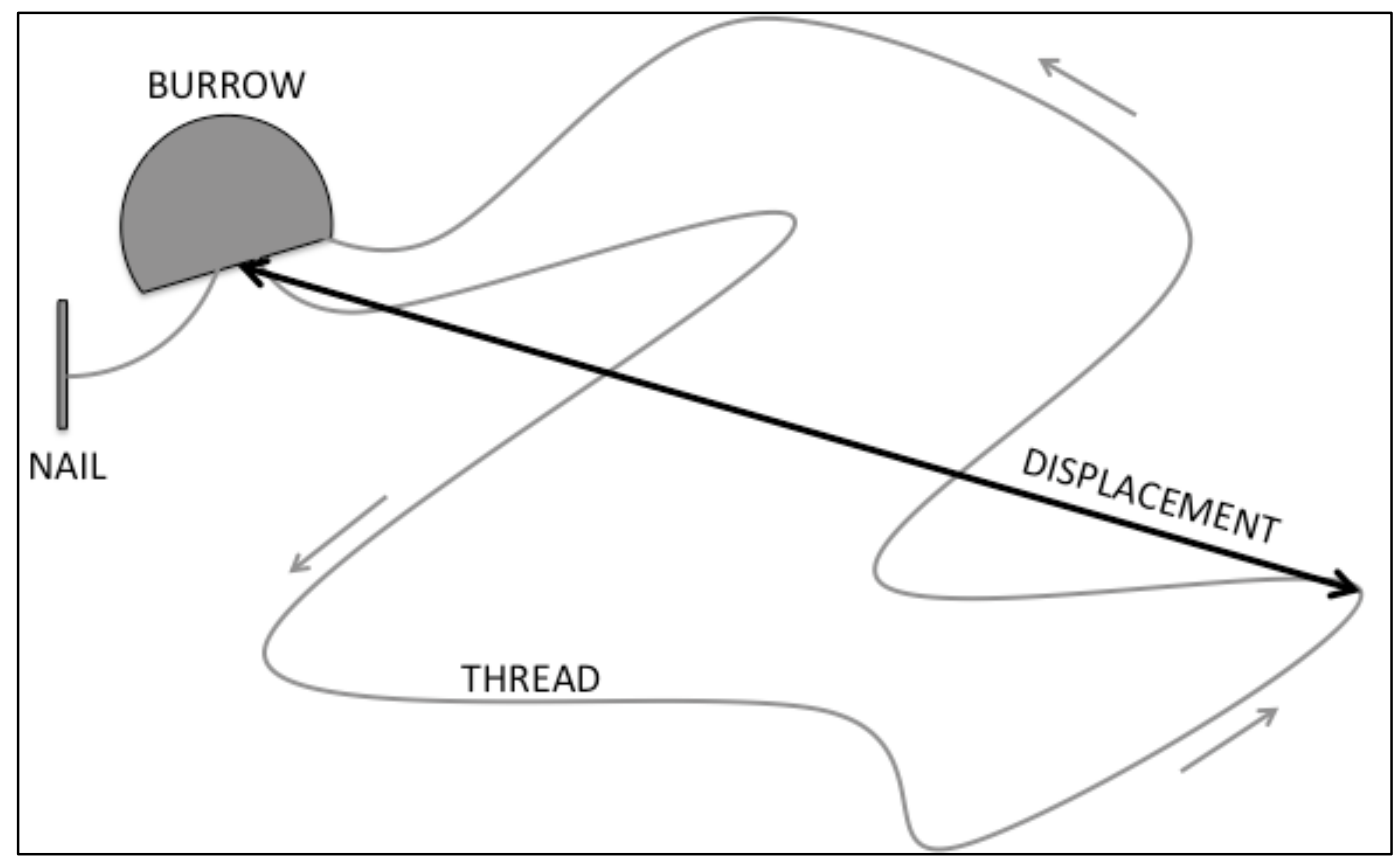

Figure 5.2 Diagram illustrating monitoring setup and measurements of nocturnal activity. Displacement = maximum distance moved from start point (e.g. burrow). Net distance = total length of unspooled cotton string. Evidence of string leaving burrow indicates nocturnal emergence of tuatara. Grey arrows indicate direction of animal's movement in environment. 


\subsubsection{Thermoregulatory behaviour}

During daylight hours, females that had been captured and "spooled" were periodically observed to document their thermoregulatory behaviour. Observations were made every two hours at 1100, 1300, 1500 and 1700 as the observer walked a track though the site and position of the female in the environment was recorded. It took approximately 20-40 minutes (depending on the site) to observe all of the females. The observer was careful to keep noise minimal and observations were made from a distance where possible to minimise disturbance, though maintaining distance was not always feasible due to complex vegetation obstructing the view of the observer. Females were categorised as being inside or outside of the burrow and those observed outside were further categorised by location: in the open in shade, in the open in sunlight; in the open in dappled light or in shelter. The trails left by cotton spools made it possible to establish whether a female was in a burrow or difficult to detect in the environment. Females were categorised as "in shelter" when outside of a burrow but largely or completely obscured from the view of the observer (e.g. among dense foliage, rock piles, tangled patches of long grass). In most cases the females classed as being "in shelter" had their locations confirmed by the presence of cotton thread leading into and not emerging from the shelter. Each shelter was visually inspected when a tuatara was not present to confirm the location as a shelter and not a concealed burrow. If a female was seen to have moved around in the environment and returned to a burrow the excess thread was gathered and the spool re-tied to prevent possible entanglement. This was only done when animals were inside burrows to prevent the observer being seen and minimise possible disturbance to the tuatara.

Based on previous findings that tuatara take greater advantage of basking opportunities when opportunities are low (Besson \& Cree 2010) I also wanted to assess if there were any differences among populations in exploitation of the thermal environment. As I found no significant effect of time of day on diurnal emergence (see thermoregulatory behaviour results) I chose to assess this by comparing the percentage of observations categorised as "emerged" with air temperature for a single observational time point. I chose to compare all 11am observations (obtained over seven days of 
monitoring) among sites and did this for both the spring 2014 and autumn 2015 diurnal behaviour surveys.

I used a Kruskal-Wallis test to assess differences among sites in the range of diurnal temperatures recorded at observational time points $(1100,1300,1500$, and 1700$)$ for the spring 2014 study season. Post hoc pairwise comparisons between sites were then performed using Mann-Whitney $U$ tests. I investigated the impact of the fixed effects of air temperature and time of day on diurnal emergence (inside or outside of a burrow) of tuatara per study site using GLMMs with a binomial distribution and individual ID as a random effect. Models' relative fit to data were compared using $\mathrm{AIC}_{\mathrm{C}}$ values and the bestfit model compared to the null model using a likelihood-ratio test. I also ran separate GLMMs per survey season to assess differences in emergence by site using emergence as the outcome variable, study site as the fixed effect and individual ID as a random effect. Following analysis of inside versus outside of burrow behaviour, I narrowed our analyses to the behaviour of tuatara that were observed outside of burrows. As I found a very small percentage of observations to be of emerged tuatara in direct sunlight or dappled light (see results) I categorised above-ground tuatara as either "in the open" (including animals in sun, shade and dappled light) or "in shelter" for analysis of out-of-burrow behaviour. I followed the same statistical procedure detailed for assessing emergence behaviour to determine the effect of study site, air temperature and time of day on out-of-burrow location.

As $100 \%$ of the emerged tuatara observed at ZEALANDIA in autumn 2015 were found to be in the open when out of burrows (see Results), a GLMM could not be run on the data due to separation in the model, an instance in which one or more fixed effects perfectly predicts the binary outcome of interest. A common solution for dealing with separation has been to omit the fixed effect from the model entirely; however, this approach ignores evidently influential factors (Zorn 2005). An alternative approach suggests modification of the data through supplementation of observations with artificial data, on which the model is then run (Clogg et al. 1991). This approach has been criticised as inferior to the alternatives, but offers a simple solution that, in this case, required minimal interference with the original data (Zorn 2005). I supplemented our data by adding a single observation to the ZEALANDIA out-of-burrow observations, in which a single (fabricated) individual was observed in a shelter. This had minimal influence on the 
overall ZEALANDIA data, reducing the proportion of "in the open" observations from $100 \%$ to $98.6 \%$ of the total observations.

\subsection{Results}

\subsubsection{Habitat thermal quality}

Based on the spring 2014 and autumn 2015 nocturnal and diurnal emergence data I established that emergence was generally lower or close to zero at air temperatures of $10^{\circ} \mathrm{C}$ and below for the females studied, so I selected this temperature as the lower temperature threshold for emergence degree day calculations. As the preferred body temperature of adult male and unsexed juvenile tuatara has been recorded as ranging between $19.5^{\circ} \mathrm{C}$ and $23.1^{\circ} \mathrm{C}$ (mean $=21.3^{\circ} \mathrm{C}$; Besson and Cree 2010), I used $19^{\circ} \mathrm{C}$ as the baseline temperature for assessing the number of preferred temperature degree days per site. It is important to note here that the preferred temperature of adult female tuatara is unknown, but I have used the data from adult males and juveniles to provide an estimate. Based on the spring 2014 diurnal emergence data for Cape Sanctuary (the only site to experience air temperatures of $25^{\circ} \mathrm{C}$ and above during observations) I established that emergence declined at air temperatures approaching and above $25^{\circ} \mathrm{C}$, so selected this temperature as the upper temperature threshold for emergence degree day calculations.

I found that three of the four October 2012 translocation sites (Cape Sanctuary, Young Nicks Head and Whangaokeno) experienced higher numbers of $>10^{\circ} \mathrm{C}$ degree days per month than the founder or control site, with some sites (e.g. Whangaokeno) experiencing four to six times the number of total degree days calculated for Stephens Island in the colder winter months (e.g. July and August), indicating warmer climates yearround at these three translocation sites (Figure 5.3). Conversely, the mountainous Maungatautari site was climatically similar to the founder and control sites for most of the year, but showed a comparatively higher number of degree days over the summer months (December-February, Figure 5.3). I found a similar pattern in the total number of $>19^{\circ} \mathrm{C}$ degree days per site, with the Cape Sanctuary, Young Nicks Head and Whangaokeno sites experiencing higher numbers of $>19^{\circ} \mathrm{C}$ degree days than the founder 
or control site, especially over the summer months, when the number of degree days at these sites were two to eighteen times higher than those calculated for Stephens Island and ZEALANDIA. As with emergence degree days, Maungatautari was comparable to Stephens Island and ZEALANDIA for much of the year, but demonstrated a higher number of $>19^{\circ} \mathrm{C}$ degree days over summer (Figure 5.3). I also found that the three warmer sites, Cape Sanctuary, Young Nicks Head and Whangaokeno, experienced more $>25^{\circ} \mathrm{C}$ degree days than the founder, control or Maungatautari sites, with Stephens Island experiencing zero $>25^{\circ} \mathrm{C}$ days throughout the year and Maungatautari and ZEALANDIA experiencing one and four $>25^{\circ} \mathrm{C}$ degree days (respectively) in January, the warmest month. It was also interesting to note that, among the warmer sites, Whangaokeno experienced consistently lower numbers of $>25^{\circ} \mathrm{C}$ degree days than Young Nicks Head or Cape Sanctuary, whereas in January Cape Sanctuary experienced over double the number of $>25^{\circ} \mathrm{C}$ degree days seen at any other site and almost double the number in February. As the sum of degree day values is representative of both the number of days that a baseline temperature was exceeded as well as the degree to which this temperature was exceeded, this indicates that, of the sites studied, Cape Sanctuary experienced more instances in late spring and summer (November - February) in which temperatures exceeded the upper limit for emergence from burrows. It is important to acknowledge here, however, that the air temperature data used in these calculations were obtained from one HOBO datalogger per site and so the comparisons made between sites are qualitative.

The iButton dataloggers that were attached to adult female tuatara at four of the study sites were used to assess the range of skin temperatures experienced in May 2014 at these sites. Due to iButton drop off over time I was unable to obtain skin temperature data from all females to which iButtons were originally attached, therefore sample sizes from which mean skin temperature data were obtained for May 2014 were small (Cape Sanctuary = three; Maungatautari $=$ six; ZEALANDIA = four; Stephens Island $=$ two). The monthly air temperature range per site (obtained from the HOBO datalogger placed at each site) for May 2014 was as follows: Cape Sanctuary: $5-25^{\circ} \mathrm{C}$; Maungatautari: $0-16^{\circ} \mathrm{C}$; ZEALANDIA: $2-17^{\circ} \mathrm{C}$; Stephens Island: $6-17^{\circ} \mathrm{C}$. I found that the range of minimum mean daily skin temperatures $(H(3)=32.743, p<0.001)$, maximum mean daily skin temperatures $(H(3)=27.023, p<0.001)$ and mean daily skin temperatures $(H(3)=39.027$, $p<0.001)$ differed significantly by site, with a significantly higher range of maximum skin 
temperatures recorded in Cape Sanctuary females when compared to other sites (Maungatautari: $W=790, p<0.001 ;$ ZEALANDIA: $W=698.5, p=0.002$; Stephens Island: $W=770.5, p<0.001$ ), and only these females were able to obtain skin temperatures within the tuatara's estimated preferred body temperature range in May 2014 (Figure 5.4). ZEALANDIA females also attained higher maximum temperatures than Maungatautari females $(\mathrm{W}=306.5, \mathrm{p}=0.015)$, but there were no differences in maximum skin temperature ranges observed between Stephens Island and Maungatautari $(W=445$, $p=0.622$ ) or ZEALANDIA ( $\mathrm{W}=598, \mathrm{p}=0.099)$. Regarding the minimum and mean skin temperatures obtained per site, the Stephens Island females attained the lowest range of minimum skin temperatures of all four study sites (Cape Sanctuary: $W=719, p<0.001$; Maungatautari: $W=756.5, p<0.001$; ZEALANDIA: $W=844, p<0.001$ ), as well as the lowest range of mean skin temperatures (Cape Sanctuary: $W=804.5, p<0.001$; Maungatautari: $W=739.5, p<0.001$; ZEALANDIA: $W=818, p<0.001$ ). Females at ZEALANDIA also experienced a significantly higher range of minimum skin temperatures than females at Cape Sanctuary $(W=303, p=0.013)$ and Maungatautari $(W=299, p=$ 0.011), and both Cape Sanctuary and ZEALANDIA females experienced warmer mean skin temperatures than Maungatautari females (Cape Sanctuary: $W=713.5, p=0.001$; ZEALANDIA: $\mathrm{W}=232.5, \mathrm{p}<0.001)$. Tuatara skin temperature correlated significantly with air temperature at all four study sites (Cape Sanctuary: $r_{s}=0.91, p<0.001$; Maungatautari: $r_{\mathrm{s}}=0.56, p<0.001 ;$ ZEALANDIA: $r_{\mathrm{s}}=0.87, \mathrm{p}<0.001$; Stephens Island: $r_{\mathrm{s}}=0.92, \mathrm{p}<0.001$, Figure 5.4).

It is also apparent from Figure 5.4 that the correlation between female tuatara body and air temperatures at Maungatautari and ZEALANDIA followed the isothermal line (a representation of points at which air and body temperatures are the same) reasonably closely at air temperatures above $\sim 10^{\circ} \mathrm{C}$, but deviated at lower air temperatures, indicating that females were able to maintain warmer body than air temperatures. Similarly, Cape Sanctuary females show warmer body than air temperatures at lower air temperatures $\left(\sim 10^{\circ} \mathrm{C}\right)$, but body temperatures are shown to be cooler at higher air temperatures approaching $20^{\circ} \mathrm{C}$. Conversely, the Stephens Island females follow reasonably closely to the isothermal line, but generally maintain slightly cooler body than air temperatures. 
To investigate the range of temperatures available to tuatara in burrows I compared the monthly minimum and maximum burrow temperatures and air temperatures per site from May 2014 to February 2015 (when burrow and air temperature data were available for all four sites). I found that the range of burrow temperatures experienced fell consistently within the range of air temperatures recorded, demonstrating higher minimum temperatures and lower maximum temperatures in every month (Figure 5.5). Burrow maximum and minimum temperatures demonstrated similar patterns of monthly variation seen in air temperature, suggesting that burrow temperatures fluctuate with air temperature. Maximum burrow temperatures ranged from $11-23^{\circ} \mathrm{C}$ and were higher at Cape Sanctuary than other sites. Minimum burrow temperatures ranged from $5-16^{\circ} \mathrm{C}$ and were generally lower at Maungatautari and ZEALANDIA, with the exception of January and February when minimum burrow temperatures at Maungatautari were similar to those observed at Cape Sanctuary, likely due to the warmer minimum air temperatures experienced at Maungatautari over summer months. Minimum burrow temperatures were between one and seven degrees warmer than minimum air temperatures, with the greatest burrow-air temperature differences observed at Maungatautari and the smallest differences observed at Cape Sanctuary. Maximum burrow temperatures were between zero and twelve degrees lower than air temperatures, with the smallest differences observed on Stephens Island and the largest differences observed at Cape Sanctuary. 

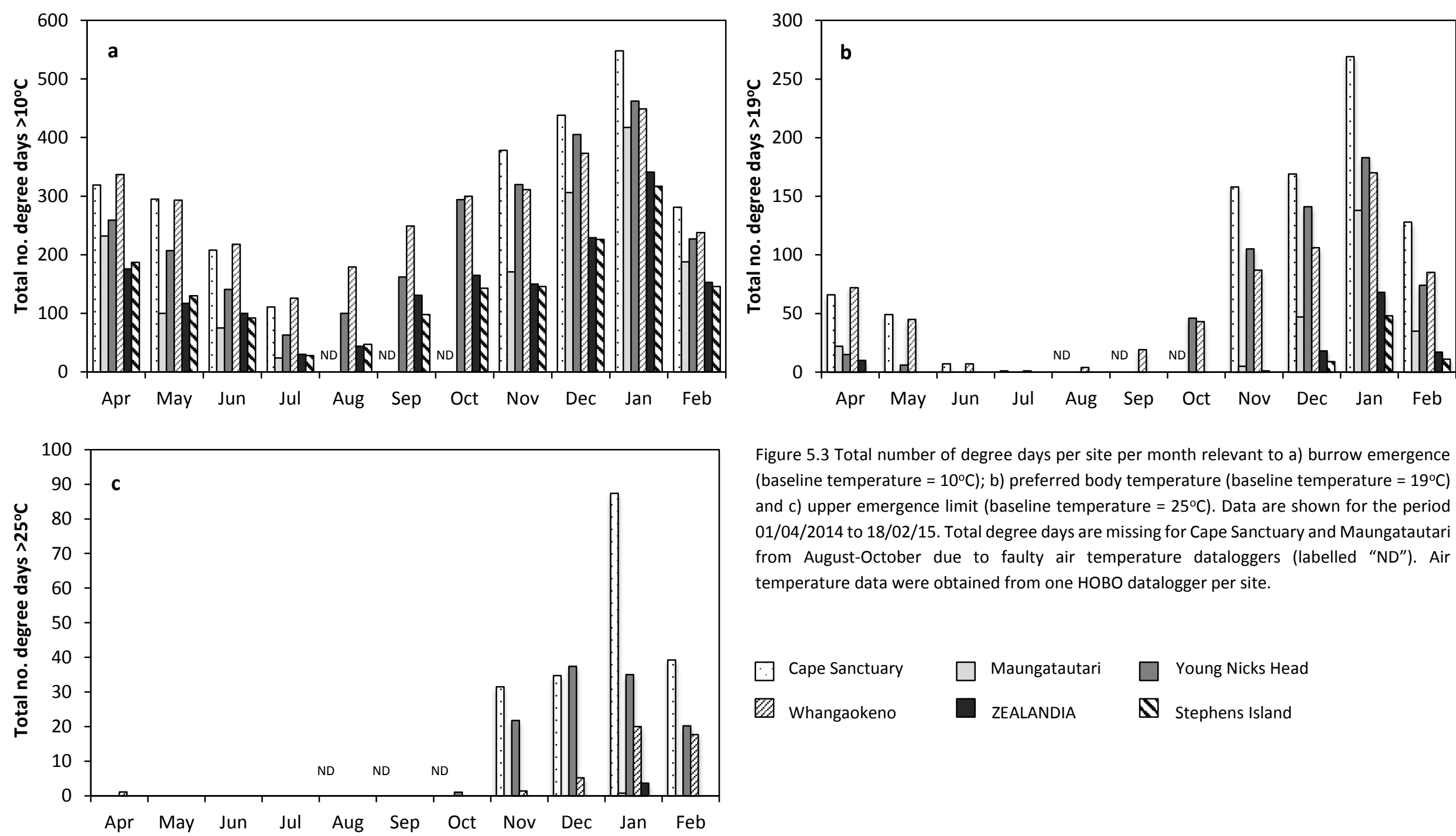

Figure 5.3 Total number of degree days per site per month relevant to a) burrow emergence (baseline temperature $=10^{\circ} \mathrm{C}$ ); b) preferred body temperature (baseline temperature $=19^{\circ} \mathrm{C}$ ) and c) upper emergence limit (baseline temperature $=25^{\circ} \mathrm{C}$ ). Data are shown for the period 01/04/2014 to 18/02/15. Total degree days are missing for Cape Sanctuary and Maungatautari from August-October due to faulty air temperature dataloggers (labelled "ND"). Air temperature data were obtained from one $\mathrm{HOBO}$ datalogger per site.

$\begin{array}{ll}\square \text { Cape Sanctuary } & \square \text { Maungatautari } \\ \square \text { Whangaokeno } & \square \text { ZeuLANDIA }\end{array}$ 

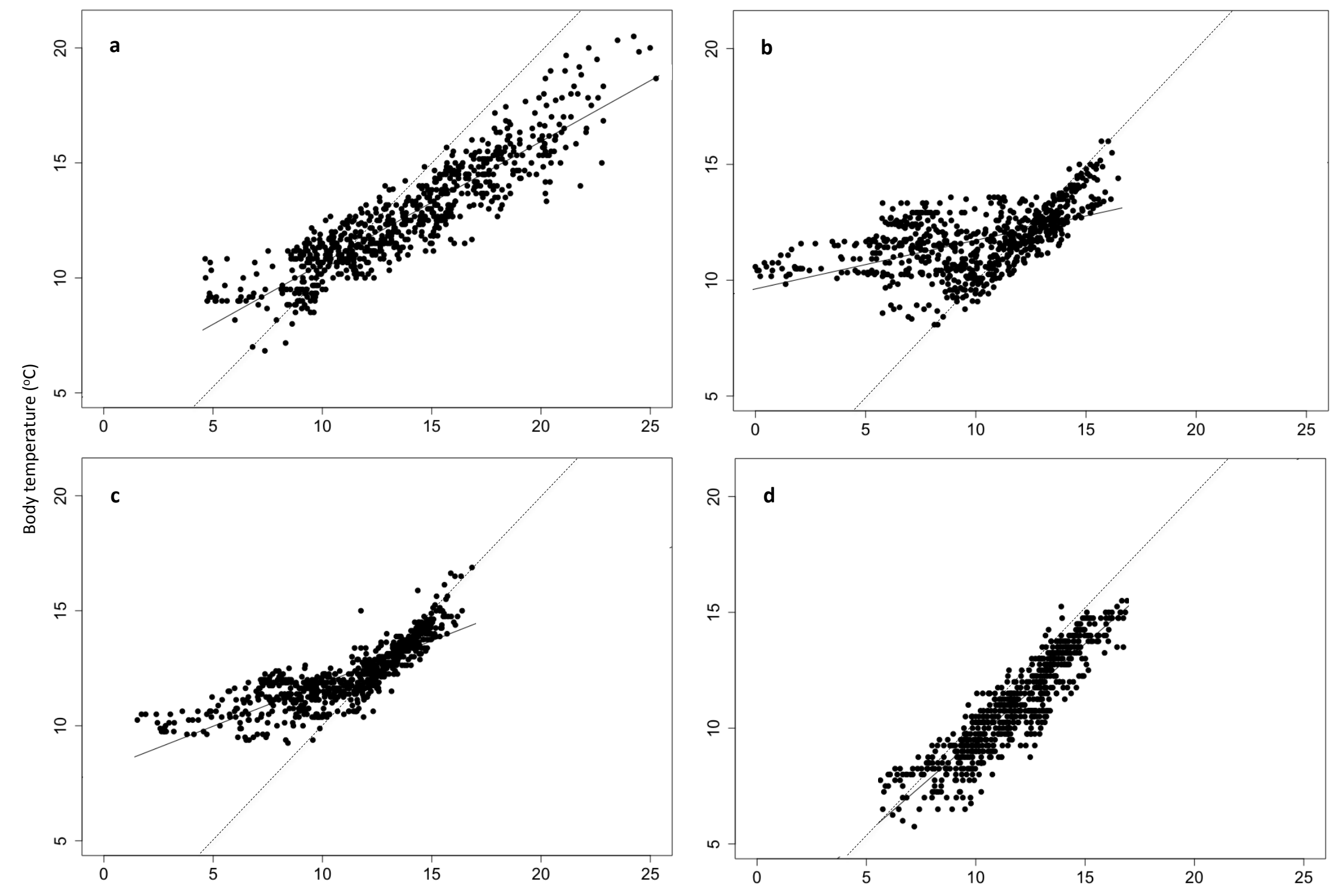

Figure 5.4 Scatterplots of correlations between hourly air temperatures and mean hourly tuatara body temperatures for May 2014. Graph $a=C a p e$ Sanctuary $(n=3), b=$ Maungatautari $(n=6), c=$ ZEALANDIA $(n=4), d=$ Stephens Island $(n=2)$. Different sample sizes are due to iButton drop off during study. Line of best fit (solid line) and isothermal line (dashed line) shown. 

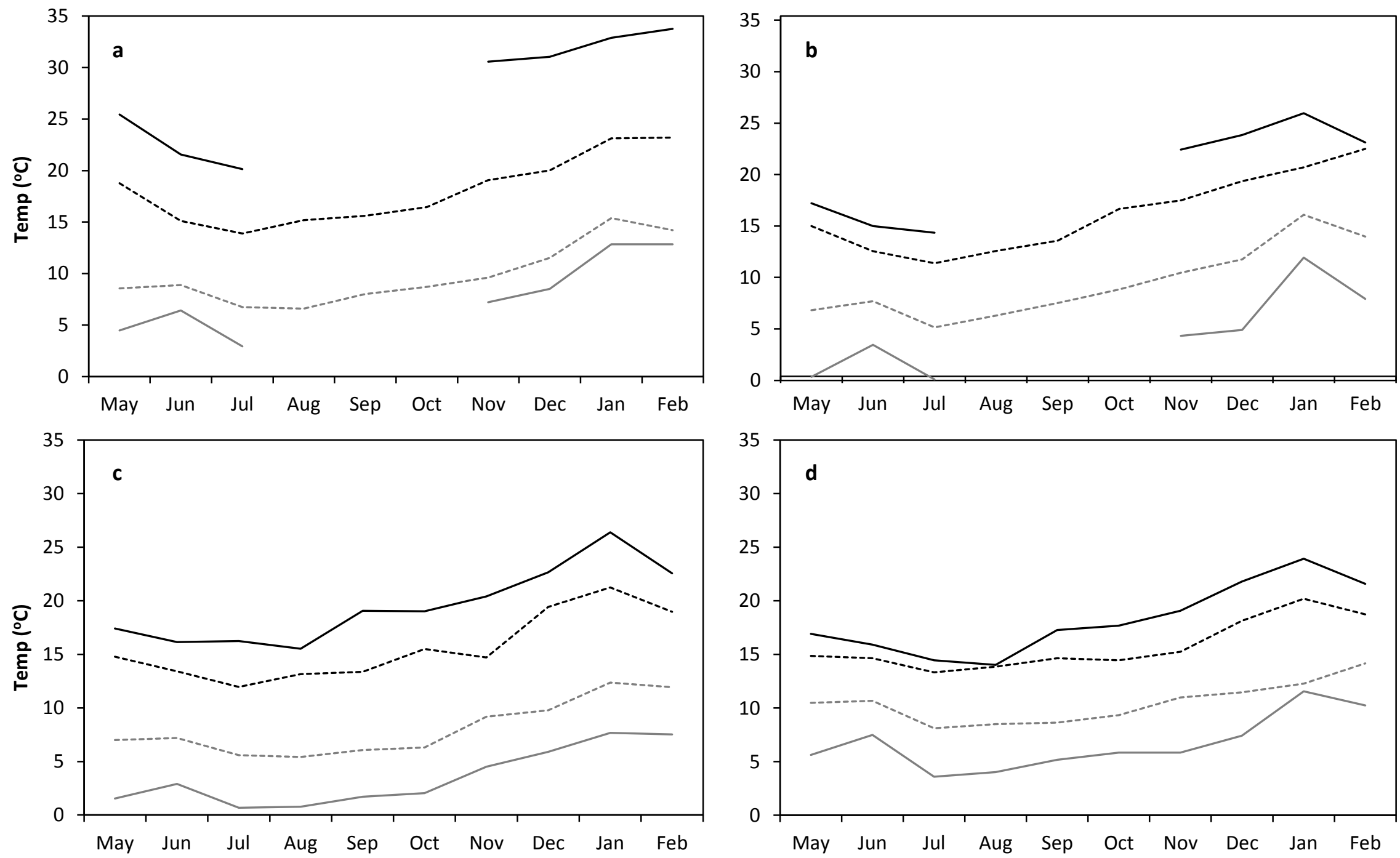

Figure 5.5 Graphs of minimum and maximum monthly burrow and air temperatures per site from May 2014 to February 2015. Graph a = Cape Sanctuary, b = Maungatautari, c = ZEALANDIA, $d=$ Stephens Island. Air temperature data for Cape Sanctuary and Maungatautari from August-October are missing due to faulty air temperature dataloggers.

Maximum air temp CMinimum air temp. - - Maximum burrow temp. - 


\subsubsection{Nocturnal activity}

Due to a combination of low initial captures at some sites and the loss of some spools (owing to wet conditions, moulting or the thread snapping), between 2 and 10 female tuatara were monitored per site per night in spring 2014. Distances moved overnight were highly variable and ranged between 0.30-73.56 $\mathrm{m}$ in spring 2014 and 0.45-70.16 m in autumn 2015. Displacement was similarly variable, with tuatara moving $0.15-70 \mathrm{~m}$ per night away from burrows in spring 2014 and 0.2-22 m per night in autumn 2015. Between 38\% and 76\% of spring 2014 and autumn 2015 total observations saw individuals leave a location and move to a new one overnight (spring 2014: Cape Sanctuary: 50\%; Maungatautari: 40\%; ZEALANDIA: 47\%; autumn 2015: Cape Sanctuary: 57\%; Maungatautari: 38\%; Stephens Island: 39\%; ZEALANDIA: 76\%) while the remainder saw individuals emerge and return to the same burrow/shelter. Tuatara were generally observed to occupy 1-2 burrows and several shelters per site, though some individuals at Maungatautari and Stephens Island occupied up to three burrows, some ZEALANDIA individuals occupied up to four burrows, and, at most, one Cape Sanctuary individual was seen to move between five different burrows.

Mean nocturnal temperatures recorded during spring 2014 observations ranged between $7.76^{\circ} \mathrm{C}$ and $19.15^{\circ} \mathrm{C}$ and tuatara were observed outside of burrows across all mean temperatures. The range of mean overnight temperatures recorded differed significantly by site $(\mathrm{H}(2)=11.682, \mathrm{p}=0.003)$, with Maungatautari and ZEALANDIA experiencing significantly cooler nocturnal climates than Cape Sanctuary (Maungatautari: $\mathrm{W}=36, \mathrm{p}=0.002$; ZEALANDIA: $\mathrm{W}=35.5, \mathrm{p}=0.006$ ), while Maungatautari and ZEALANDIA did not differ significantly ( $W=25, p=0.310$; Figure 5.6). I found significant effects of mean overnight air temperature on log-transformed net distance moved $(\chi 2(1)=8.73, \chi 2$ prob. $=0.003)$ and log-transformed displacement $(\chi 2(1)=9.649, \chi 2$ prob. $=0.002)$ in spring 2014, with distance and displacement both found to increase with air temperature (Figure 5.7). The addition of population density did not improve the models for either distance $(\chi 2(1)=0.15, \chi 2$ prob. $=0.698)$ or displacement $(\chi 2(1)=1.427, \chi 2$ prob. $=0.232)$, indicating no significant effect of population density on nocturnal activity. There were no significant differences among sites in net distance $(H(2)=0.756, p=0.685)$ or displacement $(H(2)=$ $0.703, p=0.704$ ) moved overnight. Regarding emergence behaviour, the model including 
overnight temperature was a significant improvement over the null $(\chi 2(1)=6.509, \chi 2$ prob. $=0.012)$ and demonstrated a significant effect of temperature on whether an individual emerged from the burrow (estimate $=0.214 \pm 0.091, z=2.361, p=0.018$; Figure 5.7), whereas the model including site as a fixed effect was not significant $(\chi 2(2)=2.522$, $\chi 2$ prob. $=0.283)$, indicating no significant differences in tuatara emergence among the spring 2014 study sites.

Mean nocturnal temperatures recorded during autumn 2015 monitoring ranged between $7.73^{\circ} \mathrm{C}$ and $16.42^{\circ} \mathrm{C}$ and tuatara were observed outside of burrows across all mean temperatures. The range of mean overnight temperatures differed significantly by site $(H(3)=32.377, p<0.001)$, with Cape Sanctuary experiencing a significantly lower range than Maungatautari and Stephens Island (Maungatautari: $W=31, p=0.041$; Stephens Island: $W=5, p=0.041)$, but the mean temperature ranges did not differ significantly among the other sites ( $p>0.05$; Figure 5.6). Log-transformed net distance was significantly influenced by overnight temperature in autumn $2015(\chi 2(1)=9.386, \chi 2$ prob. $=0.002$; Figure 5.8), and the addition of population density did not improve the model $(\chi 2(1)=0.261, \chi 2$ prob. $=0.610)$, demonstrating no significant influence of density on net distance moved. I found no significant effect of overnight temperature $(\chi 2(1)=$ $0.319, \chi 2$ prob. $=0.572)$ or population density $(\chi 2(1)=0.3812, \chi 2$ prob. $=0.537)$ on logtransformed displacement. There were no significant differences among study sites in overnight displacement $(H(3)=5.853, p=0.119)$, but a significant effect of site on net distance moved $(H(3)=28.694, p<0.001)$. Maungatautari females moved significantly further than females at all other study sites and Stephens Island females moved significantly further than Cape Sanctuary females. The distances moved by ZEALANDIA females were not significantly different to those recorded at Cape Sanctuary or Stephens Island (Table 5.2, Figure 5.9). Regarding emergence behaviour, I found that the model including overnight temperature as a fixed effect was the best fit (Table 5.3) and showed that air temperature significantly influenced nocturnal emergence of tuatara $(\chi 2(1)=$ $39.279, \chi 2$ prob. $<0.001$, estimate $=0.534 \pm 0.102, z=5.266, p<0.001)$, with greater proportions of the observed females shown to leave their burrows on nights when air temperatures were warmer. 


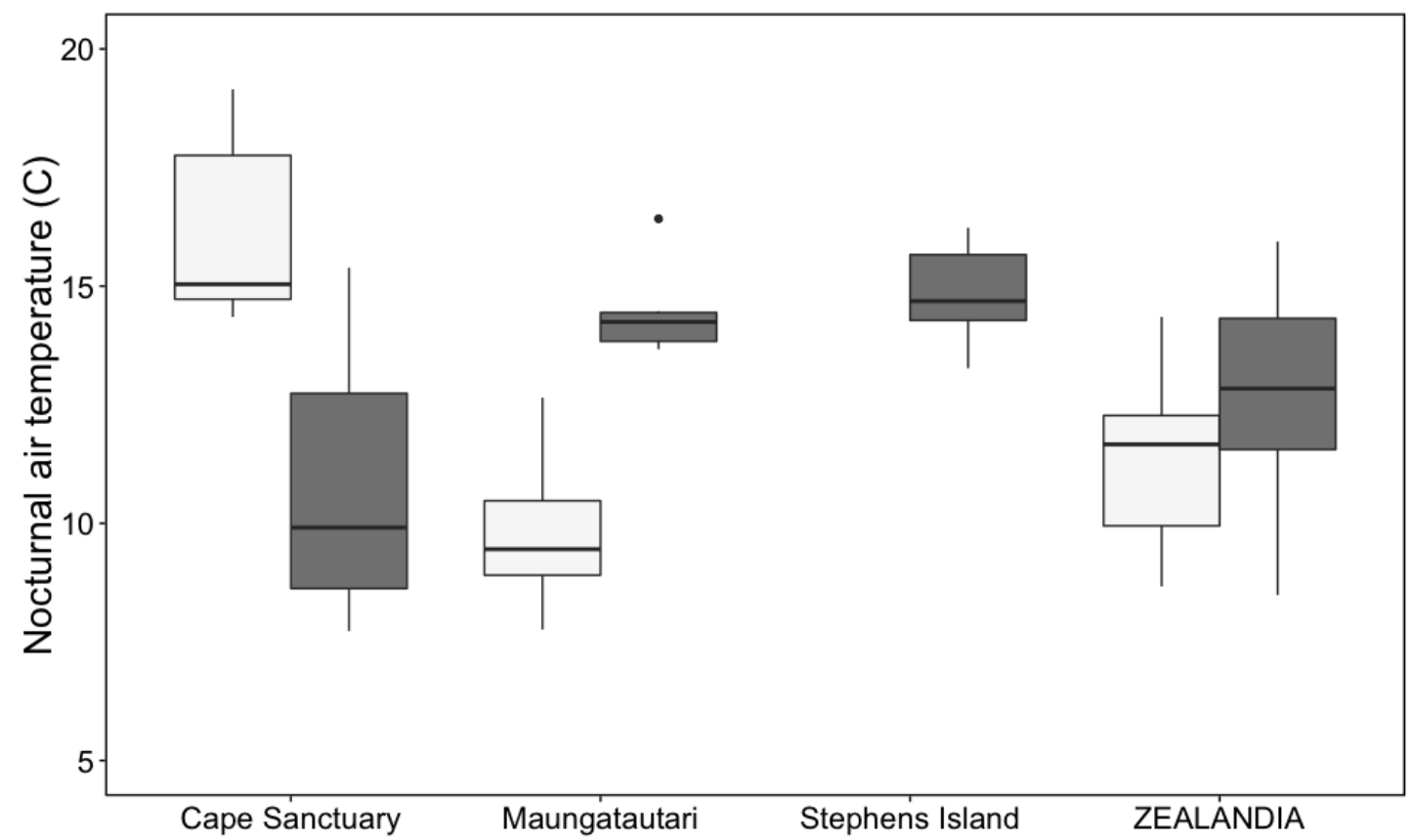

Figure 5.6 Boxplots showing range of mean overnight temperatures recorded over six nights per site for the spring 2014 (pale grey) and autumn 2015 (dark grey) nocturnal activity surveys. Boxplots show the median and interquartile range and whiskers extend up to 1.5 times the interquartile range. No temperature data are shown for Stephens Island in spring 2014 as this site was not visited in that season. Black dot shows an outlier in the autumn 2015 data. Overnight temperatures were obtained from one HOBO datalogger per site. 

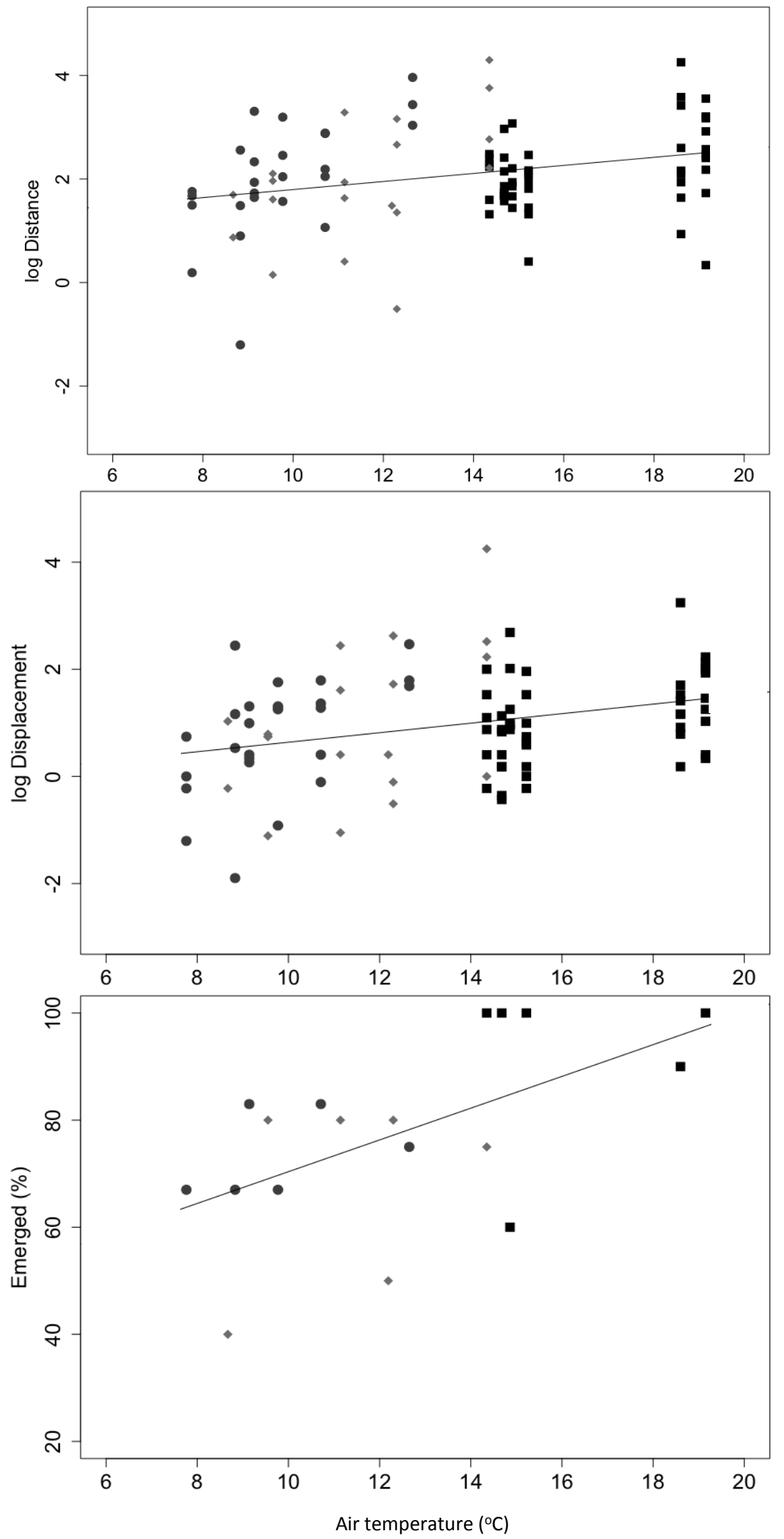

Figure 5.7 Spring 2014 log-transformed net distance and displacement by mean overnight air temperature. Emergence of tuatara shown as percentage of observed animals per site per night found to have left the burrow. Line shows the best fit line. $\mathbf{\square}=$ Cape Sanctuary, $\bullet=$ Maungatautari, $\checkmark=$ ZEALANDIA. 

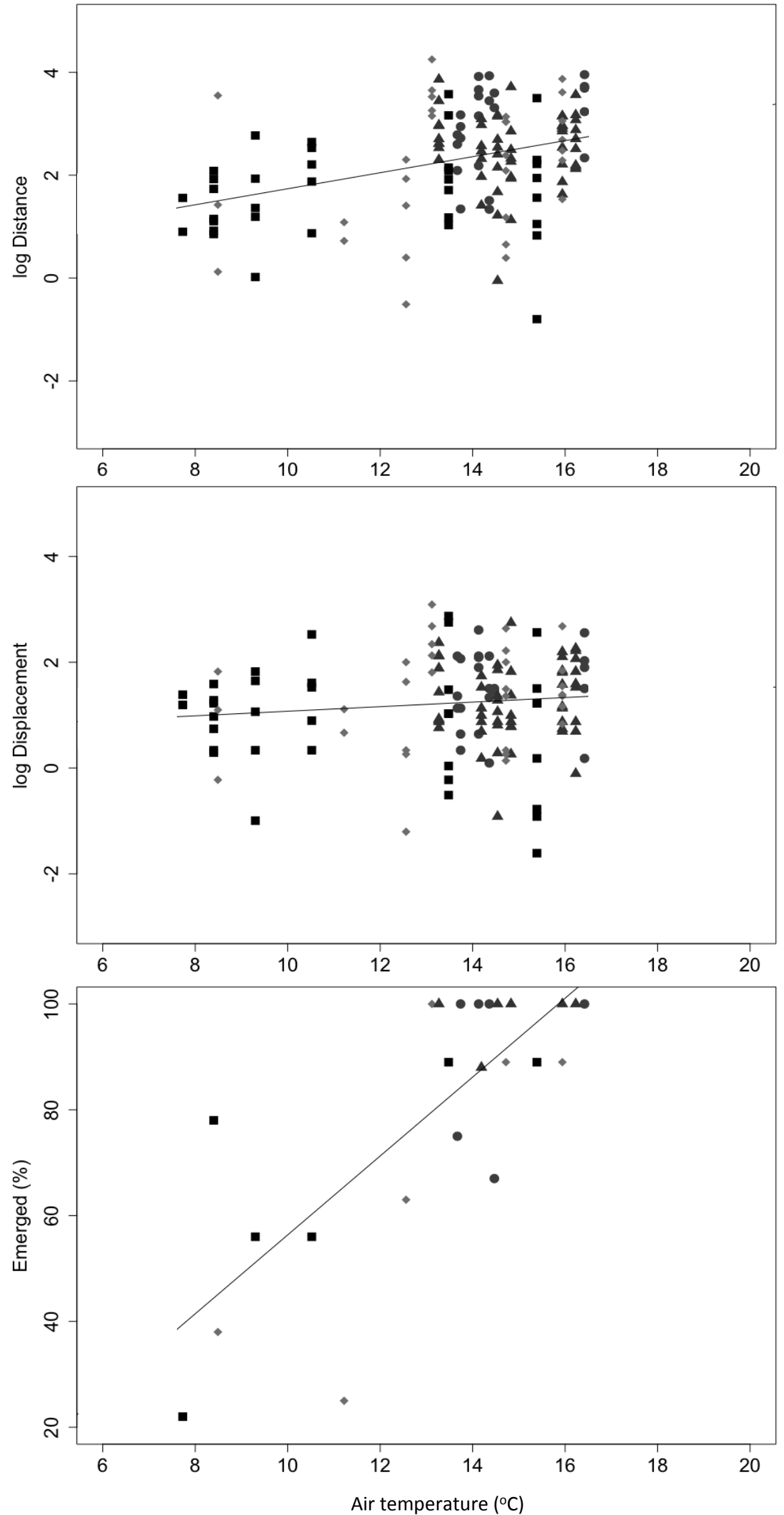

Figure 5.8 Autumn 2015 log-transformed net distance and displacement by mean overnight air temperature. Emergence of tuatara shown as percentage of observed animals per site per night found to have left the burrow. Line shows the best fit line. $\mathbf{\square}=$ Cape Sanctuary,

- = Maungatautari, $\diamond$ = ZEALANDIA, $\mathbf{\Delta}$ = Stephens Island. 
Table 5.2 Results of Mann-Whitney U tests. Pairwise comparisons were made of net distances moved at each site. I considered a $p$-value of $p<0.05$ to indicate statistical significance. Net distance data are shown in Figure 5.9.

\begin{tabular}{|l|ccccc|}
\cline { 2 - 6 } \multicolumn{1}{c|}{} & $\begin{array}{c}\text { Cape } \\
\text { Sanctuary }\end{array}$ & Maungatautari & $\begin{array}{c}\text { Stephens } \\
\text { Island }\end{array}$ & ZEALANDIA \\
\hline Cape Sanctuary & - & $\mathrm{W}=117$, & $\mathrm{W}=379$, & $\mathrm{W}=383.5$, \\
& & $\mathrm{p}<0.001$ & $\mathrm{p}<0.001$ & $\mathrm{p}=0.064$ \\
Maungatautari & - & - & $\mathrm{W}=372.5$, & $\mathrm{W}=213.5$, \\
& & & $\mathrm{p}=0.010$ & $\mathrm{p}=0.019$ \\
Stephens Island & - & - & - & $\mathrm{W}=860.5$, \\
& & & & & \\
\end{tabular}

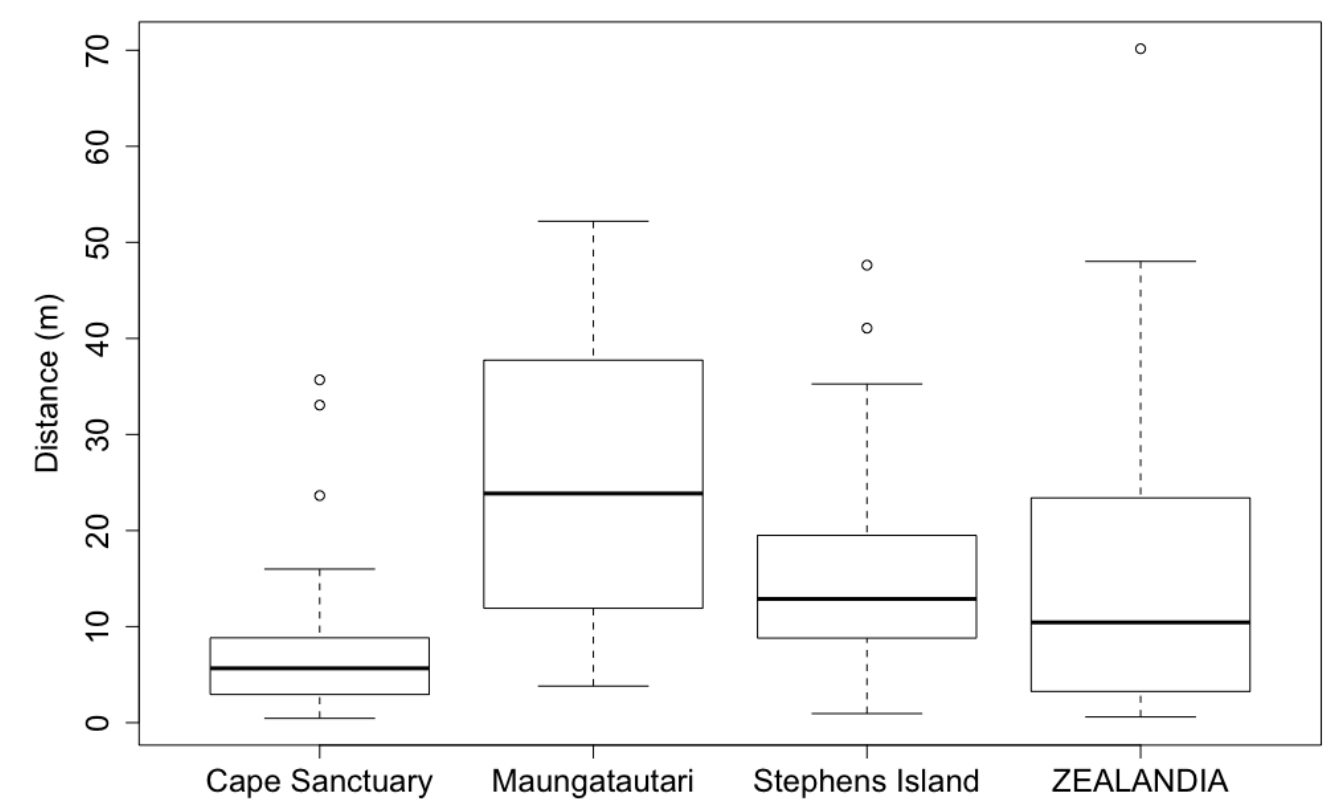

Figure 5.9 Boxplots of net distance moved over six nights of nocturnal monitoring by study site. Boxplots show the median and interquartile range and whiskers extend up to 1.5 times the interquartile range. Dots show outliers in the data.

Table 5.3 Results of analysis of competing GLMMs on factors associated with nocturnal emergence in the autumn 2015 survey season. $\mathrm{K}=$ number of estimated parameters in the model. $\mathrm{AlC}_{\mathrm{C}}=$ second-order Akaike information criterion. $\Delta\left(A I C_{C}\right)=\left[A I C_{C}-\min \left(A I C_{C}\right)\right]$. Relative likelihood $=\exp \left(-0.5^{*} \Delta\left(A I C_{C}\right)\right) . w\left(A I C_{C}\right)=$ weight of the model $=$ relative likelihood/sum of likelihoods across both models.

\begin{tabular}{lrcrrr}
\hline \multicolumn{1}{c}{ Model fixed effects } & K & AIC & $\Delta\left(A \mid C_{c}\right)$ & $\begin{array}{c}\text { Relative } \\
\text { likelihood }\end{array}$ & w(AIC $)$ \\
\hline Air temperature & 3 & 143.260 & 0 & 1 & 1 \\
Site & 5 & 160.626 & 17.366 & 0.000 & 0.000 \\
Null model & 1 & 180.406 & - & - & - \\
\hline
\end{tabular}




\subsubsection{Thermoregulatory behaviour}

The range of diurnal air temperatures recorded at observational time points $(1100,1300$, 1500 and 1700 ) during the spring 2014 monitoring ranged between $10.15^{\circ} \mathrm{C}$ and $25.70^{\circ} \mathrm{C}$ and differed significantly by site $(H(2)=48.540, p<0.001$, Figure 5.10$)$. Maungatautari experienced a significantly warmer range of diurnal air temperatures than ZEALANDIA (W $=212.5, p=0.003)$, and Cape Sanctuary was significantly warmer than both Maungatautari and ZEALANDIA (Maungatautari: $W=717, p<0.001$; ZEALANDIA: $W=763$, $p<0.001$, Figure 5.10). I found that the best-fit models showed significant effects of air temperature on daytime emergence for both the Cape Sanctuary and ZEALANDIA populations (Table 5.4, Figure 5.11), but no significant effect of air temperature on emergence in the Maungatautari population $(\chi 2(3)=5.150, \chi 2$ prob. $=0.161)$, and I found no influence of time of day on burrow emergence in any of the populations studied. The ZEALANDIA emergence data showed a positive relationship with air temperature, with emergence increasing to $100 \%$ at $15-16^{\circ} \mathrm{C}$, whereas emergence at Cape Sanctuary was relatively stable between $14^{\circ} \mathrm{C}$ and $23^{\circ} \mathrm{C}$, but showed clear declines at temperatures approaching and above $25^{\circ} \mathrm{C}$ (Figure 5.11).

The range of diurnal temperatures recorded at observational time points in autumn 2015 ranged between $8.9^{\circ} \mathrm{C}$ and $22.3^{\circ} \mathrm{C}$ and also differed significantly by site $\left(\mathrm{H}_{(3)}\right.$ $=35.589, \mathrm{p}<0.001$, Figure 5.10), with Maungatautari experiencing a significantly warmer range of observational temperatures than all other study sites (Cape Sanctuary: $W=149$, $p<0.001$; Stephens Island: $W=610.5, p<0.001$; ZEALANDIA: $W=56, p<0.001$ ) and Stephens Island found to be significantly warmer than ZEALANDIA ( $W=213.5, p=0.004)$. Observational temperatures did not differ significantly between Cape Sanctuary and Stephens Island ( $W=301, p=0.138$ ) or ZEALANDIA ( $W=463.5, p=0.245)$. I assessed factors influencing diurnal emergence at each study site and found significant effects of air temperature on the emergence of female tuatara at all four study sites, with the proportion of emerged females seen to increase positively with air temperature (Table 5.4, Figure 5.12). Time of day was not found to significantly influence emergence.

I found no significant effect of study site on overall emergence over the seven days of behavioural observations in spring $2014\left(\chi 2_{(2)}=1.700, \chi 2\right.$ prob. $\left.=0.427\right)$ or autumn 2015 $\left(\chi 2_{(3)}=1.016, \chi 2\right.$ prob. $\left.=0.798\right)$, indicating that, despite significant differences in diurnal 
observational temperature ranges and significant associations between air temperature and emergence at most study sites, this did not result in significantly overall greater or reduced emergence at any of the sites.

I found a very small percentage of observations to be of emerged tuatara in direct sunlight or dappled light (spring 2014: sunlight $=0.72 \%$, dappled light $=2.15 \%$; autumn 2015: sunlight $=0 \%$, dappled light $=6.27 \%$ ) so observations were re-categorised as "in the open" (incorporating animals observed in sun, shade and dappled light) or "in shelter" for analysis of out-of-burrow behaviour. In the majority of total observations per site emerged tuatara concealed themselves in shelter rather than positioning themselves in the open, with the exception of the autumn 2015 ZEALANDIA observations, in which $100 \%$ of out-of-burrow observations were of tuatara in the open (Figure 5.13). Although a larger proportion of the total out-of-burrow observations at Maungatautari showed tuatara to occupy shelters rather than open ground in spring 2014, there were no significant differences in out-of-burrow behaviour among study sites $\left(\chi 2_{(2)}=3.991, \chi 2\right.$ prob. $\left.=0.136\right)$. However, behaviour did differ by site during the autumn 2015 observations $\left(x 2_{(3)}=\right.$ 15.233, $\chi 2$ prob. $=0.002)$, with a greater proportion of observations at ZEALANDIA showing females in the open when compared to the other study sites (Cape Sanctuary: estimate $=-10.685 \pm 3.387, z=-3.154, p=0.002 ;$ Maungatautari: estimate $=-8.538 \pm$ 3.016, $z=-2.831, p=0.005 ;$ Stephens Island: estimate $=-6.712 \pm 2.894, z=-2.320, p=$ 0.020, Figure 5.13).

I found significant effects of air temperature on whether tuatara were observed in shelter or in the open for the Cape Sanctuary spring $2014\left(\chi 2_{(1)}=10.252, \chi 2\right.$ prob. $=0.001$, estimate $=0.267 \pm 0.089, z=3.004, p=0.003)$ and Maungatautari autumn 2015 surveys $\left(\chi 2_{(1)}=5.272, \chi 2\right.$ prob. $=0.022$, estimate $\left.=0.429 \pm 0.198, z=2.160, p=0.031\right)$, with greater proportions of emerged individuals observed in shelter at lower air temperatures (Figure 5.14). No effect of temperature or time of day was found in any of the other surveys as models were either not significant explanations of the data or found no significant influence of the fixed effect (spring 2014: Maungatautari: $\chi 2_{(1)}=1.253, \chi 2$ prob. $=0.263$; ZEALANDIA: $\chi 2_{(1)}=2.430, \chi 2$ prob. $=0.119$; autumn 2015: Cape Sanctuary: $\chi 2_{(1)}=9.322$, $\chi 2$ prob. $=0.002$, estimate $=0.906 \pm 0.584, z=1.552, p=0.121 ;$ Stephens Island: $\chi 2_{(1)}=$ $0.152, \chi 2$ prob. $=0.697)$. As the emerged tuatara at ZEALANDIA exhibited a constant response (i.e. were exclusively observed in the open when outside of burrows) I was 
unable to assess the relationship between air temperature and location, however it is evident that ZEALANDIA females emerged into open spaces regardless of the variation in air temperature, which ranged from $12.26-18.25^{\circ} \mathrm{C}$.

Following comparisons of emergence and temperature at 1100 among sites I found that, despite Cape Sanctuary experiencing a substantially higher range of air temperature at 1100 in spring $2014\left(14.4-25.7^{\circ} \mathrm{C}\right)$, the percentage of individuals that were emerged when air temperatures were within or above the tuatara's preferred body temperature range $\left(19.5-23.1^{\circ} \mathrm{C}\right)$ was comparable to emergence seen over seven days of observations at the cooler Maungatautari and ZEALANDIA sites (Cape Sanctuary: 20-70\%; Maungatautari: 17-67\%; ZEALANDIA: 17-60\%; see Appendix 5, Table A5.1). This suggests that many of the Cape Sanctuary females did not emerge from burrows and take advantage of opportunities to obtain skin temperatures within their preferred range when air temperatures allowed. However, as none of the other spring 2014 study sites experienced temperatures within the preferred range I am unable to say if individuals in these populations would have taken advantage of such thermal opportunities and demonstrated higher emergence. To determine why some females may have remained in burrows at presumably beneficial air temperatures I examined the mean burrow temperatures recorded at the 1100 observations. I found burrow temperatures to range between $13.4^{\circ} \mathrm{C}$ and $18.0^{\circ} \mathrm{C}$, which suggests that female tuatara were not selecting burrows because they were able to achieve skin temperatures within their preferred range without having to emerge, but suggests that emergence was either restricted for some females or that, owing to the warmer overall climate experienced at Cape Sanctuary during this monitoring period (Figure 5.10), females were not thermoregulating as strictly due to the increased thermal opportunities at the site at that time, meaning that they did not need to emerge as often. There was only one instance in which air temperature at 1100 exceeded $19.5^{\circ} \mathrm{C}$ in autumn 2015 so I was unable to examine emergence behaviour over a range of suitable air temperatures. However, on this single occasion air temperature was recorded as $19.7^{\circ} \mathrm{C}$ at Maungatautari, the warmest site during the autumn 2015 survey (Figure 5.10), and emergence was zero per cent, which could also be taken as an indicator that female tuatara at this site were not thermoregulating as strictly owing to greater thermal opportunities at that site in that period. 


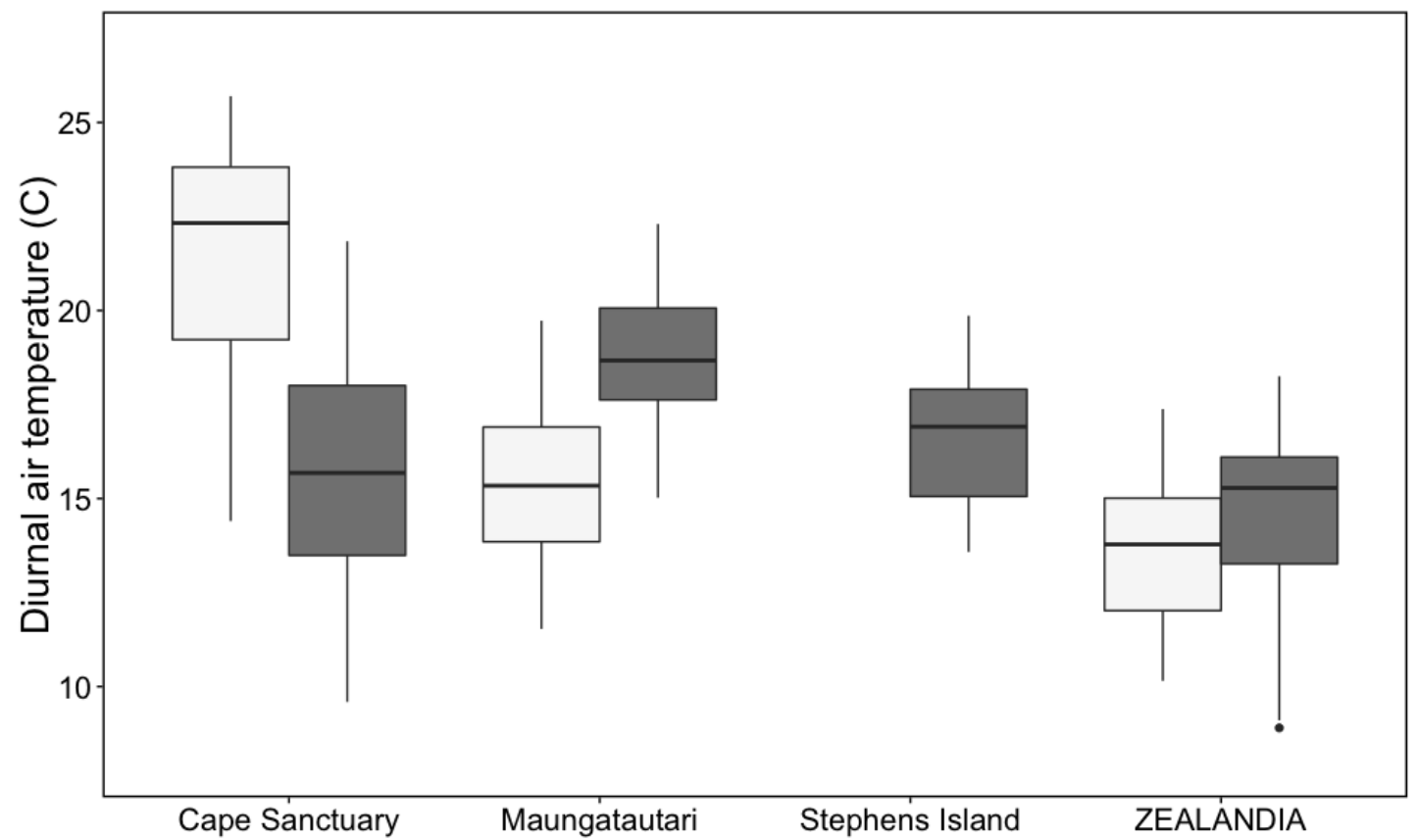

Figure 5.10 Boxplots showing range of diurnal air temperatures recorded at observational time points over 7 days per site for the spring 2014 (white) and autumn 2015 (dark grey) thermoregulatory behaviour surveys. Boxplots show the median and interquartile range and whiskers extend up to 1.5 times the interquartile range. No temperature data are shown for Stephens Island in spring 2014 as the site was not visited in that season. Black dot shows an outlier in the autumn 2015 data. Temperature data were obtained from one HOBO datalogger per site. 
Table 5.4 Results of best-fit GLMs for spring 2014 diurnal emergence data. The best-fit model for the spring 2014 Maungatautari emergence data was not a significant improvement over the null so has not been included. Model p-value indicates improvement of the model over the null model. P-value indicates significance of the individual fixed effect.

\begin{tabular}{cccccccccc}
\hline \multirow{2}{*}{ Season } & \multicolumn{1}{c}{ Site } & $\begin{array}{c}\text { Model fixed } \\
\text { effect }\end{array}$ & Model $\mathbf{X}^{2}$ & $\begin{array}{c}\text { Model } \mathbf{X}^{2} \\
\text { prob. }\end{array}$ & df & estimate & Std. error & z-value & p-value \\
\hline \multirow{2}{*}{ Spring 2014 } & Cape Sanctuary & Temperature & 10.252 & 0.001 & 1 & 0.169 & 0.055 & -3.097 & 0.002 \\
& ZEALANDIA & Temperature & 10.134 & 0.001 & 1 & 0.444 & 0.148 & 3.009 & 0.003 \\
\hline & Cape Sanctuary & Temperature & 5.746 & 0.017 & 1 & 0.158 & 0.066 & 2.412 & 0.016 \\
& Maungatautari & Temperature & 10.401 & 0.001 & 1 & 0.331 & 0.108 & 3.059 & 0.002 \\
\multirow{2}{*}{ Autumn 2015 } & Stephens Island & Temperature & 17.835 & $<0.001$ & 1 & 0.453 & 0.113 & 4.025 & $<0.001$ \\
& ZEALANDIA & Temperature & 33.057 & $<0.001$ & 1 & 0.572 & 0.120 & 4.773 & $<0.001$ \\
\hline
\end{tabular}



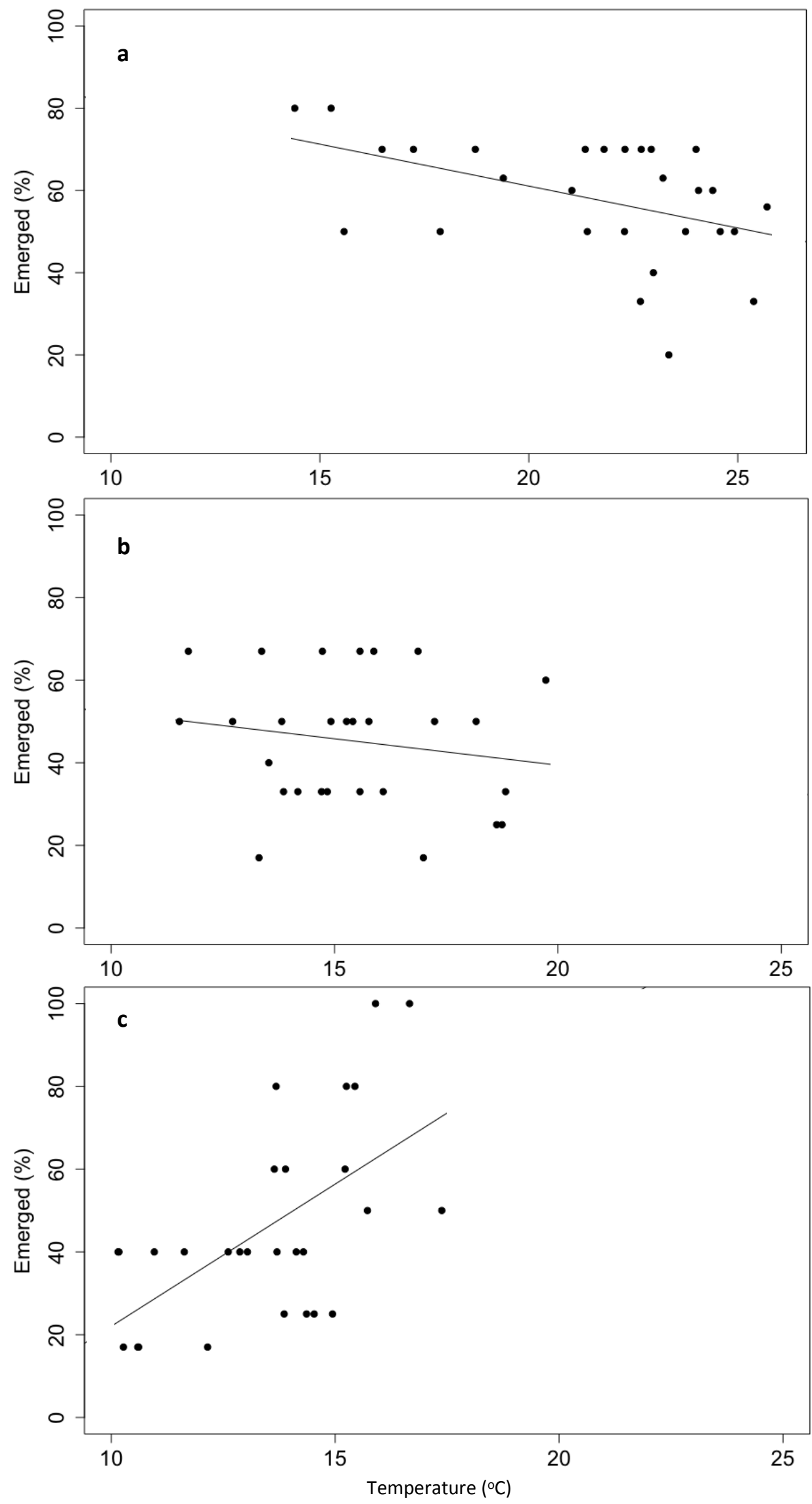

Figure 5.11 Spring 2014 diurnal emergence of tuatara by air temperature per site. a = Cape Sanctuary; $b=$ Maungatautari; $c=$ ZEALANDIA. No significant effect of air temperature on emergence was observed in the Maungatautari population. Line shows the best fit line. 

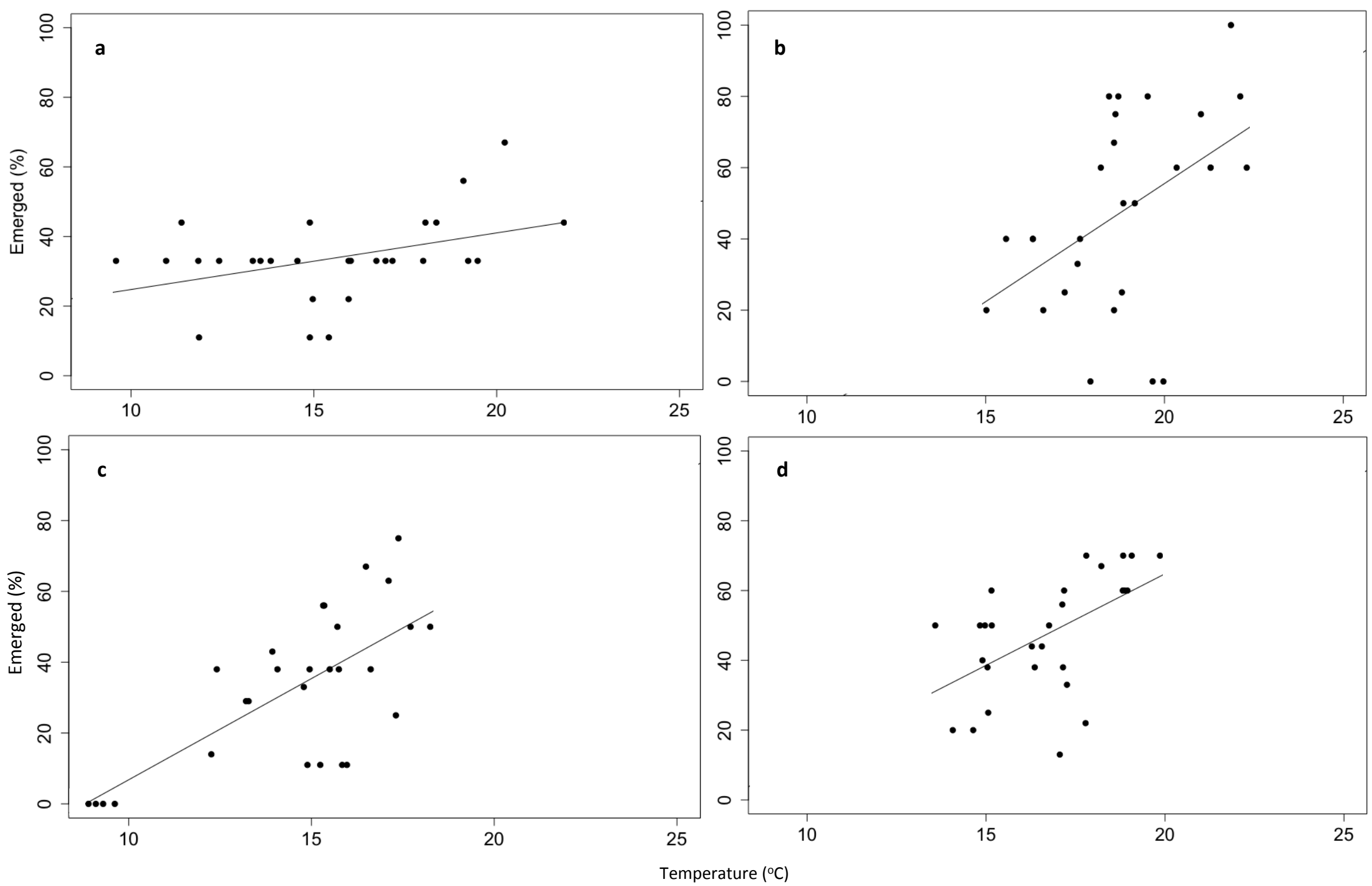

Figure 5.12 Autumn 2015 diurnal emergence of tuatara by air temperature per site. $a=$ Cape Sanctuary; $b=$ Maungatautari; $c=$ ZEALANDIA, $d=$ Stephens Island. Line shows the best fit line. 


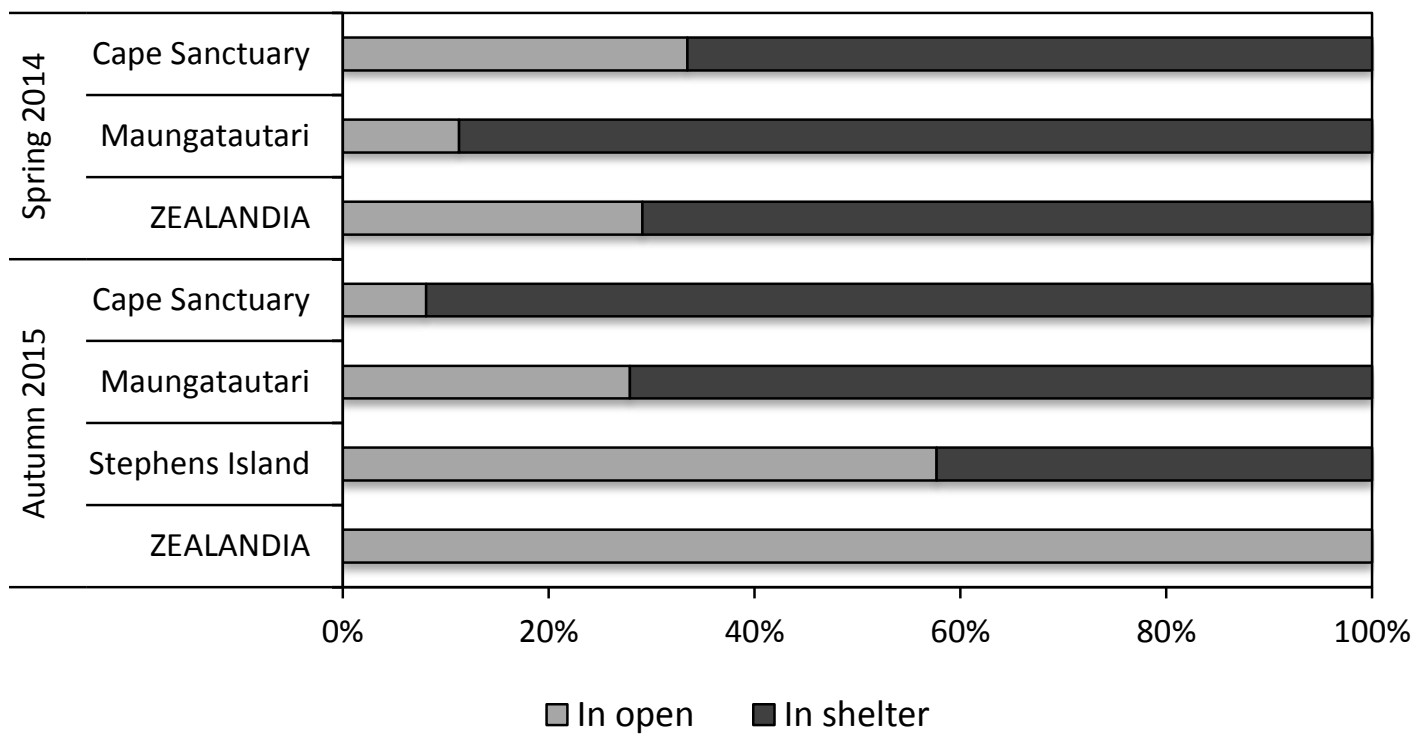

Figure 5.13 Proportion of the total diurnal out-of-burrow observations in which tuatara were recorded as positioned in the open or in shelter.

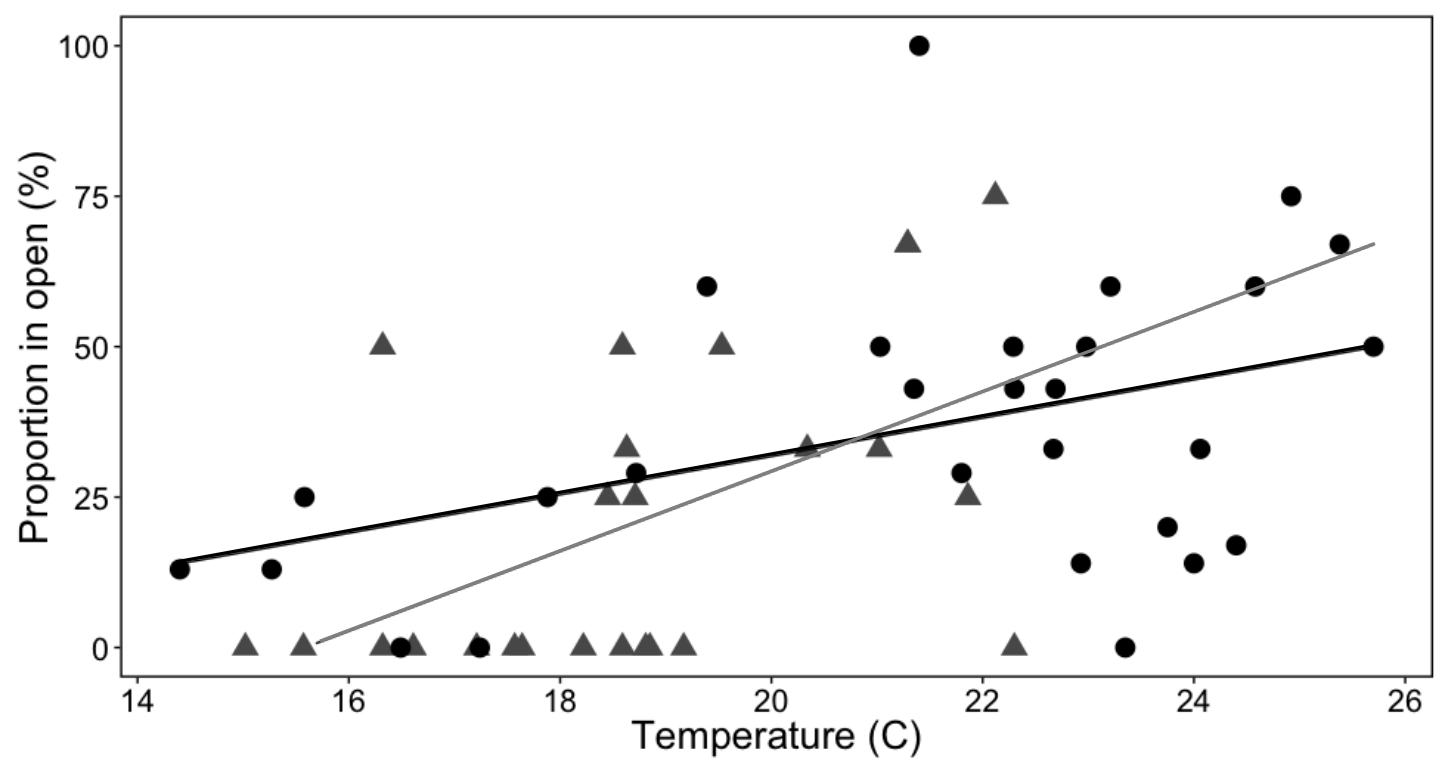

Figure 5.14 Significant relationship between air temperature and proportion of tuatara in open vs. in shelter when outside of burrows. Lines show the best fit (regression) line per population (black line = Cape Sanctuary, grey line = Maungatautari. $\bullet$ = Cape Sanctuary, $\mathbf{\Delta}$ = Maungatautari. 


\subsection{Discussion}

\subsubsection{Environmental and skin temperatures}

The thermal climate of a site can exert a significant influence on the thermoregulatory behaviour and activity of ectotherms, which in turn influences body temperature, physiological processes, and fitness-related behaviours crucial to individual survival and population viability (Angilletta 2009). I found evidence that environmental temperatures significantly influenced both nocturnal activity and diurnal thermoregulatory behaviour, with higher air temperatures found to enable increased overnight emergence and greater movement, as well as affecting daytime emergence and location in the out-of-burrow environment.

I found significant correlations between tuatara skin temperature and a broad range of air temperatures at each of the sites studied. These findings indicate that tuatara skin temperatures varied in response to air temperature and are in agreement with previous studies that found tuatara to be a more thermoconformatory than precisely thermoregulatory species (Saint Girons 1980; Saint Girons et al. 1980; Thompson \& Daugherty 1998; Walls 1983). It is unfortunate that this relationship could only be assessed in May, a comparatively cooler month (austral autumn), and it would be useful to investigate this relationship in the hotter austral summer season (December to February), when air temperatures are more likely to exceed the tuatara activity temperature range, to see if this thermoconformatory pattern is upheld.

The comparatively weaker skin-air temperature associations calculated for the Maungatautari and ZEALANDIA tuatara indicate that skin temperatures did not relate as closely to air temperatures at these sites in the period studied (May 2014). From closer inspection of the data I saw that these two sites experienced cooler air temperatures than Cape Sanctuary and Stephens Island, and it was at these lower air temperatures (below $10^{\circ} \mathrm{C}$ ) that the association between skin and air temperature was less apparent and tuatara were found to maintain warmer skin than air temperatures, with skin temperatures seen to drop no lower than $8-12^{\circ} \mathrm{C}$ and burrow temperatures dropping to minimums of $8-10^{\circ} \mathrm{C}$ at low air temperatures. Active tuatara have been recorded as having body temperatures ranging from $5^{\circ} \mathrm{C}$ up to recorded maxima of $30-34.5^{\circ} \mathrm{C}$ and Saint 
Girons (1980) suggested the minimum temperature voluntarily tolerated is around $6{ }^{\circ} \mathrm{C}$, therefore I suggest that tuatara at Maungatautari and ZEALANDIA exhibited more active thermoregulatory behaviour and likely sought shelter in comparatively warmer burrows when air temperatures dropped below $\sim 5^{\circ} \mathrm{C}$, thereby enabling the maintenance of the warmer skin temperatures recorded (Saint Girons 1980; Saint Girons et al. 1980; Barwick 1982; Thompson \& Daugherty 1998; Corkery 2012). Similarly, Cape Sanctuary tuatara exhibited warmer skin temperatures at lower air temperatures and cooler skin temperatures at higher air temperatures approaching and exceeding $20^{\circ} \mathrm{C}$, which again indicates that there are cooler and warmer air temperatures at which these tuatara became more active thermoregulators and potentially sought refuge in comparatively warmer/cooler burrows. This demonstrates that these tuatara were able to actively modify their thermoregulatory behaviour in response to the immediate climate and keep their skin temperatures elevated/cool when air temperatures were less suitable, but were largely thermoconformatory at air temperatures between approximately 10 and $15^{\circ} \mathrm{C}$. Similarly, previous work by Vermunt et al. (2014) found that captive juvenile tuatara adjusted their emergence and basking behaviour in response to experimental temperatures, emerging exclusively during the day and basking for significantly longer at low air temperatures $\left(5^{\circ} \mathrm{C}\right)$ when compared to the warmer treatments $\left(12^{\circ} \mathrm{C}\right.$ and $\left.20^{\circ} \mathrm{C}\right)$ at which activity was diurno-nocturnal.

The weaker associations observed at Maungatautari and ZEALANDIA in particular, where tuatara were shown to maintain comparatively higher skin than air temperatures, suggests that these tuatara may have been exploiting the thermal environments available to them more effectively and thermoregulating more precisely in response to the lower thermal quality of the environment, a behaviour previously reported by Besson and Cree (2010) who found that captive tuatara were more accurate thermoregulators when in a low-quality versus high-quality thermal habitat (i.e. when basking opportunities were reduced). Similarly, Jarvie et al. (2015) found that juvenile tuatara translocated to or head started at Orokonui Ecosanctuary, which has a much cooler climate, were able to achieve body temperatures comparable to those seen in Stephens Island tuatara in summer. Further evidence for this also comes from the lower skin temperatures observed in the Stephens Island tuatara in the same period. While Stephens Island did not experience the lowest temperatures of the four sites studied in May 2014, the female tuatara monitored 
at this site displayed the lowest ranges of minimum and mean skin temperatures, suggesting that females in this population were not thermoregulating as precisely as females in the colder Maungatautari or ZEALANDIA populations. The thermoregulation cost-benefit model proposed by Huey and Slatkin (1976) suggests that ectotherms will stop thermoregulating when the costs outweigh the benefits. This suggests that the benefits to the Maungatautari and ZEALANDIA tuatara of retreating to warmer burrows at low air temperatures outweighed any costs associated with the animal's consequent inactivity, whereas for the Stephens Island tuatara the benefits of remaining active in the outside environment were greater than those associated with retreating to a warmer burrow, resulting in the lower skin temperatures seen.

As thermoregulation is a time-consuming behaviour (i.e. time is spent shuttling between warmer and cooler microclimates) this suggests that tuatara at sites that experience a higher frequency of low air temperatures throughout the year will spend more time thermoregulating, potentially increasing their vulnerability to predation (via increased visibility when basking) and leaving less time available for fitness-related behaviours like foraging, territory defense and mating (due to potentially increased time spent in burrows at low air temperatures), which could place them at a disadvantage in comparison to populations that experience warmer year-round climates (DeWitt 1967; Pianka \& Pianka 1970; Huey \& Slatkin 1976; Huey 1982; Besson \& Cree 2010). However, in the absence of data on how much more or less costly thermoregulation is at the different sites (in terms of time and energy lost) and how this might translate to overall fitness, I cannot quantify to what extent tuatara at warmer sites like Cape Sanctuary might benefit from increased behavioural opportunities when compared to a cooler site like ZEALANDIA (Huey 1974). However, I do know that Cape Sanctuary tuatara were often observed in shelter when outside of their burrows, perhaps offsetting costs of emergence at warmer temperatures or in comparatively exposed habitat (i.e. with less closed canopy cover), which would impose a constraint on time available for activities like foraging.

Our decision to calculate degree days using $10^{\circ} \mathrm{C}$ as the baseline temperature was based on observations of diurnal emergence made during this study, as well as similar observations made in a study by Mello et al. (2013). Although tuatara have been observed above ground at lower nocturnal air temperatures I wanted to select a reasonably conservative emergence temperature that would apply to both diurnal and nocturnal 
emergence. The higher numbers of $>10^{\circ} \mathrm{C}$ and $>19^{\circ} \mathrm{C}$ degree days consistently recorded at the translocation sites Cape Sanctuary, Young Nicks Head and Whangaokeno, demonstrate that these sites experienced warmer climates year-round than the source site (Stephens Island), control site (ZEALANDIA) and Maungatautari. In comparison, although Maungatautari is located at a lower latitude than the founder and control sites, which would usually be associated with a consequently warmer climate, its higher elevation and inland location has resulted in a more continental climate that is comparable to Stephens Island and ZEALANDIA for much of the year, but with higher numbers of $>10^{\circ} \mathrm{C}$ and $>19^{\circ} \mathrm{C}$ degree days throughout summer (December - February) and into autumn.

The warmer year-round climates at the translocation sites Cape Sanctuary, Young Nicks Head and Whangaokeno will likely benefit tuatara populations through increased emergence opportunities throughout the year and further into the winter season (i.e. May onwards), reducing the temperature-dependent suppression of activity associated with winter temperatures at cooler sites, though observations on tuatara activity leading into and during winter are required to confirm this. Increased incidence of $>10^{\circ} \mathrm{C}$ and $>19^{\circ} \mathrm{C}$ days at these higher thermal quality sites and consequently increased emergence opportunities could also facilitate enhanced hunting/foraging behaviour (which occurs between $8^{\circ} \mathrm{C}$ and $20^{\circ} \mathrm{C}$ ), enhanced digestion throughout winter (which occurs at temperatures of $12^{\circ} \mathrm{C}$ and above) and reduce time spent actively thermoregulating, allowing more time and energy for fitness-related behaviours like reproduction, territory defence and predator avoidance (Huey 1974, 1982; Huey \& Slatkin 1976; Saint Girons 1980; Seebacher 1999; Besson \& Cree 2010, 2011). It should also be noted here that, while the preferred body temperature range of adult females is unknown and in this study was based on recorded preferences of adult males and unsexed juveniles, it is feasible that the reproductive condition of females might influence their thermal preferences towards higher temperatures when going through oogenesis (i.e. producing eggs; Cree 2014), in which case warmer climates could potentially benefit reproductive process in females. Equally, the warmer year-round climates and increased digestive and foraging opportunities could also lead to enhanced temperature-dependent growth rates in these translocated populations (Autumn \& De Nardo 1995; Avery et al. 1982; Christian \& Tracy 1981; Corkery et al. 2014). However, these warmer sites, Cape Sanctuary in particular, 
also experienced higher numbers of $>25^{\circ} \mathrm{C}$ days, at which emergence from burrows may be constrained by the higher air temperatures, thereby reducing opportunities for activity and fitness-associated behaviours described previously and limiting the potential benefits associated with translocation to a warmer site. In the context of the thermal climates available to tuatara it is also important to note that, at both cooler and warmer sites, burrows consistently provided cool refuges from high temperatures and warm refuges from cool temperatures, enabling tuatara to avoid hazardous minimum $\left(0.7^{\circ} \mathrm{C}\right)$, approximate voluntary maximum $\left(\sim 26-30^{\circ} \mathrm{C}\right)$, and upper lethal $\left(34.5^{\circ} \mathrm{C}\right)$ temperatures throughout the year (Wilson \& Lee 1970; Barwick 1982; Blanchard 2002; Besson \& Cree 2011; Cree 2014).

In contrast, the cooler sites Maungatautari, Stephens Island and ZEALANDIA experienced substantially fewer degree days favourable to burrow emergence or the attainment of preferred body temperatures, suggesting that these populations may be at a comparative disadvantage regarding emergence, digestion and opportunities for other fitness-related behaviours throughout the year. Corkery (2012) also found that the thermal environment on Stephens Island between 2008 and 2011 rarely offered tuatara the opportunity to achieve body temperatures within their preferred range, indicating that this primarily thermoconformatory population is able to survive under suboptimal temperatures. The reduced emergence opportunities over winter also suggests that the tuatara translocated to Maungatautari will undergo similar periods of suppressed activity seen in the Stephens Island and ZEALANDIA populations (Walls 1983; Seebacher 2005). However, given the higher year-round mean relative humidities for the regions these sites occupy and the lower/lack of $>25^{\circ} \mathrm{C}$ degree days these populations might benefit from an absence of potentially restrictive high temperatures, which could enable greater activity throughout summer in comparison to a site like Cape Sanctuary, which experiences lower relative humidity and rainfall over summer and higher numbers of $>25^{\circ} \mathrm{C}$ days.

\subsubsection{Nocturnal emergence and activity}

Tuatara emergence was recorded on nights under all mean overnight temperatures recorded in this study, from as low as $7.7^{\circ} \mathrm{C}$ up to the maximum recorded mean temperature of $19.2^{\circ} \mathrm{C}$. Other studies also found tuatara to be nocturnally active at 
similarly low temperatures, with Walls (1983) reporting above-ground activity at around $7-8^{\circ} \mathrm{C}$ and Saint Girons (1980) reporting a minimum voluntary temperature of $\sim 6^{\circ} \mathrm{C}$. Nocturnal emergence, net distance moved overnight and displacement from the burrow were positively related to mean overnight temperatures in spring 2014, with greater proportions of the monitored tuatara found to have emerged from burrows and moved more and further in the environment at warmer overnight temperatures. Emergence and net distance moved in the environment were also positively influenced by mean overnight temperature in autumn 2015, whereas displacement was not. I found few other studies on the nocturnal activity of tuatara in the literature, potentially due to the difficulties associated with inconspicuous overnight monitoring, however our findings are in agreement with previous work by Walls (1983) who also found nocturnal emergence and above-ground movement of tuatara and other New Zealand reptiles to be temperaturedependent, with overall greater activity and greater numbers of tuatara, skinks and geckos observed in warmer months. However, it is important to note that in looking at emergence/activity in relation to mean overnight temperatures and not temperatures at the time of emergence/activity, we may have missed more detailed behaviours (i.e. on cooler nights emergence may have occurred earlier, while air temperatures were warmer). Specifics around air temperature and the time of emergence could be studied through the use of PIT tag scanners placed at burrow entrances, though the large number of burrows utilised by the monitored females made this method impractical and the spooling method used was considered to be the most appropriate alternative. Despite the positive relationship between temperature and nocturnal activity and the significantly warmer nocturnal temperatures experienced at Cape Sanctuary in spring 2014, this did not translate to significantly greater emergence or movement of tuatara at this site, which could be attributed to relatively high levels of emergence across the sites surveyed and substantial within-site variability in distances moved by individuals. Alternatively, a limiting factor such as humidity or barriers to movement (e.g. other tuatara) could have constrained the movement of Cape Sanctuary tuatara in the environment, leading to comparatively lower distances moved than would have been possible in the absence of this restraint.

Conversely, significant between-site differences in net distances moved were observed in autumn 2015, with tuatara at the warmest sites (i.e. Maungatautari and 
Stephens Island) found to have moved significantly greater distances than those at the coolest site (i.e. Cape Sanctuary). Given that warmer body temperatures enhance physiological process that influence locomotor function in reptiles, the greater distances moved at Maungatautari and Stephens Island are likely due to the warm air temperatures enabling females to move further overnight (Bennett 1980; Christian \& Tracy 1981; Huey 1982). Similarly, the comparatively cooler temperatures experienced at Cape Sanctuary and ZEALANDIA likely resulted in the shorter distances moved. It is also important to note that despite the higher range of overnight temperatures recorded at Stephens Island, the net distances moved at this site were significantly lower than those seen at Maungatautari and comparable to those seen at the cooler ZEALANDIA site. As with the spring 2014 Cape Sanctuary results, this suggests the presence of an alternative limiting factor restricting movement on Stephens Island (e.g. high population density in forest habitat; Moore et al. 2007).

Although Walls (1983) found air temperature to be the most important variable influencing nocturnal activity, it was also noted that relative humidity extremes were influential, with numbers of emerged tuatara greatly reduced on very dry nights and tuatara most numerous and active on warm nights when it rained. This suggests that temperature is the most important factor influencing tuatara activity, with the caveat that humidity is sufficiently high. In support of this, a laboratory-based study by Corkery et al. (2014) also found that the number of juvenile tuatara outside of burrows was greater under humid (55.7-70.8\% RH) versus dry (35.1-51.7\% RH) conditions. While RH may have been a limiting factor influencing nocturnal emergence and movement, I was unfortunately unable to assess $\mathrm{RH}$ levels at the sites I studied due to irregular $\mathrm{RH}$ readings obtained by the dataloggers used, rendering all $\mathrm{RH}$ readings unreliable. However, mean monthly $\mathrm{RH}$ data obtained from nearby weather stations gave us an indication of the RH levels experienced at study sites throughout the year and demonstrated Cape Sanctuary to be a comparatively drier site year-round (Chapter Two, Figure 2.2). The lowest mean RH recorded for Cape Sanctuary in 2014 was 62.3\%, which falls within the "humid" condition range described by Corkery et al. (2014), suggesting that while very low RH can constrain tuatara behaviour, humidities are not so low at the comparatively drier sites that behaviour should be severely restricted. That said, given that Walls (1983) recorded dry nights and substantially reduced activity on Stephens Island (a comparatively humid 
site) it is likely that the drier translocation sites will experience greater incidences of suboptimal humidities and consequently reduced tuatara activity during these dry periods.

Equally, while population density was not found to have a significant influence on net distance or displacement in any of the populations studied, it is feasible that, given the territorial nature of tuatara and the fact that home ranges have been found to overlap with neighbours' territories, the distances moved away from burrows and around in the environment might be limited by proximity of neighbouring tuatara and the avoidance of potential conflict, which may not be regulated by population density alone, but also the availability of preferred habitat and resources (i.e. food and refuge) as seen in differing densities between forest ( 2,700 per hectare) and pasture ( 200 per hectare) habitat on Stephens Island (Stamps 1994; Gillingham et al. 1995; Moore et al. 2009a, 2009b). Also, as tuatara forage primarily at night it should be considered that individual emergence and distances moved might be motivated in part by an animal's hunger state, though I was not able to find any studies in the literature on hunger state influencing activity in reptiles (Besson \& Cree 2010). Given that both Cape Sanctuary and Stephens Island experienced reasonable monthly mean $\mathrm{RH}$ levels (Cape Sanctuary: 62\%, Stephens Island: $87 \%$ (http://www.cliflo.niwa.co.nz)) around the time behaviour was observed and significantly warmer climates than sites with comparable levels of activity, I suggest that the lowerthan-expected activity seen at Cape Sanctuary in spring 2014 and Stephens Island in autumn 2015 could have been restricted by the proximity of other tuatara, which imposed an upper limit on the distances tuatara were able to roam at air temperatures that were conducive to greater movement. I calculated the population density on Stephens Island to range between 200 and 333 individuals per hectare (based on site size and population estimates), however previous work has found population density in areas of remnant forest (e.g. Keepers Bush, an area in which we observed females) to be closer to 2,700 per hectare, over five times greater than the density seen in pasture habitat ( 200 per hectare) on the island and suggestive of very small home ranges within this area, which could impose a limit on the distance and displacement of tuatara in this habitat (Carmichael et al. 1989; Moore et al. 2009a, 2009b). Data from previous studies showing small territory size and extensive spatial overlap with neighbouring tuatara in the Stephens Island population, especially of the subordinate females, also suggests that this 
is a plausible explanation; however, in the absence of site-specific data on home range size and connectivity of the Cape Sanctuary individuals we are unable to confirm this (Moore et al. 2009a; Godfrey et al. 2010b).

\subsubsection{Diurnal emergence and behaviour}

In agreement with previous work on the diurnal thermoregulatory behaviour of tuatara we found that variable but often large proportions ( $20-100 \%)$ of the tuatara observed were seen above ground during daylight hours (Walls 1983). Tuatara are considered partially diurnal and emerge from burrows during the day to take advantage of extra opportunities to engage in basking, feeding, mating and defensive behaviours and are more likely to obtain body temperatures around their preferred range when exposed to warmer daytime climates (Besson \& Cree 2010; Gillingham et al. 1995; Saint Girons et al. 1980; Walls 1981). However, as higher air temperatures can lead to evaporative water loss, which can lower body temperature, many animals are thought to minimise the risk of water loss by remaining in burrows for much of the day and, correspondingly, we observed that the proportions of emerged tuatara were generally greater at night (Bogert 1949; Walls 1983).

As with nocturnal activity, we found that diurnal emergence was positively associated with air temperature, a pattern also observed in juvenile tuatara (Mello et al. 2013). Owing to the range of diurnal temperatures recorded at Cape Sanctuary in spring 2014 and ZEALANDIA in autumn 2015 we were able to discern possible upper and lower limits to diurnal emergence. The only two sites to experience air temperatures below $10^{\circ} \mathrm{C}$ during behavioural observations were ZEALANDIA and Cape Sanctuary, and while 33\% of the Cape Sanctuary tuatara were above-ground on one occasion at $9.59^{\circ} \mathrm{C}$ none of the ZEALANDIA tuatara were seen outside of burrows below $10^{\circ} \mathrm{C}$. The weaker association between emergence and air temperature observed at Cape Sanctuary in this season (autumn 2015) also suggests that an alternative factor such as humidity may have influenced diurnal emergence at this site (though in the absence of humidity data we cannot be certain), therefore we took $10^{\circ} \mathrm{C}$ to be the approximate lower limit above which tuatara emerged from burrows during the day (Walls 1983). A similar lower limit for diurnal emergence was established by Mello et al. (2013), who found that emergence 
from burrows was not observed at temperatures of $10.5^{\circ} \mathrm{C}$ or below. The highest diurnal temperatures documented during behavioural monitoring were recorded at Cape Sanctuary in spring 2014 , where temperatures ranged from $14-26^{\circ} \mathrm{C}$. This was the only instance in which diurnal emergence showed a negative association with temperature, with emergence found to be relatively stable between $14^{\circ} \mathrm{C}$ and $23^{\circ} \mathrm{C}$, but declined as temperatures approached $25^{\circ} \mathrm{C}$. While we did not record an upper limit at which zero emergence was observed, this suggests that diurnal emergence is likely low at air temperatures above $26^{\circ} \mathrm{C}$, which is in agreement with previous work that has shown basking tuatara to reach body temperatures of $25-26^{\circ} \mathrm{C}$ and up to a maximum of $30^{\circ} \mathrm{C}$ (Barwick 1982; Saint Girons et al. 1980). However, in the absence of a broader range of recorded temperatures per study site we are unable to say if these potential emergence minima and maxima might be site specific or applicable to tuatara populations in general and how it might interact with humidity.

We rarely found tuatara observed outside of burrows to be basking in full or partial sun, which contrasts with previous work that witnessed tuatara basking in or tracking patches of sunlight throughout the day (Corkery et al. 2014; Mello et al. 2013; Walls 1983). Instead, in the majority of observations at Cape Sanctuary and Maungatautari I found tuatara in shelters, largely obscured from the view of the observer and, while many ZEALANDIA tuatara were in shelter when observed in spring 2014, in autumn 2015 the majority of Stephens Island and ZEALANDIA tuatara were observed in the open (i.e. outside of burrows and visible in the environment), but under the shade of the forest canopy. Although air temperature was only found to influence out-of-burrow behaviour at the warmest sites studied in each survey season (i.e. Cape Sanctuary in spring 2014 and Maungatautari in autumn 2015), we suggest that the behaviour observed at other sites could be attributed in part to climate but also to habitat variation among sites, with greater proportions of "in the open" observations made at sites that were primarily closed canopy forest. Previous studies have found similar results, with tuatara observed basking in the open when under the cover of forest canopy while animals observed in pasture opted to bask in protected retreats (aka shelters; Werner \& Whitaker 1978; Gans 1983). However, a large male tuatara has been observed basking in the open in pasture on Stephens Island, though in close proximity to its burrow (Cree 2014). Accordingly, mature forest covers large areas of both Stephens Island and ZEALANDIA, whilst Cape Sanctuary 
is primarily regenerating bush (e.g. Coprosma sp., Phormium tenax) and grassland, with little closed forest canopy (though dense flax does provide some low canopy cover at this site), and the Maungatautari Tautari Wetland is partially closed regenerating canopy, with open areas of bare soil as well as regenerating vegetation (e.g. P. tenax, ferns and Coprosma sp.).

In support of the potential role of air temperature, when looking at above-ground behaviour within sites we noted a greater proportion of observations showed tuatara in the open in the warmer of the two surveys (e.g. during the cooler spring 2014 survey at ZEALANDIA $29 \%$ of observations showed tuatara in the open, compared to $100 \%$ in the warmer autumn 2015 survey season). The same trend can be seen in the other sites studied though a significant effect of temperature was only found at sites that experienced diurnal air temperatures above $\sim 19^{\circ} \mathrm{C}$, with greater proportions of tuatara observed in the open at higher temperatures. This result was contrary to the pattern of habitat selection that we expected as we thought tuatara at Cape Sanctuary and Maungatautari might consistently select shelter over open ground to mitigate predation risk while out of the burrow and minimise water loss at higher temperatures. However, it is possible that the benefits associated with attainment of body temperatures within and above the tuatara's preferred range were outweighed by any risks associated with leaving shelter to bask in the open. However, as tuatara observed in the open were rarely far from a burrow it appears that behaviour when in the open still acted to minimise predation risk. This indicates that tuatara likely adjust their diurnal thermoregulatory behaviour in response to both the climate and habitat structure of their environment to take advantage of thermoregulatory opportunities whilst minimising predation risk.

However, tuatara also make cost-benefit trade-offs between safety from predation and the opportunity to obtain skin temperatures within their preferred body temperature range, modifying their behaviour when air temperatures climb above $19^{\circ} \mathrm{C}$. Varying strength of correlations between skin and air temperature and comparisons of emergence at warmer and cooler sites during the 1100 observations also provide limited evidence that tuatara may have thermoregulated less precisely when sites were warmer/of higher thermal quality (Besson \& Cree 2010). Females observed at Cape Sanctuary in spring 2014 did not show high levels of emergence at 1100 when air temperatures were within or above their preferred temperature range, which was not 
explained by burrow temperatures enabling attainment of preferred body temperatures. This led to the conclusion that emergence of some females may have been restricted or that females thermoregulated less precisely because of the environment's high thermal quality. Observations of zero emergence at Maungatautari (the warmest autumn 2015 site) when air temperature was within the preferred range lends some small support to this hypothesis; however, it would be necessary to obtain a greater number of thermoregulatory observations over a broader range of temperatures at high and low thermal quality sites to properly assess this.

\subsubsection{Conclusions and conservation implications}

The consistently warmer temperatures of several translocation sites, the close association between air and skin temperatures and the generally positive impact of air temperature on emergence and activity suggests that tuatara translocated to warmer regions may benefit from more frequent attainment of preferred body temperatures, more time at preferred body temperatures, and greater emergence opportunities throughout the year. Other benefits may include a reduction in the time and energy spent on thermoregulation and a consequent increase in the time and energy that can be spent on other fitnessrelated activities, which could benefit individual survival and reproductive success and ultimately improve population viability. As burrows were consistently shown to provide refuge from both high and low temperature extremes and tuatara were found to show decreased emergence at low and high temperatures (instead remaining in burrows) we have no reason to believe that tuatara translocated to warmer sites like Whangaokeno, Young Nicks Head and Cape Sanctuary will be at risk of attaining body temperatures close to their critical thermal limits under the current climate, though the comparatively drier

climates of Cape Sanctuary and Young Nicks Head (and potentially Whangaokeno) could be problematic in summer months and restrict emergence and fitness-related behaviours.

I was unable to assess environmental or burrow humidity at sites in this study due to unreliable equipment. However, Corkery (2012) found that the relative humidities of burrows on Stephens Island ranged between $64 \%$ and $100 \%$, with significantly higher humidities recorded in burrows occupied by fairy prions (Pachyptila turtur), which have been seen to share burrows with tuatara on Stephens Island. This suggests that burrows 
likely provide refuges from unsuitable temperature and humidity and could have positive implications for tuatara at sites like Whangaokeno, which is home to large colonies of burrowing seabirds. However site-specific $\mathrm{RH}$ data are needed to ascertain if low RH is a realistic concern and further work to assess the relationship between environmental humidity, burrow humidity and behaviour is required to determine the extent to which humidity might limit emergence and activity of wild tuatara at drier sites and the extent to which burrows may provide refuges from dry environments.

Further work is also required to assess the upper and lower thermal limits to emergence and activity at each site as a sufficient range of air temperatures was not obtained per site in the course of this study, which a longer term study assessing behaviour over multiple seasons would likely achieve. This would help establish whether the thermal limitations we observed were responses to site-specific climates or broadly reflective of tuatara physiology and therefore generalizable to all tuatara populations. More frequent or constant observations of tuatara behaviour would also be of use to determine finer scale details of factors influencing emergence and movement in the environment. For instance Mello et al. (2013) used wide-angle cameras to continuously record diurnal tuatara behaviour over 2.5 to three hour periods, obtaining detailed observations on emergence, burrow use, and movement in that time. However the juvenile tuatara observed using this method were housed in $2 \times 1 \times 0.8 \mathrm{~m}$ pens, so cameras may not be a practical method for monitoring wild tuatara in larger, more complex environments. I have also had limited success monitoring tuatara using motion/heattriggered trail cameras, possibly due to the generally low body temperatures and slow movement of tuatara. Alternatively, Corkery (2012) used PIT (Passive Integrated Transponder) tag scanners (which read identification tags implanted in tuatara - see Chapter 2) placed at burrow entrances to monitor movement of tuatara in and out of burrows, which enabled precise monitoring of burrow use in the absence of an observer, but would not provide information on activity outside of the burrow.

Regarding the potential impacts of anthropogenic climate change on tuatara populations, the results of this study suggest that tuatara may initially benefit from warming climates in the same way that those moved to warmer translocation sites will likely benefit. The IPCC (Intergovernmental Panel on Climate Change) predictions for New Zealand project air temperature increases for the North Island to be in the range of $0.2-$ 
$1.4^{\circ} \mathrm{C}$ by the $2040 \mathrm{~s}$, which are unlikely to pose a direct threat to adult tuatara survival. However, such temperature changes could affect embryonic development and bias hatchling sex ratios towards males, which could impact population demography and threaten long-term population viability (Nelson et al. 2002a, 2004b; Huey \& Janzen 2008).

Equally, projected temperature increases by the 2090s are in the range of $0.7-$ $5.1^{\circ} \mathrm{C}$ (Reisinger et al. 2014). Coupled with reduced precipitation, increased soil evaporation and increased drought predicted for Eastern New Zealand, which could also impact habitat structure at sites in the early stages of revegetation, this suggests that the populations translocated to East Coast sites like Cape Sanctuary, Young Nicks Head and Whangaokeno could eventually be threatened by adverse climatic conditions and associated risks (e.g. drought, increased fire risk, heat stress; Hennessy et al. 2007; Reisinger et al. 2014). Given the decline in emergence observed at air temperatures approaching $26^{\circ} \mathrm{C}$ and potential upper threshold temperatures of around $26-30^{\circ} \mathrm{C}$, as temperatures continue to rise then tuatara already occupying warmer climates may be particularly vulnerable and translocations to sites with cooler climates might offer a better chance of population persistence.

Several studies have demonstrated that, despite restricted thermoregulatory opportunities, tuatara translocated to Orokonui Ecosanctuary (a cooler mainland translocation site located in New Zealand's South Island) have been able to survive, grow and should be able to provide incubation environments that allow the production of both male and female offspring, especially as the climate warms (Besson \& Cree 2010; Besson et al. 2012; Mello et al. 2013; Jarvie et al. 2014, 2015). Likewise, this suggests that tuatara populations at the cooler sites Maungatautari, ZEALANDIA and Stephens Island may benefit from rising air temperatures as climate change progresses. That being said, behavioural adjustments like selection of basking habitat, a decrease in diurnal emergence or changes in patterns of seasonal activity (i.e. reduced activity over summer rather than winter) might offer a means by which tuatara at warmer sites can mitigate risks associated with the occupation of warmer climates. Studies on thermoregulatory behaviour over warmer summer months at site like Whangaokeno, Young Nicks Head and Cape Sanctuary could offer insight into mechanisms by which tuatara might adjust to the hotter, drier climates predicted for regions like New Zealand's East Coast and how this 
lineage may have survived through past climate change events (Hennessy et al. 2007; Reisinger et al. 2014). 


\section{Chapter Six}

\section{Will a warming climate produce "smarter" reptiles? Ambient temperature and learning performance in the tuatara (Sphenodon punctatus)}

\subsection{Introduction}

Learning is a relatively permanent change in a behaviour as a direct result of experience (Shettleworth 2010). Spatial learning in particular is a crucial cognitive ability for many reptile species, and the majority of cognitive research in these species has focused on spatial learning abilities (Burghardt 1977; Wilkinson \& Huber 2012; Carazo et al. 2014). The capacity to quickly and flexibly navigate to nest sites, territorial boundaries, refuges, potential prey, and other resources means that spatial learning underpins behaviours such as predator avoidance, foraging, reproduction, and territoriality, which in turn influence individual fitness and survival (Cooper \& Wilson 2007; Noble et al. 2012; Burghardt 2013; Carazo et al. 2014).

While the majority of animal learning studies focus on mammals and birds, reptiles have been shown to perform successfully across a range of learning paradigms, despite being previously thought to be behaviourally deficient and capable of minimal learning (Burghardt 1977, 2013; Macphail 1982; Schall 2000; Paradis \& Cabanac 2004; Woo et al. 2009; Wilkinson et al. 2010; Rodríguez-Prieto et al. 2010; Davis \& Burghardt 2011; Gaalema 2011; Wilkinson \& Huber 2012; Clark et al. 2013; Noble et al. 2014; Kis et al. 2015). There is now a body of evidence suggesting that reptiles are capable of complex behaviours and learning strategies comparable to those seen in mammalian and avian species (Kirkish et al. 1979; López et al. 2001; Manrod et al. 2008; LaDage et al. 2012; Wilkinson \& Huber 2012; Leal \& Powell 2012; Burghardt 2013).

Reptiles and other ectothermic taxa are dependent upon suitable environmental temperatures to effectively regulate body temperature and maintain normal physiological function (Deutsch et al. 2008; Hare \& Cree 2010). Several recent studies have demonstrated that egg incubation temperature can influence learning ability in some ectotherms, with "hot" incubated three-lined skinks (Bassiana duperreyi) found to be better learners than their "cold" incubated conspecifics (Amiel \& Shine 2012; Clark et 
al. 2013; Amiel et al. 2014). Similarly, warmer-incubated honey bees (Apis mellifera) displayed better short-term learning and memory than those reared at cooler temperatures (Jones et al. 2005). Amiel and Shine (2012) suggest that incubation temperature may have affected learning ability in their skinks through the modification of hormone levels or influence on endocrine pathways that affect brain development in regions like the medial and dorsal cortices (homologues of the avian and mammalian hippocampus), which control behaviours like spatial learning and memory (Ahmad \& Zemenhof 1978; Day et al. 2001; Rissman et al. 2002; López et al. 2003; Valenzuela \& Lance 2004; Amiel \& Shine 2012). The exact mechanisms through which the thermal environment during embryogenesis affects neurogenesis and learning ability in reptiles are unknown, but in humans and other animals, including reptiles, there is evidence of temperature having direct action on neural cell development, and affecting cognition through epigenetic modifiers (Penafiel et al. 2001; Radmilovich et al. 2003; Wang et al. 2012; Lin et al. 2014; Torson et al. 2015; Maynard et al. 2016). Accordingly, recent work has shown that incubation temperature can affect forebrain development in B. duperreyi, which could explain the differences in learning ability observed (Amiel et al. 2016). The authors postulate that the enhanced learning ability of warmer-incubated hatchlings may facilitate a more successful response to novel environmental change through improved behavioural flexibility (Amiel \& Shine 2012).

The relationship between cognitive ability and ambient test temperature has been investigated, but the majority of research has focused on mammalian, specifically human, cognition and the association between cognitive ability and heat stress (Blockley \& Lyman 1950; Chiles 1958; Mackworth 1961; Wing 1965; Grether 1973; Ramsey et al. 1975; Allen \& Fischer 1978; Hancock 1981, 1982; Ramsey \& Kwon 1992; Hancock \& Vasmatzidis 2003). Despite this bias, there have been several studies using ectothermic taxa that have found an effect of ambient test temperature on learning ability. Goldfish (Carassius auratus) and carp (Cyprinus carpio) were found to demonstrate temperature-dependent learning and retention on maze, swimming, and avoidance tasks (French 1942; Andry et al. 1971; Zerbolio 1973; Borsook et al. 1978). Similarly, an experiment by Krekorian et al. (1968) on desert iguanas (Dipsosaurus dorsalis) found that animals trained at the lowest temperature $\left(22^{\circ} \mathrm{C}\right)$ were unable to solve a maze task whilst those trained at the highest temperature $\left(32^{\circ} \mathrm{C}\right)$ were the most successful learners. Body temperature has also been 
found to influence neural activity in garter snakes (Thamnopsis radix and T. sirtalis), which the authors speculate might be important to learning ability (Andry et al. 1971). Within the Reptilia, the majority of cognitive studies have focused on the Chelonia, though studies on squamates and crocodilians have shown evidence of reptilian capability in most traditional learning paradigms (Burghardt 2013). However I am aware of only two studies in the literature that have investigated learning in the Order Rhynchocephalia (Northcutt \& Heath 1973; Woo et al. 2009).

Tuatara (Sphenodon punctatus) are New Zealand endemic reptiles and the sole extant representatives of the Rhynchocephalia, a lineage that arose 250 million years ago (Gaze 2001; Cree 2014). The tuatara is a medium-sized (300-1000g for adults and up to $311 \mathrm{~mm}$ snout-vent length (SVL)), nocturnal reptile with a long lifespan ( $\sim 00$ years), a cold-adapted active temperature range of $5-30^{\circ} \mathrm{C}$, and a panting threshold of $31^{\circ} \mathrm{C}$ (Bogert 1953; Wilson \& Lee 1970; Barwick 1982; Dawbin 1982; Heatwole 1982; Castanet et al. 1988; Cree \& Butler 1993; Cree 1994, 2014; Thompson \& Daugherty 1998; Gaze 2001; Miller et al. 2012). Tuatara also have a unique form of temperature dependent sex determination, type IB, in which males are produced at warmer and females produced at cooler incubation temperatures (Nelson et al. 2004a; Mitchell et al. 2006). Holocene subfossil records indicate that tuatara were once widespread throughout New Zealand, but the arrival of human settlers brought habitat change and introduced mammalian predators, which saw tuatara extirpated from the mainland and restricted to predatorfree offshore islands (Cree \& Butler 1993; Anderson 1996; Holdaway \& Worthy 1997). Conservation translocations have since served to extend the range of tuatara, but the species is still considered to be conservation dependent, range restricted, and relict (Miller et al. 2012; Hitchmough et al. 2016; Cree 2014). Only two studies have so far been published on the subject of tuatara learning ability. One found that two adult tuatara were able to learn the route to a reward in a T-maze spatial task (Northcutt \& Heath 1973) while the other trained juvenile tuatara on an operant learning task in order to examine visual sensitivity (Woo et al. 2009). Neither assessed the effect of temperature on learning. The restricted range, cold adapted lifestyle, and temperature-dependent sex determination of tuatara places them at risk from rising air temperatures (Nelson et al. 2002b; Mitchell et al. 2008). As the last living representative of a reptilian order, the tuatara is of great conservation importance and evolutionary significance (Nelson et al. 2006). 
Understanding their responses to warmer climates is necessary for direct conservation management efforts and to assure the future viability of the species.

The ability to respond quickly and effectively to novel change is of particular relevance in the face of anthropogenic climate change, which could generate novel changes to habitats and challenge local species survival (Williams et al. 2007; Amiel \& Shine 2012; IPCC 2014). Due to their extreme longevity, tuatara individuals that have hatched in the past decade could realistically live to experience increases in global mean air temperatures, which IPCC models predict will likely range from $0.3^{\circ} \mathrm{C}$ to $4.8^{\circ} \mathrm{C}$ by the 2100s (based on highest and lowest estimates from the CMIP5 model simulations RCP2.6 and RCP8.5; IPCC 2014). Increases toward the higher end of this range could pose a challenge to the tuatara's cold-adapted physiology. Ultimately, if the cognitive abilities of tuatara are plastic in response to environmental temperature, animals exposed to warmer environments under climate change may demonstrate enhanced learning ability. Improved cognitive ability and behavioural flexibility could enable individuals to respond effectively to novel situations and environmental stochasticity, and consequently have an increased chance of survival in unpredictable environments (Amiel \& Shine 2012). Spatial learning ability is likely to be a vital part of the cognitive repertoire of tuatara since they maintain home ranges, defend territories during the mating season, show burrow fidelity, and migrate to rookeries to nest (Gans et al. 1984; Newman 1987; Gillingham \& Miller 1991; Newman et al. 1994; Moore et al. 2009b; Cree 2014). Measuring spatial learning performance, and how this may be influenced by ambient temperature is therefore an ecologically relevant assessment of cognitive ability for tuatara. Here we aimed to determine whether juvenile tuatara tested on a spatial task at a higher ambient temperature were more likely to learn, or learn faster, than when tested at a lower ambient temperature.

\subsection{Methods}

\subsubsection{Study animals, husbandry and maintenance}

We sourced twenty juvenile tuatara from a captive-rearing facility in the Wellington region. Juveniles were hatched from eggs collected from Stephens Island/Takapourewa 
in the Cook Strait and incubated and hatched at Victoria University of Wellington in 2012 (Ethics approval number 2012R33 from the Victoria University of Wellington Animal Ethics Committee) until transfer to the captive-rearing facility where they were housed in secure, pest-free, outdoor enclosures under natural temperature, light and weather conditions. The sex of all juveniles had been determined using laparoscopy prior to transfer. Animals used in this study were transported to Victoria University in March 2015 for the duration of the study and were $\sim 3$ years old. Juveniles were kept in pairs in meshlidded metal enclosures $(690 \times 580 \times 320$ mm), lined with soil and leaf litter, and provided with several pieces of bark to serve as retreats. Juveniles were paired according to size and familiarity with others to prevent aggressive interactions between differently sized animals. Water was provided ad libitum in a plastic dish $(170 \times 110 \times 30 \mathrm{~mm})$ that was cleaned and refilled as needed. Juveniles were fed one medium cricket (Gryllus sp.) three times a week throughout the study. UV light was provided by Exo Terra ${ }^{\oplus} 20 \mathrm{~W}$ Repti Glo 5.0 UVB Tropical Terrarium lamps suspended above the enclosures and set to a 12:12 $\mathrm{h}$ light:dark cycle. Heat was not regulated and juveniles were maintained at ambient room temperature. Juveniles were housed in a room that had a constant airflow with the outside; therefore ambient room temperature likely fluctuated with outdoor temperature, but was buffered from extremes by adjacent rooms in the building. Outdoor mean daily temperatures for the March - September 2015 period were obtained from the nearest meteorological station (Kelburn Aws: $\left[-41.285^{\circ} \mathrm{S}, 174.768^{\circ} \mathrm{E}\right]$ ) using the NIWA (National Institute of Water and Atmospheric Research Ltd.) Cliflo database (http://www.cliflo.niwa.co.nz). Mean outdoor temperatures ranged from $8.9-16.8^{\circ} \mathrm{C}$. Juveniles were given three months (March-June 2015) to acclimatise to their enclosures before learning trials began. Original learning trials were run over 10 days in June 2015 (mean outdoor temp $=9.8^{\circ} \mathrm{C}$ ) and reversal-learning trials were run over 20 days in September 2015 (mean outdoor temp $=9.7^{\circ} \mathrm{C}$ ). All tuatara ate well and gained weight during the study. Juveniles were returned to the captive-rearing facility at the end of the study. 


\subsubsection{Experimental setup}

We tested spatial learning ability in a Y-maze using an anti-predator paradigm that had been used in previous reptile learning studies (Paulissen 2008; Amiel \& Shine 2012; Noble et al. 2012; Carazo et al. 2014). Juveniles were tested in identical Y-mazes (one per climate room/per temperature treatment) constructed from a plywood base with aluminium walls. Each arm measured $540 \times 100 \times 100 \mathrm{~mm}$ set at $120^{\circ}$ from each other with a central decision point (Figure 6.1). A clear section of Perspex was positioned atop each arm to prevent animals climbing out but enable observation. Two identical plastic retreats (170 $\times 170 \times 90 \mathrm{~mm}$ ) were placed at the ends of two arms, with the remaining arm used as the "start arm". Each retreat had a rectangular $(75 \times 50 \mathrm{~mm})$ hole cut to sit level with the floor of the maze and allow access into the retreat. Pieces of lightweight black plastic sheet were used to cover the access holes from within. One retreat served as the "accessible" retreat, and the other "inaccessible". The plastic sheet of the inaccessible retreat was fixed in place with tape and cardboard to prevent access while the curtain on the accessible retreat could be easily pushed through. Use of visually identical plastic sheets on both retreat entrances prevented visual discrimination between the two retreats during trials. Previous studies have used Plexiglas to prevent entry into refuges (Amiel \& Shine 2012); we did not use it in our study to avoid the possibility of the Plexiglas reflecting overhead lights and being visible to the juvenile tuatara. The positions of the accessible and inaccessible hides remained constant for each animal throughout trials but were alternated among individuals. Trials were recorded using a Nikon D3100 digital camera with a Nikon AF-S DX NIKKOR 18-55 mm f/3.5-5.6G VR camera lens positioned on a tripod and angled to face down on the maze. The tripod was consistently positioned between the rewarded and unrewarded arms of the maze. During trials the experimenter sat on a chair at the end of the start arm out of the tuatara's line of sight.

Whilst reptiles are capable of multiple learning mechanisms for performing successfully in spatial tasks, for the purposes of this study we were not concerned with the mechanism by which juveniles learned the location of the accessible refuge, but rather the impact that ambient temperature might have on this ability. As such, we did not control or limit the cues available to them and juveniles were able to utilise external visual cues (as the mazes remained in a constant position throughout trials animals could have 
used objects in the climate rooms e.g. camera and tripod, position of experimenter, shelving, lighting, etc.) and, though the arms of the Y-maze were not intentionally marked, juveniles may have responded to local cues within the maze that we were unaware of, such as texture, differences in colouration, and minor marks (Amiel et al. 2013).

\subsubsection{Learning trials}

Juvenile tuatara were divided into two temperature treatment groups. Ten animals were randomly assigned to the "low" temperature group $\left(15^{\circ} \mathrm{C}\right)$ and ten to the "high" temperature group $\left(25^{\circ} \mathrm{C}\right)$. Independent samples t-tests confirmed no significant differences between the mean mass ( $28 \pm 7 \mathrm{~g}$ and $32 \pm 7 \mathrm{~g}$ ) or mean snout-vent length (SVL; $107 \pm 6 \mathrm{~mm}$ and $105 \pm 9 \mathrm{~mm}$ ) of each treatment group (mass: $\mathrm{t}(18)=0.846, \mathrm{p}=$ 0.409; SVL: $\mathrm{t}(18)=0.676, \mathrm{p}=0.507)$. The experimental ambient temperatures were chosen as they fell within the active temperature range of tuatara and provided $a 10^{\circ} \mathrm{C}$ difference between treatment groups. An ambient temperature of $15^{\circ} \mathrm{C}$ was anticipated to result in lower activity levels in comparison to $25^{\circ} \mathrm{C}$, but juvenile tuatara were still expected to be active and able to exhibit a locomotive anti-predator response. All experimental trials were run in climate-controlled rooms at Victoria University of Wellington. Temperatures in both rooms were recorded every hour using dataloggers (Onset HOBO Pro v2 External Temperature/Relative Humidity Data Logger Part \#U23-002) and spot checked throughout trials using a handheld digital thermometer (RS 52 2063738). Prior to trials juveniles were transferred from their home pens into individual clear plastic containers $(290 \times 200 \times 135 \mathrm{~mm})$ lined with damp paper towel and provided with a refuge (160 $\mathrm{mm}$ plastic dish). These containers were then placed in the respective climate rooms. As there was variation in individual body size within treatment groups and larger body size has been shown to slow the rates of heating and cooling in ectotherms, we allowed all individuals one hour for acclimation to the experimental temperatures (Bartholomew \& Tucker 1964; McNab \& Auffenberg 1976; Stevenson 1985b).

At the beginning of each trial a juvenile was placed into the "start box", an area at the end of the start arm sectioned off by a removable cardboard wall (Figure 6.1). Each juvenile was given $60 \mathrm{~s}$ in the start box to settle after handling. The cardboard wall was then lifted and the juvenile was stimulated to move forward by brushing it on the tail with 
a fine paintbrush. Once the juvenile's hind legs had left the start box the trial began. Juveniles were allowed $180 \mathrm{~s}$ in the maze with their tails brushed from behind every $30 \mathrm{~s}$ to simulate a predatory attack and encourage them to seek refuge. Tail brushing was standardised at a maximum of five brushes or until the juvenile took two steps forward. A correct trial was scored if the juvenile entered only the correct arm of the maze (leading to the accessible refuge) and the accessible refuge within the trial time limit. Successful individuals were then allowed $180 \mathrm{~s}$ undisturbed inside the refuge to facilitate learning. A trial was scored as incorrect if the juvenile entered the incorrect arm (leading to the inaccessible refuge) at any point during the trial, didn't enter either arm, or failed to enter the accessible refuge within $180 \mathrm{~s}$. Entry into an arm or refuge was considered as entry of the head and both forelegs across the threshold. Unsuccessful individuals that were in the incorrect arm, central decision point or start arm after $180 \mathrm{~s}$ were caught by hand, placed outside the accessible refuge, coaxed inside (by nudging the base of the tail and hind legs) and allowed $180 \mathrm{~s}$ undisturbed. Juveniles that failed to enter the accessible refuge within $180 \mathrm{~s}$, but were in the correct arm were coaxed forward into the refuge and allowed 180 $\mathrm{s}$ undisturbed. After $180 \mathrm{~s}$ of undisturbed time the juvenile was returned to its plastic container. Half of the juveniles from each treatment (five from the $15^{\circ} \mathrm{C}$ and five from $25^{\circ} \mathrm{C}$ group) were trialled between 0900 and 1220 and the remaining half trialled between 1300 and 1640 each day. Each juvenile was tested twice a day for 10 consecutive days (i.e. 20 trials), with an intertrial interval of 100 minutes to allow for recovery. The precise time of day at which juveniles were tested was consistent throughout trials.

We recorded whether individuals had a correct/incorrect trial, latency to refuge entry (scored as $180 \mathrm{~s}$ if the juvenile did not enter the accessible refuge), and the number of incorrect turns made (i.e. if the juvenile entered the incorrect arm this was recorded as one incorrect turn). To determine if learning had occurred we followed a two-step protocol established by Carazo et al. (2014) in which, a) following at least four consecutively correct trials, the juvenile accumulated a significant number of correct trials according to a one-way binomial test (i.e. 5/5, 7/8, 9/11 correct and so on), and b) the juvenile maintained a significant number of correct trials from the first of the consecutively correct trials until the final trial (i.e. trial 20), also determined by a one-way binomial test. This second stage of categorisation was used to avoid labelling juveniles as 
learners when they may have attained a significant initial sequence of consecutively correct trials by chance.

\subsubsection{Memory trial}

The ability of juvenile tuatara to retain the learned location of the accessible refuge was assessed after a 10-day rest period. During this period juveniles were not exposed to the Y-maze and remained in their home pens. Their feeding regime was not altered. During the memory trial each juvenile was run once in the Y-maze following the same protocol and at the same temperature and time as during the learning trials. We recorded whether a juvenile had a correct/incorrect trial, latency to refuge entry and the number of incorrect turns made.

\subsubsection{Reversal learning trials}

To assess learning ability in each juvenile tuatara at both experimental temperatures we employed a reversal-learning paradigm in which juveniles were tested in the alternate temperature treatment (i.e. those previously assessed at $25^{\circ} \mathrm{C}$ were then assessed at $15^{\circ} \mathrm{C}$ ) and the positions of the accessible and inaccessible refuges were switched (i.e. if the accessible refuge was at the end of the right arm during learning trials, it was moved to the end of the left). Although juveniles were transferred to the alternate temperature treatment and positions of the accessible and inaccessible refuges were switched, juveniles were tested in the same maze and climate room as before so that familiar extra and intra-maze cues were available for navigation. Juveniles were given a 64-day rest period between the memory trial and the first 

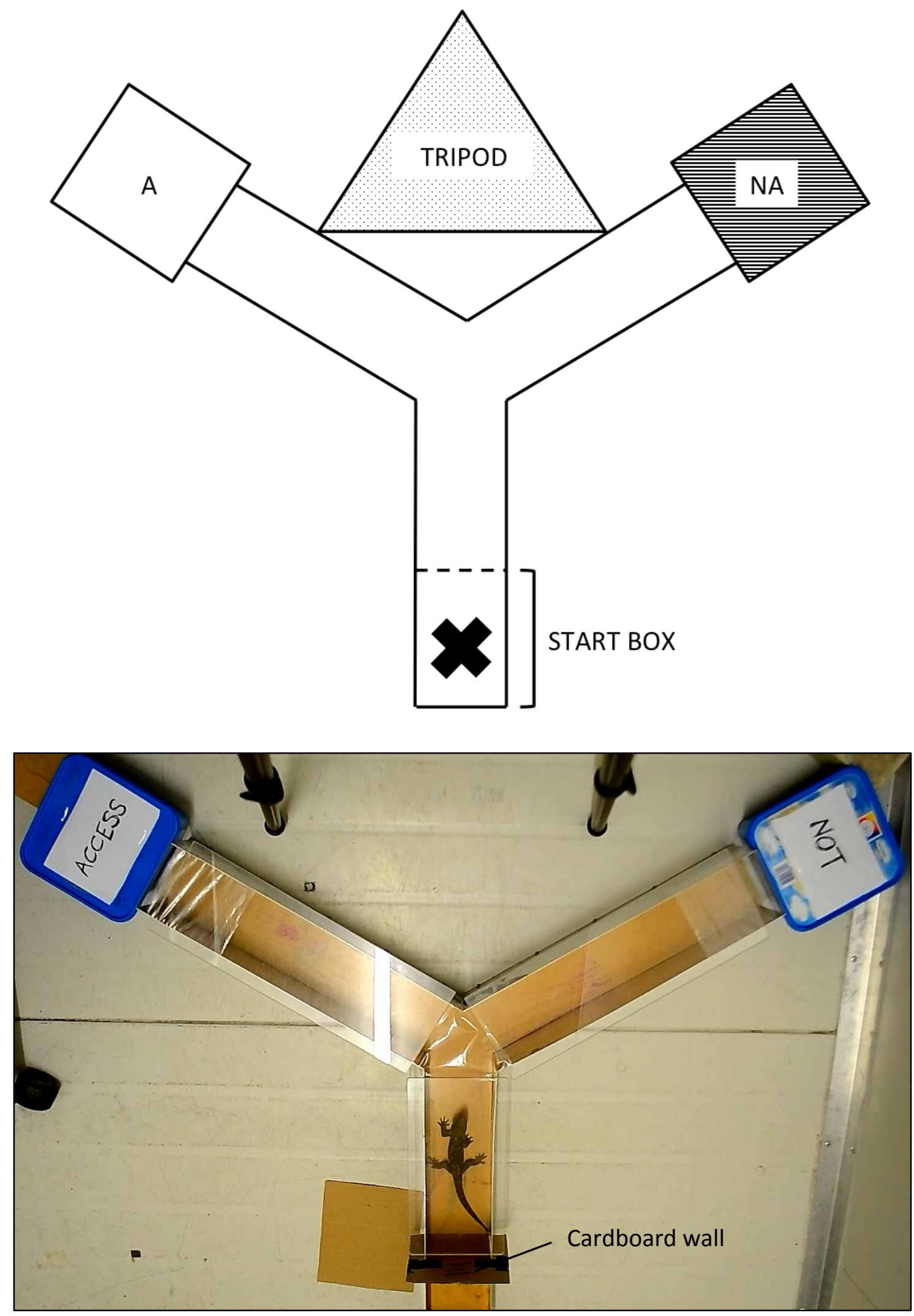

Figure 6.1 Top image: Diagram of Y-maze experimental setup showing location of maze, hides, tripod and camera. " $\mathrm{A}$ " = accessible refuge and "NA" = non-accessible refuge. Dashed line indicates position of temporary cardboard wall in start arm and boundary of start box. " $X$ " indicates point at which juveniles were entered into the maze. Bottom image: Still image of Y-maze setup with a juvenile tuatara outside of the start box in the start arm. 
reversal-learning trial. Reversal-learning trials followed the same protocols as the learning trials and memory trial. However, the number of trials per juvenile was doubled to 40 , as we expected prior learning of the accessible refuge location to increase the number of trials required to reverse this association and learn the new location. We recorded whether a juvenile had a correct/incorrect trial, latency to refuge entry and the number of incorrect turns made. Learning was established using the same criteria as employed for the learning trials, with the exception that juveniles had to maintain a significant number of correct trials from the first of the consecutively correct trials until trial 40.

\subsubsection{Statistical analyses}

All analyses were performed in R v. 3.2.2 ( $R$ Core Team 2015). We analysed the relationships between learning performance in the first spatial task and multiple variables using generalised linear models (GLMs) with a binomial distribution. The package "Ime4" was used to perform generalised linear analyses (Bates et al. 2016). We categorised individuals as "learner" or "non-learner" and modelled this outcome as a function of temperature treatment (i.e. $25^{\circ} \mathrm{C}$ or $15^{\circ} \mathrm{C}$ ), sex, mass, and snout-vent length (SVL). Latencies of learners to enter the accessible hide and number of incorrect turns made were assessed as functions of individual, temperature treatment, sex, trial number and mass using generalized linear mixed models (GLMMs) with a binomial distribution (for correct or incorrect turn) or Poisson distribution (for latency data) and individual ID as a random factor. We also analysed rate of learning within learners by modelling acquisition trial (the trial in which, following four consecutively correct trials, a juvenile accumulated a significant number of correct trials according to a one-way binomial test) as a function of temperature, SVL, mass, and sex using GLMs with a Poisson distribution. Following the reversal-learning task, we analysed performance of individuals using GLMs with a binomial distribution. Learning outcomes (learned or did not learn) were modelled as functions of temperature treatment, sex, mass, and SVL. As the parameter to sample size ratio was consistently less than $40(n / K<40)$, the suitability of models was compared using the second-order Akaike information criterion $\left(A I C_{C}\right)$ value for small samples, obtained using the R package "AICcmodavg" (Burnham \& Anderson 2003; Mazerolle 2016). The best-fit model was then compared to the null model using the chi-squared statistic, which, 
if significant, indicated that the inclusion of predictors significantly improved the fit of the model. We did not include both measures of body size (SVL and mass) in the same model to avoid autocorrelation. Considering our small sample size we restricted the number of predictor variables to one per 10 data points, so for a sample size of 20 we included a maximum of two predictors in each model. GLMMs were only used when we had enough replicates per individual to include individual ID as a random factor i.e. when looking at latencies of learners per trial.

\subsection{Results}

\subsubsection{Learning trials: Learners}

After 20 trials $40 \%(8 / 20)$ of individuals had learned the location of the accessible hide in the Y-maze, taking between 5 and 19 trials (mean $=11 \pm 1.66$ ) to do so. Sixty per cent $(6 / 10)$ of the $25^{\circ} \mathrm{C}$ temperature treatment group were categorised as learners compared to $20 \%(2 / 10)$ of the $15^{\circ} \mathrm{C}$ treatment group. A third individual from the $15^{\circ} \mathrm{C}$ group accumulated a significant first tally, but this did not remain significant until trial 20 . The GLM that provided the best fit to our data $(\chi 2=15.71, p<0.001$, Table 6.1$)$ showed a trend for temperature treatment to affect learning, with a greater number of juveniles in the $25^{\circ} \mathrm{C}$ group learning the task (estimate $=5.89 \pm 3.04, z=1.93, p=0.053$ ) and $a$ significant effect of SVL (estimate $=-0.43 \pm 0.21, z=-2.06, p=0.039$ ), with smaller juveniles more likely to learn the location of the accessible refuge (Figure 6.2). An interaction term between SVL and temperature treatment was not included in the model due to the sample size $(n=20)$. Temperature was found to be the most significant predictor of acquisition trial $(\chi 2=5.243, p=0.022)$, with the two juveniles in the $15^{\circ} \mathrm{C}$ treatment group acquiring the task sooner (estimate $=0.6405 \pm 0.301, z=2.130, p=0.033$, Figure 6.3). However, given the comparatively low weight of the model and considering that the two learners from the $15^{\circ} \mathrm{C}$ group were also the smallest learners, temperature and SVL are confounded and it is possible that variation in the rate of learning was influenced by body size (Table 6.2, Figure 6.3). 
Table 6.1. Results of analysis of competing GLMs on factors influencing learning. $\mathrm{K}=$ number of estimated parameters in the model. $A I C_{C}=$ Akaike information criterion accounting for small sample size. $\Delta\left(A I C_{C}\right)=\left[A I C_{C}-\min \left(A I C_{C}\right)\right]$. Relative likelihood $=\exp \left(-0.5^{*} \Delta\left(A I C_{C}\right)\right) \cdot w\left(A I C_{C}\right)=$ weight of the model.

\begin{tabular}{|c|c|c|c|c|c|}
\hline Model predictor variables & K & $\mathrm{AlC}_{\mathrm{c}}$ & $\Delta\left(A \mid C_{C}\right)$ & $\begin{array}{c}\text { Relative } \\
\text { likelihood }\end{array}$ & $w\left(A I C_{C}\right)$ \\
\hline SVL + Temperature & 3 & 18.710 & 0 & 1 & 0.831 \\
\hline Mass + Temperature & 3 & 22.687 & 3.977 & 0.137 & 0.114 \\
\hline SVL & 2 & 25.505 & 6.795 & 0.033 & 0.028 \\
\hline Mass & 2 & 27.256 & 8.547 & 0.014 & 0.012 \\
\hline$S V L+\operatorname{Sex}$ & 3 & 28.258 & 9.548 & 0.008 & 0.007 \\
\hline Temperature & 2 & 28.174 & 9.465 & 0.009 & 0.007 \\
\hline Null model & 1 & 29.143 & - & - & - \\
\hline Sex & 2 & 31.590 & 12.880 & 0.002 & 0.001 \\
\hline
\end{tabular}

Table 6.2. Results of analysis of competing GLMs on factors influencing acquisition trial number. $K=$ number of estimated parameters in the model. $\mathrm{AIC}_{\mathrm{C}}=$ Akaike information criterion accounting for small sample size. $\Delta\left(\mathrm{AIC}_{\mathrm{C}}\right)=\left[\mathrm{AIC}_{\mathrm{C}}\right.$ $\left.-\min \left(A I C_{C}\right)\right]$. Relative likelihood $=\exp \left(-0.5^{*} \Delta\left(A I C_{C}\right)\right) \cdot w\left(A I C_{C}\right)=$ weight of the model.

\begin{tabular}{lrrrrr}
\hline \multicolumn{1}{c}{ Model predictor variables } & K & \multicolumn{1}{c}{ AIC } & $\Delta\left(\right.$ AIC $\left._{\mathrm{c}}\right)$ & $\begin{array}{r}\text { Relative } \\
\text { likelihood }\end{array}$ & w(AIC $)$ \\
\hline Temperature & $\mathbf{2}$ & $\mathbf{4 8 . 2 4 0}$ & $\mathbf{0}$ & $\mathbf{1}$ & $\mathbf{0 . 4 9 6}$ \\
SVL & 2 & 49.505 & 1.264 & 0.531 & 0.264 \\
Null model & 1 & 49.750 & - & - & - \\
Mass & 2 & 52.273 & 4.032 & 0.133 & 0.066 \\
Sex & 2 & 52.828 & 4.588 & 0.101 & 0.050 \\
Temperature + Sex & 3 & 52.622 & 4.382 & 0.112 & 0.055 \\
Temperature + Mass & 3 & 53.551 & 5.311 & 0.070 & 0.035 \\
Temperature + SVL & 3 & 53.605 & 5.365 & 0.068 & 0.034 \\
\hline
\end{tabular}




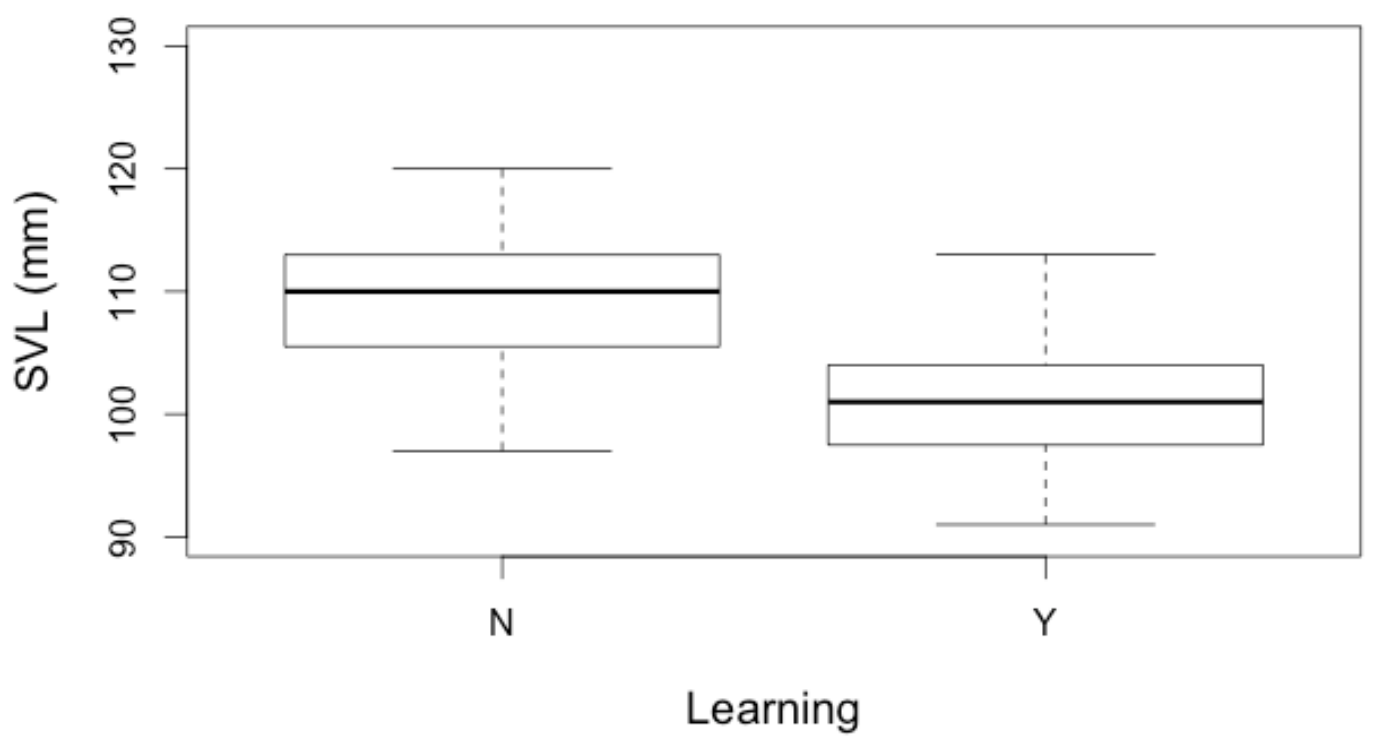

Figure 6.2 Boxplot of mean SVL ( $\mathrm{mm}$ ) of learners $(Y)$ and non-learners $(N)$. Learners $(n=8)$ had a mean SVL of 101.13 $\mathrm{mm}$ while non-learners $(n=12)$ had a mean SVL of $109.25 \mathrm{~mm}$. Boxplots show the median and interquartile range and whiskers extend up to 1.5 times the interquartile range.

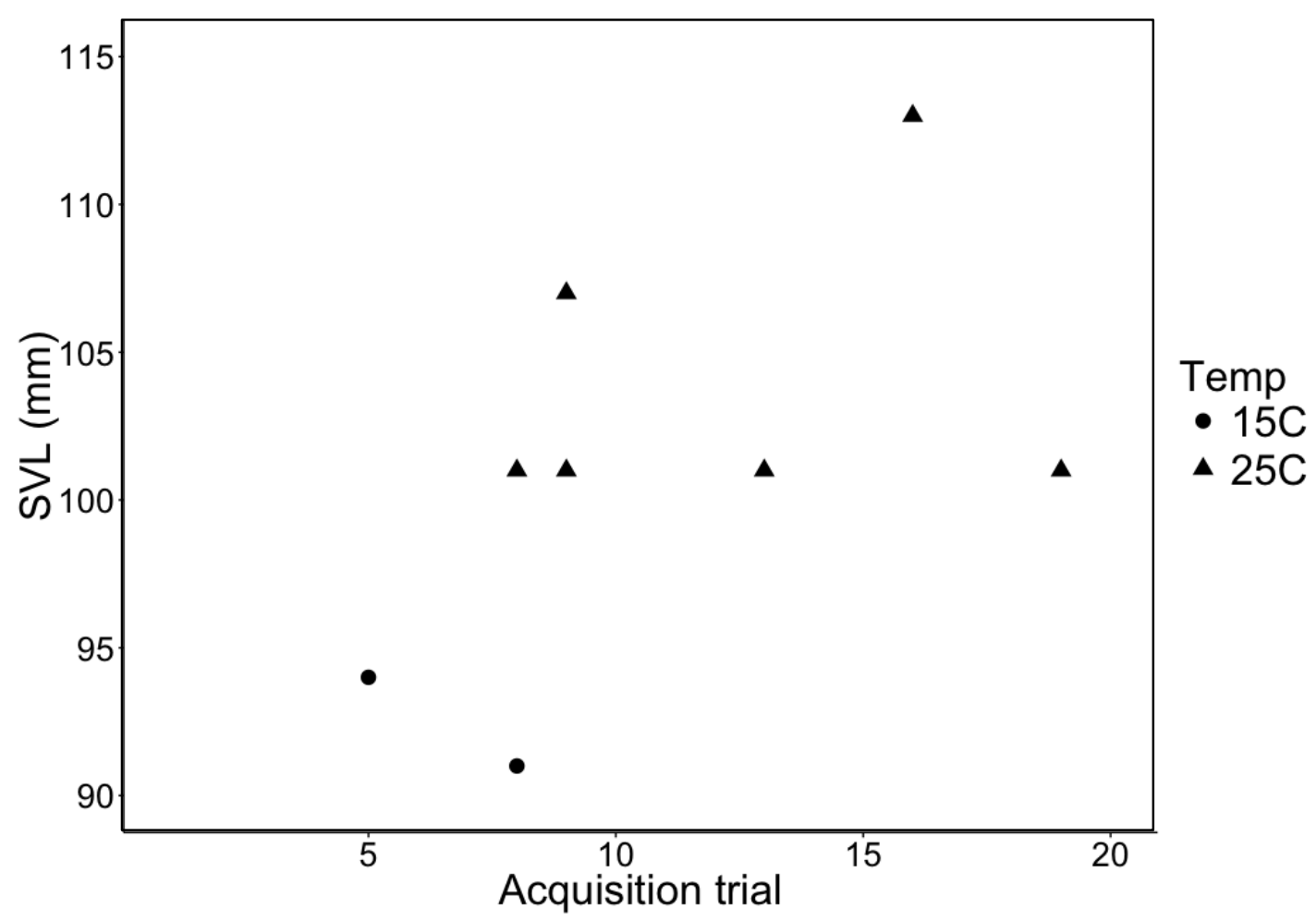

Figure 6.3 Scatterplot showing learner acquisition trial, temperature treatment group and SVL $(\mathrm{mm})$ measurement. The smallest learners were also those in the $15^{\circ} \mathrm{C}$ treatment group, and learned the location of the accessible hide faster than larger individuals in the $25^{\circ} \mathrm{C}$ treatment group. 


\subsubsection{Learning trials: Latency and incorrect turns}

Individual mean latencies to enter the accessible hide ranged from 19.26-79.00 seconds. Mean latency to enter the accessible hide over all 20 trials was $57.11 \pm 32.87 \mathrm{~s}$ for the six learners in the $25^{\circ} \mathrm{C}$ group and $36.47 \pm 25.38 \mathrm{~s}$ for the two learners in the $15^{\circ} \mathrm{C}$ group. The GLMM that best explained variation in latency among the eight learners $(\chi 2=46.98, p<$ 0.001) showed a significant effect of individual ID on latency, with some individuals significantly faster to enter the accessible hide than others (Table 6.3). The GLMM that best explained differences in the number of incorrect turns made among learners $(x 2=$ 15.678, $p<0.001$ ) showed a significant effect of trial number (estimate $=-0.16571 \pm$ $0.04634, z=-3.576, p<0.001$ ), with the number of incorrect turns made by learners decreasing as expected with successive trials (Table 6.4).

Table 6.3 Results of analysis of competing GLMMs on factors affecting latency to enter the accessible hide. $\mathrm{K}=$ number of estimated parameters in the model. $\mathrm{AIC}_{\mathrm{C}}=$ Akaike information criterion accounting for small sample size. $\Delta\left(\mathrm{AIC}_{\mathrm{C}}\right)=$ $\left[A I C_{C}-\min \left(A I C_{C}\right)\right]$. Relative likelihood $=\exp \left(-0.5^{*} \Delta\left(A I C_{C}\right)\right) \cdot w\left(A I C_{C}\right)=$ weight of model.

\begin{tabular}{lrcrrr}
\hline Model predictor variables & K & \multicolumn{1}{c}{ AIC $_{\mathbf{c}}$} & \multicolumn{1}{c}{$\boldsymbol{\Delta}\left(\mathrm{AIC}_{\mathrm{C}}\right)$} & $\begin{array}{c}\text { Relative } \\
\text { likelihood }\end{array}$ & \multicolumn{1}{c}{$\mathbf{w}_{(\mathrm{AIC})}$} \\
\hline Individual ID & $\mathbf{9}$ & $\mathbf{2 4 3 2 . 1 0 1}$ & $\mathbf{0}$ & $\mathbf{1}$ & $\mathbf{0 . 7 5 1}$ \\
Trial no. & 3 & 2434.305 & 2.204 & 0.332 & 0.249 \\
Temperature treatment & 3 & 2462.020 & 29.919 & 0.000 & 0.000 \\
Sex & 3 & 2463.364 & 31.263 & 0.000 & 0.000 \\
Null model & 2 & 2463.695 & - & - & - \\
Time of day & 3 & 2464.509 & 32.408 & 0.000 & 0.000 \\
Mass & 3 & 2464.789 & 32.688 & 0.000 & 0.000 \\
\hline
\end{tabular}

Table 6.4 Results of analysis of competing GLMMs on factors affecting number of incorrect turns made. $\mathrm{K}=$ number of estimated parameters in the model. $\mathrm{AIC}_{\mathrm{C}}=$ Akaike information criterion accounting for small sample size. $\Delta\left(\mathrm{AIC}_{\mathrm{C}}\right)=\left[\mathrm{AIC}_{\mathrm{C}}\right.$ $\left.-\min \left(A I C_{C}\right)\right]$. Relative likelihood $=\exp \left(-0.5^{*} \Delta\left(A I C_{C}\right)\right) \cdot w\left(A I C_{C}\right)=$ weight of model.

\begin{tabular}{|c|c|c|c|c|c|}
\hline Model predictor variables & K & $\mathrm{AlC}_{\mathrm{c}}$ & $\Delta\left(\mathrm{AIC}_{\mathrm{C}}\right)$ & $\begin{array}{c}\text { Relative } \\
\text { likelihood }\end{array}$ & $w\left(A I C_{c}\right)$ \\
\hline Trial no. & 3 & 131.431 & 0 & 1 & 0.990 \\
\hline Temperature treatment & 3 & 141.684 & 10.253 & 0.006 & 0.006 \\
\hline Sex & 3 & 142.892 & 11.461 & 0.003 & 0.003 \\
\hline Null model & 2 & 145.031 & - & - & - \\
\hline Mass & 3 & 146.136 & 14.705 & 0.001 & 0.001 \\
\hline Time of day & 3 & 146.756 & 15.325 & 0.001 & 0.001 \\
\hline Individual ID & 9 & 148.380 & 16.949 & 0.000 & 0.000 \\
\hline
\end{tabular}




\subsubsection{Memory}

All eight individuals that had learned the location of the accessible hide during the learning trials performed correctly on the single memory trial after a 10-day break. Six (out of twelve) individuals that were not categorised as learners also performed correctly on the memory task. One of these individuals had achieved a significant first tally during learning trials, with acquisition in trial 17 , but did not maintain a significant final tally by trial 20.

\subsubsection{Reversal learning}

After 40 trials, $15 \%(3 / 20)$ of individuals had learned the location of the accessible refuge in the Y-maze. Juveniles took between 14 and 36 (mean $=23 \pm 6.57$ ) trials to learn the reversed location (Table 6.5). Twenty per cent $(2 / 10)$ of the $25^{\circ} \mathrm{C}$ temperature treatment group were categorised as learners compared to $10 \%(1 / 10)$ of the $15^{\circ} \mathrm{C}$ treatment group (Table 6.5). The GLM that provided the best fit to the reversal learning data was not an improvement over the null model $(\chi 2=1.482, p=0.224$, Table 6.6$)$, indicating that the inclusion of this predictor did not improve the model's predictive value. Taken with the similar AIC values and low weight of the top ranked and other models and, this suggests that, as only three individuals learned the reversal task, this sample size is too small for any meaningful statistical analysis of factors influencing reversal-learning ability. The three individuals that learned had similar mean weights and SVL measurements (mass: $35.27 \pm 7.51 \mathrm{~g}$; SVL: $107.33 \pm 9.07 \mathrm{~mm}$ ) to those that did not learn (mass: $35.61 \pm 6.91 \mathrm{~g}$; SVL: $107.82 \pm 7.26 \mathrm{~mm}$ ), suggesting no influence of body size on learning of the reversal task. Similarly, among the three learners, there appeared to be no influence of body size on acquisition trial or latency to enter the accessible hide (Table 6.5). One of the six individuals that learned the original location of the refuge in the $25^{\circ} \mathrm{C}$ treatment group was able to learn the location of the hide during the reversal task at $15^{\circ} \mathrm{C}$; the remaining five individuals showed no evidence of learning. Of the two individuals that learned the original task at $15^{\circ} \mathrm{C}$, one of these learned the reversal task at $25^{\circ} \mathrm{C}$ while the other, though it did accumulate a significant first tally, did not achieve a significant final tally and was 
classed as a non-learner. The other individual that learned the reversal task at $25^{\circ} \mathrm{C}$ had not learned the original task at $15^{\circ} \mathrm{C}$ (Table 6.7).

Table 6.5 Comparisons of individuals that learned the reversal task. No apparent trend for mass or SVL to influence rate of learning (acquisition trial) or mean latency to enter the accessible hide.

\begin{tabular}{llrrrrr}
\hline Individual & Sex & $\begin{array}{c}\text { Treatment } \\
\left({ }^{\circ} \mathbf{C}\right)\end{array}$ & Mass (g) & SVL (mm) & $\begin{array}{c}\text { Acquisition } \\
\text { trial }\end{array}$ & $\begin{array}{c}\text { Mean } \\
\text { latency (s) }\end{array}$ \\
\hline $5(34) 53$ & $\mathrm{M}$ & 15 & 40.01 & 114 & 36 & 66.50 \\
5544 & $\mathrm{~F}$ & 25 & 39.19 & 111 & 14 & 89.04 \\
5554 & $\mathrm{M}$ & 25 & 26.62 & 97 & 20 & 48.61 \\
\hline
\end{tabular}

Table 6.6 Results of analysis of competing GLMs on factors affecting learning of the reversal task. $K=$ number of estimated parameters in the model. $\mathrm{AIC}_{\mathrm{C}}=$ Akaike information criterion accounting for small sample size. $\Delta\left(\mathrm{AIC}_{\mathrm{C}}\right)=\left[\mathrm{AIC}_{\mathrm{C}}\right.$ $\left.-\min \left(A I C_{C}\right)\right]$. Relative likelihood $=\exp \left(-0.5^{*} \Delta\left(A I C_{C}\right)\right) \cdot w\left(A I C_{C}\right)=$ weight of model.

\begin{tabular}{|c|c|c|c|c|c|}
\hline Model predictor variables & K & $\mathrm{AlC}_{\mathrm{C}}$ & $\Delta\left(A \mid C_{C}\right)$ & $\begin{array}{c}\text { Relative } \\
\text { likelihood }\end{array}$ & $w\left(A I C_{C}\right)$ \\
\hline Null model & 1 & 19.131 & - & - & - \\
\hline Sex & 2 & 20.133 & 0 & 1 & 0.321 \\
\hline Temperature & 2 & 21.216 & 1.083 & 0.582 & 0.187 \\
\hline SVL & 2 & 21.602 & 1.470 & 0.480 & 0.154 \\
\hline Mass & 2 & 21.608 & 1.475 & 0.478 & 0.154 \\
\hline Temperature + Sex & 3 & 22.653 & 2.520 & 0.284 & 0.091 \\
\hline Temperature + SVL & 3 & 24.009 & 3.877 & 0.144 & 0.046 \\
\hline Temperature + Mass & 3 & 24.009 & 3.877 & 0.144 & 0.046 \\
\hline
\end{tabular}


Table 6.7 Performance of individuals in learning and reversal learning tasks, as well as individual sex and morphometric measurements at the first learning trials. A " $\checkmark$ " (tick) denotes an individual that learned the task. A " $\sim$ " denotes an individual that achieved a significant first tally, but not a significant final tally and was not classed as a learner.

\begin{tabular}{cllrrrr}
\hline Treatment group & $\begin{array}{c}\text { Tuatara } \\
\text { ID }\end{array}$ & Sex & \multicolumn{2}{c}{$\begin{array}{c}\text { Starting } \\
\text { measurements }\end{array}$} & \multicolumn{2}{c}{ Temperature } \\
& & & Mass (g) & SVL (mm) & $\mathbf{2 5}^{\circ} \mathbf{C}$ & $\mathbf{1 5}^{\circ} \mathbf{C}$ \\
\hline & 5243 & $\mathrm{~F}$ & 31.35 & 101 & $\checkmark$ & \\
& 5545 & $\mathrm{~F}$ & 28.44 & 101 & $\checkmark$ & \\
& 5542 & $\mathrm{~F}$ & 30.69 & 101 & $\checkmark$ & \\
Learning $25^{\circ} \mathrm{C} \rightarrow$ & 5325 & $\mathrm{M}$ & 35.69 & 114 & & \\
Reversal $15^{\circ} \mathrm{C}$ & 5323 & $\mathrm{~F}$ & 36.05 & 107 & $\checkmark$ & \\
& 5333 & $\mathrm{M}$ & 34.26 & 107 & & \\
& 4525 & $\mathrm{~F}$ & 49.58 & 118 & & \\
& 4452 & $\mathrm{~F}$ & 35.21 & 109 & & \\
& 5445 & $\mathrm{M}$ & 25.49 & 101 & $\checkmark$ & \\
& $5(34) 53$ & $\mathrm{M}$ & 39.57 & 113 & $\checkmark$ & $\checkmark$ \\
\hline & & & & & $\mathbf{1 5}{ }^{\circ} \mathrm{C}$ & $\mathbf{2 5 ^ { \circ } \mathrm { C }}$ \\
\hline & 5535 & $\mathrm{M}$ & 34.30 & 111 & $\sim$ & \\
& 4532 & $\mathrm{~F}$ & 25.62 & 97 & & \\
& 4542 & $\mathrm{M}$ & 24.69 & 99 & & \\
Learning $15^{\circ} \mathrm{C} \rightarrow$ & 5544 & $\mathrm{~F}$ & 36.61 & 109 & & $\checkmark$ \\
Reversal $25^{\circ} \mathrm{C}$ & 5554 & $\mathrm{M}$ & 23.34 & 94 & $\checkmark$ & $\checkmark$ \\
& 4524 & $\mathrm{~F}$ & 38.60 & 112 & & \\
& 4545 & $\mathrm{M}$ & 21.60 & 91 & $\checkmark$ & $\sim$ \\
& 4454 & $\mathrm{~F}$ & 42.75 & 120 & & \\
& 5434 & $\mathrm{~F}$ & 36.85 & 111 & & \\
& 5255 & $\mathrm{~F}$ & 35.27 & 104 & & \\
\end{tabular}

\subsection{Discussion}

I investigated the ability of juvenile tuatara to acquire and retain a novel operant learning task at two different ambient temperatures, to determine whether warmer test air temperatures may affect cognitive abilities in this cold-adapted reptile. While we did not assess the precise cues used by juvenile tuatara to navigate in the $\mathrm{Y}$-maze, our results demonstrate that tuatara are capable of spatial learning and add to previous work by Northcutt and Heath (1973) and Woo et al. (2009), which showed that tuatara can perform successfully on spatial and associative learning tasks. Our results also provide further evidence that, despite previous misconceptions, reptiles can be quick learners, particularly when tasks are designed to accommodate natural behaviours and ecologically relevant stimuli (Burghardt 2013). Our results suggest a trend for tuatara to display enhanced leaning ability when trained at a warmer ambient temperature of $25^{\circ} \mathrm{C}$ than 
when trained at a cooler ambient temperature of $15^{\circ} \mathrm{C}$. Overall, eight juveniles were able to learn the accessible hide's location when trialled in the warmer treatment, compared to only three individuals when trialled at the cooler temperature. Reversal learning ability was poor in both experimental conditions, with five of the six animals that learned the original refuge location at $25^{\circ} \mathrm{C}$ unable to learn the reversed location at $15^{\circ} \mathrm{C}$. Reversal learning ability at $25^{\circ} \mathrm{C}(\mathrm{n}=2)$ was similar to learning ability at $15^{\circ} \mathrm{C}(\mathrm{n}=1)$.

Studies on incubation temperature and reptile cognition suggest that incubation temperature might influence the development and morphology of brain regions that control learning (Amiel \& Shine 2012; Clark et al. 2013). Whilst temperature-modified brain morphology is an unlikely explanation for our results, ambient temperature has been shown to influence neural activity and brain function in the mammalian hippocampus, a homologue of the reptilian medial and dorsal cortices (Schiff \& Somjen 1985; Moser et al. 1993; Andersen \& Moser 1995; Shibasaki et al. 2007; de la Peña et al. 2012). Similarly, studies by Andry et al. (1971) and Borsook et al. (1978) on other ectothermic species also suggest that altered neural activity may play a role, though the mechanisms by which ambient temperature may affect neural activity and learning ability remain unclear (Krekorian et al. 1968; Moser et al. 1993; Day et al. 2001; López et al. 2003; Wilkinson \& Huber 2012). Conversely, a review of heat stress and cognitive performance in humans discusses the Maximal Adaptability Model, which asserts that temperature affects performance through competition for attentional resources (Kahneman 1973; Hancock 1989; Hancock \& Vasmatzidis 2003). This model suggests that optimal cognitive performance occurs at temperatures within the individual's preferred optimal temperature zone (where environmental stress is minimal) and performance declines as environmental stress increases and attentional resources are depleted (Hancock \& Vasmatzidis 2003). Heat stress can affect cognitive performance by significantly damaging thermo-sensitive neural circuits and impairing neural performance (Robertson 2004a, 2004b). This can also lead to impaired behaviour and motor function and the extreme, exposure to high temperatures can cause permanent impairment of function or death of the organism (Robertson 2004a). Whilst this model is discussed in regard to human cognition, the concept that more of an individual's attentional resources might be allocated to a task when trained at temperatures that evoke minimal stress (i.e. around the organism's preferred temperature) provides interesting context for the difference in 
learning ability observed in tuatara trained at $15^{\circ} \mathrm{C}$ and $25^{\circ} \mathrm{C}$. While an ambient temperature of $15^{\circ} \mathrm{C}$ falls well within the tuatara's active range and should not cause thermal stress, the preferred body temperature of juvenile tuatara is $21.3^{\circ} \mathrm{C}$ (with a range of $19.3-24.9^{\circ} \mathrm{C}$; Besson \& Cree 2010), thus an experimental temperature of $25^{\circ} \mathrm{C}$ likely lies at the upper range of their preferred optimal temperature zone and could suggest that individuals were better learners at this higher temperature because they were able to allocate more attention to the learning task.

It is important to acknowledge limitations of the two temperature treatments used. Treatment temperatures of $15^{\circ} \mathrm{C}$ and $25^{\circ} \mathrm{C}$ were selected as they fall safely within the active temperature range of tuatara whilst allowing for a $10^{\circ} \mathrm{C}$ difference between treatment groups. The $25^{\circ} \mathrm{C}$ treatment used is likely to be ecologically relevant in the future, as it is representative of the mean temperatures that several sites supporting tuatara populations may experience following the maximum predicted $4.8^{\circ} \mathrm{C}$ air temperature increase (IPCC 2014). However, whilst there may be initial enhancement of learning ability for tuatara as the climate warms, as climate change progresses temperatures will continue to rise and we are unable to say if temperatures beyond $25^{\circ} \mathrm{C}$ will see additional cognitive benefits or deficits as temperatures approach the species' upper limits, as would also be expected under the Maximal Adaptability Model, though it could be argued that deficits would be more likely given the potential for heat stress, which could impact neural function, and that captive air temperatures above $25^{\circ} \mathrm{C}$ have been associated with tuatara fatality (Blanchard 2002). It would consequently be useful to assess learning ability at temperatures above $25^{\circ} \mathrm{C}$, though there could be physiological risks associated with maintaining tuatara for several hours at temperatures close to the upper limit of their active temperature range and close to their panting threshold $\left(31^{\circ} \mathrm{C}\right)$ and factors such as humidity/water loss might also influence behaviour (Heatwole 1982; Corkery et al. 2014a).

Analysis of the original learning task showed that body size also affected both learning ability and possibly rate of learning, though played no role in locomotor speed (measured as latency to enter the accessible hide) in the y-maze. Animals with smaller SVL measurements were more likely to learn the location of the accessible hide and, within those classed as "learners", smaller animals may have been faster to learn the location, though confounding with temperature treatment make this relationship 
uncertain. These relationships were not observed in learners and non-learners of the reversal task. That smaller individuals were both better and potentially faster learners could be attributed to differences in past experience of differently sized conspecifics and motivation to escape the predatory stimulus (Rowe \& Healy 2014). Prior to this study, juvenile tuatara were housed with multiple conspecifics in semi-natural enclosures. It is feasible that competitive/aggressive interactions over food or territory acquisition affected the behavioural responses and body sizes of juveniles, enhancing the motivation of consequently smaller juveniles (that likely lost in competitive interactions) to seek shelter when presented with a threatening stimulus and so escape from potentially costly exchanges. Whilst differing degrees of motivation have not been explicitly addressed in other studies that stimulated an anti-predator response, a study by Carazo et al. (2014) on adult eastern water skinks (Eulamprus quoyii) found a link between boldness and learning ability, with behaviourally "shy" and "bold" animals shown to be better learners than intermediate animals, indicating that an individual's behavioural type can influence its spatial learning ability, or vice versa (Carazo et al. 2014). Alternatively, as predation can be size-dependent, with small, weak animals often more vulnerable, it follows that smaller, comparatively poorer condition juveniles might display a comparatively stronger antipredator response and actively seek shelter following a simulated predatory attack, causing them to be both more likely and faster to learn the location of a refuge (Stein \& Magnuson 1976; Werner 1986; Alexander \& Covich 1991; Gliwicz \& Umana 1994; Memmott et al. 2000; McLennan et al. 2004).

More individuals learned the original spatial task (8/20) than the reversal task $(3 / 20)$ and it is important to note that while $45 \%$ of juveniles were able to learn the accessible refuge location during either the original or reversal task, only two of these animals showed learning on both. While this demonstrates that these two individuals were capable of behavioural flexibility, it also suggests that the reversal task may have been too challenging, or that juveniles may have needed more than the 40 trials allowed to learn the new refuge location (Stone et al. 2000; Shettleworth 2010; Lucon-Xiccato \& Bisazza 2014). When studying learning the suitability of the reinforcer must be carefully considered, as the reinforcer and schedule of reinforcement may affect acquisition of the reinforced stimulus. Although provision of a positive reward (e.g. food) for accessing the hide would likely have been motivational, we attempted to maximise learning by using 
shelter from a negative stimulus (i.e. "predator") as a highly ecologically relevant reinforcer, which had been successfully used in several studies on reptiles (Northcutt \& Heath 1973; Paulissen 2008; Amiel \& Shine 2012; Noble et al. 2012; Carazo et al. 2014). While a threatening stimulus should be more immediately motivating than the prospect of a heat or food reward (as it poses a more immediate threat to survival), it is also possible that habituation to the predatory stimulus (i.e. observer and paint brush) over time could cause a reduction in the strength of the reinforcer, which could explain the poorer overall performance in the reversal learning trials (Carazo et al. 2014). However, that most juveniles consistently moved in response to the stimulus and repeatedly attempted to escape the maze/enter hides suggests that the predatory stimulus was sufficiently motivating, but juveniles were unable to learn the reversal.

Prior experience in an environment improves spatial learning ability in lizards (Paulissen 2008). However anthropogenic climate change has the potential to generate novel habitats and challenges, thus we sought to replicate novel environmental change by testing individuals in an environment with which they had no prior experience. Fortyfive per cent of juveniles learned the location of the accessible refuge in the original and/or reversal task; this low rate of acquisition could be attributable to a lack of experience in the learning environment. However, this rate is comparable to results from similar experiments: $32-34 \%$ of Eastern water skinks (E. quoyii) were categorized as learners in two different spatial learning studies and $50 \%$ of juvenile spotted pythons $(A$. maculosus) were able to learn the location of a shelter in an arena (Stone et al. 2000; Noble et al. 2012; Carazo et al. 2014). Our results also contrast with previous findings from a study on little brown skinks (Scincella lateralis), which found that animals were incapable of spatial learning without prior experience in the test arena (Paulissen 2008). In contrast, the juvenile tuatara tested in this study were able to learn the location of a refuge in a novel Y-maze, in some cases after only a few trials, without any prior experience in the test environment. Whilst there are limitations to comparing results from studies with different methodologies, spatial tasks and learning criteria, overall, juvenile tuatara show similar acquisition rates to adult tuatara, juvenile corn snakes (Elaphe guttata guttata), caiman (Caiman sclerops), and eastern water skinks (E. quoyii)(Northcutt \& Heath 1971, 1973; Holtzman et al. 1999; Noble et al. 2012; Carazo et al. 2014). Acquisition rates were also comparable to those displayed by rats and mice and faster 
than several lizard and turtle species (Krekorian et al. 1968; Northcutt \& Heath 1973; Kirkish et al. 1979; Morris 1984; Schenk \& Morris 1985; Silva et al. 1992; Cremer et al. 1994; Holtzman et al. 1999; Day et al. 1999b, 2001; López et al. 2001; Punzo 2002; Paulissen 2008; LaDage et al. 2012; Noble et al. 2012).

The observed role of body size in influencing spatial learning ability was an unexpected outcome of this study. The role of size in relevance to past experience and anti-predator response offer plausible explanations, albeit ones that have not been reported elsewhere in the learning literature, and suggests that although our choice of reinforcer was ecologically relevant, it may have been a limitation in this study. As such, we suggest that future work considers the influence that variables like individual experience and behavioural type may have upon the reinforcer used to ensure that all subjects are equally and consistently motivated to perform (Rowe \& Healy 2014). Further investigation into the role of body size and condition is also warranted and it would be useful to conduct a spatial learning experiment utilising a range of size cohorts to better examine the relationship between size and learning performance.

\subsubsection{Conclusion and conservation implications}

We provide evidence that ambient temperature may affect learning performance in a cold-adapted reptile, the tuatara, though further work with a larger sample size is required to confirm this relationship. As tuatara have a possible lifespan in excess of 100 years, juveniles hatched in recent years have the potential to experience climate change projections for the $2100 \mathrm{~s}$, which include air temperature increases of up to $4.8^{\circ} \mathrm{C}$ (IPCC 2014). Our results suggest that although anthropogenic climate change may generate novel and potentially challenging environments, warmer temperatures could also improve the ability of tuatara to respond successfully via enhanced cognitive performance, although the positive effects of this may be countered by the effects of thermal stress at temperatures above those tested in this study (Williams et al. 2007; Amiel \& Shine 2012). We also suggest that an individual's size can influence performance in a spatial learning task via size-dependent differences in anti-predator response, or the impact that prior experience with larger conspecifics may have on response to a threatening stimulus. However, in the absence of behavioural assays and knowledge of 
prior juvenile interactions we cannot assert that behaviour/motivation affects learning performance. We suggest that future studies consider the roles that individual experience and behavioural type may play when utilising a predatory stimulus in a learning task. 


\section{Chapter Seven}

\section{Thesis summary and discussion: Using unprecedented conservation translocations to assess impacts of anthropogenic climate change on the tuatara (Sphenodon punctatus)}

\subsection{Introduction}

In the face of rising air temperatures and the associated abiotic and biotic effects predicted under anthropogenic climate change, it is vital that we predict the effects on particularly vulnerable species. Such knowledge can inform where future conservation efforts are best directed, what practices to avoid and, in terms of conservation translocations, which sites might be most suitable for reinforcements or the establishment of new populations. My research used a broad range of methodologies, from field observations and the collection of morphometric data to laboratory-based screening and genetic analysis of biological samples to experimental assessments under controlled conditions. These approaches allowed me to examine a number of perspectives where tuatara might be influenced by warmer climates, and how they might behave in a warmer world.

The tuatara is a species of particular interest as it is a evolutionarily-distinctive, range-restricted, cold-adapted ectotherm with a low reproductive output and is consequently limited in its possible responses to climate change. The presence of the tuatara lineage in New Zealand for the last $\sim 80$ million years indicates that this species, or at least its ancestors, persisted through past climate change events. However, current climate change is progressing at a rate unprecedented in the past 65 million years (Diffenbaugh \& Field 2013; IPCC 2014). Unaided dispersal to suitable climates is not feasible due to population isolation on offshore islands and within fenced sanctuaries. Given the rate of climate change and the infrequent reproduction and slow maturation of tuatara, genetic adaptation is also unlikely. This leaves in situ adjustment to the changing climate or assisted dispersal to more suitable locations (i.e. translocation) as the most viable options for species survival.

I studied the short-term success of tuatara translocations following movement to sanctuaries around the North Island of New Zealand that were both warmer (i.e. Cape 
Sanctuary, Young Nicks Head, Whangaokeno) and that experienced similar climates (i.e. Maungatautari) to the source site. I used observational studies to assess short-term translocation success as well as relationships between the act of translocation and local climates on enteric bacterial communities, parasites and tuatara behaviour. I also experimentally investigated how warmer temperatures might influence learning ability. Although the chapters within this thesis cover a broad range of fields, they are all linked by the unifying theme of possible impacts on tuatara from anthropogenic climate change. The results of this thesis are highly relevant to tuatara conservation and the goals set out in the Tuatara Recovery Plan (Gaze 2001). My work answers questions regarding the short-term outcomes of translocations of Stephens Island tuatara to warmer locations and how climate change may influence multiple aspects of tuatara biology.

\subsection{Chapter overviews}

\section{Chapter Two}

Survival and growth of Cook Strait tuatara (Sphenodon punctatus) following translocation to warmer locations: implications for the temporal effects of climate change

Post-translocation monitoring is fundamental to the assessment of translocation success and the rapid identification of potential threats to the translocated population. This chapter evaluated three short-term measures of translocation success to determine how tuatara populations translocated outside of their ecological region were progressing: 1) founder survival, 2) founder growth, and 3) reproduction. We gathered data on individual recaptures, morphometric measurements and evidence of reproduction over 2.5 years following release. While this is an insufficient timeframe over which to determine longterm translocation success it provides a useful interim measure of the populations' progress and suitability of the translocation sites.

We found moderate to high recapture numbers, growth of founders and evidence of reproduction at most sites, with greater mass increases observed at the warmer, less densely populated sites. Variable growth in the Cape Sanctuary adults suggested that comparatively high population density, intraspecific competition, and lower water 
availability could be responsible for substantial weight loss in multiple individuals and management recommendations were made to alleviate this issue. Overall we found that sites with warmer climates and lower population densities were potentially beneficial to translocated tuatara, likely through enhanced temperature-dependent and densitydependent growth rates. We conclude that tuatara may benefit from further translocations to warmer sites and from warming climates at currently cooler sites, though these populations will likely be more vulnerable to future air temperature increases and associated abiotic changes (e.g. increased incidence of drought). However, further monitoring of these populations is required to determine longer-term translocation success.

\section{Chapter Three}

The association between translocation (and associated climate shifts) and the prevalence of the gastrointestinal colonisers, Campylobacter spp. and Salmonella spp. in tuatara (Sphenodon punctatus)

A principal concern regarding anthropogenic climate change is that reptiles may become more susceptible to disease owing to changes in disease ecology dynamics, including the possibility that pathogens may show increased growth rates, survival, and transmission capabilities (Harvell et al. 2002). Equally, translocation-associated stressors and risk factors can lead to immunosuppression and make individuals more susceptible to infection (Jacobson 1993; Dickens et al. 2010; Kock et al. 2010). Salmonella spp. had not previously been detected in a live tuatara, possibly due to an innate resistance, and the presence of Campylobacter spp. was equivocal (Gartrell et al. 2007). Therefore, the translocations of tuatara from the cooler Stephens Island climate to multiple warmer sites offered the opportunity to test the effects of both translocation and climate warming on the prevalence of potentially pathogenic (Salmonella spp.) and commensal (Campylobacter spp.) bacteria.

We collected cloacal swabs from adult tuatara at each site on one to four occasions (depending on site access) over 2.5 years following release and tested them for both bacteria. We detected Salmonella Saintpaul on only one occasion in one individual and the soil at Cape Sanctuary, which indicated a very low prevalence of this bacteria and 
the potential for asymptomatic carriage in tuatara, but offered no insights into factors influencing Salmonella spp. prevalence. Campylobacter spp. prevalence was high across all sites, showing it to be a common commensal with a non-pathogenic association in tuatara and my results suggested no measurable impact of translocation or climate on Campylobacter spp. prevalence. That we were unable to confirm the genus/species being carried following gene sequencing suggests that tuatara might carry a novel Campylobacter species. As such, there is no evidence that translocation or warming climates will facilitate increased Salmonella spp. or Campylobacter spp. prevalence in tuatara, though we cannot extend this conclusion to other bacterial species, especially to changes in the full spectrum of intestinal or dermal microbiota.

\section{Chapter Four}

\section{Relationships between climate, population density and timing of translocation on parasites of tuatara (Sphenodon punctatus)}

The interplay between hosts and parasites are complex, with parasites found to negatively impact factors like growth, reproduction and survival, but also play important roles in population regulation and the maintenance of genetic diversity (Scott 1988; Hudson et al. 1992; Godfrey et al. 2008). Host-parasite interactions can be influenced by both climate and translocation-associated stressors, so translocations to warmer sites enable an assessment of tuatara parasite populations following translocation as well as how they might be impacted under anthropogenic climate change. We used visual counts and faecal floats to monitor the presence of tuatara ticks, trombiculid mites and intestinal parasites in tuatara following release at translocation sites.

Our results demonstrated significant short-term reductions in tick populations following translocation, which were attributed to the size of the release site and dispersal of tuatara away from the release location, with subsequent population recovery or decline attributed to a combination of thermal climate and population density affecting tick survival and transmission opportunities. An absence of mites in the translocated populations showed that removal of uninfected tuatara to sites lacking in local reservoirs effectively eradicated mites from the translocated populations. Few faecal samples were obtained, but the endoparasites identified were in agreement with previous reports from 
tuatara. The results for interactions between tuatara and the tuatara tick suggest that warmer climates may not have a substantial short-term effect on tick populations when host density is high, but associated abiotic factors may severely affect the survival of freeliving ticks when host density is low and reattachment opportunities are limited.

\section{Chapter Five}

Thermoregulatory behaviour and nocturnal activity in translocated populations of a cold-adapted reptile, the tuatara (Sphenodon punctatus), and conservation implications facing climate change

Behavioural modification is one way in which organisms may adjust to warming climates. Higher ambient temperatures may prove beneficial to tuatara (e.g. by enabling greater year round activity), but could also have negative consequences (e.g. increased drought or drier climates may restrict emergence from burrows). We used observational methods to document diurnal behaviour, and a spatially detailed monitoring technique (i.e. cotton spooling) to track the nocturnal activity of female tuatara at two of the translocation sites, as well as at the source site and a control site.

Our results showed that diurnal and nocturnal activity were both significantly influenced by air temperature, with nocturnal activity and diurnal emergence behaviour found to increase with rising temperature, up to a possible upper limit on emergence. Location preferences when outside of the burrow also varied with air temperature and habitat type and I found limited evidence that tuatara may have thermoregulated more effectively when the thermal climate was at the cooler and warmer extremes of the air temperatures recorded. Combined with climate data from the translocation sites, we found that rising temperatures under climate change may enable greater activity throughout the year, allowing more opportunities for fitness-associated behaviours, but may also impose behavioural constraints if air temperatures regularly exceed the suggested upper emergence threshold. 


\section{Chapter Six}

Will a warming climate produce "smarter" reptiles? Ambient temperature and learning performance in the tuatara (Sphenodon punctatus)

The capacity to quickly and flexibly adjust to novel challenges and changeable environments predicted under anthropogenic climate change could be highly advantageous and increase individual fitness and survival (Amiel \& Shine 2012). Learning ability is a crucial component of an animal's cognitive repertoire and underpins vital behaviours like predator avoidance and foraging. I investigated the impact of warm versus cool ambient test temperatures on the ability of juvenile tuatara to learn the location of an accessible retreat in a Y-maze as an assessment of how warmer climates might influence, and potentially benefit, cognitive ability in this species.

I found that test air temperature likely played a role in influencing learning ability, suggesting that warmer climate may improve the ability of tuatara to cope with challenging environments through enhanced cognitive performance. However, body size was a more influential factor, with smaller individuals found to be better learners, possibly due to size-dependent differences in anti-predator responses or the impact of prior experiences of hostile interactions with larger conspecifics. I suggest that further work is needed to replicate and build on these initial findings and that behavioural assays and the assessment of distinct size cohorts would prove useful in distinguishing between the effects of size and temperature.

\subsection{Discussion}

I used multiple conservation translocations of tuatara from Stephens Island to sanctuary sites outside of their ecological region to assess how warming climates predicted under anthropogenic climate change may impact aspects of tuatara physiology, behaviour, disease ecology and parasitology. I also used experimental assessments of ambient test temperature on learning ability to investigate how warmer temperatures might influence cognition in this cold-adapted reptile.

Most translocated populations were found to be progressing favourably in the short term and indicated that Stephens Island tuatara would be suitable for further 
translocations to mainland or island sanctuaries outside of their extant ecological region (Chapter Two). The encouraging progress of translocations to several sites with climates reflective of those predicted for much of New Zealand under climate change (i.e. up to $4^{\circ} \mathrm{C}$ warmer by the $2080^{\prime} \mathrm{s}$, combined with an increased incidence of drought; Reisinger et al. 2014) also suggests that rising air temperatures alone are not likely to directly affect survival of individual tuatara, though this does not rule out that extreme temperature events might. However, although evidence of reproduction was found at all translocation sites, the indirect effects of climate warming on tuatara reproductive success and sex ratios were not assessed as part of this study. Reproduction and the maintenance of an appropriate sex ratio is one component of a self-sustaining population and could be threatened by warming temperatures, thereby threatening population survival in warmer climates (Nelson et al. 2004b).

SVL growth was only substantial in the Whangaokeno population, but mass gain was observed in most translocated populations. Particularly high mass gains were observed in the Whangaokeno and Young Nicks Head Sanctuary tuatara, which were attributed to a potential interaction between enhanced temperature-dependent and density-dependent growth rates at these two sites (Chapter Two). We considered the variable changes in mass observed in the Cape Sanctuary population to be illustrative of high intraspecific competition for resources (e.g. invertebrate prey) producing individuals that gained weight ("winners") and individuals that lost substantial amounts of weight over time following release ("losers"). Similarly, a depletion of resources was considered partially responsible for a failed translocation of giant tortoises (Geochelone gigantea) within the Seychelles (Hambler 1994). That the contrast in mass change observed in the Cape Sanctuary population was not observed in the similarly dense Maungatautari Tautari Wetland population was thought to be due to the additional effects of climate. Maungatautari is a relatively humid site, whereas Cape Sanctuary was shown to be considerably drier, has no natural water source, and is prone to periods of drought over summer. The combined effects of a warm, dry climate and high population density could have depleted invertebrate populations within the tuatara enclosure at Cape Sanctuary, resulting in consequent competition for dwindling resources (Chapter Two). However, surveys of invertebrate populations are required to confirm this and experimental studies are required to explicitly test the effects of temperature, density and food supply on 
tuatara growth in a controlled environment. Management recommendations have since been proposed to alleviate this problem, but further monitoring is required to see if these recommendations have been implemented and to ascertain the current condition of adult tuatara at this site.

In uniting the various results from the different chapters of this thesis it is apparent that warmer climates within the scope of those documented here may substantially affect tuatara populations through increased temperature-dependent growth rates, potentially enhanced learning ability, as well as increased opportunities for year-round activity and the attainment of preferred body temperatures (Chapters Two, Five and Six). Equally, that we found no measurable effect of climate or translocation on prevalence of Salmonella spp. and Campylobacter spp. and no ill effects of colonisation with these bacteria suggests that they pose little risk to the populations studied under the various climatic conditions recorded (Chapter Three). However, it is apparent that the temperature-associated benefits described have also been mediated by other site-specific factors and illustrate that assessment of the tuatara's ability to persist through anthropogenic climate change will depend substantially on the individual sites at which they reside, especially in the absence of dispersal opportunities from fenced and island sites.

The Whangaokeno tuatara, the males in particular, showed substantially higher mass gains than observed at any other translocation site (Chapter Two). While thermal climate was suggested as a possible factor influencing the changes observed, population density was also considered to be important. It is also feasible that the considerably reduced tick (A. sphenodonti) populations documented on Whangaokeno could have contributed to the weight gains observed through reduced impacts on body condition and body mass as overall and larval tick loads have been found to be significantly associated with body condition in spring on Stephens Island (Godfrey et al. 2010a). The comparatively low tick numbers in the Whangaokeno population were attributed to a combination of climate, habitat structure and low host density affecting reduced off-host tick survival and reattachment opportunities (Chapter Four). This highlights that the complex and interactive effects of site-specific biotic and abiotic factors can influence multiple processes relevant to tuatara survival.

In contrast, Cape Sanctuary is similar to Whangaokeno in that it is comparatively warmer than Stephens Island and ZEALANDIA and its position on New Zealand's North 
East coast means it is also prone to drought. However, the higher population density at this site likely aided the recovery of tick populations, which in conjunction with intraspecific competition and depletion of resources (also partially due to population density) could have contributed to the declining weights observed in a large proportion of the population (Chapter Two). The high number of $>25^{\circ} \mathrm{C}$ degree days over summer at this site could also have restricted activity outside of the burrow and limited the amount of time that could be spent on fitness-related behaviours (i.e. foraging), which could also have contributed to the limited increases and substantial decreases in mass observed in this population when compared to similarly warm sites that experience lower numbers of $>25^{\circ} \mathrm{C}$ degree days (i.e. Whangaokeno and Young Nicks Head, Chapter Four). This demonstrates the importance of the thermal climate not only in terms of physiological but also behavioural impacts on tuatara, as well as alternative climatic and site-specific factors (e.g. humidity, density). It also emphasises that while warmer climates may have proven acceptable, there is an upper threshold, beyond which air temperatures will impose restrictions, and potential benefits are also dependent upon the presence/absence of other limiting factors.

\subsection{Limitations}

We faced multiple challenges associated with conducting research in the field at multiple sites on a cryptic species of high conservation concern. These challenges did impose some limits on the data collection in most aspects of the research detailed in this thesis. Firstly, access to the six study sites was governed by permit restrictions imposed by local and regional governing bodies (i.e. landowners, iwi and the Department of Conservation), which meant that some sites could not be visited in each survey season. Other limitations were associated with physical access to the sites. For instance, access to Stephens Island, a Nature Reserve of high conservation importance, is carefully moderated by the Department of Conservation to minimise disturbance to the local wildlife and habitat, as well as reduce the risk of pest incursion, so research visits were restricted to one per year at this site. Equally, transport to this site is by either boat or helicopter. While boat is the more cost-effective option, this mode of transport takes longer and is wholly dependent on suitable weather conditions in the Cook Strait and can therefore be delayed by several 
days both going to and leaving the island. Such delays are unavoidable but can jeopardise the viability of biological samples (i.e. cloacal swabs) collected on the island and prove problematic to field work scheduling when several sites must be visited in a short timeframe.

Incorporating visits of up to 10 days at three to six geographically distant study sites in one study season also proved logistically challenging at times. As this research formed my PhD thesis, site visits could not be distributed amongst separate research teams. Since I led each survey, this meant that sites were all surveyed at different times, though every effort was made to keep surveys as close together as possible within the same season. The processing of cloacal swabs had to begin within a short timeframe following collection (I set a maximum limit of five days based on advice from other researchers), which meant that I often had to return to the lab in Palmerston North for periods of up to a week during survey seasons to perform the required culturing and genetic analyses before returning to the field. However, in later survey seasons I avoided this issue by performing an initial round trip to collect cloacal swabs. I processed these in the lab, and then returned to sites to collect further samples and conduct behavioural work.

Technical problems regarding faulty dataloggers, unreliable climate data, and the drop-off of iButtons meant that some data were more limited. The technical faults demonstrate that backup environmental dataloggers or an alternative means of recording relative humidity would be highly advisable in future studies. The drop-off of iButtons was an unavoidable consequence of using a low risk method of attaching monitoring equipment to tuatara. There were also breakages of the cotton spools used to track nocturnal and diurnal activity that reduced the sample sizes for those analyses, but again, the use of a method that could fail was done in the interest of minimising risk to the tuatara and was considered an acceptable trade-off. Alternatives included GPS tracking units and radio telemetry, but these options were both substantially more expensive and would not have provided as much detail or precision as was provided by the cotton spools. Despite these limitations, I was able to obtain an integrated data set that allowed me to assess the potential impacts of translocation and warmer climates on multiple aspects of tuatara ecology. 


\subsection{Conservation implications and management recommendations}

The research conducted under the broad scope of this thesis can contribute to the conservation of tuatara, and potentially other cold-adapted reptiles, in numerous ways. Tuatara are range restricted and considered to be conservation dependent by the New Zealand Department of Conservation (Hitchmough et al. 2016). A substantial part of the Tuatara Recovery Plan and the conservation of this species relies on the translocation of individuals to restore populations at sites within the species' historical range, and it has been suggested that translocation may be necessary to mitigate extinction risk for species with temperature-dependent sex determination (Gaze 2001; Mitchell et al. 2008). The first contemporary tuatara translocation took place in 1995 and numerous translocations have taken place since to move tuatara to pest-free sites within the same ecological region (Towns et al. 2001; Nelson et al. 2002b, 2008; Gartrell et al. 2006; Miller et al. 2010). However, considering the threats posed by anthropogenic climate change, it is vital that we understand the potential consequences of exposure to warmer climates and the likelihood that populations of this cold-adapted species will survive. The October 2012 translocations offered the opportunity to assess how tuatara would cope following translocation to sites with climates representative of those predicted for New Zealand under climate change (Reisinger et al. 2014).

Similarly positive short-term progress following the use of conservation translocations has been noted in reptile species around the world, though long term data for many reptile translocations is lacking (Germano \& Bishop 2008; Ewen et al. 2014). While several studies and reviews suggest translocations can be used as a management tools to mitigate the negative impacts of climate change (Hoegh-Gulberg et al. 2008; Loss et al. 2010; Thomas 2011; Gallagher et al. 2014; Boyer et al. 2016), I found no studies in the literature that have used translocations as tools to study the effects that warming climates could have on a species. However, I found one example of a mitigation translocation of Agassiz's desert tortoises (Gopherus agassizii) within the United States to sites at elevations and with vegetation assemblages not usually associated with this species (Nussear et al. 2012). The authors suggest that translocation to alternative habitat may become increasingly necessary for this species as their natural habitat is lost to development, but may also offer insights in to how the species could adapt to climate 
change. However, post-translocation surveys found that animals released into atypical habitat dispersed to sites with vegetation more commonly associated with desert tortoises, so success in alternative habitats could not be assessed (Nussear et al. 2012). Nonethless, this indicates that future translocations could offer multiple benefits, both through the more immediate conservation of a species, and in enabling research into how best to conserve that species in the future.

In line with the short-term success of the majority of tuatara translocations studied, while translocations within Australia have been primarily focussed on birds and mammals (Mawson 2004), the Western Swamp tortoise (Pseudemydura umbrina) in Western Australia is one example of a (so far) successfully translocated reptile. This critically endangered tortoise shares several characteristics with the tuatara; it is longlived, slow to mature, and range restricted, thanks to a combination of predation by introduced mammals and habitat loss due to human development (Burbidge \& Kuchling 2004). Translocations to fenced sites began in the early 1990s and, although success cannot yet be properly evaluated due to the species' longevity (Short 2009), translocations have been held up as responsible for an increase in population size from 30 individuals in the mid 1980's to at least 110 in 2001 (Burbidge and Kuchling 2004). However, over 20 years of post-release monitoring will be required to determine long term survival and successful reproduction for this species (Dodd \& Seigel 1991; Short 2009).

As most translocated populations were found to be progressing favourably in the short term (and recommendations were made for Cape Sanctuary), there is no evidence to suggest that tuatara translocated from Stephens Island possess local adaptations that might prevent them from establishing at sites outside of their ecological region. As such, further translocations of animals from Stephens Island to similar translocation sites would be feasible and the results so far bode well for tuatara survival facing rising air temperatures within the scope of those predicted by the IPCC (IPCC 2014; Reisinger et al. 2014). However, there are valid concerns regarding their ability to cope with extreme temperature events and associated abiotic factors that are expected to increase in frequency (e.g. drought), and which have yet to be fully assessed (IPCC 2014; Reisinger et al. 2014). As the translocation of Stephens Island individuals to the cooler Orokonui Ecosanctuary site has been progressing well in the short term (Jarvie et al. 2015) and will 
likely allow for successful nest incubation in the future (Besson et al. 2012; Jarvie et al. 2014), further translocations to southern sites, which are not expected to experience the same high temperatures or abiotic extremes, could be a promising long-term conservation strategy worth further investigation.

The large population inhabiting Stephens Island makes it unlikely that the removal of adults for further translocations would be detrimental to the remaining individuals. Given that this population exhibited a density-dependent decline in body condition between 1949 and 2003 (Moore et al. 2007), the translocation of individuals could instead help relieve inter- and intraspecific competition in the areas from which animals are removed and aid the reversal of this decline. However, the declining condition observed in $56 \%$ of the adult tuatara released at Cape Sanctuary also illustrates the importance of providing tuatara with sufficiently large enclosures. Forty adult tuatara were translocated to Cape Sanctuary and released into a 2,000 $\mathrm{m}^{2}$ enclosure within the fenced 1.5 ha Cape Sanctuary Seabird Cell, with the objective that they would be allowed to disperse into the wider site after an initial acclimation period (akin to a soft release strategy). Dispersal into the 1.5 ha site would have resulted in a population density of 27 adult animals per hectare, rather than the $200 \mathrm{ha}^{-1}$ density that has been maintained within the smaller enclosure. In this warm, drought-prone climate, such a high population density has shown to be potentially detrimental and it is likely that, had tuatara been afforded the original space proposed, then results might have been more similar to those observed at other warm, but less densely populated, translocation sites (e.g. Whangaokeno and Young Nicks Head). We have yet to determine whether tuatara that have shown declining condition at Cape Sanctuary will recover, but it would be advisable to avoid high population densities at sites that are likely to experience stressful abiotic conditions (e.g. drought), which can impact prey populations and lead to the competitive exclusion of individuals. Accordingly, populations of invertebrates, the tuatara's primary prey, are influenced by humidity, with greater activity observed on warm and humid nights and populations negatively impacted following periods of drought (Walls 1981, 1983; New 2008).

Warmer environmental temperatures are thought to potentially increase the susceptibility of organisms to bacterial infection. However, we found no evident impact of translocation or climate on the gastrointestinal carriage of Salmonella spp. or Campylobacter spp., which bodes well for further translocations and predicted exposure 
of tuatara to warmer climates (Harvell et al. 2002; Reisinger et al. 2014). However, considering that we detected Salmonella Saintpaul carriage in a live tuatara, potential sources of Salmonella spp. should be considered at any prospective translocation sites. Exposure to potential pathogens in combination with environmental stressors could be a health concern for both tuatara populations and the people involved in the translocations (Teixeira et al. 2007).

The absence of trombiculid mites following translocation could be highly beneficial to tuatara considering their potential negative impact on tuatara body condition (Godfrey et al. 2010a). It has been proposed that removal of ectoparasites that pose a risk to tuatara survival might be a viable conservation strategy (Godfrey et al. 2010a). Therefore, if the elimination of mites is a desirable outcome, it might be as straightforward as translocating tuatara during the spring season when mites are not attached to individuals (Godfrey et al. 2008). However, there is debate around whether parasites should be conserved or removed during translocations. Some advocate that treatments (e.g. vaccinations, antiparasitics) should be used when translocating wildlife to prevent the release of vectors and pathogens into naïve local populations and reduce the susceptibility of translocated individuals to infection following release, particularly where parasitism or disease could impact conservation goals (Woodford 2000; Wobeser 2002; Kock et al. 2010; Almberg et al. 2012). Conversely, others suggest that parasites are fundamental components of biodiversity and ecosystem functioning, and that their removal or reduction could cause a cascade effect on other organisms in the biological community, including effects on competing parasite species, which could benefit from the removal of dominant competitors (Poulin 1999; Møller 2005; Hudson et al. 2006; Dobson et al. 2008; Thompson et al. 2010). Ultimately, there is no single solution, and management decisions will depend on whether a parasite is a threat to a host population or if their presence is an important feature of population and ecosystem health (Thompson et al. 2010).

We found no evidence to suggest a detrimental effect of mite absence on translocated tuatara populations and nothing to suggest that their absence enabled other ectoparasites to increase in prevalence or load. As such, elimination of trombiculid mites, which are capable of infesting multiple species and might pose a threat to naïve local populations, might be an acceptable course of action in tuatara translocations. However, 
a better understanding of their ecological role and community-level effects are required when advocating for or against mite elimination (Thompson et al. 2010). In contrast, elimination might not be a feasible option for the host-specific tuatara tick, an endangered parasite that is even more range-restricted than its host (Durden \& Keirans 1996; Miller et al. 2007; Buckley et al. 2012).

Short-term declines in tick populations might be unavoidable following translocation and could benefit tuatara via a brief reprieve from the negative impacts of parasitism. However, the majority of sites experienced subsequent recovery of tick populations to prevalences close to pre-translocation. As tick infestation can also reduce body condition and the substantial weight gains observed in the Whangaokeno population may have been partially due to low tick prevalence's and loads, it follows that tuatara populations might benefit from the removal of ticks prior to translocation. However, this action would have to be weighed against conservation goals for the equally (if not more) vulnerable tuatara tick. If tick conservation were an important component of a translocation then sites that would provide similar climates and host densities to the Whangaokeno site, at which tick population survival was impaired, would be unsuitable. The results from Chapter Four also illustrate the importance of repeat monitoring in gathering accurate information on parasite population change over time.

The consistently warmer temperatures of several translocation sites and positive relationships between air temperature, emergence and activity indicate that tuatara translocated to warmer sites may benefit from increased time spent at preferred body temperatures, greater emergence opportunities throughout the year and a reduction in the time and energy spent on thermoregulation, which could increase the time and energy available for other fitness-related activities (Huey 1974, 1982; Huey \& Slatkin 1976; Seebacher 1999). The removal of such constraints could ultimately improve survival and population viability at warmer sites. However, warmer climates like those at Cape Sanctuary are also likely to impose greater thermal constraints on tuatara during the summer season (indicated by the higher number of $>25^{\circ} \mathrm{C}$ degree days), which could lead to a reduction in overall activity and emergence compared to populations at cooler sites. Therefore I suggest that simple assessments of the thermal climate at proposed translocation sites might provide information on behavioural opportunities for tuatara, which might in turn influence translocation success. Equally, associated decreases in 
relative humidity might have a similarly restrictive effect and translocations to cooler or at least more humid climates may prove a more beneficial conservation strategy. Similarly, the availability of an artificial water source at warm, drought prone sites lacking in a natural water supply might help alleviate associated impacts on physiology and behaviour related to evaporative water loss. However, the use of artificial water sources could create additional risks, with increased transmission of parasites and disease in South Island saddlebacks (Philesturnus c. carunculatus) on New Zealand's Motuara Island thought to be due to congregation of individuals around limited numbers of water sources during dry periods (Hale \& Briskie 2009).

\subsection{Recommendations for future research}

The research presented in this thesis has addressed questions regarding aspects of tuatara disease ecology, behaviour, cognitive ability and parasitology under warmer climates and following translocation. While I was able to answer many of the questions posed, I was unable to investigate some aspects of climate and, equally, new questions have arisen. I suggest that the following work would extend the research presented in this thesis and advance the understanding of how anthropogenic climate change and translocation may impact tuatara populations.

\subsubsection{Long term progress of the October 2012 translocations}

As tuatara are a long-lived species with slow maturation and long reproductive cycles (breeding every four years on average on Stephens Island) it is well known that assessment of translocation success, the point at which a population becomes selfsustaining, will take much longer than the 2.5 years of monitoring that has contributed to this thesis. Continued monitoring is clearly needed and while annual monitoring is probably neither necessary nor feasible, I suggest that efforts should be made to intensively evaluate the progress of the translocations every five years at sites where the populations have been found to be progressing well, where effective pest control is in place, and where there is no apparent significant short-term risk from biotic or abiotic factors. Of course, if potential threats to the tuatara population become apparent, the 
frequency of monitoring should be increased as necessary. Such assessments should include the standard measures of progress used in Chapter One (i.e. morphometric measurements, recapture numbers, evidence of reproduction). As the translocations progress (e.g. in 10 years time), evidence of juvenile recruitment into the adult population should be sought. However, considering the continual declines in condition observed for over half of the population, I suggest that the adult population at Cape Sanctuary should be monitored annually. If the barriers surrounding the adult enclosure are removed or modified then subsequent monitoring of adult condition will also provide a measure of whether population density and intraspecific competition were negatively affecting individuals in the population, or if the decreasing weights could be attributed to alternative factors like climate or habitat.

\subsubsection{Influence of relative humidity on tuatara behaviour and ectoparasites}

Technical faults with our equipment meant we were unable to quantify the relationships between relative humidity and parasite survival following translocation (Chapter Four) or tuatara nocturnal and diurnal thermoregulatory behaviour (Chapter Five). Relative humidity is considered to have a substantial influence on the survival of free-living parasites (which are vulnerable to desiccation in dry environments; Oliver 1989) as well as the emergence and activity of tuatara (which are vulnerable to evaporative water loss; Hill 1982; Walls 1983; Corkery et al. 2014). Therefore, it would be informative to conservation management and future translocation decisions to understand the lower humidity thresholds to tuatara activity, especially as the incidence of drought is predicted to increase under anthropogenic climate change and reduced emergence could lead to reduced opportunities for fitness-related behaviours that could ultimately impair population viability (Reisinger et al. 2014; Corkery et al. 2014b). The possible reduction in tick numbers following translocation to Whangaokeno may have been due to a combination of tuatara population density and climate affecting reduced off-host survival and I have suggested that high temperature and low humidity at this East coast site may have been important factors. Therefore, whether conservation of $A$. sphenodonti or the loss of ectoparasites is a desirable outcome following translocation, knowledge of climatic factors that limit tick survival in wild populations would be useful. 
7.6.3 Effects of body size, ambient temperature and incubation temperature on tuatara learning ability

We found a significant effect of body size and a near significant effect of ambient test temperature on learning ability in juvenile tuatara. However, this work was based on a sample size of only 20 individuals and while small sample sizes can be unavoidable when working with a protected species like the tuatara, further work with a larger sample is required to build on this initial finding. Further investigation into the role of body size and condition is also warranted. Assessment of learning ability in different size cohorts under different temperature treatments would enable a better assessment of the relationship between size, ambient temperature and learning performance. Previous work has also found incubation temperatures to consistently influence learning ability in skinks (Amiel \& Shine 2012; Clark et al. 2013) and, given that climate change will likely lead to higher soil temperatures and thereby influence temperatures in the nest, an assessment of how incubation temperature might affect learning ability in the tuatara would provide a useful assessment of whether juveniles incubated at warmer sites may experience cognitive benefits. As males are produced from nests incubated at higher temperatures this might also provide insight into possible sex-dependent differences in cognitive ability, though an assessment of tuatara produced from clutches incubated within the $1^{\circ} \mathrm{C}$ transitional range (in which both sexes are produced) could help elucidate the role of sex versus incubation temperature. Evaluations of learning ability in wild adult populations located at climatically different sites would also offer a more ecologically valid assessment of how cognitive ability might be impacted by warmer climates.

7.6.4 Identification of Campylobacter spp. carried by tuatara and potential to investigate changes in dermal and enteric bacterial communities following translocation and/or climate change

The identification of Campylobacter spp. as common commensal bacteria in tuatara could be of use in monitoring changes in bacterial communities following translocation or climate change. Previous work has used Campylobacter spp. prevalence in multiple populations of the flightless New Zealand endemic bird, the takahe (Porphyrio hochstetteri), which are connected by ongoing translocations, to study variation, potential 
transmission routes and infection sources/sinks within the translocation network (Grange et al. 2014, 2015). As we were unable to identify the Campylobacter species associated with tuatara, potentially because tuatara are carrying an as yet unidentified species, we are currently unable to monitor patterns or changes in the Campylobacter spp. community in tuatara. However, if we are able to identify the species carried by tuatara, this knowledge could be used in a similar manner to that demonstrated by Grange et al. (2015) and we could investigate variation in Campylobacter spp. carriage with translocation, exposure opportunities, abiotic conditions or location-specific factors like proximity to farmland or water sources. Knowledge of infection patterns for commensal bacteria could also inform potential transmission routes for pathogenic bacteria, which could impact population survival. Similar but larger-scale investigations into the dermal and enteric microbiomes of tuatara in response to similar variables would likewise provide baseline knowledge of tuatara-associated microbes and insight into factors influencing these bacterial communities, which could aid the early detection of potential pathogens, identify sources of infection, and inform conservation management decisions (Waite et al. 2012).

\subsubsection{Translocations and supplementations using tuatara from northern populations}

The short-term success of the October 2012 translocations, which moved Stephens Island tuatara to sites outside of their extant ecological region, suggests that these animals did not possess local adaptations that prevented establishment and survival at (for the most part) warmer, drier sanctuary sites. As translocations of tuatara from extant northern populations might have been as logistically feasible, a next step would be to supplement existing populations or establish new populations using individuals sourced from northern sites. This would enable research into whether local adaptation to warmer climates might influence behavioural/physiological responses and survival following translocation from warmer northern ecological regions and would provide a useful comparison of how geographically distinct populations respond following movement to different thermal climates. Such studies could also provide insight into whether some populations are better suited for translocations to certain regions than others. 


\subsection{Conclusion}

The rate at which anthropogenic climate change is proceeding is a significant conservation concern, especially to cold-adapted, range-restricted species like the tuatara. I have used mainland translocations to sites outside of the Stephens Island tuatara population's extant ecological range to predict how the cold-adapted tuatara might be impacted by exposure to warmer, drier climate like those predicted for much of New Zealand under climate change. I have contributed to knowledge on tuatara disease ecology, parasitology, behaviour and cognitive ability and provided insight into the influence rising temperatures may have on conservation of tuatara, which is also relevant to other cold-adapted reptiles. I have also assessed the short-term success of translocations to climatically different sites and made recommendations for future conservation translocation management, which will be relevant to translocations of similarly range-restricted reptiles. I conclude that tuatara may experience initial physiological, behavioural and cognitive benefits under a warming climate, though associated abiotic factors may impose constraints on behaviour and pose substantial risks to persistence in warmer, droughtprone regions as climate change progresses. However, further monitoring is required to determine longer-term translocation success and continued survival of tuatara at these sites. These results will contribute to further research into the interactive effect of climatic and site-specific factors (e.g. humidity, habitat, population density) on tuatara persistence in a warming climate, as well as informing research on other cold-adapted, rangerestricted reptile species and the ongoing suitability of conservation translocations in mitigating climate change-associated risks. 


\section{References}

Ackman DM, Drabkin P, Birkhead G, Cieslak P. 1995. Reptile-associated salmonellosis in New York State. The Pediatric Infectious Disease Journal 14:955-958.

Addo-Bediako A, Chown SL, Gaston KJ. 2000. Thermal tolerance, climatic variability and latitude. Proceedings of the Royal Society of London B: Biological Sciences 267:739745.

Adler K, Phillips J. 1985. Orientation in a desert lizard (Uma notata): time-compensated compass movement and polarotaxis. Journal of Comparative Physiology A 156:547552.

Ahmad G, Zemenhof S. 1978. Serotonin as a growth factor for chick embryo brain. Life Sciences 22:963-970.

Alberts AC. 2007. Behavioral considerations of headstarting as a conservation strategy for endangered Caribbean rock iguanas. Applied Animal Behaviour Science 102:380391.

Alexander JE, Covich AP. 1991. Predation risk and avoidance behavior in two freshwater snails. The Biological Bulletin 180:387-393.

Alford RA, Richards SJ. 1999. Global amphibian declines: a problem in applied ecology. Annual Review of Ecology and Systematics 30:133-165.

Allan SA, Simmons LA, Burridge MJ. 1998. Establishment of the tortoise tick Amblyomma marmoreum (Acari: Ixodidae) on a reptile-breeding facility in Florida. Journal of Medical Entomology 35:621-624.

Allen MA, Fischer GJ. 1978. Ambient temperature effects on paired associate learning. Ergonomics 21:95-101.

Alley MR, Connolly JH, Fenwick SG, Mackereth GF, Leyland MJ, Rogers LE, Haycock M, Nicol C, Reed CEM. 2002. An epidemic of salmonellosis caused by Salmonella typhimurium DT160 in wild birds and humans in New Zealand. New Zealand Veterinary Journal 50:170-176.

Almberg ES, Cross PC, Dobson AP, Smith DW, Hudson PJ. 2012. Parasite invasion following host reintroduction: a case study of Yellowstone's wolves. Philosophical Transactions of the Royal Society B: Biological Sciences 367:2840-2851.

Altschul SF, Gish W, Miller W, Myers EW, Lipman DJ. 1990. Basic local alignment search 
tool. Journal of Molecular Biology 215:403-410.

Amiel JJ, Bao S, Shine R. 2016. The effects of incubation temperature on the development of the cortical forebrain in a lizard. Animal Cognition:1-9.

Amiel JJ, Lindstrom T, Shine R. 2014. Egg incubation effects generate positive correlations between size, speed and learning ability in young lizards. Animal Cognition 17:337347.

Amiel JJ, Shine R. 2012. Hotter nests produce smarter young lizards. Biology Letters 8:372374.

Andersen P, Moser El. 1995. Brain temperature and hippocampal function. Hippocampus 5:491-498.

Anderson A. 1996. Was Rattus exulans in New Zealand 2000 years ago? AMS Radiocarbon ages from Shag River Mouth. Archaeology in Oceania 31:178-184.

Anderson RM, May RM. 1979. Population biology of infectious diseases 1. Nature 280:361-367.

Andry ML, Luttges MW, Gamow RI. 1971. Temperature effects on spontaneous and evoked neural activity in the garter snake. Experimental Neurology 31:32-44.

Angilletta MJ. 2009. Thermal adaptation, a theoretical and empirical synthesis. Oxford University Press, New York, USA.

Angilletta MJ, Steury TD, Sears MW. 2004. Temperature, growth rate, and body size in ectotherms: Fitting pieces of a life-history puzzle. Integrative and Comparative Biology 44:498-509.

Applebaum SW, Heifetz Y. 1999. Density-dependent physiological phase in insects. Annual Review of Entomology 44:317-341.

ARASG [Australasian Reptile \& Amphibian Specialist Group]. 1996. Sphenodon punctatus. The IUCN Red List of Threatened Species 1996: e.T20613A9214781.

Ari T Ben, Neerinckx S, Gage KL, Kreppel K, Laudisoit A, Leirs H, Stenseth NC. 2011. Plague and climate: scales matter. PLoS Pathogens 7:e1002160.

Armstrong DP, McLean IG. 1995. New Zealand translocations: theory and practice. Pacific Conservation Biology 2:39-54.

Arneberg P, Skorping A, Grenfell B, Read AF. 1998. Host densities as determinants of abundance in parasite communities. Proceedings of the Royal Society of London B: Biological Sciences 265:1283-1289. 
Arnold EN. 1986. Mite pockets of lizards, a possible means of reducing damage by ectoparasites. Biological Journal of the Linnean Society 29:1-21.

Atkins KE, Travis JMJ. 2010. Local adaptation and the evolution of species' ranges under climate change. Journal of Theoretical Biology 266:449-457.

Aubret F, Shine R. 2009. Thermal plasticity in young snakes: how will climate change affect the thermoregulatory tactics of ectotherms? Journal of Experimental Biology 213:242-248.

Auffenberg W, Auffenberg T. 1990. The reptile tick Aponomma gervaisi (Acarina: Ixodidae) as a parasite of monitor lizards in Pakistan and India. Bulletin of the Florida Museum of Natural History, Biological Sciences 35:1-34.

Autumn K, De Nardo DF. 1995. Behavioral thermoregulation increases growth rate in a nocturnal lizard. Journal of Herpetology 29:157-162.

Avery RA, Bedford JD, Newcombe CP. 1982. The role of thermoregulation in lizard biology: predator efficiency in a temperate diurnal basker. Behavioural Ecology and Sociobiology 11:261-267.

Bager F, Petersen J. 1991. Sensitivity and specificity of different methods for the isolation of Salmonella from pigs. Acta Veterinaria Scandinavica 32:473-481.

Baggesen DL, Wegener HC, Christensen JP. 1996. Typing of Salmonella enterica serovar Saintpaul: an outbreak investigation. Apmis 104:411-418.

Baling M, Ji W, Gartrell B, Brunton DH. 2013a. Detection of Salmonella during the translocation of two endemic New Zealand lizard species within the Hauraki Gulf. New Zealand Journal of Zoology 40:249-254.

Baling M, van Winkel D, Habgood M, Ruffell J, Ji W, Ussher G. 2013b. A review of reptile research and conservation management on Tiritiri Matangi Island, New Zealand. New Zealand Journal of Ecology 37:272-281.

Baling M, Wedding C, Barry M, Ji W, Brunton DH. 2010. Re-introduction of shore skinks to offshore islands in the Auckland region, New Zealand. Pages 88-92 in P.S. Soorae, editor. Global Re-introduction Perspectives: Additional case-studies from around the globe. Gland, Switzerland: IUCN/SSC Reintroduction Specialist Group and Abu Dhabi, UAE: Environment Agency- Abu Dhabi.

Bally M, Garrabou J. 2007. Thermodependent bacterial pathogens and mass mortalities in temperate benthic communities: a new case of emerging disease linked to climate 
change. Global Change Biology 13:2078-2088.

Barrow PA, Jones MA, Thompson N. 2011. Salmonella. in C.L. Gyles, J.F. Prescott, J.G. Songer, and C.O. Thoen, editors. Pathogenesis of bacterial infections in animals. John Wiley \& Sons, Ames, Iowa, USA.

Bartholomew GA, Tucker VA. 1964. Size, body temperature, thermal conductance, oxygen consumption, and heart rate in Australian varanid lizards. Physiological Zoology 37:341-354.

Barwick RE. 1982. Observations on active thermoregulation in the tuatara, Sphenodon punctatus (Reptilia: Rhynchocephalia). Pages 225-236 in D.G. Newman, editor. New Zealand Herpetology. New Zealand Wildlife Service Occasional Publication No. 2, Wellington, New Zealand.

Basha PM, Sujitha NS. 2012. Combined impact of exercise and temperature in learning and memory performance of fluoride toxicated rats. Biological Trace Element Research 150:306-313.

Bates D, Maechler M, Bolker B, Walker S. 2016. Package "Ime4": Linear mixed-effects models using "Eigen" and S4. Available from https://cran.rproject.org/web/packages/Ime4/Ime4.pdf.

Bäumler AJ, Tsolis RM, Ficht TA, Adams LG. 1998. Evolution of host adaptation in Salmonella enterica. Infection and Immunity 66:4579-4587.

Bauwens L, Vercammen F, Bertrand S, Collard J, De Ceuster S. 2006. Isolation of Salmonella from environmental samples collected in the reptile department of Antwerp Zoo using different selective methods. Journal of Applied Microbiology 101:284-289.

Benavidez R, Neighbours S, Stenhouse V, Tichenor E. 2013. Assessment of reptiles and predator management in Cape Sanctuary, Hawkes Bay. Unpublished Masters report. Wellington, New Zealand.

Bennett AF. 1978. Activity metabolism of the lower vertebrates. Annual Review of Physiology 40:447-469.

Bennett AF. 1980. The thermal dependence of lizard behaviour. Animal Behaviour 28:752-762.

Benton MJ. 1987. The mite pockets of lizards. Nature 325:391-392.

Bertrand MR, Wilson ML. 1996. Microclimate-dependent survival of unfed adult Ixodes 
scapularis (Acari: Ixodidae) in nature: Life cycle and study design implications. Journal of Medical Entomology 33:619-627.

Besson AA, Cree A. 2010. A cold-adapted reptile becomes a more effective thermoregulator in a thermally challenging environment. Oecologica 163:571-581.

Besson AA, Cree A. 2011. Integrating physiology into conservation: an approach to help guide translocations of a rare reptile in a warming environment. Animal Conservation 14:28-37.

Besson AA, Nelson NJ, Nottingham CM, Cree A. 2012. Is cool egg temperature a limiting factor for the translocation of tuatara to southern New Zealand? New Zealand Journal of Ecology 36:90-99.

Bingman VP. 1992. The importance of comparative studies and ecological validity for understanding hippocampal structure and cognitive function. Hippocampus 2:213219.

Blair TA, Cree A, Skeaff CM. 2000. Plasma fatty acids, triacylglycerol and cholesterol of the tuatara (Sphenodon punctatus punctatus) from islands differing in the presence of rats and the abundance of seabirds. Journal of Zoology 252:463-472.

Blanchard B. 2002. Tuatara captive management plan and husbandry manual; Threatened Species Occcasional Publication 21. Department of Conservation, Wellington.

Blockley WV, Lyman J. 1950. Studies of human tolerance for extreme heat III: Mental performance under heat stress as indicated by addition and number checking tests. US Air Force, Air Materiel Command.

Bogert CM. 1949. Thermoregulation in reptiles, a factor in evolution. Evolution 3:195211.

Bogert CM. 1953. Body temperatures of the tuatara under natural conditions. Zoologica 38:63-64.

Bornemann R, Zerr DM, Heath J, Koehler J, Grandjean M, Pallipamu R, Duchin J. 2002. An outbreak of Salmonella serotype Saintpaul in a children's hospital. Infection Control 23:671-676.

Borsook D, Woolf CJ, Vellet AD. 1978. Temperature acclimation and learning in fish. Experientia 34:70-71.

Boyer S, Case BS, Lefort MC, Waterhouse BR, Wratten SD. 2016. Can ecosystem-scale translocations mitigate the impact of climate change on terrestrial biodiversity? 
Promises, pitfalls, and possibilities. F1000Research 5:146.

Boyles JG, Seebacher F, Smit B, McKechnie AE. 2011. Adaptive thermoregulation in endotherms may alter responses to climate change. Integrative and Comparative Biology:icr053.

Brattstrom BH. 1965. Body temperatures of reptiles. The American Midland Naturalist 73:376-422.

Brooks DR, Hoberg EP. 2007. How will global climate change affect parasite-host assemblages? Trends in Parasitology 23:571-574.

Brown CR, Brown MB, Rannala B. 1995. Ectoparasites reduce long-term survival of their avian host. Proceedings of the Royal Society of London B: Biological Sciences 262:313-319.

Brown D. 2000. Stephens Island: ark of the light. Cloudybay Publishing, Blenheim, New Zealand.

Broz P, Ohlson MB, Monack DM. 2012. Innate immune response to Salmonella typhimurium, a model enteric pathogen. Gut Microbes 3:62-70.

Buckley TR, Palma RL, Johns PM, Gleeson DM, Heath ACG, Hitchmough RA, Stringer IAN. 2012. The conservation status of small or less well known groups of New Zealand terrestrial invertebrates. New Zealand Entomologist 35:137-143.

Bull CM, Chilton NB, Sharrad RD. 1988. Risk of predation for two reptile tick species. Experimental and Applied Acarology 5:93-99.

Bull CM, Godfrey SS, Gordon DM. 2012. Social networks and the spread of Salmonella in a sleepy lizard population. Molecular Ecology 21:4386-4392.

Bull SA, Allen VM, Domingue G, Jørgensen F, Frost JA, Ure R, Whyte R, Tinker D, Corry JEL, Gillard-King J. 2006. Sources of Campylobacter spp. colonizing housed broiler flocks during rearing. Applied and Environmental Microbiology 72:645-652.

Burbidge AA and Kuchling G. 2004. Western swamp tortoise (Pseudemydura umbrina) recovery plan: $3^{\text {rd }}$ Edition. Wildlife Management Program No. 37, Department of Conservation and Land Management, Western Australia, Australia.

Burghardt GM. 1977. Learning processes in reptiles. Biology of the Reptilia 7:555-681.

Burghardt GM. 2013. Environmental enrichment and cognitive complexity in reptiles and amphibians: concepts, review, and implications for captive populations. Applied Animal Behaviour Science 147:286-298. 
Burke RL. 1992. Relocations, repatriations, and translocations of amphibians and reptiles: taking a broader view. Biological Conservation 62:350-357.

Burnham KP, Anderson DR. 2003. Model selection and multimodel inference: a practical information-theoretic approach. Springer-Verlag, New York, USA.

Burnham KP, Anderson DR. 2004. Multimodel inference understanding AIC and BIC in model selection. Sociological Methods \& Research 33:261-304.

Bush AO, Fernandez JC, Esch GW, Seed RJ. 2001. Parasitism. The diversity and ecology of animal parasites. Cambridge University Press, Cambridge, UK.

Calosi P, Bilton DT, Spicer JI. 2008. Thermal tolerance, acclimatory capacity and vulnerability to global climate change. Biology Letters 4:99-102.

Cambre RC, Green DE, Smith EE, Montali RJ, Bush M. 1980. Salmonellosis and arizonosis in the reptile collection at the National Zoological Park. Journal of the American Veterinary Medical Association 177:800-803.

Carazo P, Noble DWA, Chandrasoma D, Whiting MJ. 2014. Sex and boldness explain individual differences in spatial learning in a lizard. Proceedings of the Royal Society of London B: Biological Sciences 281:20133275.

Cardozo G, Chiaraviglio M. 2008. Landscape changes influence the reproductive behaviour of a key "capital breeder" snake (Boa constrictor occidentalis) in the Gran Chaco region, Argentina. Biological Conservation 141:3050-3058.

Carey C. 1993. Hypothesis concerning the causes of the disappearance of boreal toads from the mountains of Colorado. Conservation Biology 7:355-362.

Carmichael CK, Gillingham JC, Keall SN. 1989. Feeding ecology of the tuatara (Sphenodon punctatus) on Stephens Island based on niche diversification. New Zealand Journal of Zoology 16:269.

Castanet J, Newman DG, Saint Girons H. 1988. Skeletochronological data on the growth, age, and population structure of the tuatara, Sphenodon punctatus, on Stephens and Lady Alice Islands, New Zealand. Herpetologica 44:25-37.

Castinel A, Duignan PJ, Pomroy WE, Lopez-Villalobos N, Gibbs NJ, Chilvers BL, Wilkinson IS. 2007. Neonatal mortality in New Zealand sea lions (Phocarctos hookeri) at Sandy Bay, Enderby Island, Auckland Islands from 1998 to 2005. Journal of Wildlife Diseases 43:461-474.

Chambers DL, Hulse AC. 2006. Salmonella serovars in the herpetofauna of Indiana County, 
Pennsylvania. Applied and Environmental Microbiology 72:3771-3773.

Chao WL, Ding RJ, Chen RS. 1987. Survival of pathogenic bacteria in environmental microcosms. Chinese Journal of Microbiology and Immunology 20:339-348.

Charmantier A, McCleery RH, Cole LR, Perrins C, Kruuk LEB, Sheldon BC. 2008. Adaptive phenotypic plasticity in response to climate change in a wild bird population. Science 320:800-803.

Chen I, Hill JK, Ohlemüller R, Roy DB, Thomas CD. 2011. Rapid range shifts of species associated with high levels of climate warming. Science 333:1024-1026.

Chiles WD. 1958. Effects of elevated temperatures on performance of a complex mental task. Ergonomics 2:89-96.

Chilton NB, Bull CM. 1991. A comparison of the reproductive parameters of females of two reptile tick species. International Journal for Parasitology 21:907-911.

Chiodini RJ, Sundberg JP. 1981. Salmonellosis in reptiles: a review. American Journal of Epidemiology 113:494-499.

Choquenot D. 1991. Density-dependent growth, body condition, and demography in feral donkeys: testing the food hypothesis. Ecology 72:805-813.

Chown S, Slabber S, McGeoch M, Janion C, Leinaas HP. 2007. Phenotypic plasticity mediates climate change responses among invasive and indigenous arthropods. Proceedings of the Royal Society B: Biological Sciences 274:2531-2537.

Christian KA, Tracy CR. 1981. The effect of the thermal environment on the ability of hatchling Galapagos land iguanas to avoid predation during dispersal. Oecologia 49:218-223.

Cichoń M, Chadzińska M, Książek A, Konarzewski M. 2002. Delayed effects of cold stress on immune response in laboratory mice. Proceedings of the Royal Society of London B: Biological Sciences 269:1493-1497.

Clark BF, Amiel JJ, Shine R, Noble DWA, Whiting MJ. 2013. Colour discrimination and associative learning in hatchling lizards incubated at "hot" and "cold" temperatures. Behavioural Ecology and Sociobiology 68:239-247.

Clark NJ, Gordos MA, Franklin CE. 2008. Thermal plasticity of diving behavior, aquatic respiration, and locomotor performance in the Mary River turtle Elusor macrurus. Physiological and Biochemical Zoology 81:301-309.

Clark RG. 2001. Salmonella Brandenburg in cattle and humans. Pages 23-25 Proceedings 
of the 31 st Seminar of Sheep \& Cattle Veterinarians, New Zealand Veterinary Association, Christchurch.

Clark RG, Robinson RA, Alley MR, Nicol CM, Hathaway SC, Marchant RM. 2002. Salmonella in animals in New Zealand: the past to the future. New Zealand Veterinary Journal 50:57-60.

Clarke A, Pörtner HO. 2010. Temperature, metabolic power and the evolution of endothermy. Biological Reviews 85:703-727.

Clayton NS. 1990. Assortative mating in zebra finch subspecies, Taeniopygia guttata guttata and T. g. castanotis. Philosophical Transactions of the Royal Society B: Biological Sciences 330:351-370.

Clogg CC, Rubin DB, Schenker N, Schultz B, Weidman L. 1991. Multiple imputation of industry and occupation codes in census public-use samples using Bayesian logistic regression. Journal of the American Statistical Association 86:68-78.

Clusella-Trullas S, Terblanche JS, Chown SL. 2010. Phenotypic plasticity of locomotion performance in the seed harvester Messor capensis (Formicidae). Physiological and Biochemical Zoology 83:519-530.

Coburn B, Grassl GA, Finlay BB. 2007. Salmonella, the host and disease: a brief review. Immunology and Cell Biology 85:112-118.

Cooper WE, Wilson DS. 2007. Beyond optimal escape theory: Microhabitats as well as predation risk affect escape and refuge use by the phrynosomatid lizard Sceloporus virgatus. Behaviour 144:1235-1254.

Corkery I. 2012. Interspecific interactions: a case study using the tuatara-fairy prion association. Unpublished PhD thesis. Victoria University of Wellington, Wellington, New Zealand.

Corkery I, Bell BD, Nelson NJ. 2014a. Behavioral thermoregulation of the tuatara, Sphenodon punctatus, under hydric and digestive constraints. Herpetological Conservation and Biology 9:29-37.

Corkery I, Bell BD, Nelson NJ. 2014b. Investigating kleptothermy: A reptile-seabird association with thermal benefits. Physiological and Biochemical Zoology 87:216221.

Cowles RB, Bogert CM. 1944. A preliminary study of the thermal requirements of desert reptiles. Bulletin of the American Museum of Natural History 83:261-296. 
Cox RM, John-Alder HB. 2007. Increased mite parasitism as a cost of testosterone in male striped plateau lizards Sceloporus virgatus. Functional Ecology 21:327-334.

Cree A. 1994. Low annual reproductive output in female reptiles from New Zealand. New Zealand Journal of Zoology 21:351-372.

Cree A. 2014. Tuatara: biology and conservation of a venerable survivor. Canterbury University Press, Christchurch, New Zealand.

Cree A, Butler D. 1993. Tuatara recovery plan (Sphenodon spp.). Threatened Species Unit, Department of Conservation, Wellington, New Zealand.

Cree A, Thompson MB, Daugherty CH. 1995. Tuatara sex determination. Nature 375:543.

Cremer H, Lange R, Christoph A, Plomann M, Vopper G, Roes J, Brown R, Baldwin S, Kraemer P, Scheff S. 1994. Inactivation of the N-CAM gene in mice results in size reduction of the olfactory bulb and deficits in spatial learning. Nature 367:455-459.

Cristol DA, Switzer PV. 1999. Avian prey-dropping behavior. II. American crows and walnuts. Behavioral Ecology 10:220-226.

Crook IG. 1973. The tuatara, Sphenodon punctatus gray, on islands with and without populations of the Polynesian rat, Rattus exulans (Peale). Proceedings of the New Zealand Ecological Society 20:115-120.

Cullen DA, Sword GA, Dodgson T, Simpson SJ. 2010. Behavioural phase change in the Australian plague locust, Chortoicetes terminifera, is triggered by tactile stimulation of the antennae. Journal of Insect Physiology 56:937-942.

Cullen L, Bodmer RE, Pádua CV. 2000. Effects of hunting in habitat fragments of the Atlantic forests, Brazil. Biological Conservation 95:49-56.

Cumming GS, van Vuuren DP. 2006. Will climate change affect ectoparasite species ranges? Global Ecology and Biogeography 15:486-497.

Cunningham AA. 1996. Disease risks of wildlife translocations. Conservation Biology 10:349-353.

Curtis JL, Baird TA. 2008. Within-population variation in free-living adult and ectoparasitic larval Trombiculid mites on collared lizards. Herpetologica 64:189-199.

Daniel M, Kolár J, Zeman P, Pavelka K, Sádlo J. 1998. Predictive map of Ixodes ricinus highincidence habitats and a tick-borne encephalitis risk assessment using satellite data. Experimental and Applied Acarology 22:417-433.

Daszak P, Berger L, Cunningham AA, Hyatt AD, Green DE, Speare R. 1999. Emerging 
infectious diseases and amphibian population declines. Emerging Infectious Diseases $5: 735$.

Daszak P, Cunningham AA, Hyatt AD. 2000. Emerging infectious diseases of wildlife-threats to biodiversity and human health. Science 287:443-449.

Daugherty $\mathrm{CH}$, Cree A. 1990. Tuatara - A survivor from the dinosaur age. New Zealand Geographic 6: 66-86.

Davis KM, Burghardt GM. 2011. Turtles (Pseudemys nelsoni) learn about visual cues indicating food from experienced turtles. Journal of Comparative Psychology 125:404-410.

Dawbin WH. 1982. The tuatara Sphenodon punctatus : aspects of life history, growth and longevity. Pages $237-250$ in D.G. Newman, editor. New Zealand Herpetology. New Zealand Wildlife Service Occasional Publication No. 2, Wellington, New Zealand.

Day LB, Crews D, Wilczynski W. 1999a. Relative medial and dorsal cortex volume in relation to foraging ecology in congeneric lizards. Brain, Behavior and Evolution 54:314-322.

Day LB, Crews D, Wilczynski W. 1999b. Spatial and reversal learning in congeneric lizards with different foraging strategies. Animal Behaviour 57:393-407.

Day LB, Crews D, Wilczynski W. 2001. Effects of medial and dorsal cortex lesions on spatial memory in lizards. Behavioural Brain Research 118:27-42.

Day LB, Ismail N, Wilczynski W. 2003. Use of position and feature cues in discrimination learning by the whiptail lizard (Cnemidophorus inornatus). Journal of Comparative Psychology 117:440-448.

De Hamel FA, Mclnnes HM. 1971. Lizards as vectors of human salmonellosis. Journal of Hygiene 69:247-253.

De Kloet ER, Joëls M, Holsboer F. 2005. Stress and the brain: from adaptation to disease. Nature Reviews Neuroscience 6:463-475.

de la Fuente J, Estrada-Pena A, Venzal JM, Kocan KM, Sonenshine DE. 2008. Overview: ticks as vectors of pathogens that cause disease in humans and animals. Frontiers in Bioscience 13:6938-6946.

de la Peña E, Mälkiä A, Vara H, Caires R, Ballesta JJ, Belmonte C, Viana F. 2012. The influence of cold temperature on cellular excitability of hippocampal networks. PLoS ONE 7:e52475. 
DeCoursey TE, Cherny VV. 1998. Temperature dependence of voltage-gated $\mathrm{H}+$ currents in human neutrophils, rat alveolar epithelial cells, and mammalian phagocytes. The Journal of General Physiology 112:503-522.

Deutsch CA, Tewksbury JJ, Huey RB, Sheldon KS, Ghalambor CK, Haak DC, Martin PR. 2008. Impacts of climate warming on terrestrial ectotherms across latitude. Proceedings of the National Academy of Sciences 105:6668-6672.

DeWitt CB. 1967. Precision of thermoregulation and its relation to environmental factors in the desert iguana, Dipsosaurus dorsalis. Physiological Zoology 40:49-66.

DeWitt TJ, Scheiner SM. 2004. Phenotypic plasticity: functional and conceptual approaches. Oxford University Press, New York, USA.

Dickens MJ, Delehanty DJ, Romero LM. 2010. Stress: an inevitable component of animal translocation. Biological Conservation 143:1329-1341.

Diffenbaugh NS, Field CB. 2013. Changes in ecologically critical terrestrial climate conditions. Science 341:486-492.

Dobson A, Foufopoulos J. 2001. Emerging infectious pathogens of wildlife. Philosophical Transactions of the Royal Society B: Biological Sciences 356:1001-1012.

Dobson A, Lafferty KD, Kuris AM, Hechinger RF, Jetz W. 2008. Homage to Linnaeus: how many parasites? How many hosts? Proceedings of the National Academy of Sciences 105:11482-11489.

DOC. 2006. Island sanctuaries in the Marlborough Sounds. Available from http://www.doc.govt.nz/documents/about-doc/concessions-andpermits/conservation-revealed/island-sanctuaries-marlborough-sounds-lowres.pdf (accessed November 30, 2015).

Dodd Jr. KD, Seigel RA. 1991. Relocation, repatriation, and translocation of amphibians and reptiles: Are they conservation strategies that work? Herpetologica 47:336-350.

Duffield GA, Bull CM. 1996. Microhabitat choice and its role in determining the distribution of the reptile tick Amblyomma vikirri. Australian Journal of Ecology 21:255-263.

Dumbleton LJ. 1943. A new tick from the tuatara (Sphenodon punctatus). New Zealand Journal of Science and Technology 24:185b-190b.

Durden LA, Keirans JE. 1996. Host-parasite coextinction and the plight of tick conservation. American Entomologist 42:87-91. 
East $\mathrm{KT}$, East MR, Daugherty CH. 1995. Ecological restoration and habitat relationships of reptiles on Stephens Island, New Zealand. New Zealand Journal of Zoology 22:249261.

Easterling DR, Horton B, Jones PD, Peterson TC, Karl TR, Parker DE, Salinger MJ, Razuvayev V, Plummer N, Jamason P, Folland CK. 1997. Maximum and minimum temperature trends for the globe. Science 277:364-367.

Eberhart-Phillips J, Walker N, Garrett N, Bell D, Sinclair D, Rainger W, Bates M. 1997. Campylobacteriosis in New Zealand: results of a case-control study. Journal of Epidemiology and Community Health 51:686-691.

Esch GW, Gibbons JW, Bourque JE. 1975. An analysis of the relationship between stress and parasitism. American Midland Naturalist 93:339-353.

Evans SE, Prasad GVR, Manhas BK. 2001. Rhynchocephalians (Diapsida: Lepidosauria) from the Jurassic Kota Formation of India. Zoological Journal of the Linnean Society 133:309-334.

Evans SE. 2003. At the feet of the dinosaurs: the early history and radiation of lizards. Biological Reviews 78: 513-551.

Ewen JG, Soorae PS, Canessa S. 2014. Reintroduction objectives, decisions and outcomes: global perspectives from the herpetofauna. Animal Conservation 17:74-81.

Ewen JG, Thorogood R, Nicol C, Armstrong DP, Alley M. 2007. Salmonella typhimurium in hihi, New Zealand. Emerging Infectious Diseases 13:788-790.

Fayer R, Trout JM, Jenkins MC. 1998. Infectivity of Cryptosporidium parvum oocysts stored in water at environmental temperatures. The Journal of Parasitology 84:1165-1169.

Fischer J, Lindenmayer DB. 2000. An assessment of the published results of animal relocations. Biological Conservation 96:1-11.

Fitzgerald LA, Treglia ML, Angeli N, Hibbitts TJ, Leavitt DJ, Subalusky AL, Lundgren I, HillisStarr Z. 2015. Determinants of successful establishment and post-translocation dispersal of a new population of the critically endangered St. Croix ground lizard (Ameiva polops). Restoration Ecology 23:776-786.

Ford NB, Seigel RA. 1989. Phenotypic plasticity in reproductive traits: evidence from a viviparous snake. Ecology 70:1768-1774.

Franco A, Hendriksen RS, Lorenzetti S, Onorati R, Gentile G, Dell'Omo G, Aarestrup FM, Battisti A. 2011. Characterization of Salmonella occurring at high prevalence in a 
population of the land iguana Conolophus subcristatus in Galapagos Islands, Ecuador. PLOS ONE 6:e23147.

Franklin L, Fielding JE, Gregory J, Gullan L, Lightfoot D, Poznanski SY, Vally H. 2009. An outbreak of Salmonella typhimurium 9 at a school camp linked to contamination of rainwater tanks. Epidemiology and Infection 137:434-440.

Frederich M, Pörtner HO. 2000. Oxygen limitation of thermal tolerance defined by cardiac and ventilatory performance in spider crab, Maja squinado. American Journal of Physiology-Regulatory, Integrative and Comparative Physiology 279:R1531-R1538.

Freeberg TM. 1999. Spatial associations provide a context for social learning of courtship patterns in brown-headed cowbirds (Molothrus ater). Journal of Comparative Psychology 113:327-332.

Fremouw T, Jackson-Smith P, Kesner RP. 1997. Impaired place learning and unimpaired cue learning in hippocampal-lesioned pigeons. Behavioral Neuroscience 111: 963975.

French JW. 1942. The effect of temperature on the retention of a maze habit in fish. Journal of Experimental Psychology 31:79-87.

French NP, Midwinter A, Holland B, Collins-Emerson J, Pattison R, Colles F, Carter P. 2009. Molecular epidemiology of Campylobacter jejuni isolates from wild-bird fecal material in children's playgrounds. Applied and Environmental Microbiology 75:779783.

Friedman CR, Torigian C, Shillam PJ, Hoffman RE, Heltze D, Beebe JL, Malcolm G, DeWitt WE, Hutwagner L, Griffin PM. 1998. An outbreak of salmonellosis among children attending a reptile exhibit at a zoo. The Journal of Pediatrics 132:802-807.

Gaalema DE. 2011. Visual discrimination and reversal learning in rough-necked monitor lizards (Varanus rudicollis). Journal of Comparative Psychology 125:246-249.

Gallagher RV, Hancock N, Makinson RO, Hogbin T. 2014. Assisted colonisation as a climate change adaptation tool. Report to the Biodiversity Hub of the New South Wales Office of Environment \& Heritage, Australia.

Gans C. 1983. Is Sphenodon punctatus a maladapted relict? Pages 613-620 in A. Rhodin and K. Miyata, editors. Advanced in Herpetology and Evolutionary Biology: Essays in Honour of Ernest E. Williams. Museum of Comparative Biology, Cambridge, Massachusetts, USA. 
Gans C, Gillingham JC, Clark DL. 1984. Courtship, mating and male combat in tuatara, Sphenodon punctatus. Journal of Herpetology 18:194-197.

Garrick LD, Lang JW, Herzog HA. 1978. Social signals of adult American alligators. Bulletin of the American Museum of Natural History 160:155-192.

Garris Gl. 1984. Colonization and life cycle of Amblyomma variegatum (Acari: Ixodidae) in the laboratory in Puerto Rico. Journal of Medical Entomology 21:86-90.

Gartrell BD, Jillings E, Adlington BA, Mack H, Nelson NJ. 2006. Health screening for a translocation of captive-reared tuatara (Sphenodon punctatus) to an island refuge. New Zealand Veterinary Journal 54:344-349.

Gartrell BD, Youl JM, King CM, Bolotovski I, McDonald WL, Nelson NJ. 2007. Failure to detect Salmonella species in a population of wild tuatara (Sphenodon punctatus). New Zealand Veterinary Journal 55:134-136.

Gaulin SJC. 1992. Evolution of sex difference in spatial ability. American Journal of Physical Anthropology 35:125-151.

Gaze P. 2001. Tuatara recovery plan, 2001-2011. Threateneed Species Recovery Plan 47. Biodiversity Recovery Unit, Deptartment of Conservation, Wellington, New Zealand.

Gaze P, Cash B. 2008. A history of wildlife translocations in the Marlborough Sounds. Department of Conservation Occasional publication no. 72. Nelson, New Zealand.

Geinisman Y. 2000. Structural synaptic modifications associated with hippocampal LTP and behavioral learning. Cerebral Cortex 10:952-962.

Germano JM, Bishop PJ. 2008. Suitability of amphibians and reptiles for translocation. Conservation Biology 23:7-15.

Germano JM, Field KJ, Griffiths RA, Clulow S, Foster J, Harding G, Swaisgood RR. 2015. Mitigation-driven translocations: are we moving wildlife in the right direction? Frontiers in Ecology and the Environment 13:100-105.

Giacomelli M, Piccirillo A. 2014. Pet reptiles as potential reservoir of Campylobacter species with zoonotic potential. The Veterinary Record 174.

Gibbons JW, Scott DE, Ryan TJ, Buhlmann KA, Tuberville TD, Metts BS, Greene JL, Mills T, Leiden Y, Poppy S. 2000. The global decline of reptiles, déjà vu amphibians. BioScience 50:653-666.

Gibbs GW. 2006. Ghosts of Gondwana: the history of life in New Zealand. Craig Potton Publishing, Nelson, New Zealand. 
Gibson CWD, Hamilton J. 1984. Population processes in a large herbivorous reptile: the giant tortoise of Aldabra atoll. Oecologia 61:230-240.

Gibson J, Hall E, Johnson E, McLean C. 2015. Assessment of Cape Sanctuary juvenile tuatara population: Health, habitat, and management. Unpublished Masters report. Victoria University of Wellington, Wellington, New Zealand.

Gilbert MJ, Kik M, Timmerman AJ, Severs TT, Kusters JG, Duim B, Wagenaar JA. 2014. Occurrence, diversity, and host association of intestinal Campylobacter, Arcobacter, and Helicobacter in reptiles. PLoS ONE 9:e101599.

Gillingham JC, Carmichael C, Miller T. 1995. Social behavior of the tuatara, Sphenodon punctatus. Herpetological Monographs 9:5-16.

Gillingham JC, Miller TJ. 1991. Reproductive ethology of the tuatara: Sphenodon punctatus: applications in captive breeding. International Zoo Yearbook 30:157-164.

Gliwicz ZM, Umana G. 1994. Cladoceran body size and vulnerability to copepod predation. Limnology and Oceanography 39:419-424.

Godfrey SS, Bull CM, Nelson NJ. 2008. Seasonal and spatial dynamics of ectoparasite infestation of a threatened reptile, the tuatara (Sphenodon punctatus). Medical and Veterinary Entomology 22:374-385.

Godfrey SS, Moore JA, Nelson NJ, Bull CM. 2010a. Unravelling causality from correlations: revealing the impacts of endemic ectoparasites on a protected species (tuatara). Parasitology 137:275-286.

Godfrey SS, Moore JA, Nelson NJ, Bull CM. 2010b. Social network structure and parasite infection patterns in a territorial reptile, the tuatara (Sphenodon punctatus). International Journal for Parasitology 40:1575-1585.

Godfrey SS, Nelson NJ, Bull CM. 2011a. Ecology and dynamics of the blood parasite, Hepatozoon tuatarae (Apicomplexa), in tuatara (Sphenodon punctatus) on Stephens Island, New Zealand. Journal of Wildlife Diseases 47:126-139.

Godfrey SS, Nelson NJ, Bull CM. 2011b. Microhabitat choice and host-seeking behaviour of the tuatara tick, Amblyomma sphenodonti (Acari: Ixodidae). New Zealand Journal of Ecology 35:52-60.

Goff ML, Loomis RB, Ainsworth R. 1987. Redescription of Neotrombicula naultini (Dumbleton, 1947) and descriptions of two new species of chiggers from New Zealand (Acari: Trombiculidae). New Zealand Journal of Zoology 14:385-390. 
Gómez A, Nichols E. 2013. Neglected wildlife: Parasitic biodiversity as a conservation target. International Journal for Parasitology: Parasites and Wildlife 2:222-227.

Grange ZL, Gartrell BD, Biggs PJ, Nelson NJ, Marshall JC, Howe L, Balm MGM, French NP. 2015. Using a common commensal bacterium in endangered Takahe as a model to explore pathogen dynamics in isolated wildlife populations. Conservation Biology 29:1327-1336.

Grange ZL, Van Andel M, French NP, Gartrell BD. 2014. Network analysis of translocated takahe populations to identify disease surveillance targets. Conservation Biology 28:518-528.

Grant JWA, Imre I. 2005. Patterns of density-dependent growth in juvenile streamdwelling salmonids. Journal of Fish Biology 67:100-110.

Gray JE. 1842. Descriptions of two hitherto unrecorded species of reptiles from New Zealand; presented to the British Museum by Dr. Dieffenbach. Zoological Miscellany 4:57-72.

Gray JS, Dautel H, Estrada-Peña A, Kahl O, Lindgren E. 2009. Effects of climate change on ticks and tick-borne diseases in Europe. Interdisciplinary Perspectives on Infectious Diseases 2009:1-12.

Gray TZ. 2011. Update: reptiles and Salmonella. Journal of Exotic Pet Medicine 20:14-17.

Grether WF. 1973. Human performance at elevated environmental temperatures. Aerospace Medicine 44:747-755.

Grieco F, van Noordwijk AJ, Visser ME. 2002. Evidence for the effect of learning on timing of reproduction in blue tits. Science 296:136-138.

Griffin AS, Evans CS. 2003. Social learning of antipredator behaviour in a marsupial. Animal Behaviour 66:485-492.

Griffith B, Scott JM, Carpenter JW, Reed C. 1989. Translocation as a species conservation tool: Status and strategy. Science 245:477-480.

Gruber MAM. 2007. Conservation of tuatara (Sphenodon): an evaluation of the survival and growth of artificially incubated, head-started juveniles. Unpublished BSc (Hons) thesis. Victoria University of Wellington, Wellington, New Zealand.

Günther A. 1867. Contribution to the anatomy of Hatteria (Rhynchocephalus, Owen). Philosophical Transactions of the Royal Society of London 157:595-629.

Hale KA, Briskie JV. 2009. Rapid recovery of an island population of the threatened South 
Island Saddleback Philesturnus c. carunculatus after a pathogen outbreak. Bird Conservation International 19:239-253.

Halekoh U, Højsgaard S. (2014). A kenward-roger approximation and parametric bootstrap methods for tests in linear mixed models - the R package pbkrtest. Journal of Statistical Software 59: 1-30.

Hambler C. 1994. Giant tortoise Geochelone gigantea translocation to Curieuse Island (Seychelles): Success or failure? Biological Conservation 69:293-299.

Hancock PA. 1981. Heat stress impairment of mental performance: a revision of tolerance limits. Aviation, Space, and Environmental Medicine 52:177-180.

Hancock PA. 1982. Task categorization and the limits of human performance in extreme heat. Aviation, Space, and Environmental Medicine 53:778-784.

Hancock PA. 1989. A dynamic model of stress and sustained attention. Human Factors: The Journal of the Human Factors and Ergonomics Society 31:519-537.

Hancock PA, Vasmatzidis I. 2003. Effects of heat stress on cognitive performance: the current state of knowledge. International Journal of Hyperthermia 19:355-372.

Haraga A, Ohlson MB, Miller SI. 2008. Salmonellae interplay with host cells. Nature Reviews Microbiology 6:53-66.

Hare KM, Cree A. 2010. Exploring the consequences of climate-induced changes in cloud cover on offspring of a cool-temperate viviparous lizard. Biological Journal of the Linnean Society 101:844-851.

Harvell CD, Mitchell CE, Ward JR, Altizer S, Dobson AP, Ostfeld RS, Samuel MD. 2002. Climate warming and disease risks for terrestrial and marine biota. Science 296:2158-2162.

Harvey S, Greenwood JR. 1985. Isolation of Campylobacter fetus from a pet turtle. Journal of Clinical Microbiology 21:260-261.

Haskell A, Graham TE, Griffin CR, Hestbeck JB. 1996. Size related survival of headstarted redbelly turtles (Pseudemys rubriventris) in Massachusetts. Journal of Herpetology 30:524-527.

Hay JM, Sarre SD, Lambert DM, Allendorf FW, Daugherty CH. 2010. Genetic diversity and taxonomy: a reassessment of species designation in tuatara (Sphenodon: Reptilia). Conservation Genetics 11:1063-1081.

Heath ACG. 2006. A reptile tick, Aponomma sphenodonti Dumbleton (Acari: Ixodidae), 
parasitic on the tuatara, Sphenodon punctatus Gray (Reptilia: Rhyncocephalia), in New Zealand: observations on its life history and biology. Systematic and Applied Acarology 11:3-12.

Heath ACG. 2016. Biology, ecology and distribution of the tick, Haemaphysalis longicornis Neumann (Acari: Ixodidae) in New Zealand. New Zealand Veterinary Journal 64:1020.

Heatwole H. 1982. Panting and other responses to high temperature in the tuatara, Sphenodon punctatus. Pages 251-269 in D.G. Newman, editor. New Zealand Herpetology. New Zealand Wildlife Service Occasional Publication No. 2, Wellington, New Zealand.

Hennessy K, Fitzharris B, Bates BC, Harvey N, Howden SM, Hughes L, Salinger J, Warrick R. 2007. Australia and New Zealand. Pages 507-540 in M.L. Parry, O.F. Canziani, J.P. Palutikof, P.J. van der Linden, and C.E. Hanson, editors. Climate change 2007: Impacts, adaptation and vulnerability. Contribution of Working Group II to the Fourth Assessment Report of the Intergovernmental Panel on Climate Change. Cambridge University Press, Cambridge, UK.

Herbert JDK, Godfrey SS, Bull CM, Menz RI. 2010. Developmental stages and molecular phylogeny of Hepatozoon tuatarae, a parasite infecting the New Zealand tuatara, Sphenodon punctatus and the tick, Amblyomma sphenodonti. International Journal for Parasitology 40:1311-1315.

Hetherington TE. 1989. Use of vibratory cues for detection of insect prey by the sandswimming lizard Scincus scincus. Animal Behaviour 37:290-297.

Hill L. 1982. Water relations and excretion of the tuatara, Sphenodon punctatus: an overview. In D.G. Newman, editor. New Zealand Herpetology: proceedings of a symposium held at Victoria University of Wellington, January 1980. New Zealand Wildlife Service Occasional Publication No. 2, New Zealand.

Hitchmough R, Barr B, Monks J, Lettink M, Reardon J, Tocher M, van Winkel D, Rolfe J. 2016. Conservation status of New Zealand reptiles, 2015. New Zealand Threat Classification Series. Wellington, New Zealand.

Hoare JM, Pledger S, Keall SN, Nelson NJ, Mitchell NJ, Daugherty CH. 2006. Conservation implications of a long-term decline in body condition of the Brothers Island tuatara (Sphenodon guntheri). Animal Conservation 9:456-462. 
Hochachka PW, Somero GN. 2002. Biochemical adaptation: mechanism and process in physiological evolution. Oxford University Press, New York, USA.

Hoegh-Guldberg O, Hughes L, McIntyre S, Lindenmayer DB, Parmesan C, Possingham HP, Thomas CD. Assisted colonization and rapid climate change. Science 321:345-346.

Hoff GL, White FH. 1977. Salmonella in reptiles: isolation from free-ranging lizards (Reptilia, Lacertilia) in Florida. Journal of Herpetology 11:123-129.

Hoffman AA, Chown SL, Clusella-Trullas S. 2013. Upper thermal limits in terrestrial ectotherms: how constrained are they? Functional Ecology 27:934-949.

Hoffman AA, Sgro CM. 2011. Climate change and evolutionary adaptation. Nature 470:479-485.

Holdaway RN, Worthy TH. 1997. A reappraisal of the late Quaternary fossil vertebrates of Pyramid Valley Swamp, North Canterbury, New Zealand. New Zealand Journal of Zoology 24:69-121.

Holding ML, Frazier JA, Taylor EN, Strand CR. 2012. Experimentally altered navigational demands induce changes in the cortical forebrain of free-ranging Northern Pacific Rattlesnakes (Crotalus o. oreganus). Brain, Behavior and Evolution 79:144-154.

Holtzman DA, Harris TW, Aranguren G, Bostock E. 1999. Spatial learning of an escape task by young corn snakes, Elaphe guttata guttata. Animal Behaviour 57:51-60.

Hudson P, Greenman J. 1998. Competition mediated by parasites: biological and theoretical progress. Trends in Ecology \& Evolution 13:387.

Hudson PJ, Dobson AP, Lafferty KD. 2006. Is a healthy ecosystem one that is rich in parasites? Trends in Ecology \& Evolution 21:381-385.

Hudson PJ, Newborn D, Dobson AP. 1992. Regulation and stability of a free-living hostparasite system: Trichostrongylus tenuis in red grouse. I. Monitoring and parasite reduction experiments. Journal of Animal Ecology 61:477-486.

Huey RB. 1974. Behavioral thermoregulation in lizards: importance of associated costs. Science 184:1001-1003.

Huey RB. 1982. Temperature, physiology, and the ecology of reptiles. Physiology C: Physiological Ecology 12:25-91.

Huey RB. 1991. Physiological consequences of habitat selection. American Naturalist 137:S91-S115.

Huey RB, Janzen FJ. 2008. Climate warming and environmental sex determination in 
tuatara: the last of the Sphenodontians? Proceedings of the Royal Society B: Biological Sciences 275:2181-2183.

Huey RB, Kingsolver JG. 1989. Evolution of thermal sensitivity of ectotherm performance.

Trends in Ecology \& Evolution 4:131-135.

Huey RB, Pianka ER. 1977. Seasonal variation in thermoregulatory behavior and body temperature of diurnal Kalahari lizards. Ecology 58:1066-1075.

Huey RB, Slatkin M. 1976. Cost and benefits of lizard thermoregulation. Quarterly Review of Biology 51:363-384.

Hughes P. 1967. The thermal relations of two nocturnal reptiles: the tuatara, Sphenodon punctatus and the leaf-tailed gecko, Phyllurus platurus. Unpublished B.Sc. Honours, University of Sydney, Sydney.

Hughes TP, Baird AH, Bellwood DR, Card M, Connolly SR, Folke C, Grosberg R, HoeghGuldberg O, Jackson JBC, Kleypas J. 2003. Climate change, human impacts, and the resilience of coral reefs. Science 301:929-933.

Hurlbert AH, Liang Z. 2012. Spatiotemporal variation in avian migration phenology: citizen science reveals effects of climate change. PLoS ONE 7:e31662.

Ikram R, Chambers S, Mitchell P, Brieseman MA, Ikam OH. 1994. A case control study to determine risk factors for Campylobacter infection in Christchurch in the summer of 1992-3. The New Zealand Medical Journal 107:430-432.

Inglis GD, Kalischuk LD. 2003. Use of PCR for direct detection of Campylobacter species in bovine feces. Applied and Environmental Microbiology 69:3435-3447.

Inglis GD, McAllister TA, Larney FJ, Topp E. 2010. Prolonged survival of Campylobacter species in bovine manure compost. Applied and Environmental Microbiology 76:1110-1119.

[IPCC] Intergovernmental Panel on Climate Change. 2014. Summary for Policymakers. Page 1-31 in CoreWritingTeam, R.K. Pachauri, and L.A. Meyer, editors. Climate Change 2014: Synthesis Report. Contribution of Working Groups I, II and III to the Fifth Assessment Report of the Intergovernmental Panel on Climate Change. IPCC, Geneva, Switzerland.

[IUCN/SSC] International Union for Conservation of Nature/Species Survival Commission. 1998. IUCN guidelines for re-introductions. (P.S. Soorae, editor). IUCN, Gland, Switzerland \& Cambridge, UK. 
[IUCN/SSC] International Union for Conservation of Nature/Species Survival Commission.

2013. Guidelines for reintroductions and other conservation translocations. Version 1.0. (P.S. Soorae, editor). IUCN Species Survival Commission, Gland, Switzerland.

Iveson JB, Shellam GR, Bradshaw SD, Smith DW, Mackenzie JS, Mofflin RG. 2009. Salmonella infections in Antarctic fauna and island populations of wildlife exposed to human activities in coastal areas of Australia. Epidemiology and Infection 137:858-870.

Jacobson ER. 1993. Implications of infectious diseases for captive propagation and introduction programs of threatened/endangered reptiles. Journal of Zoo and Wildlife Medicine 24:245-255.

Jäderlund L, Sessitsch A, Arthurson V. 2010. Persistence of two Campylobacter jejuni strains in soil and on spinach plants. Applied and Environmental Soil Science 2011.

Jakob-Hoff R. 1998. Kiwi parasite survey. Kokako 5:12.

Jankowski JE, Robinson SK, Levey DJ. 2010. Squeezed at the top: interspecific aggression may constrain elevational ranges in tropical birds. Ecology 91:1877-1884.

Janssens LA, Stoks R. 2014. Non-pathogenic aquatic bacteria activate the immune system and increase predation risk in damselfly larvae. Freshwater Biology 59:417-426.

Jarvie S, Besson AA, Seddon PJ, Cree A. 2014. Assessing thermal suitability of translocation release sites for egg-laying reptiles with temperature-dependent sex determination: a case study with tuatara. Animal Conservation 17:48-55.

Jarvie S, Senior AM, Adolph SC, Seddon PJ, Cree A. 2015. Captive rearing affects growth but not survival in translocated juvenile tuatara. Journal of Zoology 297: 184-193. Johnson-Delaney CA, Mader DR. 2006. Reptile zoonoses and threats to public health. Pages 20-33 in D.R. Mader, editor. Reptile Medicine and Surgery, 2nd edition. WB Saunders, Missouri, USA.

Johnston IA, Bennett AF. 1996. Animals and temperature: Phenotypic and evolutionary adaptation. Cambridge University Press, New York, USA.

Johnston IA. 1993. Phenotypic plasticity of fish muscle to temperature change. Pages 322340 in J.C. Rankin and F.B. Jensen, editors. Fish Ecophysiology. Springer Science \& Business Media, Dordrecht, Netherlands.

Johnston IA, Temple GK. 2002. Thermal plasticity of skeletal muscle phenotype in ectothermic vertebrates and its significance for locomotory behaviour. Journal of 
Experimental Biology 205:2305-2322.

Jones J, Helliwell P, Beekman M, Maleszka R, Oldroyd B. 2005. The effects of rearing temperature on developmental stability and learning and memory in the honey bee, Apis mellifera. Journal of Comparative Physiology A 191:1121-1129.

Jones ME, Tennyson AJ, Worthy JP, Evans SE, Worthy TH. 2009. A Sphenodontine (Rhynchocephalia) from the Miocene of New Zealand and palaeobiogeography of the tuatara (Sphenodon). Proceedings of the Royal Society of London B: Biological Sciences 276:1385-1390.

Kahneman D. 1973. Attention and effort. Prentice-Hall, Englewood Cliffs, New Jersey, USA.

Kakela R, Mattila M, Hermansson M, Haimi P, Uphoff A, Paajanen V, Somerharju P, Vornanen M. 2008. Seasonal acclimatization of brain lipidome in a eurythermal fish (Carassius carassius) is mainly determined by temperature. American Journal of Physiology - Regulatory, Integrative and Comparative Physiology 294:R1716-R1728. Karl TR, Knight RW, Gallo KP, Peterson TC, Jones PD, Kukla G, Plummer N, Razuvayev V, Lindseay J, Charlson RJ. 1993. A new perspective on recent global warming: asymmetric trends of daily maximum and minimum temperature. Bulletin of the American Meteorological Society 74:1007-1023.

Karl TR, Kukla G, Razuvayev VN, Changery MJ, Quayle RG, Heim RR, Easterling DR, Fu CB. 1991. Global warming: Evidence for asymmetric diurnal temperature change. Geophysical Research Letters 18:2253-2256.

Karvonen A, Rintamäki P, Jokela J, Valtonen ET. 2010. Increasing water temperature and disease risks in aquatic systems: climate change increases the risk of some, but not all, diseases. International Journal for Parasitology 40:1483-1488.

Kasuya T. 1991. Density dependent growth in North Pacific sperm whales. Marine Mammal Science 7:230-257.

Kearney M, Predavec M. 2000. Do nocturnal ectotherms thermoregulate? A study of the temperate gecko Christinus marmoratus. Ecology 81:2984-2996.

Kearney M, Shine R, Porter WP. 2009. The potential for behavioral thermoregulation to buffer "cold-blooded" animals against climate warming. Proceedings of the National Academy of Sciences 106:3835-3840.

Kearse M, Moir R, Wilson A, Stones-Havas S, Cheung M, Sturrock S, Buxton S, Cooper A, 
Markowitz S, Duran C, Thierer T, Ashton B, Meintjes P, Drummond A. 2012. Geneious Basic: an integrated and extendable desktop software platform for the organization and analysis of sequence data. Bioinformatics 28:1647-1649.

Kerr GD, Bull CM. 2006. Interactions between climate, host refuge use, and tick population dynamics. Parasitology research 99:214-222.

Kikillus KH, Gartrell BD, Motion E. 2011. Prevalence of Salmonella spp., and serovars isolated from captive exotic reptiles in New Zealand. New Zealand Veterinary Journal 59:174-178.

King BJ, Monis PT. 2007. Critical processes affecting Cryptosporidium oocyst survival in the environment. Parasitology 134:309-323.

King CM. 1990. The Handbook of New Zealand Mammals. Oxford University Press, Auckland, New Zealand.

Kirkish PM, Fobes JL, Richardson AM. 1979. Spatial reversal learning in the lizard Coleonyx variegatus. Bulletin of the Psychonomic Society 13:265-267.

Kis A, Huber L, Wilkinson A. 2015. Social learning by imitation in a reptile (Pogona vitticeps). Animal Cognition 18:325-331.

Klompen H, Dobson SJ, Barker SC. 2002. A new subfamily, Bothriocrotoninae n. subfam., for the genus Bothriocroton Keirans, King \& Sharrad, 1994 status amend.(Ixodida: Ixodidae), and the synonymy of Aponomma Neumann, 1899 with Amblyomma Koch, 1844. Systematic Parasitology 53:101-107.

Klukowski M, Nelson CE. 2001. Ectoparasite loads in free-ranging northern fence lizards, Sceloporus undulatus hyacinthinus: effects of testosterone and sex. Behavioral Ecology and Sociobiology 49:289-295.

Kock RA, Woodford MH, Rossiter PB. 2010. Disease risks associated with the translocation of wildlife. Revue Scientifique et Technique de l'OIE 29:329-350.

Koplan JP, Deen RD, Swanston WH, Tota B. 1978. Contaminated roof-collected rainwater as a possible cause of an outbreak of salmonellosis. Journal of Hygiene 81:303-309. Krekorian CO, Vance VJ, Richardson AM. 1968. Temperature-dependent maze learning in the desert iguana, Dipsosaurus dorsalis. Animal Behaviour 16:429-436.

Kutz SJ, Hoberg EP, Polley L, Jenkins EJ. 2005. Global warming is changing the dynamics of Arctic host-parasite systems. Proceedings of the Royal Society of London B: Biological Sciences 272:2571-2576. 
Labruna MB, Amaku M, Metzner JA, Pinter A, Ferreira F. 2003. Larval behavioral diapause regulates life cycle of Amblyomma cajennense (Acari: Ixodidae) in Southeast Brazil. Journal of Medical Entomology 40:170-178.

Labruna MB, Pinter A, Teixeira RHF. 2004. Life cycle of Amblyomma cooperi (Acari: Ixodidae) using capybaras (Hydrochaeris hydrochaeris) as hosts. Experimental and Applied Acarology 32:79-88.

Labruna MB, Souza SLP, Menezes AC, Horta MC, Pinter A, Gennari SM. 2002. Life-cycle and host specificity of Amblyomma tigrinum (Acari: Ixodidae) under laboratory conditions. Experimental and Applied Acarology 26:115-125.

LaDage LD, Riggs BJ, Sinervo B, Pravosudov VV. 2009. Dorsal cortex volume in male sideblotched lizards, Uta stansburiana, is associated with different space use strategies. Animal Behaviour 78:91-96.

LaDage LD, Roth TC, Cerjanic AM, Sinervo B, Pravosudov VV. 2012. Spatial memory: are lizards really deficient? Biology Letters 8:939-941.

Lafferty KD. 2009. The ecology of climate change and infectious diseases. Ecology 90:888900.

Laland KN, Williams K. 1997. Shoaling generates social learning of foraging information in guppies. Animal Behaviour 53:1161-1169.

Lawson PA, Secoy DM. 1991. The use of solar cues as migratory orientation guides by the plains garter snake, Thamnophis radix. Canadian Journal of Zoology 69:2700-2702.

Leal M, Powell BJ. 2012. Behavioural flexibility and problem-solving in a tropical lizard. Biology Letters 8:28-30.

Leighton FA. 2002. Health risk assessment of the translocation of wild animals. Revue Scientifique et Technique de I'OIE 21:187-195.

Lemons DE, Crawshaw LI. 1985. Behavioral and metabolic adjustments to low temperatures in the largemouth bass (Micropterus salmoides). Physiological Zoology 58:175-180.

Lenhardt ML. 1981. Evidence for auditory localization ability in the turtle. Journal of Auditory Research 21:255-261

Levin ML. 2015. Amblyomma spp. Available from http://www.merckvetmanual.com /mvm/integumentary_system/ticks/Amblyomma_spp.html (accessed November 15, 2015). 
Lin JW, Lo HY, Wang HC, Shaner PJL. 2014. The effects of mite parasitism on the reproduction and survival of the Taiwan field mice (Apodemus semotus). Zoological Studies 53:79.

Lin M, Zhao D, Hrabovsky A, Pedrosa E, Zheng D, Lachman HM. 2014. Heat shock alters the expression of schizophrenia and autism candidate genes in an induced pluripotent stem cell model of the human telencephalon. PloS ONE 9:e94968.

Lindgren E, Jaenson TGT. 2006. Lyme borreliosis in Europe: influences of climate and climate change, epidemiology, ecology and adaptation measures. WHO Regional Office for Europe, Copenhagen, Denmark.

Lindsay SW, Birley MH. 1996. Climate change and malaria transmission. Annals of Tropical Medicine and Parasitology 90:573-588.

Linton D, Owen RJ, Stanley J. 1996. Rapid identification by PCR of the genus Campylobacter and of five Campylobacter species enteropathogenic for man and animals. Research in Microbiology 147: 707-718.

Logan CA, Buckley BA. 2015. Transcriptomic responses to environmental temperature in eurythermal and stenothermal fishes. Journal of Experimental Biology 218:19151924.

Lohmann K, Lohmann C. 1996. Orientation and open-sea navigation in sea turtles. Journal of Experimental Biology 199:73-81.

Lohoefener R, Lohmeier L. 1986. Experiments with gopher tortoise (Gopherus polyphemus) relocation in southern Mississippi. Herpetological Review 17:37-40.

López J, Gómez Y, Rodríguez F, Broglio C, Vargas J, Salas C. 2001. Spatial learning in turtles. Animal Cognition 4:49-59.

López JC, Rodríguez F, Gómez Y, Vargas JP, Broglio C, Salas C. 2000. Place and cue learning in turtles. Animal Learning \& Behavior 28:360-372.

López JC, Vargas JP, Gómez Y, Salas C. 2003. Spatial and non-spatial learning in turtles: the role of medial cortex. Behavioural Brain Research 143:109-120.

Losos JB. 1987. Postures of the military dragon (Ctenophorus isolepis) in relation to substrate temperature. Amphibia-Reptilia 8:419-423.

Loss SR, Terwilliger LA, Peterson AC. 2010. Assisted colonization: Integrating conservation strategies in the face of climate change. Biological Conservation 144:92-100.

Lowell BB, Spiegelman BM. 2000. Towards a molecular understanding of adaptive 
thermogenesis. Nature 404:652-660.

Lucon-Xiccato T, Bisazza A. 2014. Discrimination reversal learning reveals greater female behavioural flexibility in guppies. Biology Letters 10:20140206.

MacAvoy ES, McGibbon LM, Sainsbury JP, Lawrence H, Wilson CA, Daugherty CH, Chambers GK. 2007. Genetic variation in island populations of tuatara (Sphenodon spp) inferred from microsatellite markers. Conservation Genetics 8:305-318.

MacBrayne R. 2002. Tuatara at home on island. New Zealand Herald. Available from http://www.nzherald.co.nz/nz/news/article.cfm?c_id=1\&objectid=940319.

Mackintosh MA, Briskie JV. 2005. High levels of hatching failure in an insular population of the South Island robin: a consequence of food limitation? Biological Conservation 122:409-416.

Mackworth NH. 1961. Researches on the measurement of human performance. Pages 174-331 in H.W. Sinaiko, editor. Selected papers on human factors in the design and use of control systems. Dover Publications, Inc., New York, USA.

MacLeod CJ, Paterson AM, Tompkins DM, Duncan RP. 2010. Parasites lost-do invaders miss the boat or drown on arrival? Ecology Letters 13:516-527.

MacNab V, Barber I. 2012. Some (worms) like it hot: fish parasites grow faster in warmer water, and alter host thermal preferences. Global Change Biology 18:1540-1548.

Macphail EM. 1982. Brain and intelligence in vertebrates. Clarendon Press, Oxford, UK.

Maguire EA, Woollett K, Spiers HJ. 2006. London taxi drivers and bus drivers: A structural MRI and neuropsychological analysis. Hippocampus 16:1091-1101.

Mahida YR, Rose F, Chan WC. 1997. Antimicrobial peptides in the gastrointestinal tract. Gut 40:161-163.

Man SM. 2011. The clinical importance of emerging Campylobacter species. Nature Reviews Gastroenterology and Hepatology 8:669-685.

Manrod J, Hartdegen R, Burghardt G. 2008. Rapid solving of a problem apparatus by juvenile black-throated monitor lizards (Varanus albigularis albigularis). Animal Cognition 11:267-273.

Marcellini DL, Jenssen TA. 1991. Avoidance learning by the curly-tailed lizard, Leiocephalus schreibersi: Implications for anti-predator behavior. Journal of Herpetology 25:238241.

Marin C, Ingresa-Capaccioni S, González-Bodi S, Marco-Jiménez F, Vega S. 2013. Free- 
living turtles are a reservoir for Salmonella but not for Campylobacter. PLoS ONE 8:e72350.

Markwell TJ. 1998. Relationship between tuatara Sphenodon punctatus and fairy prion Pachyptila turtur densities in different habitats on Takapourewa (Stephens Island), Cook Strait, New Zealand. Marine Ornithology 26:81-83.

Marler P. 1997. Three models of song learning: evidence from behavior. Journal of Neurobiology 33:501-516.

Martinez J, Merino S. 2011. Host-parasite interactions under extreme climatic conditions. Current Zoology 57:390-405.

Maschinski J, Duquesnel J. 2007. Successful reintroductions of the endangered long-lived Sargents cherry palm, Pseudophoenix sargentii, in the Florida Keys. Biological Conservation 134:122-129.

Massot M, Clobert J, Pilorge T, Lecomte J, Barbault R. 1992. Density dependence in the common lizard: demographic consequences of a density manipulation. Ecology 73:1742-1756.

Mawdsley JR, O'Malley R, Ojima DS. 2009. A review of climate-change adaptation strategies for wildlife management and biodiversity conservation. Conservation Biology 23:1080-1089.

Mawson PR. 2004. Translocations and fauna reconstruction sites: Western Shield review - February 2003. Conservation Science Western Australia 5: 108-121.

Maynard ME, Chung C, Comer A, Nelson K, Tran J, Werries N, Barton EA, Spinetta M, Leasure JL. 2016. Ambient temperature influences the neural benefits of exercise. Behavioural Brain Research 299:27-31.

Mazerolle MJ. 2016. Package "AICcmodavg": Model selection and multimodel inference

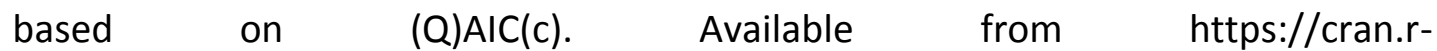
project.org/web/packages/AICcmodavg/AICcmodavg.pdf.

McAllister AK. 2000. Cellular and molecular mechanisms of dendrite growth. Cerebral Cortex 10:963-973.

McCoy ED, Osman N, Hauch B, Emerick A, Mushinsky HR. 2014. Increasing the chance of successful translocation of a threatened lizard. Animal Conservation 17:56-64.

Mclnnes HM. 1971. Salmonella Saintpaul infection of sheep with lizards as possible reservoirs. New Zealand Veterinary Journal 19:163-164. 
McKenna PB. 2003. An annotated checklist of ecto-and endoparasites of New Zealand reptiles. Surveillance 30:18-25.

McKenzie KL. 2007. Returning tuatara (Sphenodon punctatus) to the New Zealand mainland. Unpublished Masters thesis. Victoria University of Wellington, Wellington, New Zealand.

McLennan J. 2013. Cape Sanctuary: Te Matau a Maui. Internal Cape Sanctuary Report 2008-2013.

McLennan JA, Dew L, Miles J, Gillingham N, Waiwai R. 2004. Size matters: predation risk and juvenile growth in North Island brown kiwi (Apteryx mantelli). New Zealand Journal of Ecology 28:241-250.

McNab BK, Auffenberg W. 1976. The effect of large body size on the temperature regulation of the Komodo dragon, Varanus komodoensis. Comparative Biochemistry and Physiology Part A: Physiology 55:345-350.

McWilliams SR, Karasov WH. 2001. Phenotypic flexibility in digestive system structure and function in migratory birds and its ecological significance. Comparative Biochemistry and Physiology Part A: Molecular \& Integrative Physiology 128:577-591.

MEIT. 2013. Sanctuary Mountain Maungatautari. Available from http://www. sanctuarymountain.co.nz (accessed November 30, 2015).

Mello RSR, Besson AA, Hare KM, Fay V, Smith E, Cree A. 2013. Adjustment of juvenile tuatara to a cooler, southern climate: operative temperatures, emergence behaviour and growth rate. New Zealand Journal of Zoology 40:290-303.

Memmott J, Martinez ND, Cohen JE. 2000. Predators, parasitoids and pathogens: species richness, trophic generality and body sizes in a natural food web. Journal of Animal Ecology 69:1-15.

Menegaz RA, Sublett SV, Figueroa SD, Hoffman TJ, Ravosa MJ. 2009. Phenotypic plasticity and function of the hard palate in growing rabbits. The Anatomical Record 292:277284.

Mermin J, Hoar B, Angulo FJ. 1997. Iguanas and Salmonella marina infection in children: a reflection of the increasing incidence of reptile-associated salmonellosis in the United States. Pediatrics 99:399-402.

Mermin J, Hutwagner L, Vugia D, Shallow S, Daily P, Bender J, Koehler J, Marcus R, Angulo FJ. 2004. Reptiles, amphibians, and human Salmonella infection: a population-based, 
case-control study. Clinical Infectious Diseases 38:S253-S261.

Middleton DMRL, La Flamme AC, Gartrell BD, Nelson NJ. 2014. Reptile reservoirs and seasonal variation in the environmental presence of Salmonella in an island ecosystem, Stephens Island, New Zealand. Journal of Wildlife Diseases 50:655-659. Middleton DMRL, Minot EO, Gartrell BD. 2010. Salmonella enterica serovars in lizards of New Zealands offshore islands. New Zealand Journal of Ecology 34:247-252.

Middleton DMRL, Nelson NJ, Gartrell BD, La Flamme AC. 2015. Presence of antibodies to Salmonella in tuatara (Sphenodon punctatus) sera. Comparative Immunology, Microbiology and Infectious Diseases 41:17-27.

Miller HC, Conrad AM, Barker SC, Daugherty CH. 2007. Distribution and phylogenetic analyses of an endangered tick, Amblyomma sphenodonti. New Zealand Journal of Zoology 34:97-105.

Miller KA, Bell TP, Germano JM. 2014. Understanding publication bias in reintroduction biology by assessing translocations of New Zealand's herpetofauna. Conservation Biology 28:1045-1056.

Miller KA, Gruber MAM, Keall SN, Blanchard B, Nelson NJ. 2010. Changing taxonomy and the need for supplementation in the management of re-introductions of Brothers Island tuatara in Cook Strait, New Zealand. In P.S. Soorae, editor. Global reintroduction perspectives: additional case-studies from around the globe. Gland, Switzerland: IUCN/SSC Reintroduction Specialist Group and Abu Dhabi, UAE: Environment Agency- Abu Dhabi.

Miller KA, Miller HC, Moore JA, Mitchell NJ, Cree A, Allendorf FW, Sarre SD, Keall SN, Nelson NJ. 2012. Securing the demographic and genetic future of tuatara through assisted colonization. Conservation Biology 26:790-798.

Miller KA, Nelson NJ, Smith HG, Moore JA. 2009. How do reproductive skew and founder group size affect genetic diversity in reintroduced populations? Molecular Ecology 18:3792-3802.

Mitani JC, Rodman PS. 1979. Territoriality: the relation of ranging pattern and home range size to defendability, with an analysis of territoriality among primate species. Behavioral Ecology and Sociobiology 5:241-251.

Mitchell MA, Shane SM. 2001. Salmonella in reptiles. Seminars in Avian and Exotic Pet Medicine 10:25. 
Mitchell NJ, Kearney MR, Nelson NJ, Porter WP. 2008. Predicting the fate of a living fossil: how will global warming affect sex determination and hatching phenology in tuatara? Proceedings of the Royal Society of London B: Biological Sciences 275:21852193.

Mitchell NJ, Nelson NJ, Cree A, Pledger S, Keall SN, Daugherty CH. 2006. Support for a rare pattern of temperature-dependent sex determination in archaic reptiles: evidence from two species of tuatara (Sphenodon). Frontiers in Zoology 3: 9.

Moir ML, Vesk PA, Brennan KEC, Poulin R, Hughes L, Keith DA, McCarthy MA, Coates DJ. 2012. Considering extinction of dependent species during translocation, ex situ conservation, and assisted migration of threatened hosts. Conservation Biology 26:199-207.

Møller AP. 2005. Parasitism and the regulation of host populations. Pages $43-53$ in F. Thomas, F. Renaud, and J.F. Guégan, editors. Parasitism and ecosystems. Oxford University Press, Oxford, UK.

Møller AP. 2010. Host-parasite interactions and vectors in the barn swallow in relation to climate change. Global Change Biology 16:1158-1170.

Moller AP, Nielsen JT. 2007. Malaria and risk of predation: A comparative study of birds. Ecology 88:871-881.

Moore JA. 2008. Fitness implications of the mating system and reproductive ecology of tuatara. Unpublished PhD thesis, Victoria University of Wellington, Wellington, New Zealand.

Moore JA, Daugherty CH, Godfrey SS, Nelson NJ. 2009a. Seasonal monogamy and multiple paternity in a wild population of a territorial reptile (tuatara). Biological Journal of the Linnean Society 98:161-170.

Moore JA, Daugherty CH, Nelson NJ. 2009b. Large male advantage: Phenotypic and genetic correlates of territoriality in tuatara. Journal of Herpetology 43:570-578.

Moore JA, Hoare JM, Daugherty CH, Nelson NJ. 2007. Waiting reveals waning weight: Monitoring over 54 years shows a decline in body condition of a long-lived reptile (tuatara, Sphenodon punctatus). Biological Conservation 135:181-188.

Morris R. 1984. Developments of a water-maze procedure for studying spatial learning in the rat. Journal of Neuroscience Methods 11:47-60.

Morris RGM, Garrud P, Rawlins JNP, O’Keefe J. 1982. Place navigation impaired in rats 
with hippocampal lesions. Nature 297:681-683.

Moser E, Mathiesen I, Andersen P. 1993. Association between brain temperature and dentate field potentials in exploring and swimming rats. Science 259:1324-1326.

Moser MB, Trommald M, Andersen P. 1994. An increase in dendritic spine density on hippocampal CA1 pyramidal cells following spatial learning in adult rats suggests the formation of new synapses. Proceedings of the National Academy of Sciences 91:12673-12675.

Mullner P, Spencer SEF, Wilson DJ, Jones G, Noble AD, Midwinter AC, Collins-Emerson JM, Carter P, Hathaway S, French NP. 2009. Assigning the source of human campylobacteriosis in New Zealand: A comparative genetic and epidemiological approach. Infection, Genetics and Evolution 9:1311-1319.

Munnoch SA, Ward K, Sheridan S, Fitzsimmons GJ, Shadbolt CT, Piispanen JP, Wang Q, Ward TJ, Worgan TLM, Oxenford C. 2009. A multi-state outbreak of Salmonella Saintpaul in Australia associated with cantaloupe consumption. Epidemiology and Infection 137:367-374.

Nager RG, van Noordwijk AJ. 1995. Proximate and ultimate aspects of phenotypic plasticity in timing of great tit breeding in a heterogeneous environment. American Naturalist 156:454-474.

Navarro C, Marzal A, De Lope F, Møller AP. 2003. Dynamics of an immune response in house sparrows Passer domesticus in relation to time of day, body condition and blood parasite infection. Oikos 101:291-298.

Nealis VG, Jones RE, Wellington WG. 1984. Temperature and development in hostparasite relationships. Oecologia 61:224-229.

Needham GR, Teel PD. 1991. Off-host physiological ecology of ixodid ticks. Annual Review of Entomology 36:659-681.

Nelson NJ. 1998. Conservation of Brothers Island tuatara (Sphenodon guntheri). Unpublished PhD thesis. Victoria University of Wellington, Wellington, New Zealand. Nelson NJ, Keall SN, Pledger S, Daugherty CH. 2002a. Male-biased sex ratio in a small tuatara population. Journal of Biogeography 29:633-640.

Nelson NJ, Keall SN, Brown D, Daugherty CH. 2002b. Establishing a new wild population of tuatara (Sphenodon guntheri). Conservation Biology 16:887-894.

Nelson NJ, Cree A, Thompson M, Keall SN, Daugherty CH. 2004a. Temperature-dependent 
sex determination in tuatara. Pages $53-58$ in N. Valenzuela and V. Lance, editors. Temperature-Dependent Sex Determination in Vertebrates. Smithsonian Institution Press, Washington, DC, USA.

Nelson NJ, Thompson MB, Pledger S, Keall SN, Daugherty CH. 2004b. Do TSD, sex ratios, and nest characteristics influence the vulnerability of tuatara to global warming? International Congress Series 1275:250-257.

Nelson NJ, Thompson MB, Pledger S, Keall SN, Daugherty CH. 2006. Performance of juvenile tuatara depends on age, clutch, and incubation regime. Journal of Herpetology 40:399-403.

Nelson NJ, Keall SN, Gaze P, Daugherty CH. 2008. Re-introduction of tuatara as part of an ecological restoration project on Wakatere-papanui Island, Marlborough Sounds, New Zealand. Pages 58-61 in P.S. Soorae, editor. Global re-introduction perspectives: re-introduction case-studies from around the globe. Gland, Switzerland: IUCN/SSC Reintroduction Specialist Group and Abu Dhabi, UAE: Environment Agency- Abu Dhabi.

New TR. 2008. Insect conservation on islands: setting the scene and defining the needs. Journal of Insect Conservation 12:197-204.

Newman DG. 1982. Tuatara, Sphenodon punctatus, and burrows, Stephens Island. Pages 213-221 in D.G. Newman, editor. New Zealand Herpetology. New Zealand Wildlife Service, Dept. of Internal Affairs, New Zealand.

Newman DG. 1987. Burrow use and population densities of tuatara (Sphenodon punctatus) and how they are influenced by fairy prions (Pachyptila turtur) on Stephens Island, New Zealand. Herpetologica 43:336-344.

Newman DG, Watson PR, McFadden I. 1994. Egg production by tuatara on Lady Alice and Stephens Island, New Zealand. New Zealand Journal of Zoology 21:387-398.

Newman DG. 1998. Minutes of the Tuatara Recovery Group meeting held on Matiu/Somes Island, 4-5 March 1998. Unpublished report, Department of Conservation, Wellington.

Nichols WJ, Resendiz A, Seminoff JA, Resendiz B. 2000. Transpacific migration of a loggerhead turtle monitored by satellite telemetry. Bulletin of Marine Science 67:937-947.

Noble DWA, Byrne RW, Whiting MJ. 2014. Age-dependent social learning in a lizard. 
Biology Letters 10:20140430.

Noble DWA, Carazo P, Whiting MJ. 2012. Learning outdoors: male lizards show flexible spatial learning under semi-natural conditions. Biology Letters 8:946-948.

Northcutt GR, Heath JE. 1971. Performance of caimans in a T-maze. Copeia 1971:557560.

Northcutt RG, Heath JE. 1973. T-Maze behavior of the tuatara (Sphenodon punctatus). Copeia 1973:617-620.

Nussear KE, Tracy CR, Medica PA, Wilson DS, Marlow RW, Corn PS. 2012. Translocation as a conservation tool for Agassiz's desert tortoises: Survivorship, reproduction, and movements. The Journal of Wildlife Management 76: 1341-1353.

Nussey DH, Wilson AJ, Brommer JE. 2007. The evolutionary ecology of individual phenotypic plasticity in wild populations. Journal of Evolutionary Biology 20:831844.

O'Brien SJ. 2000. Adaptive cycles: parasites selectively reduce inbreeding in Soay sheep. Trends in Ecology \& Evolution 15:7-9.

Odling-Smee L, Braithwaite VA. 2003. The influence of habitat stability on landmark use during spatial learning in the three-spined stickleback. Animal Behaviour 65:701707.

Ogden NH, Lindsay LR, Beauchamp G, Charron D, Maarouf A, O'Callaghan CJ, WaltnerToews D, Barker IK. 2004. Investigation of relationships between temperature and developmental rates of tick Ixodes scapularis (Acari: Ixodidae) in the laboratory and field. Journal of Medical Entomology 41:622-633.

Oliver JH. 1989. Biology and systematics of ticks (Acari: Ixodida). Annual Review of Ecology and Systematics 20:397-430.

On SLW, Brandt SM, Cornelius AJ, Fusco V, Quero GM, Maćkiw E, Houf K, Bilbao A, Díaz Al, Benejat L. 2013. PCR revisited: a case for revalidation of PCR assays for microorganisms using identification of Campylobacter species as an exemplar. Quality Assurance and Safety of Crops \& Foods 5:49-62.

Oppliger A, Clobert J, Lecomte J, Lorenzon P, Boudjemadi K, John-Alder HB. 1998. Environmental stress increases the prevalence and intensity of blood parasite infection in the common lizard Lacerta vivipara. Ecology Letters 1:129-138.

Orr HA. 2009. Fitness and its role in evolutionary genetics. Nature Reviews Genetics 
10:531-539.

Otokunefor TV, Kindzeka BI, Ibiteye IO, Osuji GU, Obi FO, Jack AWK. 2003. Salmonella in gut and droppings of three pest lizards in Nigeria. World Journal of Microbiology and Biotechnology 19:545-548.

Owen K. 1998. Introduction of northern tuatara to Moutohora Island, Bay of Plenty. Ecological Management 6:23-33.

Owens D, Commuzzie DC, Grassman M. 1986. Chemoreception in the homing and orientation behavior of amphibians and reptiles, with special reference to sea turtles. Pages 341-355 in D. Duvall, D. Müller-Schwarze, and R. Silverstein, editors. Chemical Signals in Vertebrates 4. Plenum Press, New York, USA.

Palmer M, Dixon P. 1990. Small-scale environmental heterogeneity and the analysis of species distributions along gradients. Journal of Vegetation Science 1:57-65.

Paradis S, Cabanac M. 2004. Flavor aversion learning induced by lithium chloride in reptiles but not in amphibians. Behavioural Processes 67:11-18.

Parmesan C. 2006. Ecological and evolutionary responses to recent climate change. Review of Ecological and Evolutionary Systems 37:57-65.

Parmesan C. 2007. Influences of species, latitudes and methodologies on estimates of phenological response to global warming. Global Change Biology 13:1860-1872.

Parsons SK, Bull CM, Gordon DM. 2010. Low prevalence of Salmonella enterica in Australian wildlife. Environmental Microbiology Reports 2:657-659.

Pasmans F, Van Immerseel F, Van Den Broeck W, Bottreau E, Velge P, Ducatelle R, Haesebrouck F. 2003. Interactions of Salmonella enterica subsp. enterica serovar Muenchen with intestinal explants of the turtle Trachemys scripta scripta. Journal of Comparative Pathology 128:119-126.

Paterson AM, Palma RL, Gray RD. 2003. Drowning on arrival, missing the boat, and $x-$ events: How likely are sorting events? Pages 287-309 in R.D.M. Page, editor. Tangled trees: Phylogeny, cospeciation, and coevolution. University of Chicago Press, Chicago, USA.

Patz JA, Reisen WK. 2001. Immunology, climate change and vector-borne diseases. Trends in Immunology 22:171-172.

Paulissen MA. 2008. Spatial learning in the little brown skink, Scincella lateralis: the importance of experience. Animal Behaviour 76:135-141. 
Penafiel A, Rivera A, Gutierrez A, Trias S, De La Calle A. 2001. Temperature affects adult neurogenesis in the lizard brain. International Journal of Developmental Biology 45:S83-S84.

Pepperberg IM, Sandefer RM, Noel DA, Ellsworth CP. 2000. Vocal learning in the grey parrot (Psittacus erithacus): Effects of species identity and number of trainers. Comparative Psychology 114:371-380.

Perring TM, Holtzer TO, Toole JL, Norman JM, Myers GL. 1984. Influences of temperature and humidity on pre-adult development of the Banks grass mite (Acari: Tetranychidae). Environmental Entomology 13:338-343.

Petney TN, Andrews RH, Bull CM. 1983. Movement and host finding by unfed nymphs of two Australian reptile ticks. Australian Journal of Zoology 31:717-721.

Petranka JW, Sih A. 1986. Environmental instability, competition, and density-dependent growth and survivorship of a stream-dwelling salamander. Ecology 67:729-736.

Pfleger S, Benyr G, Sommer R, Hassl A. 2003. Pattern of Salmonella excretion in amphibians and reptiles in a vivarium. International Journal of Hygiene and Environmental Health 206:53-59.

Pianka ER, Pianka HD. 1970. The ecology of Moloch horridus (Lacertilia: Agamidae) in western Australia. Copeia:90-103.

Piersma T, Lindström Å. 1997. Rapid reversible changes in organ size as a component of adaptive behaviour. Trends in Ecology \& Evolution 12:134-138.

Pigliucci M. 2001. Environmental heterogeneity: temporal and spatial. Encyclopaedia of Life Sciences.

Pinter-Wollman N, Isbell LA, Hart LA. 2009. Assessing translocation outcome: Comparing behavioral and physiological aspects of translocated and resident African elephants (Loxodonta africana). Biological Conservation 142:1116-1124.

Pinter A, Dias RA, Gennari SM, Labruna MB. 2004. Study of the seasonal dynamics, life cycle, and host specificity of Amblyomma aureolatum (Acari: Ixodidae). Journal of Medical Entomology 41:324-332.

Polley L, Thompson RCA. 2009. Parasite zoonoses and climate change: molecular tools for tracking shifting boundaries. Trends in Parasitology 25:285-291.

Pörtner HO. 2002. Physiological basis of temperature-dependent biogeography: tradeoffs in muscle design and performance in polar ectotherms. Journal of Experimental 
Biology 205:2217-2230.

Poulin R. 1999. The functional importance of parasites in animal communities: many roles at many levels? International Journal for Parasitology 29:903-914.

Poulin R. 2011. Evolutionary ecology of parasites. Princeton University Press, Princeton, USA.

Pounds JA, Bustamante MR, Coloma LA, Consuegra JA, Fogden MPL, Foster PN, La Marca E, Masters KL, Merino-Viteri A, Puschendorf R. 2006. Widespread amphibian extinctions from epidemic disease driven by global warming. Nature 439:161-167.

Pounds JA, Fogden MPL, Campbell JH. 1999. Biological response to climate change on a tropical mountain. Nature 398:611-615.

Punzo F. 2002. Spatial associative learning in the crevice spiny lizard, Sceloporus poinsettii (Sauria: Iguanidae). The Texas Journal of Science 54:45-50.

Qin J, Wheeler AR. 2007. Maze exploration and learning in C. elegans. Lab on a Chip 7:186192.

Quallo MM, Price CJ, Ueno K, Asamizuya T, Cheng K, Lemon RN, Iriki A. 2009. Gray and white matter changes associated with tool-use learning in macaque monkeys. Proceedings of the National Academy of Sciences $106: 18379-18384$.

Quintero I, Wiens JJ. 2013. Rates of projected climate change dramatically exceed past rates of climatic niche evolution among vertebrate species. Ecology Letters 16:10951103.

R Core Team. 2015. R: A language and environment for statistical computing. $\mathrm{R}$ Foundation for Statistical Computing, Vienna, Austria. Available from https://www.rproject.org/.

Rabsch W, Fruth A, Simon S, Szabo I, Malorny B. 2015. The zoonotic agent Salmonella. Pages 179-211 in A. Sing, editor. Zoonoses-Infections affecting humans and animals. Springer Netherlands, Heidelberg, Germany.

Radmilovich M, Fernández A, Trujillo-Cenóz O. 2003. Environment temperature affects cell proliferation in the spinal cord and brain of juvenile turtles. Journal of Experimental Biology 206:3085-3093.

Ramsey JD, Dayal D, Ghahramani B. 1975. Heat stress limits for the sedentary worker. The American Industrial Hygiene Association Journal 36:259-265.

Ramsey JD, Kwon YG. 1992. Recommended alert limits for perceptual motor loss in hot 
environments. International Journal of Industrial Ergonomics 9:245-257.

Randolph SE. 2004. Evidence that climate change has caused "emergence" of tick-borne diseases in Europe? International Journal of Medical Microbiology Supplements 293:5-15.

Réale D, McAdam AG, Boutin S, Berteaux D. 2003. Genetic and plastic responses of a northern mammal to climate change. Proceedings of the Royal Society of London B: Biological Sciences 270:591-596.

Refsnider J. 2013. High thermal variance in naturally incubated turtle nests produces faster offspring. Journal of Ethology 31:85-93.

Refsnider JM, Janzen FJ. 2012. Behavioural plasticity may compensate for climate change in a long-lived reptile with temperature-dependent sex determination. Biological Conservation 152:90-95.

Reisinger A, Kitching R, Chiew F, Hughes L, Newton P, Schuster S, Tait A, Whetton P. 2014. Australasia. Pages 1371-1438 in V.R. Barros, C.B. Field, D.J. Dokken, M.D. Mastrandrea, K.J. Mach, T.E. Bilir, M. Chatterjee, K.L. Ebi, Y.O. Estrada, R.C. Genova, B. Girma, E.S. Kissel, A.N. Levy, S. MacCracken, P.R. Mastrandrea, L.L. White, editors. Climate Change 2014: Impacts, Adaptation, and Vulnerability. Part B: Regional Aspects. Contribution of Working Group II to the Fifth Assessment Report of the Intergovernmental Panel on Climate Change. Cambridge University Press, Cambridge, UK and New York, USA.

Reisinger LS, Petersen I, Hing JS, Davila RL, Lodge DM. 2015. Infection with a trematode parasite differentially alters competitive interactions and antipredator behaviour in native and invasive crayfish. Freshwater Biology 60:1581-1595.

Renwick AR, Lambin X. 2013. Host-parasite interactions in a fragmented landscape. International Journal for Parasitology 43:27-35.

Richter S, Kipfer T, Wohlgemuth T, Guerrero CC, Ghazoul J, Moser B. 2012. Phenotypic plasticity facilitates resistance to climate change in a highly variable environment. Oecologia 169:269-279.

Rissman EF, Heck AL, Leonard JE, Shupnik MA, Gustafsson JÅ. 2002. Disruption of estrogen receptor $\beta$ gene impairs spatial learning in female mice. Proceedings of the National Academy of Sciences 99:3996-4001.

Robb J. 1977. The tuatara. Meadowfield Press, Shildon, UK. 
Robertson RM. 2004a. Modulation of neural circuit operation by prior environmental stress. Integrative and Comparative Biology 44:21-27.

Robertson RM. 2004b. Thermal stress and neural function: adaptive mechanisms in insect model systems. Journal of Thermal Biology 29:351-358.

Rodda G. 1984. The orientation and navigation of juvenile alligators: evidence of magnetic sensitivity. Journal of Comparative Physiology A 154:649-658.

Rodgers SE, Zolnik CP, Mather TN. 2007. Duration of exposure to suboptimal atmospheric moisture affects nymphal blacklegged tick survival. Journal of Medical Entomology 44:372-375.

Rodríguez-Prieto I, Martín J, Fernández-Juricic E. 2010. Individual variation in behavioural plasticity: direct and indirect effects of boldness, exploration and sociability on habituation to predators in lizards. Proceedings of the Royal Society of London B: Biological Sciences 278:266-273.

Rodríguez F, López JC, Vargas P, Gómez Y, Broglio C, Salas C. 2002. Conservation of spatial memory function in the pallial forebrain of reptiles and ray-finned fishes. The Journal of Neuroscience 22:2894-2903.

Romano JC, Bensoussan N, Younes WA, Arlhac D. 2000. Thermal anomaly in waters of the Gulf of Marseilles during the summer of 1999. A partial explanation of the mortality of certain fixed invertebrates? Comptes rendus de l'Academie des sciences. Serie III, Sciences de la vie 323:415-427.

Romijn RL, Hartley S. 2016. Trends in lizard translocations in New Zealand between 1988 and 2013. New Zealand Journal of Zoology 43:191-210.

Root TL, Price JT, Hall KR, Schneider SH, Rosenzweig C, Pounds JA. 2003. Fingerprints of global warming on wild animals and plants. Nature 421:57-60.

Roth ED, Lutterschmidt WI, Wilson DA. 2006. Relative medial and dorsal cortex volume in relation to sex differences in spatial ecology of a snake population. Brain, Behavior and Evolution 67:103-110.

Rowe C, Healy SD. 2014. Measuring variation in cognition. Behavioral Ecology:aru090.

Russell A, Bauer A, Johnson M. 2005. Migration in amphibians and reptiles: An overview of patterns and orientation mechanisms in relation to life history strategies. Pages 151-203 in A.T. Elewa, editor. Migration of Organisms. Springer-Verlag, Berlin, Germany. 
Sabat P, Bozinovic F. 2000. Digestive plasticity and the cost of acclimation to dietary chemistry in the omnivorous leaf-eared mouse Phyllotis darwini. Journal of Comparative Physiology B 170:411-417.

Saint Girons H. 1980. Thermoregulation in reptiles with special reference to the tuatara and its ecophysiology. Tuatara 24:59-77.

Saint Girons H, Bell BD, Newman DG. 1980. Observations on the activity and thermoregulation of the tuatara, Sphenodon punctatus (Reptilia: Rhynchocephalia), on Stephens Island. New Zealand Journal of Zoology 7:551-556.

Samour HJ, Spratt DMJ, Hart MG, Savage B, Hawkey CM. 1987. A survey of the Aldabra giant tortoise population introduced on Curieuse Island, Seychelles. Biological conservation 41:147-158.

Sanz VGA. 1998. Successful Reintroduction of Captive-Raised Yellow-Shouldered Amazon Parrots on Margarita Island, Venezuela. Conservation Biology 12:430.

Sasa M. 1961. Biology of chiggers. Annual Review of Entomology 6:221-244.

Sauer JR, Hair JA. 1971. Water balance in the Lone Star tick (Acarina: Ixodidae): the effects of relative humidity and temperature on weight changes and total water content. Journal of Medical Entomology 8:479-485.

Savill MG, Hudson JA, Ball A, Klena JD, Scholes P, Whyte RJ, McCormick RE, Jankovic D. 2001. Enumeration of Campylobacter in New Zealand recreational and drinking waters. Journal of Applied Microbiology 91:38-46.

Sawyer SL, Fogle SR. 2010. Acoustic attraction of grey-faced petrels (Pterodroma macroptera gouldi) and fluttering shearwaters (Puffinus gavia) to Young Nick's Head, New Zealand. Notornis 57:166-168.

Schall JJ. 2000. Learning in free-ranging populations of the whiptail lizard Cnemidophorus murinus. Herpetologica 56:38-45.

Scheelings TF, Lightfoot D, Holz P. 2011. Prevalence of Salmonella in Australian reptiles. Journal of Wildlife Diseases 47:1-11.

Schenk F, Morris RGM. 1985. Dissociation between components of spatial memory in rats after recovery from the effects of retrohippocampal lesions. Experimental Brain Research 58:11-28.

Schiff SJ, Somjen GG. 1985. The effects of temperature on synaptic transmission in hippocampal tissue slices. Brain Research 345:279-284. 
Scott ME. 1988. The impact of infection and disease on animal populations: Implications for conservation biology. Conservation Biology 2:40-56.

Sears A, Baker MG, Wilson N, Marshall J, Muellner P, Campbell DM, Lake RJ, French NP. 2011. Marked campylobacteriosis decline after interventions aimed at poultry, New Zealand. Emerging Infectious Diseases 17:1007-1015.

Seddon PJ. 1999. Persistence without intervention: assessing success in wildlife reintroductions. Trends in Ecology \& Evolution 14:503.

Seddon PJ, Soorae PS, Launay F. 2005. Taxonomic bias in reintroduction projects. Animal Conservation 8:51-58.

Seddon PJ, Strauss WM, Innes J. 2012. Animal translocations: what are they and why do we do them? In J. Ewen, D. Armstrong, K. Parker, and P. Seddon, editors. Reintroduction Biology: Integrating Sceince and Management. Wiley Blackwell, Oxford, UK.

Seebacher F. 1999. Behavioural postures and the rate of body temperature change in wild freshwater crocodiles, Crocodylus johnstoni. Physiological and Biochemical Zoology 72:57-63.

Seebacher F. 2005. A review of thermoregulation and physiological performance in reptiles: what is the role of phenotypic flexibility? Journal of Comparative Physiology B 175:453-461.

Seigel RA, Dodd CK. 2002. Translocations of amphibians: proven management method or experimental technique? Conservation Biology 16:552-554.

Seigel RA, Ford NB. 1991. Phenotypic plasticity in the reproductive characteristics of an oviparous snake, Elaphe guttata: implications for life history studies. Herpetologica 47:301-307.

Sherry DF, Forbes MR, Khurgel M, Ivy GO. 1993. Females have a larger hippocampus than males in the brood-parasitic brown-headed cowbird. Proceedings of the National Academy of Sciences $90: 7839-7843$.

Sherry DF, Vaccarino AL. 1989. Hippocampus and memory for food caches in black-capped chickadees. Behavioral Neuroscience 103:308-318.

Shettleworth SJ. 2010. Cognition, Evolution and Behavior, 2nd edition. Oxford University Press, New York, USA.

Shibasaki K, Suzuki M, Mizuno A, Tominaga M. 2007. Effects of body temperature on 
neural activity in the hippocampus: regulation of resting membrane potentials by transient receptor potential vanilloid 4. The Journal of Neuroscience 27:1566-1575. Short J. 2009. The characteristics and success of vertebrate translocations within Australia. Unpublished progress report to the Department of Agriculture, Fisheries and Forestry, Australia.

Silva AJ, Paylor R, Wehner JM, Tonegawa S. 1992. Impaired spatial learning in alphacalcium-calmodulin kinase II mutant mice. Science 257:206-211.

Simmons G, Hope V, Lewis G, Whitmore J, Gao W. 2001. Contamination of potable roofcollected rainwater in Auckland, New Zealand. Water Research 35:1518-1524.

Sloss MW, Kemp RL, Zajac AM. 1994. Veterinary clinical parasitology. Iowa State University Press, lowa City, USA.

Smith TG. 1996. The genus Hepatozoon (Apicomplexa: Adeleina). The Journal of Parasitology 82:565-585.

Somero GN. 1995. Proteins and temperature. Annual Review of Physiology 57:43-68.

Somero GN, Dahlhoff E, Lin JJ. 1996. Stenotherms and eurytherms: mechanisms establishing thermal optima and tolerance ranges. Pages 53-78 in I.A. Johnston and A.F. Bennett, editors. Animals and temperature. Phenotypic and Evolutionary Adaptation. Cambridge University Press, New York, USA.

Somero GN, DeVries AL. 1967. Temperature tolerance of some Antarctic fishes. Science 156:257-258. American Association for the Advancement of Science.

Soorae PS, editor. 2010. Global Re-introduction Perspectives: Additional case studies from around the globe. Gland, Switzerland: IUCN/SSC Reintroduction Specialist Group and Abu Dhabi, UAE: Environment Agency- Abu Dhabi.

Soorae PS, editor. 2011. Global Re-introduction Perspectives: 2011: More case studies from around the globe. Gland, Switzerland: IUCN/SSC Reintroduction Specialist Group and Abu Dhabi, UAE: Environment Agency- Abu Dhabi.

Soorae PS, editor. 2013. Global Re-introduction Perspectives: 2013. Further case studies from around the globe. Gland, Switzerland: IUCN/SSC Reintroduction Specialist Group and Abu Dhabi, UAE: Environment Agency- Abu Dhabi.

Spalton JA, Lawrence MW, Brend SA. 1999. Arabian oryx reintroduction in Oman: successes and setbacks. Oryx 33:168-175.

Stafford KC. 1994. Survival of immature Ixodes scapularis (Acari: Ixodidae) at different 
relative humidities. Journal of Medical Entomology 31:310-314.

Stamps JA. 1994. Territorial behavior: testing the assumptions. Advances in the Study of Behavior 23:173-232.

Stamps JA, Krishnan VV. 1998. Territory acquisition in lizards. IV. Obtaining high status and exclusive home ranges. Animal Behaviour 55:461-472.

Stein RA, Magnuson JJ. 1976. Behavioral Response of crayfish to a fish predator. Ecology 57:751-761.

Stein RB, Gordon T, Shriver J. 1982. Temperature dependence of mammalian muscle contractions and ATPase activities. Biophysical Journal 40:97-107.

Stevenson M. 2014. epiR: an R package for the analysis of epidemiological data.

Stevenson RD. 1985a. The relative importance of behavioral and physiological adjustments controlling body temperature in terrestrial ectotherms. American Naturalist 126:362-386.

Stevenson RD. 1985b. Body size and limits to the daily range of body temperature in terrestrial ectotherms. American Naturalist:102-117.

Stoddart DR, Cowx D, Peet C, Wilson JR. 1982. Tortoises and tourists in the western Indian Ocean: the Curieuse experiment. Biological Conservation 24:67-80.

Stone A, Ford NB, Holtzman DA. 2000. Spatial learning and shelter selection by juvenile spotted pythons, Anteresia maculosus. Journal of Herpetology 34:575-587.

Sun D, Niu C. 2012. Adaptive significance of temperature-induced egg size plasticity in a planktonic rotifer, Brachionus calyciflorus. Journal of Plankton Research 34:864-873.

Sutherland RJ, Kolb B, Whishaw IQ. 1982. Spatial mapping: definitive disruption by hippocampal or medial frontal cortical damage in the rat. Neuroscience Letters 31:271-276.

Tajkarimi M. 2007. Salmonella spp. Available from https://www.cdfa.ca.gov/ ahfss/Animal_Health/PHR250/2007/25007Sal.pdf (accessed September 20, 2015)

Tamme R, Hiiesalu I, Lannisto L, Szava-Kovats R, Partel M. 2010. Environmental heterogeneity, species diversity and co-existence at different spatial scales. Journal of Vegetation Science 21:796-801.

Taylor E, Kastner J, Renter D. 2010. Challenges involved in the Salmonella Saintpaul outbreak and lessons learned. Journal of Public Health Management and Practice $16: 221-231$. 
Teixeira CP, De Azevedo CS, Mendl M, Cipreste CF, Young RJ. 2007. Revisiting translocation and reintroduction programmes: the importance of considering stress. Animal Behaviour 73:1-13.

Terezow MG, Nelson NJ, Markwell TJ. 2008. Circadian emergence and movement of captive juvenile tuatara (Sphenodon spp.). New Zealand Journal of Zoology 35:205216.

Thomas CD. 2011. Translocation of species, climate change, and the end of trying to recreate past ecological communities. Trends in Ecology \& Evolution 26:216-221.

Thomas JA, Simcox DJ, Clarke RT. 2009. Successful conservation of a threatened Maculinea butterfly. Science 325:80-83.

Thomason BM, Dodd DJ, Cherry WB. 1977. Increased recovery of Salmonellae from environmental samples enriched with buffered peptone water. Applied and Environmental Microbiology 34:270-273.

Thompson MB. 1990. Incubation of eggs of tuatara, Sphenodon punctatus. Journal of Zoology 222:303-318.

Thompson MB, Packard GC, Packard MJ, Rose B. 1996. Analysis of the nest environment of tuatara Sphenodon punctatus. Journal of Zoology 238:239-251.

Thompson MB, Daugherty CH. 1998. Metabolism of tuatara, Sphenodon punctatus. Comparative Biochemistry and Physiology Part A: Molecular \& Integrative Physiology 119:519-522.

Thompson RCA, Lymbery AJ, Smith A. 2010. Parasites, emerging disease and wildlife conservation. International Journal for Parasitology 40:1163-1170.

Thorsen M. 2004. Whangeokena (East Island) ecological restoration plan. Technical Support Series No. 16. Gisborne, New Zealand.

Tinklepaugh OL. 1932. Maze learning of a turtle. Journal of Comparative Psychology 13:201-206.

Tizard I. 2004. Salmonellosis in wild birds. Pages 50-66 Seminars in Avian and Exotic Pet Medicine 13:50-66.

Tolson PJ, García MA, Pierce JJ. 2008. Re-introduction of the Virgin Islands boa to the Puerto Rico Bank. Pages 66-69 in P.S. Soorae, editor. Global re-introduction perspectives: re-introduction case-studies from around the globe. Gland, Switzerland: IUCN/SSC Reintroduction Specialist Group and Abu Dhabi, UAE: 
Environment Agency- Abu Dhabi.

Tompkins DM, Dunn AM, Smith MJ, Telfer S. 2011. Wildlife diseases: from individuals to ecosystems. Journal of Animal Ecology 80:19-38.

Torchin ME, Lafferty KD, Dobson AP, McKenzie VJ, Kuris AM. 2003. Introduced species and their missing parasites. Nature 421:628-630.

Torson AS, Yocum GD, Rinehart JP, Kemp WP, Bowsher JH. 2015. Transcriptional responses to fluctuating thermal regimes underpinning differences in survival in the solitary bee Megachile rotundata. Journal of Experimental Biology 218:1060-1068.

Towns DR, Daugherty CH, Cree A. 2001. Raising the prospects for a forgotten fauna: a review of 10 years of conservation effort for New Zealand reptiles. Biological Conservation 99:3-16.

Towns DR, Ferreira SM. 2001. Conservation of New Zealand lizards (Lacertilia: Scincidae) by translocation of small populations. Biological Conservation 98:211-222.

Towns DR, Parrish GR, Tyrrell CL, Ussher GT, Cree A, Newman DG, Whitaker AH, Westbrooke I. 2007. Responses of tuatara (Sphenodon punctatus) to removal of introduced pacific rats from islands. Conservation Biology 21:1021-1031.

Travis JMJ. 2003. Climate change and habitat destruction: a deadly anthropogenic cocktail. Proceedings of the Royal Society of London B: Biological Sciences 270:467473.

Tu Z, Zeitlin G, Gagner J, Keo T, Hanna BA, Blaser MJ. 2004. Campylobacter fetus of reptile origin as a human pathogen. Journal of Clinical Microbiology 42:4405.

Ueda S, Ibuka N. 1995. An analysis of factors that induce hibernation in Syrian hamsters. Physiology \& Behavior 58:653-657.

Ujvari B, Madsen T. 2006. Age, parasites, and condition affect humoral immune response in tropical pythons. Behavioral Ecology 17:20-24.

Urban MC, Richardson JL, Freidenfelds NA. 2014. Plasticity and genetic adaptation mediate amphibian and reptile responses to climate change. Evolutionary Applications 7:88-103.

US Fish And Wildlife Service. 2008. Rocky Mountain wolf recovery 2008 interagency annual report. USFWS, Ecological Services, Helena, Montana, USA.

Ussher GT. 2003. Proposal to translocate northern tuatara (Sphenodon punctatus punctatus) from Middle Island, Mercury Group, to Tiritiri Matangi Island, north 
Auckland, in mid-October 2003. Unpublished report to Department of Conservation, Auckland, New Zealand.

Valenzuela N, Lance V, editors. 2004. Temperature-dependent sex determination in vertebrates. Smithsonian Institution Press, Washington, DC, USA.

van Andel M, Jackson BH, Midwinter AC, Alley MR, Ewen JG, Mclnnes K, Jakob Hoff R, Reynolds AD, French N. 2014. Investigation of mortalities associated with Salmonella spp. infection in wildlife on Tiritiri Matangi Island in the Hauraki Gulf of New Zealand. New Zealand Veterinary Journal 63:235-239.

van Winkel D, Baling M, Barry M, Weihong J, Brunton D. 2010. Translocation of Duvaucel's geckos to Tiritiri Matangi and Motuora Islands, Hauraki Gulf, as part of island ecological restoration initiatives. Pages 113-117 in P.S. Soorae, editor. Global reintroduction perspectives: Additional case-studies from around the globe. Gland, Switzerland: IUCN/SSC Reintroduction Specialist Group and Abu Dhabi, UAE: Environment Agency- Abu Dhabi.

Vermunt A, Hare KM, Besson AA. 2014. Unusual change in activity pattern at cool temperature in a reptile (Sphenodon punctatus). Journal of Thermal Biology 42:4045.

Visser ME. 2008. Keeping up with a warming world; assessing the rate of adaptation to climate change. Proceedings of the Royal Society B: Biological Sciences 275:649-659.

Vitt L, Caldwell JP. 2009. Herpetology, 3rd edition. Academic Press/Elsevier, San Diego, USA.

Waite DW, Deines P, Taylor MW. 2012. Gut microbiome of the critically endangered New Zealand parrot, the kakapo (Strigops habroptilus). PLoS ONE 7:e35803.

Walls GY. 1978. The influence of the tuatara on fairy prion breeding on Stephens Island, Cook Strait. New Zealand Journal of Ecology 1:91-98.

Walls GY. 1980. Provisional results from a study of the feeding ecology of the tuatara (Sphenodon punctatus) on Stephens Island. In D.G. Newman, editor. New Zealand Herpetology. New Zealand Wildlife Service, Wellington, New Zealand

Walls GY. 1981. Feeding ecology of the tuatara (Sphenodon punctatus) on Stephens Island, Cook Strait. New Zealand Journal of Ecology 4:89-97.

Walls GY. 1983. Activity of the tuatara and its relationships to weather conditions on Stephens Island, Cook Strait, with observations on geckos and invertebrates. New 
Zealand Journal of Zoology 10:309-318.

Walther G, Post E, Convey P, Menzel A, Parmesan C, Beebee TJC, Fromentin J, HoeghGuldberg O, Bairlein F. 2012. Ecological responses to recent climate change. Nature 416:389-395.

Wang C, Shia W, Jhou Y, Shyu C. 2013. Occurrence and molecular characterization of reptilian Campylobacter fetus strains isolated in Taiwan. Veterinary Microbiology 164:67-76.

Wang Y, Guo F, Pan C, Lou Y, Zhang P, Guo S, Yin J, Deng Z. 2012. Effects of low temperatures on proliferation-related signaling pathways in the hippocampus after traumatic brain injury. Experimental Biology and Medicine 237:1424-1432.

Ward JR, Lafferty KD. 2004. The elusive baseline of marine disease: are diseases in ocean ecosystems increasing. PLOS Biology 2:e120.

Werner EE. 1986. Amphibian metamorphosis: growth rate, predation risk, and the optimal size at transformation. American Naturalist:319-341.

Werner YL, Whitaker AH. 1978. Observation and comments on the body temperatures of some New Zealand reptiles. New Zealand Journal of Zoology 5:375-393.

Wharton GW, Fuller HS. 1952. A manual of the chiggers. The biology, classification, distribution, and importance to man of the larvae of the family Trombiculidae (Acariña). Memoirs of the Entomology Society of Washington. Washington, DC, USA.

Whitman DW, Agrawal AA. 2009. What is phenotypic plasticity and why is it important? Pages 3-63 in D.W. Whitman and T.N. Ananthakrishnan, editors. Phenotypic plasticity of insects: mechanisms and consequences. Science Publishers, Enfield, New Hampshire, USA.

Wilbur HM. 1977. Density-dependent aspects of growth and metamorphosis in Bufo americanus. Ecology 58:196-200.

Wilkinson A, Huber L. 2012. Cold-blooded cognition: Reptilian cognitive abilities. Pages 129-143 in J. Vonk and T.K. Shackelford, editors. The Oxford Handbook of Comparative Evolutionary Psychology. Oxford University Press, New York, USA.

Wilkinson A, Kuenstner K, Mueller J, Huber L. 2010. Social learning in a non-social reptile (Geochelone carbonaria). Biology Letters:rsbl20100092.

Williams JW, Jackson ST, Kutzbach JE. 2007. Projected distributions of novel and disappearing climates by 2100 AD. Proceedings of the National Academy of Sciences 
104:5738-5742.

Wilson KJ, Lee AK. 1970. Changes in oxygen consumption and heart-rate with activity and body temperature in the tuatara, Sphenodon punctatum. Comparative Biochemistry and Physiology 33:311-322.

Windsor DA. 1998. Controversies in parasitology, most of the species on Earth are parasites. International Journal for Parasitology 28:1939-1941.

Winfield MD, Groisman EA. 2003. Role of nonhost environments in the lifestyles of Salmonella and Escherichia coli. Applied and Environmental Microbiology 69:36873694.

Wing JF. 1965. Upper thermal tolerance limits for unimpaired mental performance. Aerospace Medicine 36:960-964.

Wobeser G. 2002. Disease management strategies for wildlife. Revue Scientifique et Technique-Office international des epizooties 21:159-178.

Wolf CM, Griffith B, Reed C, Temple SA. 1996. Avian and mammalian translocations: update and reanalysis of 1987 survey data. Conservation Biology 10:1142-1154.

Woo KL, Hunt M, Harper D, Nelson NJ, Daugherty CH, Bell BD. 2009. Discrimination of flicker frequency rates in the reptile tuatara (Sphenodon). Naturwissenschaften 96:415-419.

Wood JR. 2009. Two late quaternary avifaunal assemblages from the Dunback district, eastern Otago, South Island, New Zealand. Notornis 56:154-157.

Woodford MH. 2000. Quarantine and health screening protocols for wildlife prior to translocation and release into the wild. IUCN Species Survival Commission's Veterinary Specialist Group, Gland, Switzerland, the Office International des Epizooties (OIE), Paris, France, Care for the Wild, U.K., and the European Association of Zoo and Wildlife Veterinarians, Switzerland.

Woodward DL, Khakhria R, Johnson WM. 1997. Human salmonellosis associated with exotic pets. Journal of Clinical Microbiology 35:2786-2790.

Worthy TH, Holdaway RN. 2002. The lost world of the moa: prehistoric life of New Zealand. Indiana University Press, Bloomington, USA.

Wu X, Jiang H. 2008. Re-introduction of Chinese alligators into the Gaojingmiao forestry farm, Anhui province, China. Pages $76-78$ in P.S. Soorae, editor. Global reintroduction perspectives: re-introduction case-studies from around the globe. 
Gland, Switzerland: IUCN/SSC Reintroduction Specialist Group and Abu Dhabi, UAE: Environment Agency- Abu Dhabi.

Yerkes RM. 1901. The formation of habits in the turtle. Popular Science Monthly 58.

Zamora-Vilchis I, Williams SE, Johnson CN. 2012. Environmental temperature affects prevalence of blood parasites of birds on an elevation gradient: implications for disease in a warming climate. PLoS ONE 7:e39208.

ZEALANDIA. 2011. ZEALANDIA, the Karori Sanctuary experience, te mara a Tane. Available from http://www.visitzealandia.com/what-is-zealandia/ (accessed November 30, 2015).

Zerbolio Jr. DJ. 1973. Temperature-dependent learning in goldfish: A multi-trial active avoidance situation. Behavioral Biology 8:755-761.

Zorn C. 2005. A solution to separation in binary response models. Political Analysis 13:157-170. 


\section{Appendices}

\section{Appendix 1}

Table A1.1 Information on the weather stations used to obtain regional temperature and relative humidity data (Figures 2 and 3 ). We selected stations in closest proximity to the study sites that had the required data type available. We used two different stations to obtain temperature and RH data for Maungatautari as RH data were not available from the Lake Karapiro Cws station, which was in closest proximity to the study site. Data were accessed through the NIWA Cliflo database (http://www.cliflo.niwa.co.nz). The Department of Conservation (DOC) staff collect weather station data for Stephens Island on behalf of the Metservice.

\begin{tabular}{|c|c|c|c|c|c|c|c|c|}
\hline Data type & Study site & Station name & $\begin{array}{c}\text { Agent } \\
\text { number }\end{array}$ & $\begin{array}{c}\text { Network } \\
\text { number }\end{array}$ & Latitude & Longitude & Height (m) & $\begin{array}{l}\text { Observing } \\
\text { authority }\end{array}$ \\
\hline \multirow{6}{*}{$\begin{array}{l}\text { Min, max \& } \\
\text { mean } \\
\text { temperature } \\
\left({ }^{\circ} \mathrm{C}\right)\end{array}$} & Cape Sanctuary & Cape Kidnappers Wxt Aws & 37870 & D97601 & -39.645 & 177.092 & 118 & Metservice \\
\hline & Maungatautari & Lake Karapiro Cws & 37656 & C75955 & -37.925 & 175.540 & 55 & NIWA \\
\hline & Stephens Island & Stephens Island & 4153 & G04601 & -40.666 & 174.001 & 187 & DOC \\
\hline & Whangaokeno & Hicks Bay Aws & 2692 & D78531 & -37.564 & 178.314 & 46 & Metservice \\
\hline & Young Nicks Head & Gisborne Aws & 2810 & D87695 & -38.660 & 177.984 & 5 & Metservice \\
\hline & ZEALANDIA & Wellington, Kelburn Aws & 25354 & E1427P & -41.285 & 174.768 & 125 & Metservice \\
\hline \multirow{7}{*}{$\begin{array}{l}\text { Relative } \\
\text { humidity } \\
\text { (\%) \& total } \\
\text { rainfall (mm) }\end{array}$} & \multirow{3}{*}{$\begin{array}{ll}\text { Cape Sanctuary } & \begin{array}{l}\mathrm{RH} \\
\text { rain }\end{array} \\
\text { Maungatautari } & \end{array}$} & Cape Kidnappers Wxt Aws & 37870 & D97601 & -39.645 & 177.092 & 118 & Metservice \\
\hline & & Hastings Aws & 3017 & D96680 & -39.648 & 176.841 & 16 & NA \\
\hline & & Hamilton Aws & 2112 & C75834 & -37.865 & 175.336 & 53 & Metservice \\
\hline & Stephens Island & Stephens Island & 4153 & G04601 & -40.666 & 174.001 & 187 & DOC \\
\hline & Whangaokeno & Hicks Bay Aws & 2692 & D78531 & -37.564 & 178.314 & 46 & Metservice \\
\hline & Young Nicks Head & Gisborne Aws & 2810 & D87695 & -38.660 & 177.984 & 5 & Metservice \\
\hline & ZEALANDIA & Wellington, Kelburn Aws & 25354 & E1427P & -41.285 & 174.768 & 125 & Metservice \\
\hline
\end{tabular}




\section{Appendix 2}
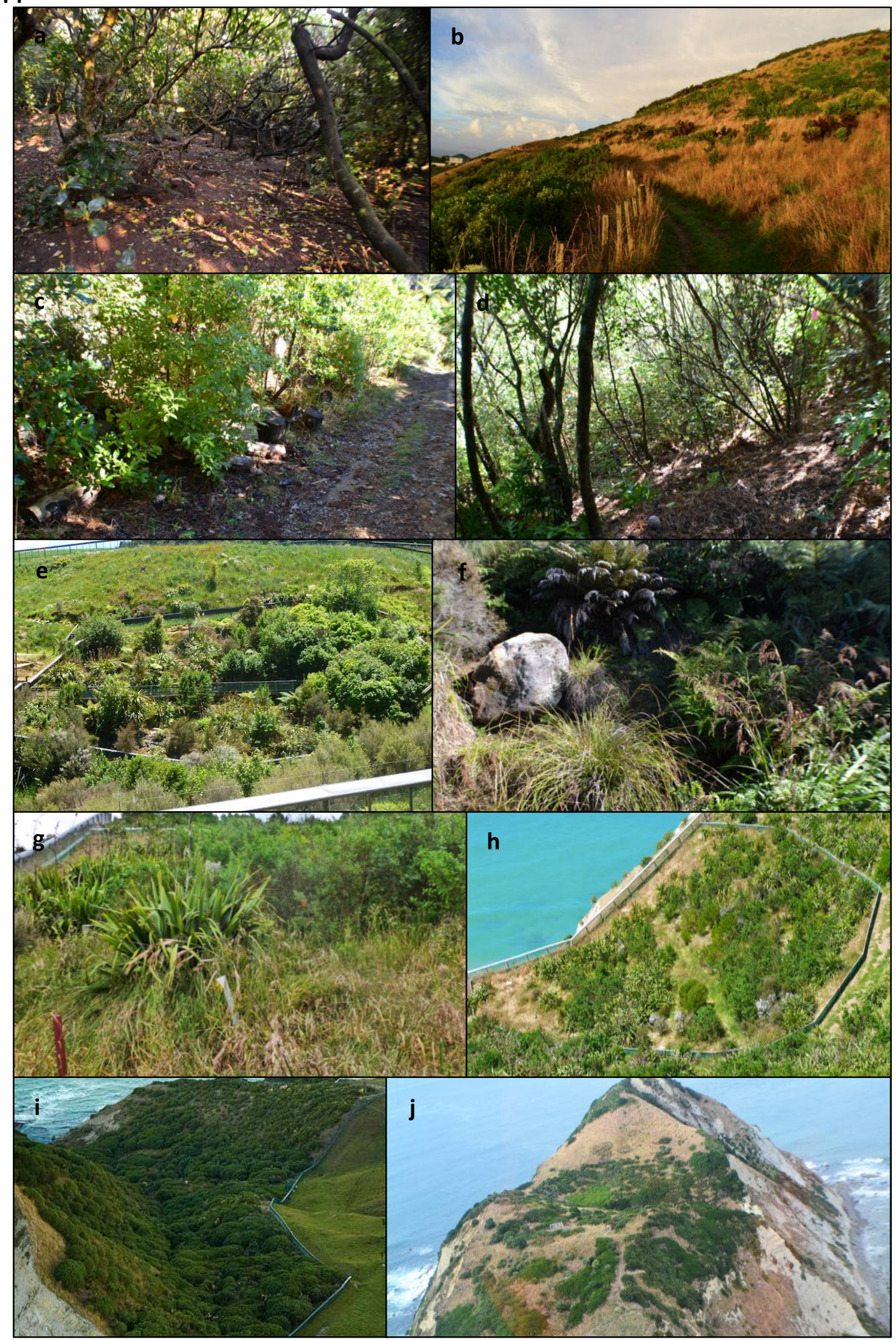

Figure A2.1 Photographs of study sites/habitats. a) Stephens Island coastal forest; b) Stephens Island grassland/shrubland; c) ZEALANDIA: habitat alongside a walking track; d) ZEALANDIA: forest habitat in the Research Area; e) Maungatautari Tautari Wetland tuatara enclosure; f) example habitat within the Tautari Wetland tuatara enclosure; g) example habitat in the Cape Sanctuary adult tuatara enclosure; h) looking down on the Cape Sanctuary adult tuatara enclosure from within the Seabird Cell; i) Young Nicks Head enclosure. Image: Marion Brenner; j) Whangaokeno taken from a helicopter. All images taken by S. Price (unless stated otherwise). 
Table A2.1 Table shows the number of adult animals released at each translocation site, SVL mean, minimum and maximum size (mm) on release in October 2012, and estimated SVL growth per month and per year. ZEALANDIA SVL on release data were calculated from animals released in 2005 and 2007 . SVL measurements on release were calculated from all adult tuatara released at each site, whereas estimated mean SVL growth per month and per year were calculated using data obtained from recaptured animals. Estimated SVL growth per year was calculated by multiplying monthly mean growth estimates by 12 . One female from Maungatautari and two females from ZEALANDIA were omitted from the analysis as their SVLs on release were $<170 \mathrm{~mm}$, classifying them as sub adult animals (Blanchard 2002).

\begin{tabular}{|c|c|c|c|c|c|c|c|}
\hline \multirow{2}{*}{ Site } & \multirow{2}{*}{ Sex } & \multirow{2}{*}{$\begin{array}{l}\text { No. } \\
\text { adults }\end{array}$} & \multicolumn{3}{|c|}{ SVL $(\mathrm{mm})$ on release } & \multirow{2}{*}{$\begin{array}{l}\text { SVL growth per } \\
\text { month }\left(\mathrm{mm} \mathrm{m}^{-1}\right)\end{array}$} & \multirow{2}{*}{$\begin{array}{l}\text { SVL growth per } \\
\text { year }\left(\mathrm{mm} \mathrm{y}^{-1}\right)\end{array}$} \\
\hline & & & Mean ( \pm SE) & Min. & Max. & & \\
\hline \multirow{2}{*}{ Cape Sanctuary } & Male & 20 & $228.45( \pm 4.44)$ & 173 & 259 & -0.021 & -0.252 \\
\hline & Female & 20 & $205.00( \pm 3.18)$ & 180 & 223 & -0.109 & -1.308 \\
\hline \multirow{2}{*}{ Maungatautari } & Male & 30 & $229.75( \pm 3.63)$ & 194 & 270 & 0.000 & 0.002 \\
\hline & Female & 19 & $199.54( \pm 3.27)$ & 175 & 233 & 0.001 & 0.008 \\
\hline \multirow{2}{*}{ Whangaokeno } & Male & 25 & $220.36( \pm 3.98)$ & 194 & 250 & 0.563 & 6.756 \\
\hline & Female & 19 & $186.92( \pm 3.28)$ & 174 & 209 & 0.364 & 4.368 \\
\hline \multirow{2}{*}{ Young Nicks Head } & Male & 23 & $230.83( \pm 4.16)$ & 179 & 263 & -0.007 & -0.084 \\
\hline & Female & 19 & $197.79( \pm 3.28)$ & 174 & 225 & -0.071 & -0.852 \\
\hline \multirow{2}{*}{ ZEALANDIA } & Male & 104 & $230.13( \pm 3.18)$ & 170 & 280 & 0.036 & 0.432 \\
\hline & Female & 94 & $194.56( \pm 1.96)$ & 172 & 280 & 0.047 & 0.570 \\
\hline
\end{tabular}


Table A2.2 Table showing result of LMMs run individually by sex to compare growth rates between recaptured Whangaokeno tuatara and tuatara from other translocation sites. The estimate shows the difference in monthly growth rate $(\mathrm{mm})$ between recaptured individuals from each named site and individuals from Whangaokeno. P-values indicate that all of these differences are statistically significant. Sample size shows the number of individuals recaptured at least once following release on which these analyses are based. Whangaokeno male sample size $=18$, female sample size $=5$.

\begin{tabular}{llrrrrr}
\hline \multicolumn{1}{c}{ Site } & \multicolumn{1}{c}{ Sex } & Sample size & Estimate & $\begin{array}{c}\text { Standard } \\
\text { error }\end{array}$ & T value & P value \\
\hline \multirow{2}{*}{ Cape Sanctuary } & Males & 18 & -0.582 & 0.139 & -4.184 & $<0.001$ \\
& Females & 19 & -0.478 & 0.144 & -3.314 & 0.001 \\
Maungatautari & Males & 21 & -0.562 & 0.135 & -4.156 & $<0.001$ \\
& Females & 10 & -0.364 & 0.151 & -2.411 & 0.018 \\
Young Nicks Head & Males & 5 & -0.571 & 0.185 & -3.089 & 0.003 \\
& Females & 2 & -0.437 & 0.213 & -2.052 & 0.044 \\
ZEALANDIA & Males & 21 & -0.528 & 0.116 & -4.555 & $<0.001$ \\
& Females & 20 & -0.318 & 0.136 & -2.347 & 0.022 \\
\hline
\end{tabular}




\section{Appendix 4}

Table A4.1 GLM outputs of best-fit models of predictors influencing tick load per seasonal survey. The levels of the factor site were re-levelled so that ZEALANDIA (the control site) was the baseline against which other sites were compared in each season. Further re-levelling of the baseline factor "site" for the autumn 2015 model confirmed that there is significant variation in tick load across multiple sites, though not all sites are significantly different to one another.

\begin{tabular}{|c|c|c|c|c|c|c|c|c|}
\hline Season/survey & Best-fit model & Model $\chi 2$ & $\begin{array}{c}\text { Model X2 } \\
\text { probability }\end{array}$ & Variable & Estimate & Std. error & $z$ value & p-value \\
\hline \multirow{3}{*}{ Spring 2013} & \multirow{3}{*}{$\begin{array}{l}\text { Number } \sim \text { Temp }+\mathrm{BCl}+ \\
\text { Sex }\end{array}$} & \multirow{3}{*}{694.59} & \multirow{3}{*}{$<0.001$} & $\mathrm{BCl}$ & -0.872 & 0.307 & -2.835 & 0.005 \\
\hline & & & & Mean temperature & -2.453 & 0.136 & -18.077 & $<0.001$ \\
\hline & & & & Sex: Male & -0.201 & 0.076 & -2.63 & 0.009 \\
\hline \multirow{5}{*}{ Autumn 2014} & \multirow{5}{*}{$\begin{array}{l}\text { Number } \sim \text { Temp }+\mathrm{RH}+\mathrm{BCl} \\
+\mathrm{Sex}+\mathrm{SVL}\end{array}$} & \multirow{5}{*}{436.99} & \multirow{5}{*}{$<0.001$} & $\mathrm{BCl}$ & 0.24 & 0.137 & 1.755 & 0.079 \\
\hline & & & & Mean RH & -0.01 & 0.005 & -1.817 & 0.069 \\
\hline & & & & Mean temperature & -0.771 & 0.049 & -15.574 & $<0.001$ \\
\hline & & & & Sex: M & 0.379 & 0.059 & 6.388 & $<0.001$ \\
\hline & & & & SVL & -0.011 & 0.001 & -7.878 & $<0.001$ \\
\hline \multirow{4}{*}{ Spring 2014} & \multirow{4}{*}{$\begin{array}{l}\text { Number } \sim \text { Temp }+\mathrm{RH}+\mathrm{BCl} \\
+\mathrm{SVL}\end{array}$} & \multirow{4}{*}{189.39} & \multirow{4}{*}{$<0.001$} & $\mathrm{BCl}$ & 1.108 & 0.329 & 3.368 & 0.001 \\
\hline & & & & Mean RH & 0.064 & 0.006 & 10.242 & $<0.001$ \\
\hline & & & & Mean temperature & -0.157 & 0.089 & -1.762 & 0.078 \\
\hline & & & & SVL & 0.004 & 0.002 & 2.701 & 0.007 \\
\hline \multirow{7}{*}{ Autumn 2015} & \multirow{7}{*}{$\begin{array}{l}\text { Number } \sim \text { Site }+\mathrm{BCl}+\text { Sex } \\
+\mathrm{SVL}\end{array}$} & \multirow{7}{*}{494.37} & \multirow{7}{*}{$<0.001$} & $\mathrm{BCl}$ & 1.713 & 0.181 & 9.464 & $<0.001$ \\
\hline & & & & Sex: Male & -0.296 & 0.077 & -3.711 & $<0.001$ \\
\hline & & & & Site: Cape Sanctuary & 0.102 & 0.084 & 1.208 & 0.227 \\
\hline & & & & Site: Maungatautari & -0.769 & 0.092 & -8.34 & $<0.001$ \\
\hline & & & & $\begin{array}{l}\text { Site: Stephens } \\
\text { Island }\end{array}$ & 0.405 & 0.068 & 5.913 & $<0.001$ \\
\hline & & & & Site: Whangaokeno & -1.747 & 0.32 & -5.469 & $<0.001$ \\
\hline & & & & SVL & 0.011 & 0.309 & 6.517 & $<0.001$ \\
\hline
\end{tabular}


Table A4.2 GLM outputs of best-fit models of predictors influencing tick loads per season per site. Significant predictor variables per site per survey season are shown. Whangaokeno and Young Nicks Head Sanctuary populations were not included as sample sizes were too small to enable reliable statistical analysis $(n \leq 8)$.

\begin{tabular}{|c|c|c|c|c|c|c|c|c|}
\hline Site & Season & $\begin{array}{c}\text { Predictor } \\
\text { variable }\end{array}$ & Model $\chi 2$ & $\begin{array}{c}\text { Model X2 } \\
\text { probability }\end{array}$ & Estimate & Std.Error & $z$ value & p-value \\
\hline \multirow{4}{*}{ Cape Sanctuary } & Spring 2013 & Sex & 6.844 & 0.009 & -1.047 & 0.422 & -2.485 & 0.013 \\
\hline & Autumn 2014 & $\mathrm{BCl}$ & 23.753 & $<0.001$ & 2.700 & 0.580 & 4.658 & $<0.001$ \\
\hline & Spring 2014 & Sex & 5.816 & 0.016 & 0.419 & 0.175 & 2.395 & 0.017 \\
\hline & Autumn 2015 & Sex & 32.017 & $<0.001$ & -0.678 & 0.126 & -5.400 & $<0.001$ \\
\hline \multirow{4}{*}{ Maungatautari } & Spring 2013 & Sex & 6.660 & 0.010 & 1.286 & 0.600 & 2.145 & 0.032 \\
\hline & Autumn 2014 & SVL & 108.230 & $<0.001$ & 0.040 & 0.004 & 9.166 & $<0.001$ \\
\hline & Spring 2014 & $\mathrm{BCl}$ & 14.031 & $<0.001$ & 1.492 & 0.403 & 3.704 & $<0.001$ \\
\hline & Autumn 2015 & Sex & 12.600 & $<0.001$ & -0.528 & 0.148 & -3.572 & $<0.001$ \\
\hline \multirow{6}{*}{ ZEALANDIA } & Spring 2013 & $\mathrm{BCl}$ & 16.392 & $<0.001$ & -1.289 & 0.315 & -4.086 & $<0.001$ \\
\hline & Autumn 2014 & $\mathrm{BCl}$ & \multirow{2}{*}{16.345} & \multirow{2}{*}{$<0.001$} & -0.826 & 0.337 & -2.449 & 0.014 \\
\hline & AucumIII 2014 & Sex & & & -0.210 & 0.091 & -2.319 & 0.020 \\
\hline & Spring 2014 & SVL & 17.449 & $<0.001$ & 0.010 & 0.003 & 4.080 & $<0.001$ \\
\hline & \multirow{2}{*}{ Autumn 2015} & $\mathrm{BCl}$ & \multirow{2}{*}{62.907} & \multirow{2}{*}{$<0.001$} & 1.505 & 0.364 & 4.138 & $<0.001$ \\
\hline & & Sex & & & 0.477 & 0.088 & 5.436 & $<0.001$ \\
\hline \multirow{5}{*}{ Stephens Island } & \multirow{3}{*}{ Autumn 2014} & Sex & \multirow{3}{*}{166.53} & \multirow{3}{*}{$<0.001$} & 0.471 & 0.075 & 6.261 & $<0.001$ \\
\hline & & $\mathrm{BCl}$ & & & 2.529 & 0.261 & 9.705 & $<0.001$ \\
\hline & & SVL & & & -0.012 & 0.002 & -6.037 & $<0.001$ \\
\hline & \multirow{2}{*}{ Autumn 2015} & $\mathrm{BCl}$ & \multirow{2}{*}{85.082} & \multirow{2}{*}{$<0.001$} & 1.865 & 0.232 & 8.050 & $<0.001$ \\
\hline & & SVL & & & 0.005 & 0.001 & 3.339 & 0.001 \\
\hline
\end{tabular}




\section{Appendix 5}

Table A5.1 Air temperature and percentage of emerged tuatara per site at all 1100 observations for the spring 2014 and autumn 2015 surveys.

\begin{tabular}{|c|c|c|c|c|c|c|c|}
\hline Season & Site & $\begin{array}{c}\text { Temp } \\
\left({ }^{\circ} \mathrm{C}\right)\end{array}$ & $\begin{array}{c}\text { Emergence } \\
(\%)\end{array}$ & Season & Site & $\begin{array}{c}\text { Temp } \\
\left({ }^{\circ} \mathrm{C}\right)\end{array}$ & $\begin{array}{c}\text { Emergence } \\
\text { (\%) }\end{array}$ \\
\hline \multirow{28}{*}{$\begin{array}{l}\text { Spring } \\
2014\end{array}$} & \multirow{7}{*}{ Cape Sanctuary } & 14.4 & 80 & \multirow{28}{*}{$\begin{array}{l}\text { Autumn } \\
2015\end{array}$} & \multirow{7}{*}{ Cape Sanctuary } & 16.0 & 33 \\
\hline & & 22.7 & 33 & & & 19.1 & 56 \\
\hline & & 23.2 & 56 & & & 12.4 & 33 \\
\hline & & 23.4 & 20 & & & 11.0 & 33 \\
\hline & & 23.8 & 50 & & & 15.0 & 22 \\
\hline & & 24.0 & 70 & & & 16.0 & 22 \\
\hline & & 25.7 & 44 & & & 19.2 & 33 \\
\hline & \multirow{7}{*}{ Maungatautari } & 11.5 & 50 & & \multirow{7}{*}{ Maungatautari } & 19.7 & 0 \\
\hline & & 13.3 & 17 & & & 17.9 & 0 \\
\hline & & 14.2 & 33 & & & 17.6 & 40 \\
\hline & & 14.7 & 33 & & & 17.2 & 25 \\
\hline & & 15.9 & 67 & & & 18.8 & 25 \\
\hline & & 17.0 & 17 & & & 18.6 & 20 \\
\hline & & 18.8 & 25 & & & 15.6 & 40 \\
\hline & \multirow{7}{*}{ ZEALANDIA } & 11.6 & 40 & & \multirow{7}{*}{ Stephens Island } & 14.9 & 40 \\
\hline & & 12.2 & 17 & & & 14.1 & 20 \\
\hline & & 12.6 & 40 & & & 19.0 & 50 \\
\hline & & 13.9 & 25 & & & 19.1 & 70 \\
\hline & & 13.9 & 60 & & & 16.3 & 44 \\
\hline & & 14.3 & 40 & & & 17.1 & 13 \\
\hline & & 15.7 & 50 & & & 15.0 & 38 \\
\hline & & & & & \multirow{7}{*}{ ZEALANDIA } & 13.3 & 29 \\
\hline & & & & & & 15.7 & 50 \\
\hline & & & & & & 15.5 & 38 \\
\hline & & & & & & 16.5 & 67 \\
\hline & & & & & & 14.9 & 11 \\
\hline & & & & & & 15.8 & 38 \\
\hline & & & & & & 9.3 & 0 \\
\hline
\end{tabular}

